\title{
Interpretation of Geological Correlation Borings 1, 2, 3 in the A/M Area of the Savannah River Site, South Carolina
}

by

D. E. Wyatt

Westinghouse Savannah River Company

Savannah River Site

Aiken, South Carolina 29808

R. J. Cumbest

R. K. Aadland

F. H. Syms

D. E. Stephenson

J. C. Sherrill

This paper was prepared in connection with work done under the above contract number with the U.S. Department of Energy. By acceptance of this paper, the publisher and/or recipient acknowledges the U.S. Government's right to retain a nonexclusive, royalty-free license in and to any copyright covering this paper, along with the right to reproduce and to authorize others to reproduce all or part of the copyrighted paper.
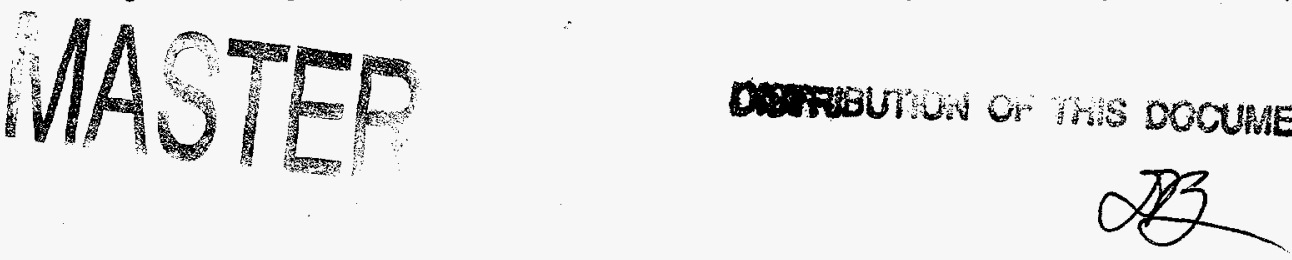


\section{DISCLAIMER}

This report was prepared as an account of work sponsored by an agency of the United States Government. Neither the United States Government nor any agency thereof, nor any of their employees, makes any warranty, express or implied, or assumes any legal liability or responsibility for the accuracy, completeness, or usefulness of any information, apparatus, product, or process disclosed, or represents that its use would not infringe privately owned rights. Reference herein to any specific commercial product, process, or service by trade name, trademark, manufacturer, or otherwise does not necessarily constitute or imply its endorsement, recommendation, or favoring by the United States Government or any agency thereof. The views and opinions of authors expressed herein do not necessarily state or reflect those of the United States Government or any agency thereof.

This report has been reproduced directly from the best available copy.

Available to DOE and DOE contractors from the Office of Scientific and Technical Information, P. O. Box 62, Oak Ridge, TN 37831; prices available from (423) 576-8401.

Available to the public from the National Technical Information Service, U. S. Department of Commerce, 5285 Port Royal Road, Springfield, VA 22161. 


\section{DISCLAIMER}

Portions of this document may be illegible electronic image products. Images are produced from the best available original document. 
Upper Three Runs Watershed

A/M Area Advanced Geological Study

Part 2 of 3

Interpretation of Geological Correlation

Borings 1, 2, 3 in the A/M Area of the Savannah River Site, South Carolina (U)

D. E. Wyatt

R. J. Cumbest

R. K. Aadland

F. H. Syms

D. E. Stephenson

J. C. Sherrill

Site Geotechnical Services

Westinghouse Savannah River Company

P. O. Box 616

Aiken, South Carolina 29808

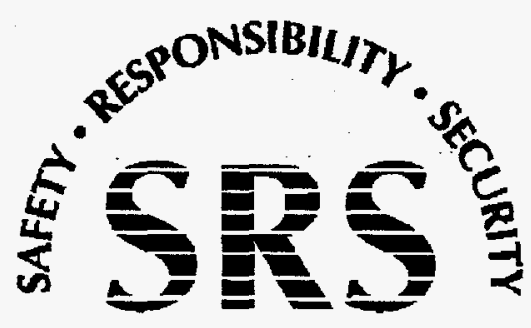

SAVANNÄH RIVER SITE 
Upper Three Runs Watershed A/M Area Advanced Geological Study Part 1 of 3

Interpretation of Geological Correlation Borings 1, 2, 3 in the A/M Area of the Savannah River Site, South Carolina (U)

D. E. Wyatt

R. J. Cumbest

R. K. Aadland

F. H. Syms

D. E. Stephenson

J. C. Sherrill

Site Geotechinicäl Services

Westinghouse Savannah River Company

P. O. Box 616

Aiken, South Carolina 29808 
WSRC-RP-97-0185

Revision 0

June, 1997

The A/M Area Advanced Geological Study is comprised of three separate reports, Parts 1 through 3. The parts are:

Part 1

Investigation on the Combined Use of Ground Penetrating Radar, Cone Penetrometer and High Resolution Seismic Data for Near Surface and Vadose Zone Characterization in the AMM Area of the Savannah River Site, South Carolina (U)

\section{Part 2}

Interpretation of Geological Correlation Borings 1, 2, 3 in the AM Area of the Savannah River Site, South Carolina (U)

Part 3

Geological Interpretation of the Structure and Stratigraphy of the AM Area, Savannah River Site, South Carolina (U)

This report was prepared by Westinghouse Savannah River Company (WSRC) for the United States Department of Energy under Contract No. DE-AC09-96SR18500. and is an account of work performed under that contract. Reference herein to any specific commercial product, process, or services by trademark, name, manufacturer or otherwise does not necessarily constitute or imply endorsement, recommendation, or favoring of same by WSRC or by the United States Government or any agency thereof. The views and opinions of the authors expressed herein do not necessarily state or reflect those of the United States Government or any agency thereof. 


\section{TABLE OF CONTENTS}

Executive Summary 3

Introduction 4

$\begin{array}{ll}\text { Field Activities } & 6\end{array}$

$\begin{array}{ll}\text { Interpretation } & 7\end{array}$

Hydrogeologic Relationships $\quad 22$

$\begin{array}{ll}\text { Summary } & 26\end{array}$

$\begin{array}{ll}\text { References } & 28\end{array}$

Appendices

Appendix A - USGS Field Core Descriptions for GCB-1, 2, 3

Appendix B - Suspension Velocity Measurements for GCB-1 and 2

Appendix C - Palynology Correspondence for GCB-1 and 2

Appendix D - Core Plug VOC Data for GCB-1, 2, 3

List of Figures

Figure 1. Location of GCB-1, 2, 3

Figure 2. Stratigraphic Correlation Panel

Figure 3. GCB-1 Geophysical and Core Interpretation 10

Figure 4. GCB-2 Geophysical and Core Interpretation 11

Figure 5. GCB-3 Geophysical and Core Interpretation 12

Figure 6. Sand - Mud - Gravel Ternary Diagram 23

Figure 7. Z-plot of Confining Units for GCB-1 24

Figure 8. Z-plot of Confining Units for GCB-3 25

List of Tables

Table 1. Geophysical Logs for GCB-1,2,3 
This page intentionally left blank. 


\section{EXECUTIVE SUMMARY}

The Geophysical Correlation Boring (GCB) Program was organized to provide a comprehensive correlation capability between geological core and advanced borehole geophysical data, surface high resolution reflection seismic information and, when available, borehole geochemical and cone penetrometer data. This report provides results and initial geological interpretations of borings one, two and three (GCB-1, GCB-2, GCB-3) located within the Upper Three Runs Watershed (AMM Area) of the Savannah River Site.

The interpretation of the GCB borings is guided by the need to establish relationships between core and geophysically derived data. Additionally, the correlation between the geophysical log interpretation and surface derived seismic data is important to understanding the overall A/M, and SRS, geological setting. The GCB-1,2,3 interpretation is presented in a unit by unit correlation comparing core geology and geophysical log derived depositional signatures.

The interpretation of the lithostratigraphy of GCB-1,2,3 suggests that GCB-3 contains sediments that were generally deposited in a more near-shore, subtidal to fluvial, updip environment than sediments in GCB-1 or 2. GCB-1 contains lithologies that are deeper water, subtidal to deltaic while GCB-2 has similar sediments that were deposited in a slightly shallower environment. The present relationship between GCB-1,2,3 indicates that GCB-2 is structurally lower relative to GCB-1 and 3. Correlation of strata between the borings suggests that basement structural events continued through the Paleocene and are terminated by the Lang Syne/Sawdust Landing unconformity. Thickening of sediments within the structurally low depression of GCB-2 after the Lang Syne/Sawdust Landing unconformity suggests a structural reactivation during the Eocene.

In general, GCB-1 and 2 contain intervals with sufficient amounts of clay or fine grained silty material to provide localized confining units throughout the stratigraphic column. GCB-3 contains only minimal clay amounts in thin intervals with minimal confining capability in the Paleocene and Eocene, while thicker clays, with large amounts of silt, are present in the Cretaceous.

The analysis of laboratory core, field core and geophysical log derived data in the GCB borings provides a powerful tool for a comprehensive geological analysis of the subsurface of the Upper Three Runs Watershed. Environments of deposition, structural and stratigraphic correlation and aquifer characteristics may be derived from a combination of these data, leading to a more powerful predictive tool for groundwater flow in support of remedial efforts. 


\section{INTRODUCTION}

The Geophysical Correlation Boring (GCB) Program was organized to provide a comprehensive correlation capability between geological core and advanced borehole geophysical data, surface high resolution reflection seismic information and, when available, borehole geochemical and cone penetrometer data. Borings were planned in strategic locations within the Upper Three Runs Watershed (AM Area). All boring locations were chosen to maximize correlation to existing or newly acquired borehole and seismic data and to compliment existing or ongoing environmental programs. This report provides results and initial interpretations of borings one, two and three (GCB-1, GCB-2, GCB-3) located within the A/M Area (Figure 1).

The Geophysical Correlation Boring Program was a joint effort supported by several organizations and agencies. Funding for the program was provided through the WSRC Environmental Restoration Department and administered by WSRC Site Geotechnical Services. Drilling and field oversight were provided by the US Geological Survey through an inter-agency agreement administered through the DOE-SRS Environmental Division. All field work was coordinated through WSRC Site Geotechnical Services and the USGS provided field core descriptions. Additional subcontract services were provided as follows: drilling by EM\&TC, Inc., binocular core descriptions by Science Applications International Corporation, field support and coordination by Bechtel Savannah River, Inc., and the Raytheon Corporation. Core geochemical sampling was provided by Microseeps, Inc. Core from the upper 150 feet $(50 \mathrm{~m})$ of each boring was split and sent to Rutgers University for detailed soils and transport analysis as part of the DOE-HQ Consortium for Risk Evaluation with Stakeholder Participation (CRESP) program. Borehole geophysical data were acquired by Schlumberger Wireline Services, Inc., and Western-Atlas Wireline, Inc. Additional well logging was provided by Graves Logging Services. Downhole acoustic suspension logging was acquired in GCB-1 and GCB-2 by Agbabian and Associates. Surface seismic information was obtained and processed by the Earth Sciences and Resources Institute of the University of South Carolina. Palynology data and additional core analysis was provided by the Department of Geological Science of Clemson University. Ground Penetrating Radar data for the GCB program was acquired and processed by Microseeps, Inc. All data analysis, interpretation and correlation was provided by WSRC, Site Geotechnical Services.

From this data, lithostratigraphic and hydrostratigraphic interpretations and relationships may be evaluated. This report provides a description of environments of deposition, sedimentary relationships and hydrostratigraphic character for the sediments associated with borings GCB-1, 2 and 3 as correlated on Figure 2. 


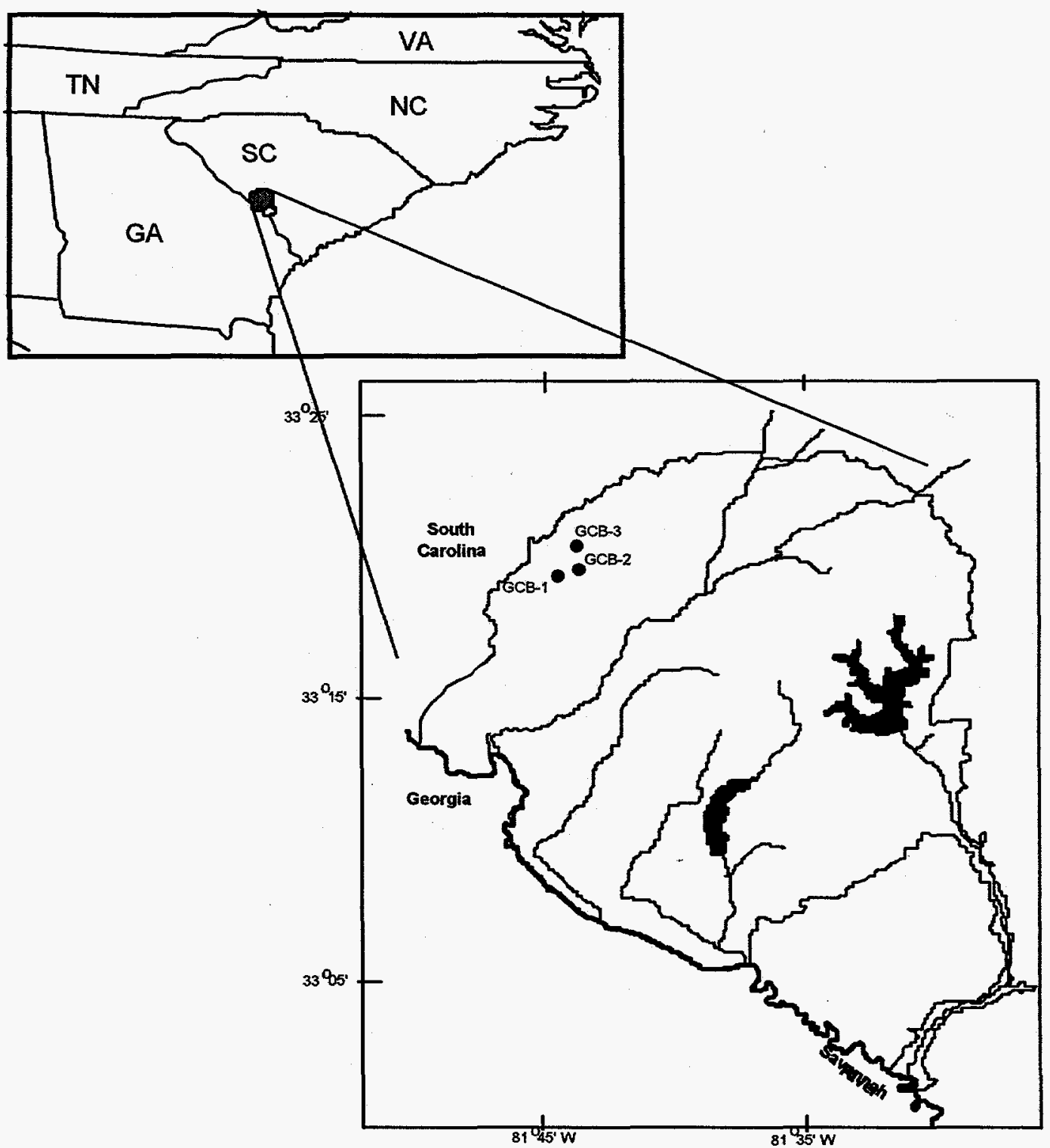

Figure 1. Location of the Geophysical Correlation Borings, GCB-1, 2, 3 within the Savannah River Site on the South Carolina upper coastal plain. 


\section{FIELD ACTIVITIES}

The following field activities are described individually by boring.

\section{GCB-1}

The GCB-1 boring was spudded, as GCB-1A, on July 19, 1996 by EM\&TC. Coring resumed on July 22 and continued through July 28 to a depth of $327 \mathrm{ft}$ (99.6 m). Western Atlas acquired geophysical logs to a depth of 327 feet (99.6 $\mathrm{m})$. Coring resumed on August 1 and continued to August 7, terminating at 500 feet $(152 \mathrm{~m})$. Graves Wireline acquired geophysical logs on the upper 500 feet. No casing was required in this boring. Coring resumed on August 25 and continued until September 3 to a depth of 802.7 feet $(244.6 \mathrm{~m}$ ). Schlumberger Wireline Services acquired a full suite of geophysical logs to the total depth of the boring. Agbabian and Associates obtained a suspension log to the total depth of the boring. Core was recovered and described in the field by USGSWater Resources Division Geologist Kevin Conlan. Drilling fluid halogenated gas samples were acquired periodically through the upper 150 feet $(45 \mathrm{~m})$ to evaluate whether Investigation Derived Wastes (IDW) concerns were required. Soil geochemical plugs were obtained approximately every five to 10 feet, if the interval was a sand zone, and analyzed for halogenated solvents. These data are reported in Appendix D. Selected intervals of the remaining core were split and provided to Clemson University for a detailed stratigraphic and palynological analysis. Geophysical logs obtained are listed in Table 1. A detailed foot by foot core analysis was completed by SAIC and is shown in the lithology column of Figure 3. Detailed field descriptions are reported in.Appendix A.

\section{GCB-2}

The GCB-2 boring was spudded on June 4, 1996 and cored continuously through June 11 to a depth of 492 feet $(150 \mathrm{~m})$. Casing was set in the upper 140 feet $(42.6 \mathrm{~m})$ because of soft hole conditions and lost circulation. Coring resumed on June 20 and drilled continuously, except for bit and shoe problems, until June 29 at a total depth of 684 feet $(208.5 \mathrm{~m})$. Core was recovered and described in the field by USGS-Water Resources Division Geologist Kevin Conlan. Drilling fluid halogenated gas samples were acquired periodically through the upper 150 feet $(45.7 \mathrm{~m})$ to evaluate whether IDW concerns were required. Soil geochemical plugs were obtained approximately every five to 10 feet $(1.5$ to $3 \mathrm{~m})$, if the interval was a sand zone, and analyzed for halogenated solvents. These data are reported in Appendix D. The remaining core was split and provided to Clemson University for a detailed stratigraphic and palynological analysis. The boring was geophysically logged on July 2, 1996 by Schlumberger Wireline Services. Geophysical logs obtained are listed in Table 1. A detailed foot by foot core analysis was completed by SAIC and is shown in the lithology column of Figure 4. Detailed field descriptions are reported in Appendix A. 


\section{GCB-3}

The GCB-3 boring was spudded on July 1, 1996 and cored continuously through July 2 to a depth of 213 feet $(65 \mathrm{~m})$. No casing was required in this boring. Coring resumed on July 11 and continued, except for bit and shoe problems, until July 16 to a depth of 430 feet $(131 \mathrm{~m})$, when an injury occurred to a member of the drill crew requiring a stand down until July 20 . Coring resumed on July 20 and continued until July 22 to a total depth of 632 feet $(192.6 \mathrm{~m})$. At this depth, Western Atlas acquired geophysical logs. Core was recovered and described in the field by USGS-Water Resources Division Geologist Kevin Conlan. Drilling fluid halogenated gas samples were acquired periodically through the upper 150 feet $(45.7 \mathrm{~m})$ to evaluate whether IDW concerns were required. Soil geochemical plugs were obtained approximately every five to 10 feet $(1.5$ to $3 \mathrm{~m})$, if the interval was a sand zone, and analyzed for halogenated solvents. These data are reported in Appendix D. After review, it was decided that the total depth of the boring was not sufficient for acoustical logging to support seismic interpretation and the hole was deepened to 652 feet $(198.7 \mathrm{~m})$. Schlumberger Wireline Services acquired sonic and spectral data for the total depth of the boring. Selected intervals of the remaining core were split and provided to Clemson University for a detailed stratigraphic and palynological analysis. Geophysical logs obtained are listed in Table 1. A detailed foot by foot core analysis was completed by SAIC and is shown in the lithology column of Figure 5. Detailed field descriptions are reported in Appendix A.

GCB-1

Digital Sonic

Formation Micro-Imager

Natural Gamma Spect.

Density/Porosity

Neutron/Porosity

Array Induction

Mag. Resonance

Caliper
GCB-2

Digital Sonic

Natural Gamma Spect.

Density/Porosity

Neutron/Porosity

Dual Induction Focused

Caliper
GCB-3

Digital Sonic

Natural Gamma Spect.

Density/Porosity

Neutron/Porosity

Dual Induction Focused

Caliper

Mag. Resonance

Table 1. Geophysical logs obtained from the GCB borings. The utility of each log is discussed in the Summary section.

\section{INTERPRETATION}

The interpretation of the GCB borings is guided by the need to establish relationships between core derived and geophysically derived geology. Additionally, the correlation between the geophysical log interpretation and 
surface derived seismic data is important to understanding the overall. $A M$, and SRS, geological setting. The GCB-1,2,3 interpretation will be presented as a unit by unit correlation comparing core geology and log derived depositional signatures. The stratigraphic unit name is followed by the referenced horizon name for Figures 2 through 5 . Hydrostratigraphic nomenclature follows that of Aadland et al. (1995). Stratigraphic nomenclature and environmental comparisons are from Fallaw and Price (1995) and Reineck and Singh (1980). Log interpretations follow suggestions from Schlumberger (1989) and Serra (1985). Table 2 compares basic geophysically derived physical parameters for the units.

\section{Saprolite and Basement (Pzm/Sap and Acoustic Basement)}

Crystalline basement lithologies are similar across the GCB-1,2,3 area. In GCB1 , an olive to gray/green weathered chlorite schist overlies a highly fractured bluish gray gneiss. The thickness of the schist is approximately 47 feet $(14.3 \mathrm{~m})$ and a distinct contact is present between the schist and gneiss. In GCB-2 and 3, only the chlorite schist interval was sampled which had a similar appearance to the schist of GCB-1. Fractures within the schist of GCB-1 have an approximate northwest strike orientation with a dip varying between 13 and 27 degrees. Fractures and quartz veins within the gneiss appear to have a northeast strike orientation and gentle dips, however, only the upper few feet of the gneiss were sampled for dip information. No oriented dip or strike information is available for GCB-2 and 3. All of the crystalline basement lithologies encountered in GCB-1,2 and 3 were expected as referenced in Cumbest and Price (1989).

The top of basement contact is the first contact with the highly weathered and fractured saprolite. Generally, the saprolite increases in thickness from GCB-2 and 3 to GCB-1 from approximately 13 feet $(4 \mathrm{~m})$ in GCB-3 and 15 feet in GCB-2 to approximately 32 feet $(9.8 \mathrm{~m})$ in GCB-1. The character of the saprolite varies in each boring. In GCB-1 the saprolite contact is defined by a sharp change between a well indurated sandy/silty clay to gray-green clay with numerous iron oxide stains. The color varies from gray-green to brown-red. In GCB-2 the saprolite is a brown to red mottled clay grading into a dense, massively foliated olive green clay while in GCB-3 the saprolite is a bluish gray to off white, well indurated clay.

\section{Cape Fear Formation (Top CF)}

The Cape Fear Formation generally thickens towards the southeast from GCB-3 to GCB-1. The overall lithology of the formation consists of interbedded gravel, sands and clays of varying colors and sizes. Rock fragments and angular sands suggest that the Cape Fear Formation was deposited near its source. There are generally three member sequences, alternating coarsening and fining upwards, that define the formation and that are most developed in GCB-1. The overall 
GCB-1

GCB-2

GCB-3

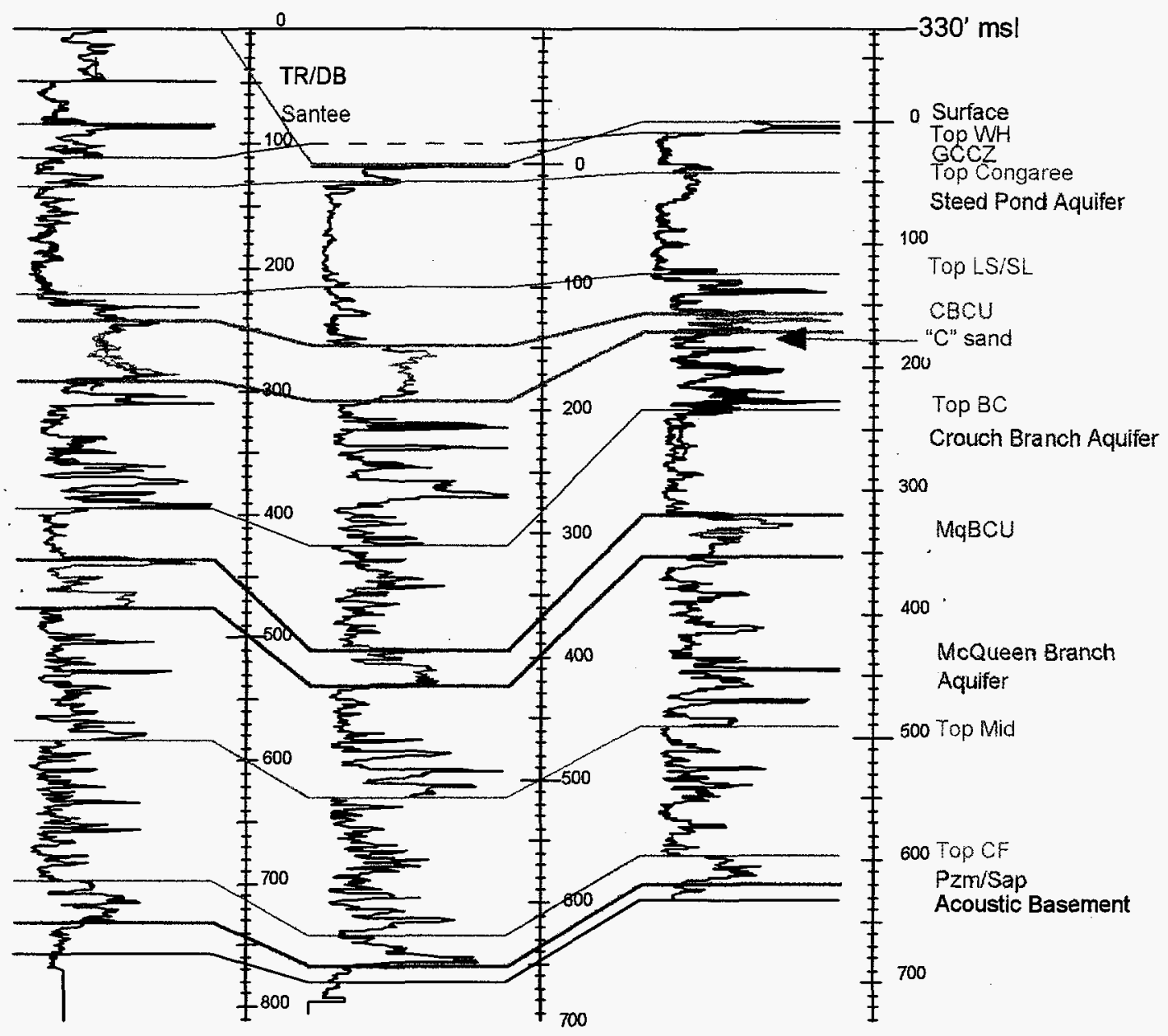

Figure 2. GCB-1, 2 and 3 correlation panel. Datum is set to $330^{\prime}(110 \mathrm{~m}) \mathrm{msl}$. Depths are in feet. Color of correlation lines correspond to color of horizon name. "Top WH" is the top of the Warley Hill Formation. "GCCZ" is the Green Clay Confining Zone. "Top LS/SL" if the top of the Lang Syne/Sawdust Landing Formations. "CBCU" is the Crouch Branch Confining Unit. "Top BC" is the top of the Black Creek Formation. "MqBCU" is the McQueen Branch Confining Unit. "Top Mid" is the top of the Middendorf Formation. "Top CF" is the top of the Cape Fear Formation. "Pzm/Sap" is the contact with the weathered basement rocks or saprolite. Acoustic Basement is the velocity crossover from coastal plain seismic velocities to those more typical of crystalline rocks. 


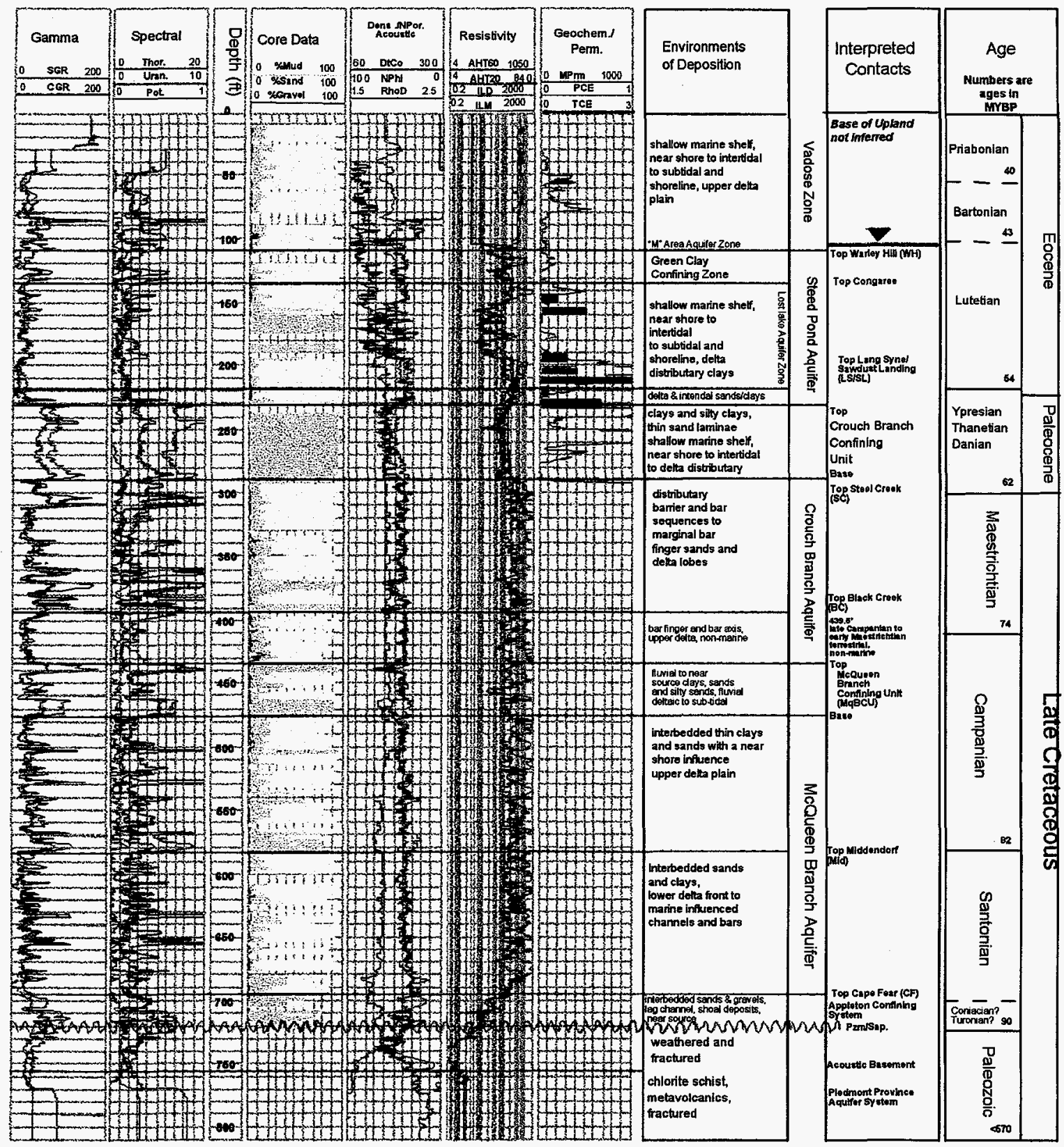

GCB-1

Figure 3. Combined interpretation of GCB-1. Principal geophysical curves are shown. Stratigraphic picks are from the correlation of GCB-1,2 and 3 to regional horizons. Depositional environments are interpreted from the geophysical log signatures, field core descriptions and core laboratory data and are relevant for this figure and Figures 3 and 4. Hydrostratigraphy is from Aadland et al., (1995). Stratigraphy and chronostratigraphy are from Aadland et al. (1995), Fallaw and Price, (1995) and DNAG, 1983. 


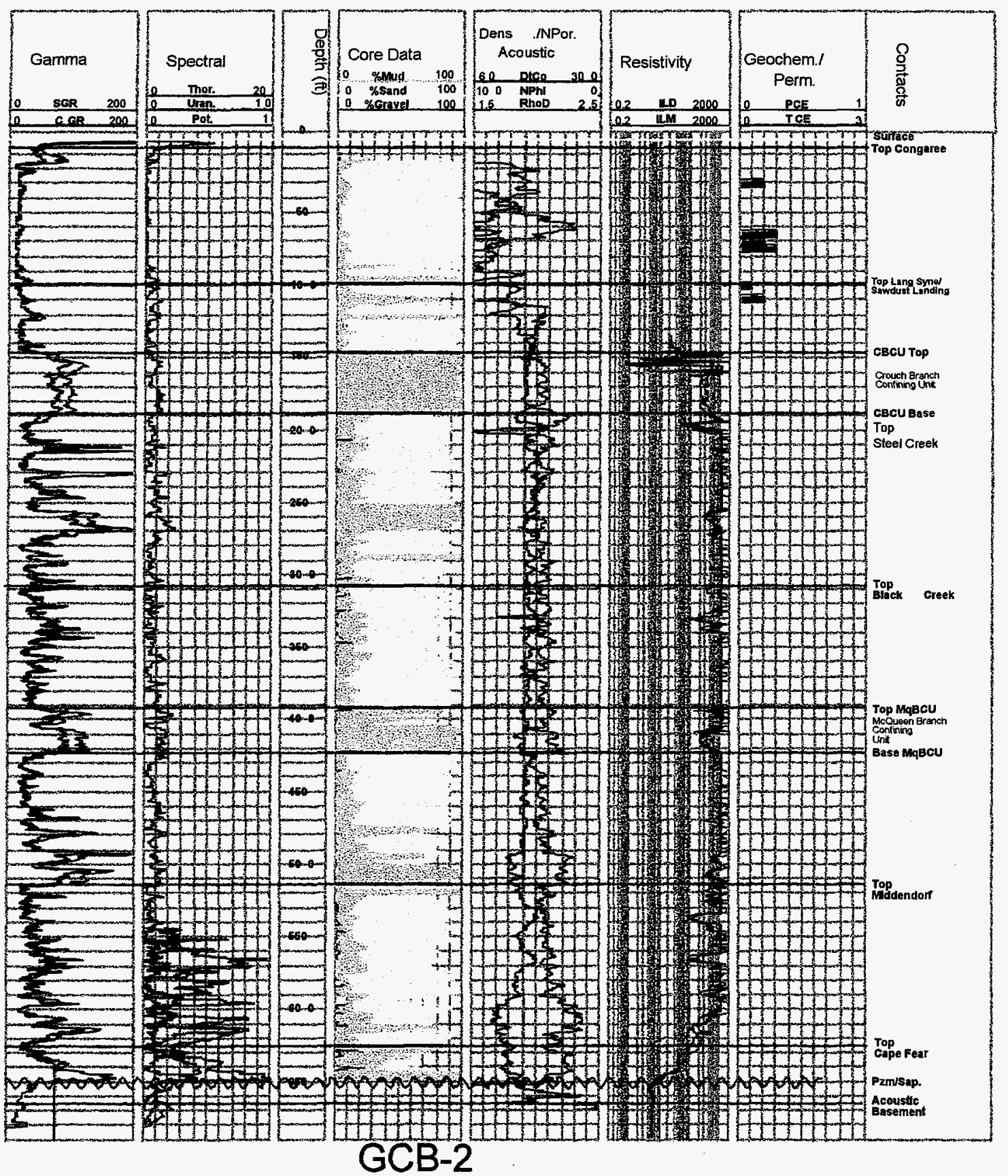

Figure 4. Stratigraphic correlation plot for GCB-2. Refer to Figure 3 for corresponding hydrostratigraphy, chronostratigraphy and depositional environments. 


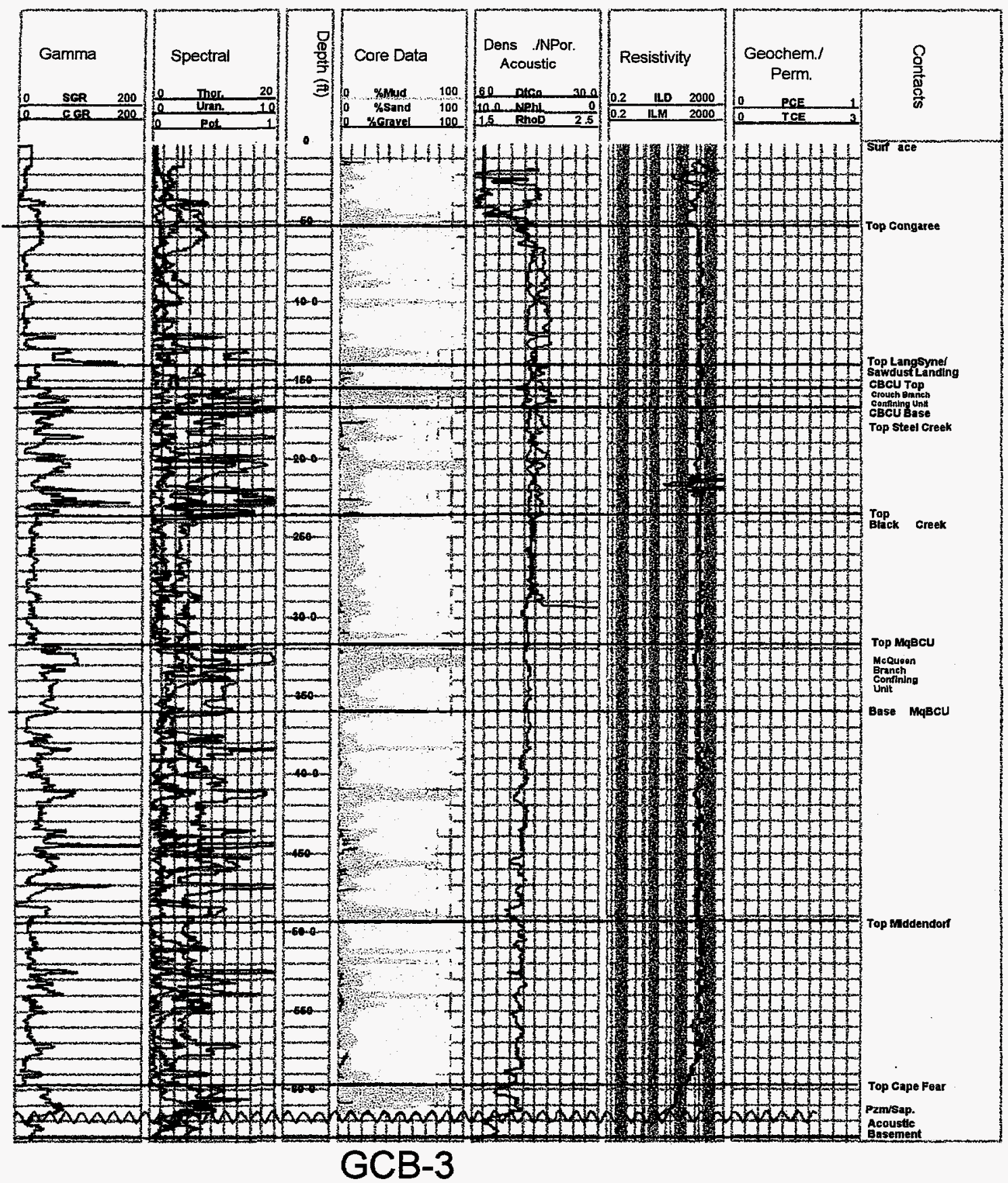

Figure 5. Stratigraphic correlation plot for GCB-3. Refer to Figure 3 for corresponding hydrostratigraphy, chronostratigraphy and depositional environments. 
geophysical log pattern of the sequences, which matches the field core descriptions, suggests that each unit within the formation is a lag channel, shoaling deposit fill with pebbles and heavy sands grading into finer sands with interspersed clays. This interpretation is consistent with the description in Aadland et al., (1995) and Fallaw and Price, (1995).

Within the Cape Fear and at the basal Middendorf, rock resistivity values and deep versus shallow curve separation (an indicator of relative permeability) decrease rapidly. The overall neutron porosity drops from an average of approximately $35 \%$ to approximately $20 \%$. The low relative permeability and porosity suggests tighter formations. The lower resistivity values are associated with higher concentrations of heavy minerals and interstitial waters are more mineralized than shallower aquifers. Overall, the Cape Fear and saprolite are indurated with lower permeability, forming the Appleton Confining Unit.

\section{Middendorf Formation (Top Mid)}

The Middendorf Formation gradually thickens towards the southeast from GCB-3 to GCB-1. There are correlatable packages of sediments across the formation but it is difficult to correlate specific sand or clay units across the area defined by GCB-1, 2 and 3. Channel and sheet type sands are interbedded with indurated clays suggesting a lower delta plain depositional environment. Many of the sand units in GCB-1,2,3 appear to alternate between fining and coarsening upwards suggesting that they are marine influenced channels or bars. Spectral gamma data, when combined with field core descriptions and lab core analysis, suggests that there are few clays of significant thickness within the formation. Generally, the clays are thin and less than one meter thick. Numerous heavy minerals and micas, as well as fine grained sands with clay balls, generate a spiked appearance of the gamma curve which may be interpreted as numerous interspersed clays. Focused or Induction Resistivity data suggests that there are numerous high permeability zones separated by numerous 'tight' streaks of clay or silt to silty-sand. These zones also suggest that there are non-correlatable sand bodies within the formation with variable permeability, therefore, tracking groundwater movement within the Middendorf within the AMM Area would be extremely difficult.

\section{Black Creek Formation (Top BC)}

The Black Creek Formation in borings GCB-1,2,3 may be described in terms of an upper and lower member, possibly two sequences, separated by the McQueen Branch Confining Unit (MqBCU) regional aquitard. Both upper and lower zones have geophysical log and core characteristics that define them as primary regional aquifers. 
The lower zone lies unconformably over the Middendorf Formation and consists of interbedded thin clays and sands occurring, more or less, randomly distributed and uniformly spaced throughout the interval. Spectral gamma peak to peak correlation, indicating regional clay units, are possible to track across the borings. These clays are probably localized maximum flooding surfaces, while the basal clays, similar across the area, were deposited in a near shore marine environment. The interbedded sands and clays, often with interspersed iron staining and heavy minerals, is suggestive of a fluvial to upper delta depositional environment.

The MqBCU is a sequence of three principal clays and silty clays occurring in a 40 foot $(12.2 \mathrm{~m})$ interval separated by clayey to silty sands. Each of the three units is approximately 10 to 15 feet $(3.0$ to $4.6 \mathrm{~m}$ ) thick with the lower unit having the most consistent clay content and thickness. Many of the sands within the $\mathrm{MqBCU}$ are moderately sorted and sub-angular with a consistent presence of micas suggesting that these sands are fluvial and deposited near their source. The clays also consistently have micas presence, suggesting that the clays and the sands are both fluvial deltaic to near shore sub-tidal.

The upper zone of the Black Creek is variable across the region of the GCB borings. In GCB-2, the interval consists principally of interbedded clays and sands in beds averaging 5 to 7 feet $(1.7$ to $2.1 \mathrm{~m})$ thick. This 'serrated' depositional style is common along the margins of bar-finger sands (Busch and Link, 1985). In GCB-1 and 3, the dominant lithology in the upper zone is sand with thin interspersed silt and clay intervals. The sands are dominantly moderately to well sorted, tan to cream in color, and sub-angular to subrounded. Micas are present and sometimes abundant with rare heavy minerals. The sand is 75 feet $(22.9 \mathrm{~m})$ thick in GCB-3 and 37 feet $(11.4 \mathrm{~m})$ thick in GCB-1. These sands are not massive but consist of stacked bar finger, bar-axis and barrier sequences with dips (in GCB-1) of $2^{\circ}$ to $12^{\circ}$ to the northwest in the upper units, to $14^{\circ}$ to $21^{\circ}$ to the south-southwest in the middle sands followed by $15^{\circ}$ to $29^{\circ}$ dips to the northeast in the lower sand units. The average dip direction is to the south. The alternating dip directions are indicative of near shore influence and the measured dips are probably alternating barrier or dune foreset beds.

Samples for palynological analysis were obtained from the GCB-1 boring in a dark, organic rich clay from a depth of 439.5 feet $(134 \mathrm{~m})$ at the base of the upper sand unit and top of the MqBCU. Palynomorphs were present indicating a late Campanian to early Maestrichtian age. The spores, pollen, plant cuticle, wood fibers and inertinite, plus a lack of marine algae, indicates that this interval is non-marine (Christopher, personnel communication, 1996). 
Steel Creek Formation (Base of CBCU to Top BC)

The Steel Creek Formation is defined from the base of the Crouch Branch Confining Unit (CBCU) to the top of the Black Creek Formation. Dominant lithologies consist of micaceous clays interbedded with sands, sands and silty sands interbedded with thin clays and pebble zones. Micas, including angular grains, are present throughout the interval and iron staining is common. In the basal 50 feet $(15.2 \mathrm{~m})$ of each boring, clay content increases and alternates with fine grained silty sands. The overall dip direction is generally random. Sands appear to be more prevalent in the upper portions of the Steel Creek and exhibit morphologies similar to distributary barrier and bar sequences. In GCB-1, these sands generally fine upwards, and exhibit varying dip angles from up to $20^{\circ}$ northwest to $12^{\circ}$ southeast and $18^{\circ}$ northeast. The ' $\mathrm{C}^{n}$ sand of Aadland et al., (1995) is present in all wells and gradually varies from a coarsening upwards distributary bar finger and delta lobe sands (Busch and Link, 1985) in GCB-1 and 2 to a more marginal bar finger sand or delta lobe in GCB-3. The remaining sands in the upper portion of the Steel Creek are depositionally similar to the ' $C$ ' sand but tend to be more tidal in GCB-3. In general, the sands of the Steel Creek appear to be more landward in GCB-3 and more seaward in GCB-1. It is possible that the Cretaceous/Tertiary boundary $(\mathrm{K} / \mathrm{T})$, and contact with the Paleocene, is present at the basal contact of the clay unit immediately overlying the ' $\mathrm{C}$ ' sand. The presence of pebble lag layers and heavy minerals may define this time sequence marker.

\section{Crouch Branch Confining Unit (Top CBCU to Base CBCU)}

The Crouch Branch Confining Unit varies in thickness and clay content from approximately 17 feet $(5.2 \mathrm{~m}$ ) of thin and sparse clays in GCB-3 to 44 feet (13.4 $\mathrm{m}$ ) of thick silts and clays in GCB-2 to 50 feet $(15.2 \mathrm{~m})$ of silty clays and clays in GCB-1. The clays tend to be a white to light gray, plastic kaolinite with thinly laminated purple mineral horizons. The CBCU in GCB-1 is predominantly kaolinitic clay with only minor interspersed laminae of silty clay. In GCB-2, thin lamina of heavy minerals and silts are interspersed within the clays. Thin sands within the unit often have heavy minerals and abundant micas. Thin zones of cemented iron laminae are also present. In GCB-3, only thin clay units are present interspersed with silty clays, sandy silts and sands. Numerous lamina with iron staining and heavy minerals are present. The thin sands tend to grade into pebbles and clay balls. The thin clays, clay balls and shoaling sands units in GCB-3 suggest that GCB-3 CBCU sediments were deposited in a fairly high energy, near shore, possibly intertidal zone, transitioning into a subtidal to delta distributary depositional environment in GCB-1.

This unit, which consists of parts of various formations, forms the principal confining unit across the SRS; therefore is discussed in detail as it controls downward contaminant movement. Permeability variations in the $\mathrm{CBCU}$, 
determined from magnetic resonance geophysical logs, are observable in GCB1. The interval from 240 feet $(73.2 \mathrm{~m})$ to 250 feet $(76.2 \mathrm{~m})$ is a well indurated and mottled clay. Permeability average less than 200 millidarcies. From 240 feet $(73.2 \mathrm{~m})$ to 265 feet $(80.8 \mathrm{~m})$, subsurface, the measured permeabilites increase to greater than 1 darcy. All clay within this interval is well indurated and without other noticeable features. Various color changes, suggesting changes in the geochemical makeup of the clays, form numerous lamina throughout the section. The magnetic resonance tool is probably imaging the horizontal permeability associated with these lamina. In the base of the CBCU, from approximately 265 feet $(80.8 \mathrm{~m})$ to the top of the Steel Creek Formation at 290 feet $(88.4 \mathrm{~m})$ subsurface, the permeability decreases from approximately 500 millidarcies to zero. This interval is also a well indurated clay with fewer lamina, grading into a silty, micaceous clay. At 290 feet $(88.4 \mathrm{~m})$ subsurface, the magnetic resonance permeability increase dramatically in the sands of the Steel Creek Formation.

\section{Lang Syne/Sawdust Landing Members (Top LS/SL to Top CBCU)}

The Lang Syne and Sawdust Landing Formations (refer to Aadland et al., 1995 and Fallaw and Price, 1995 for nomenclature discussions) are not lithologically differentiated within the AMM Area of the Savannah River Site. Within the area of GCB-1,2,3, these formations vary in thickness between 28 feet $(8.5 \mathrm{~m})$ in GCB-3 to 46 feet $(14 \mathrm{~m})$ in GCB-2 and 12 feet $(3.6 \mathrm{~m})$ in GCB-1. Two distinct, lithologically different and correlatable, members are seen in each boring. The basal member of the Lang Syne/Sawdust landing is a lower bar to delta sheet sand that thins downdip to the southeast. The upper member is a silty clay to clay coarsening upwards into a delta front sheet sand that has the greatest thickness in GCB-2. The silty-sand interval in the upper member in GCB-1 appears to be the distal edge of the delta front. Overall, the general dip of the LS/SL sediments is south-southeast. The sand interval in the upper member appears to be a bar sand that is probably regressive, intertidal and defines the Lang Syne/Sawdust Landing regional unconformity.

The lower 10 feet $(3 \mathrm{~m})(230$ feet $(70.1 \mathrm{~m})$ to 240 feet $(73.2 \mathrm{~m})$ subsurface) of the LS/SL has an average permeability that exceeds 1 darcy. This interval is a well indurated clay with iron oxide staining and containing some pyrite. The measured permeability in this interval is probably due to microfracturing and the horizontal character of the iron oxide lamina. This interval is capped by a low permeability (less than 10 millidarcies) thinly bedded clay and silty clay. Overlying this clay, is a zone that transitions into a high permeability (greater than 1 darcy) that probably corresponds with the Lang. Syne/Sawdust Landing unconformity. In GCB-1, the combination of the underlying CBCU and the high permeability associated with the LS/SL unconformity create the zone with the highest TCE concentrations. The ability to track and map the Lang 
Syne/Sawdust Landing Formations and the unconformity zone suggests a way to possibly track halogenated solvents.

\section{Congaree Formation (Top Congaree)}

Unconformably overlying the Lang Syne/Sawdust Landing Formations are the assemblages of Early Eocene sediments that comprise the Fourmile Formation. It is lithologically difficult to distinguish these specific formations from the overlying lower middle Eocene Congaree Formation and the entire interval is generally referred to as the Congaree Formation (Aadland et al., 1995). Generally, the lower sands within the interval, when present, are considered equivalent to the Fourmile Formation while the upper sands, when present, are a portion of the Congaree Formation.

Generally, the depositional character of the Congaree in GCB-1,2,3 is similar. There is a slight increase in formation thickness from GCB-3 towards GCB-1 and 2 suggesting a seaward dip. The lithology of GCB-2 and 3 consists of sands, medium to coarse grained, interspersed with thin indurated clays. GCB-2 is dominantly sands with thin silts and a few clay lamina and GCB-3 is dominantly sand with thin clay and iron stained lamina. In GCB-1, there are lithified sand intervals 1.2 to 1.6 inches ( 30 to $40 \mathrm{~mm}$ ) thick and siliceous sandstones up to 2 inches $(50 \mathrm{~mm})$ thick. Well indurated clays are present as a central unit within the formation and are approximately 10 to 15 feet $(3$ to $4.6 \mathrm{~m})$ thick associated with silty clays and clayey silts.

Permeability zones are variable within the Congaree. In GCB-1, generally, within the formation, cleaner sand zones have higher permeability, approaching 500 millidarcies, and silty to clayey sands have permeability less that 5 millidarcies. Traces of PCE and TCE are generally seen in the thin sands that are immediately above the well indurated clays.

A shallow shelf to shoreline environments of deposition for the Congaree is evidenced by the dip directions in GCB-1. Basal Congaree sands (possible Fourmile or Fishburne Formation equivalents) generally rotate from a low angle south-southwest dip through a westward to north-northwest dip direction with an angle of $6^{\circ}$ to $8^{\circ}$ to a low angle northeasterly dip. The upper sand unit (probable Congaree Formation equivalent) has very high, up to $24^{\circ}$ dips generally striking to the northeast. The upper sand interval is most indicative of a possible dune sand with the high angle dips suggesting foreset bedding. The basal sands are most indicative of deltaic to near shore barriers and bars.

\section{Warley Hill Formation (Top WH)}

The Warley Hill Formation is present in GCB-1, 2 and 3. In GCB-3, the formation is generally geophysically indistinguishable from the over and underlying units, 
however, a subdued gamma pattern is similar to the patterns from GCB-1 and 2. In GCB-1, the Warley Hill consists of a sand with interspersed iron oxide modules overlying a silty sand to thinly laminated silty clay. The silty clay, and to some extent the silty sand, form the Gordon Confining Unit with measured log permeability of less than 50 millidarcies. Sands within the upper portion of the formation generally have permeability on the order of 100 to 200 md. In GCB-1, the dip directions within the Warley Hill are varied.

In GCB-2, the Warley Hill sediment character is similar to GCB-1 but the formation is locally exposed on the surface and has been affected by near surface processes. In GCB-3, the formation is generally sandier than GCB-1, and lacks the thin clay lamina and silty sands that constitute the confining unit.

Santee, Tobacco Road/Dry Branch. Upland Formations (Undifferentiated)

The Santee, Tobacco Road/Dry Branch, and Upland Formations are present only in GCB-1. The Santee consists of alternating thin units grading from pebbles and coarse grained sands upward to fine grained sands, all sub-angular to sub-rounded. Heavy minerals are interspersed throughout the units and sparse thinly laminated clays are present. The sands vary in grading from poorly sorted to moderately well sorted. Generally the dip direction of Santee sediments are randomly distributed. A near shore, fluvial to upper deltaic, possibly supratidal depositional environment is indicated by the sediment character. The upper interval of the Santee are generally accepted as forming the regional Santee unconformity of Aadland et al., (1995) and Fallaw and Price, (1995).

The Tobacco Road and Dry Branch Formations immediately overlie the Santee Formation. Both formations are principally sands and silty sands of similar size and grading distributions varying principally in color. The Tobacco Road Formation contains thin clay and silty clay intervals, principally in the base, with interspersed, thinly laminated silty sands. Heavy minerals are generally present.

The Dry Branch Formation consists principally of sands with thinly laminated clayey sands and iron oxide staining. Two intervals are distinct within the formation, an upper, poorly sorted, sub-angular to sub-rounded medium to coarse grained sand with abundant heavy minerals and a lower finer grained sand with thin clay lamina. The lower unit is indicative of fluvial channel to upper deltaic environment and the upper unit is more indicative of a distributary bar to shoreface environment.

The entire Dry Branch Formation is more permeable than the underlying Santee. The basal interval of the upper Dry Branch unit demonstrates measured permeability from 100 to 400 md while the basal unit of the lower unit, 
$\underline{\text { GCB-2 }}$

GCB-3

\begin{tabular}{|c|c|c|c|}
\hline $\begin{array}{l}\text { Upland } \\
\text { Depth Interval, ft (m) } \\
\text { Density, g/cc } \\
\text { Density Porosity, \% } \\
\text { Neutron Porosity, \% } \\
\text { Photo Electric Value } \\
\text { Resistivity, ohm/m } \\
\text { Permeability, md }\end{array}$ & $\begin{array}{c}32-48(9.5-14.6) \\
1.91(0.01) \\
44.47(0.85) \\
\text { no data } \\
2.31(0.01) \\
531(8) \\
45(3)\end{array}$ & not present, eroded & not present, eroded \\
\hline $\begin{array}{l}\text { TR/DB } \\
\text { Depth Interval, ft (m) } \\
\text { Density, g/cc } \\
\text { Density Porosity, \% } \\
\text { Neutron Porosity, \% } \\
\text { Photo Electric Value } \\
\text { Resistivity, ohm/m } \\
\text { Permeability, md }\end{array}$ & $\begin{array}{c}48-83(14.6-25.3) \\
1.94(0.006) \\
42.68(0.36) \\
\text { no data } \\
2.9(0.02) \\
543(5) \\
187(10)\end{array}$ & not present, eroded & not present, eroded \\
\hline $\begin{array}{l}\text { Santee } \\
\text { Depth Interval, } f(m) \\
\text { Density, g/cc } \\
\text { Density Porosity, \% } \\
\text { Neutron Porosity, \% } \\
\text { Photo Electric Value } \\
\text { Resistivity, ohm/m } \\
\text { Permeability, md }\end{array}$ & $\begin{array}{c}83-111(25.3-34) \\
1.92(0.02) \\
43.77(1.68) \\
33.29(2.27) \\
2.18(0.06) \\
352(12) \\
40(2) \\
\end{array}$ & not present, eroded & not present, eroded \\
\hline $\begin{array}{l}\text { WH to GCCZ } \\
\text { Depth Interval, ft (m) } \\
\text { Density, g/cc } \\
\text { Density Porosity, \% } \\
\text { Neutron Porosity, \% } \\
\text { Photo Electric Value } \\
\text { Resistivity, ohm/m } \\
\text { Permeability, md }\end{array}$ & $\begin{array}{c}111-123(34-37.5) \\
1.97(0.008) \\
40.78(0.5) \\
41.34(0.89) \\
1.80(0.04) \\
708(104) \\
88(5) \\
\end{array}$ & $\begin{array}{l}\text { behind pipe } \\
\text { no data }\end{array}$ & surface horizon \\
\hline $\begin{array}{l}\text { GCCZ } \\
\text { Depth Interval, ft (m) } \\
\text { Density, g/cc } \\
\text { Density Porosity, \% } \\
\text { Neutron Porosity, \% } \\
\text { Photo Electric Value } \\
\text { Resistivity, ohm/m } \\
\text { Permeability, md }\end{array}$ & $\begin{array}{c}123-133(37.5-40.5) \\
1.99(0.009) \\
39.92(0.56) \\
45.94(1.0) \\
2.31(0.06) \\
177(18) \\
27(3.1)\end{array}$ & $\begin{array}{l}\text { behind pipe } \\
\text { no data }\end{array}$ & $\begin{array}{c}35-47(10.6-14.3) \\
1.87(0.13) \\
37.56(0.77) \\
47.56(0.46) \\
1.40(0.04) \\
145(5) \\
110(9) \\
\end{array}$ \\
\hline
\end{tabular}

Table 2. Summary of sediment parameters derived from geophysical logs presented by stratigraphic unit. Geophysically derived values are followed by the standard deviation in parenthesis. Measured parameters and units are presented in the left hand column. "No data" means the interval was not geophysically logged because the formation was behind casing or too shallow for the logging tool length. 
A/M Area Advanced Geological Study, Part 2 of 3

WSRC-TR-97-0185

Interpretation of Geological Correlation Borings 1, 2, 3 in the

Rev. 0

A/M Area of the Savannah River Site, South Carolina (U)

Page 20 of 28

\begin{tabular}{|c|c|c|c|}
\hline Boring/Strata & GCB-1 & GCB-2 & GCB-3 \\
\hline $\begin{array}{l}\text { Steed Pond Aquifer } \\
\text { Depth Interval, ft (m) } \\
\text { Density, g/cc } \\
\text { Density Porosity, \% } \\
\text { Neutron Porosity, \% } \\
\text { Photo Electric Value } \\
\text { Resistivity, ohm/m } \\
\text { Permeability, md }\end{array}$ & $\begin{array}{c}133-220(40.5-67.3) \\
1.95(0.01) \\
42.36(0.73) \\
43.2(0.71) \\
1.74(0.01) \\
429(35) \\
273(20)\end{array}$ & $\begin{array}{l}\text { behind pipe } \\
\text { no data }\end{array}$ & $\begin{array}{c}47-139(14.3-42) \\
2.1(0.004) \\
38.7(0.26) \\
38.8(0.27) \\
1.38(0.006) \\
275(3) \\
127(12)\end{array}$ \\
\hline $\begin{array}{l}\text { LS/SL } \\
\text { Depth Interval, ft (m) } \\
\text { Density, g/cc } \\
\text { Density Porosity, \% } \\
\text { Neutron Porosity, \% } \\
\text { Photo Electric Value } \\
\text { Resistivity, ohm/m } \\
\text { Permeability, } \mathrm{md}\end{array}$ & $\begin{array}{c}220-251(67-76.5) \\
2.07(0.01) \\
34.9(0.87) \\
47.91(1.88) \\
1.93(0.5) \\
474(42) \\
1608(273)\end{array}$ & $\begin{array}{l}\text { behind pipe } \\
\text { no data }\end{array}$ & $\begin{array}{c}139-153(42.4-46.4) \\
2.1(0.005) \\
41.23(0.44) \\
40.17(0.32) \\
1.37(0.01) \\
43(1) \\
871(58)\end{array}$ \\
\hline $\begin{array}{l}\text { CBCU } \\
\text { Depth Interval, ft (m) } \\
\text { Density, g/cc } \\
\text { Density Porosity, \% } \\
\text { Neutron Porosity, \% } \\
\text { Photo Electric Value } \\
\text { Resistivity, ohm/m } \\
\text { Permeability, md }\end{array}$ & $\begin{array}{c}251-290(76.5-88.4) \\
2.05(0.004) \\
36.37(0.27) \\
57.47(0.51) \\
2.5(0.004) \\
211(6) \\
462(44)\end{array}$ & $\begin{array}{c}152-190(46.3-57.9) \\
2.05(0.004) \\
36.0(0.26) \\
59.87(0.61) \\
1.97(0.02) \\
379(46) \\
\text { no data }\end{array}$ & $\begin{array}{c}153-169(46.4-51.5) \\
2.6(0.06) \\
35.88(0.26) \\
44.2(0.64) \\
1.57(0.02) \\
250(5) \\
231(48)\end{array}$ \\
\hline $\begin{array}{l}\text { Steel Creek } \\
\text { Depth Interval, ft (m) } \\
\text { Density, g/cc } \\
\text { Density Porosity, \% } \\
\text { Neutron Porosity, \% } \\
\text { Photo Electric Value } \\
\text { Resistivity, ohm/m } \\
\text { Permeability, md }\end{array}$ & $\begin{array}{c}290-395(88.5-120.4) \\
2.07(0.003) \\
34.7(0.19) \\
43.5(0.47) \\
1.78(0.007) \\
1078(32) \\
8865(1295)\end{array}$ & $\begin{array}{c}190-271(57.9-82.6) \\
2.02(0.009) \\
37.84(0.56) \\
46.25(0.77) \\
1.57(0.01) \\
1224(50) \\
\text { no data }\end{array}$ & $\begin{array}{c}169-234(51.5-71.3) \\
2.1(0.001) \\
40.1(0.24) \\
42.96(0.31) \\
1.36(0.005) \\
339(22) \\
814(36)\end{array}$ \\
\hline $\begin{array}{l}\text { BC to MqBCU } \\
\text { Depth Interval, } \mathrm{ft}(\mathrm{m}) \\
\text { Density, g/cc } \\
\text { Density Porosity, \% } \\
\text { Neutron Porosity, \% } \\
\text { Photo Electric Value } \\
\text { Resistivity, ohm/m } \\
\text { Permeability, md }\end{array}$ & $\begin{array}{c}395-432(120.4-131.7) \\
2.12(0.002) \\
32.2(0.14) \\
38.81(0.29) \\
1.75(0.009) \\
1184(48) \\
\text { no data }\end{array}$ & $\begin{array}{c}271-390(82.6-118.9) \\
2.04(0.0) \\
36.77(0.23) \\
42.84(0.20) \\
1.56(0.005) \\
1416(43) \\
\text { no data }\end{array}$ & $\begin{array}{c}234-317(71.3-96.6) \\
1.97(0.002) \\
47.3(0.4) \\
44.48(0.14) \\
1.23(0.009) \\
266(1) \\
1441(63)\end{array}$ \\
\hline
\end{tabular}

Table 2 continued. 


\begin{tabular}{|c|c|c|c|}
\hline Boring/Strata & GCB-1 & GCB-2 & GCB-3 \\
\hline $\begin{array}{l}\text { MqBCU } \\
\text { Depth Interval, ft (m) } \\
\text { Density, g/cc } \\
\text { Density Porosity, \% } \\
\text { Neutron Porosity, \% } \\
\text { Photo Electric Value } \\
\text { Resistivity, ohm/m } \\
\text { Permeability, md }\end{array}$ & $\begin{array}{c}432-476(131.7-145.1) \\
2.11(0.005) \\
32.56(0.35) \\
47.6(0.84) \\
2.11(0.005) \\
786.7(64) \\
\text { no data } \\
\end{array}$ & $\begin{array}{c}390-429(118.9-130.8) \\
2.08(0.008) \\
34.40(0.48) \\
51.47(0.87) \\
1.72(0.02) \\
908(69) \\
\text { no data } \\
\end{array}$ & $\begin{array}{c}317-352 \text { (96.6-107.3) } \\
\text { no data } \\
\text { no data } \\
\text { no data } \\
\text { no data } \\
\text { no data } \\
2048(219) \\
\end{array}$ \\
\hline $\begin{array}{l}\text { MqBCU to Mid. } \\
\text { Depth Interval, ft (m) } \\
\text { Density, g/cc } \\
\text { Density Porosity, \% } \\
\text { Neutron Porosity, \% } \\
\text { Photo Electric Value } \\
\text { Resistivity, ohm/m } \\
\text { Permeability, md }\end{array}$ & $\begin{array}{c}476-582(145.1-177.4) \\
2.13(0.004) \\
31.56(0.28) \\
39.7(0.29) \\
1.76(0.01) \\
1083(33) \\
\text { no data } \\
\end{array}$ & $\begin{array}{c}429-515(130.8-157) \\
2.08(0.006) \\
34.2(0.39) \\
42.91(0.24) \\
1.6(0.01) \\
1350(44) \\
\text { no data } \\
\end{array}$ & $\begin{array}{c}\text { 352-472 (107.3-143.9) } \\
\text { no data } \\
\text { no data } \\
\text { no data } \\
\text { no data } \\
\text { no data } \\
820(74) \\
\end{array}$ \\
\hline $\begin{array}{l}\text { Mid to CF } \\
\text { Depth Interval, ft (m) } \\
\text { Density, g/cc } \\
\text { Density Porosity, \% } \\
\text { Neutron Porosity, \% } \\
\text { Photo Electric Value } \\
\text { Resistivity, ohm/m } \\
\text { Permeability, md } \\
\end{array}$ & $\begin{array}{c}582-696(177.4-212.1) \\
2.13(0.004) \\
31.1(0.26) \\
34.92(0.22) \\
1.72(0.006) \\
1141(32) \\
\text { no data } \\
\end{array}$ & $\begin{array}{c}515-626(157-190.8) \\
2.13(0.006) \\
31.38(0.37) \\
36.79(0.30) \\
1.63(0.008) \\
901(48) \\
\text { no data } \\
\end{array}$ & $\begin{array}{c}472-597(143.9-182) \\
\text { no data } \\
\text { no data } \\
\text { no data } \\
\text { no data } \\
\text { no data } \\
154(10)\end{array}$ \\
\hline $\begin{array}{l}\text { Cape Fear } \\
\text { Depth Interval, } \mathrm{ft}(\mathrm{m}) \\
\text { Density, g/cc } \\
\text { Density Porosity, \% } \\
\text { Neutron Porosity, \% } \\
\text { Photo Electric Value } \\
\text { Resistivity, ohm/m } \\
\text { Permeability, md } \\
\end{array}$ & $\begin{array}{c}696-728(212.1-221.9) \\
2.22(0.01) \\
25.94(0.69) \\
40.69(0.94) \\
2.14(0.04) \\
74.94(8.15) \\
\text { no data } \\
\end{array}$ & $\begin{array}{c}626-650(190.8-198.1) \\
2.19(0.01) \\
27.44(0.61) \\
38.45(0.13) \\
1.99(0.04) \\
80(8) \\
\text { no data } \\
\end{array}$ & $\begin{array}{c}597-618 \text { (182-188.4) } \\
\text { no data } \\
\text { no data } \\
\text { no data } \\
\text { no data } \\
\text { no data } \\
7(2)\end{array}$ \\
\hline $\begin{array}{l}\text { Saprolite } \\
\text { Depth Interval, ft (m) } \\
\text { Density, g/cc } \\
\text { Density Porosity, \% } \\
\text { Neutron Porosity, \% } \\
\text { Photo Electric Value } \\
\text { Resistivity, ohm/m } \\
\text { Permeability, md } \\
\end{array}$ & $\begin{array}{c}728-757(221.9-230.7) \\
2.16(0.02) \\
29.55(1.55) \\
44.59(1.39) \\
2.94(0.04) \\
7.88(0.69) \\
\text { no data } \\
\end{array}$ & $\begin{array}{c}650-665(198.1-202.7) \\
2.2(0.02) \\
38.19(0.16) \\
53.26(0.10) \\
2.74(0.09) \\
7(0.3) \\
\text { no data } \\
\end{array}$ & $\begin{array}{c}\text { 618-631 (188.4-192.3) } \\
\text { no data } \\
\text { no data } \\
\text { no data } \\
\text { no data } \\
\text { no data } \\
\text { no data } \\
\end{array}$ \\
\hline $\begin{array}{l}\text { Crystalline } \\
\text { Depth Interval, } \mathrm{ft}(\mathrm{m}) \\
\text { Density, g/cc } \\
\text { Density Porosity, \% } \\
\text { Neutron Porosity, \% } \\
\text { Photo Electric Value } \\
\text { Resistivity, ohm/m } \\
\text { Permeability, md } \\
\end{array}$ & $\begin{array}{c}757-774(230.7-235.9) \\
2.51(0.005) \\
8.42(0.35) \\
23.57(0.68) \\
3.1(0.02) \\
14.41(0.24) \\
\text { no data } \\
\end{array}$ & $\begin{array}{c}666-676(202.7-206) \\
2.50(0.01) \\
8.82(1.06) \\
38.8(1.4) \\
3.98(0.14) \\
9.9(0.04) \\
\text { no data } \\
\end{array}$ & $\begin{array}{c}631-722(192.3-220.1) \\
\text { no data } \\
\text { no data } \\
\text { no data } \\
\text { no data } \\
\text { no data } \\
\text { no data } \\
\end{array}$ \\
\hline Total Depth, ft (m) & $802(244.4)$ & $684(208.5)$ & $722(220.1)$ \\
\hline
\end{tabular}

Table 2 continued. 
immediately overlying the Santee unconformity, has log derived permeability approaching $600 \mathrm{md}$.

The Upland Formation is present on the surface to a depth of approximately 17 feet $(5.2 \mathrm{~m})$ in GCB-1. The formation is similar to the underlying Tobacco Road and consists of moderately to well sorted, sub-angular to sub-rounded sands and silty sands with interspersed thin clay lamina. The well sorted sands are immediately overlying the Tobacco Road Formation and form a fining upwards fluvial channel with abundant heavy minerals and micas. Shallower sediments of the Upland have been affected by near surface processes.

\section{HYDROGEOLOGIC RELATIONSHIPS}

Table 2 provides a unit to unit comparison of basic hydrogeological parameters such as permeability and porosity. In general, GCB-1 and 2 contain intervals with sufficient amounts of clay or fine grained silty material to provide localized confining units. GCB-3 contains only minimal clay amounts in thin intervals with minimal confining capability. The relative amounts of sand in each location is shown on a sand-mud (clay)-gravel ternary diagram in Figure 6. For GCB-1, in blue, there are dominant sands intervals but also dominant mud (clay) zones. GCB-2, in red, is dominantly sand but with a higher fraction of clay and fewer distinct clays. Clays, and gravels, in GCB-3, in green, are more dispersed with minimal to no distinct clays. Most confining units in GCB-3 are silty clays to clayey silts. Gravels are more prominent and persistent in GCB-3 supporting the updip environment of deposition interpretation for this boring. Based on the ternary relationships of Figure 6, GCB-1 is depositionally more downdip or seaward than GCB-2, and both are depositionally more marine than GCB-3.

Figures 7 and 8 are z-plots of GCB-1 and 3 respectively. These figures plot the gamma values versus the percent clays (muds) versus the deep induction resistivity. GCB-1 is representative of GCB-2 (refer to ternary distribution on Figure 6) so only GCB-1 is plotted and only the upper aquifers are shown for GCB-3. This combination of data provides a graphical display of aquifers (relatively clean sands with freshwater resistivity) versus confining units (high mud content intervals with lower mineral resistivity), both in depth. Highlighted areas on the graph are presented relative to depth and gamma signature to indicate confining zones. Relative confining unit (or conversely, an aquifer unit) location and thickness may be inferred from these data. Competent confining units are seen in GCB-1 (and therefore for GCB-2) and thin, minimal confining zones are seen in GCB-3. As noted on Figure 6, the confining units defined by the z-plot for GCB-3 contain higher amounts of silty material than do the confining units in GCB-1. 


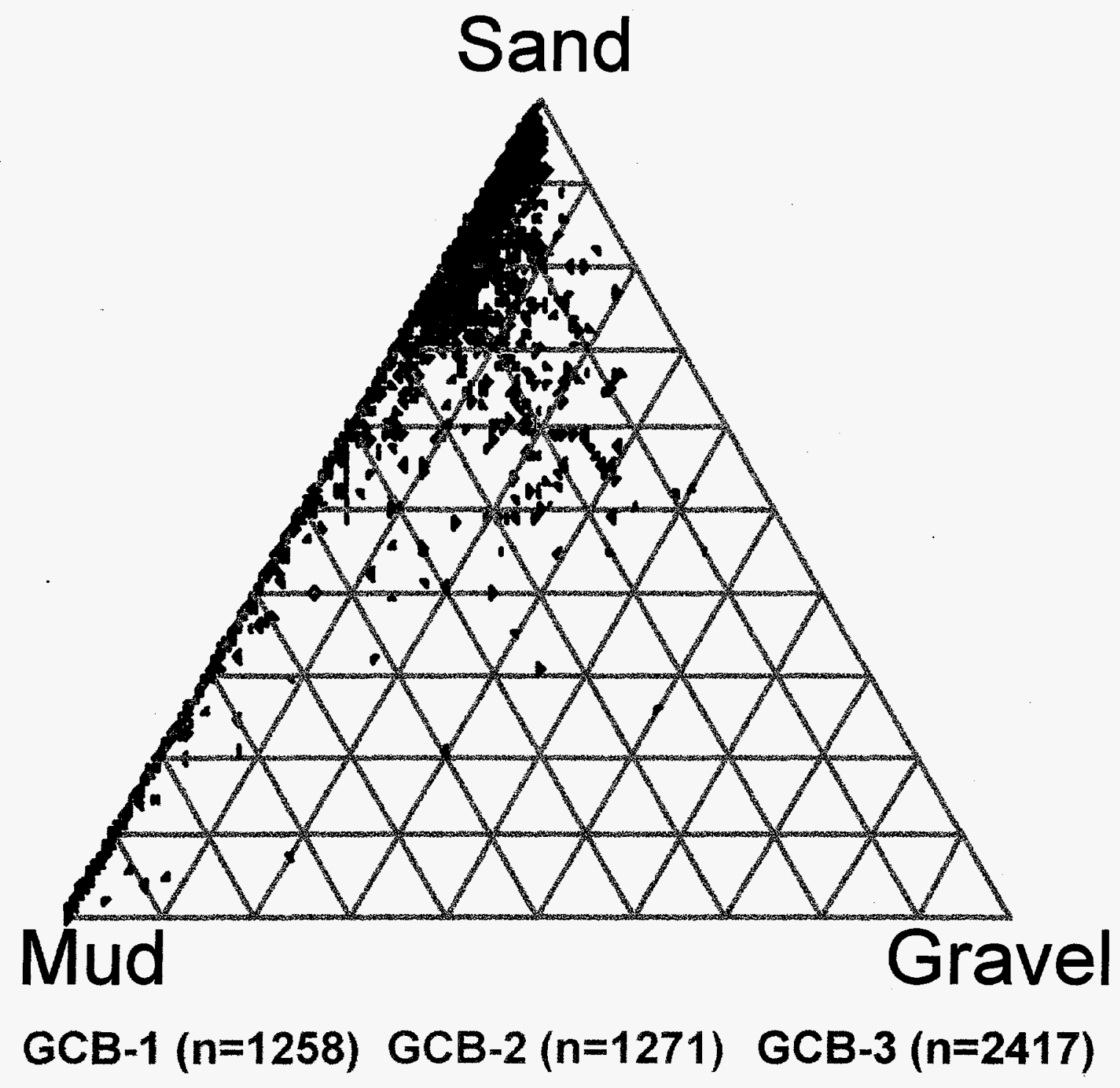

Figure 6. Ternary diagram of sand, mud and gravel distribution in GCB-1,2,3. GCB-1 is in blue, GCB-2 in red and GCB-3 in green. GCB-1 has dominant sand lithologies (large clusters of blue near the sand node) with lessor dominant but distinct mud (clay) facies (cluster of blue near mud node). GCB-2 is predominantly sand but is more dispersed towards the mud and gravel nodes than GCB-1. GCB-3 is also dominantly sand but is also more dispersed towards the gravel node with only a minor mud (clay) component. Data were derived from core lab analysis. 


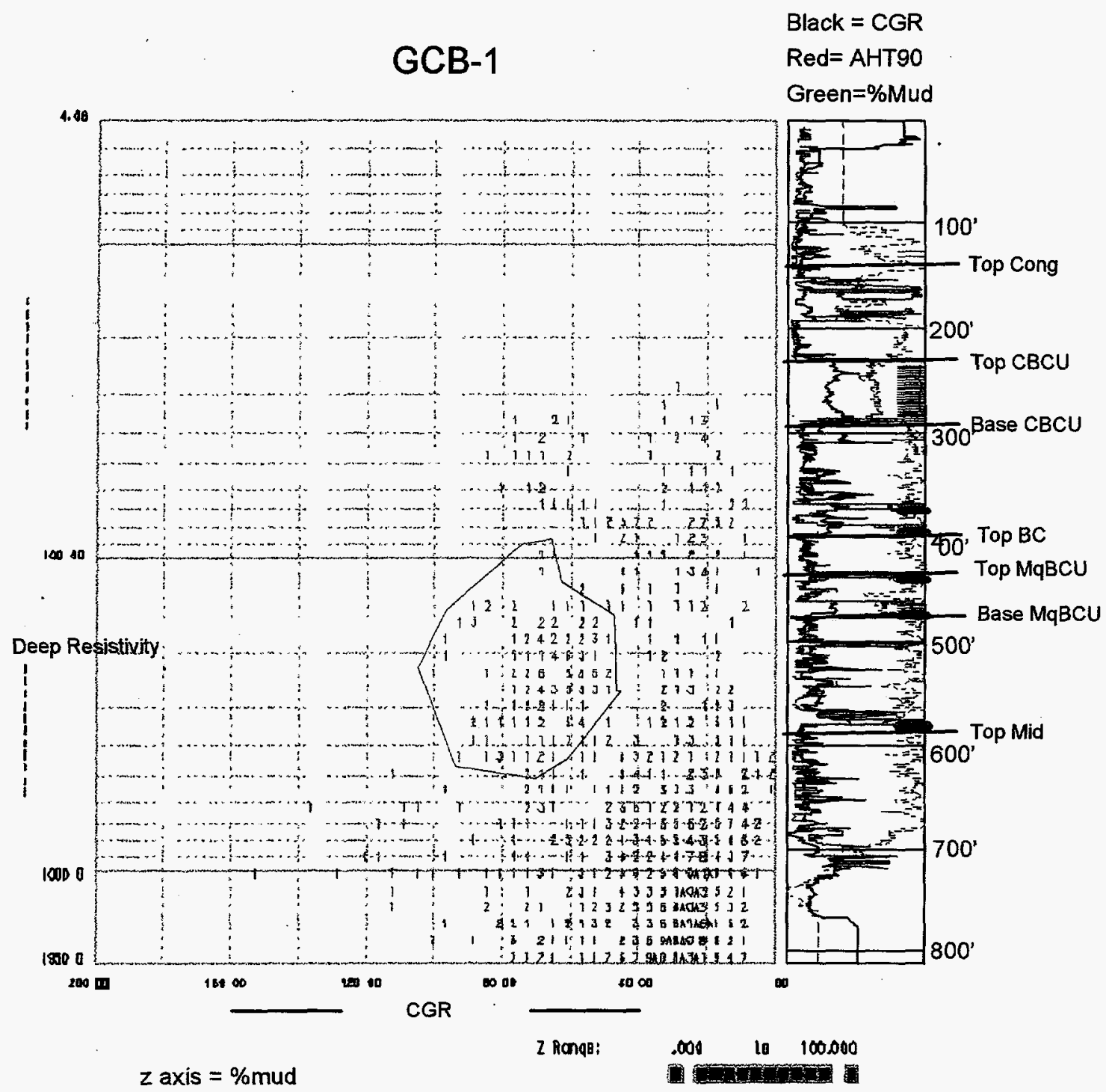

Figure 7. Z-plot of GCB-1 using deep induction resistivity, percent mud (clay) and the calculated gamma ray response. The area outlined in purple reflects relatively low resistivity, moderate gamma counts and high percentages of mud, all indicators of potential confining units. The purple zones on the geophysical log plot to the right correspond to values outlined on the graph and indicate potential depths and stratigraphic locations of confining units. 


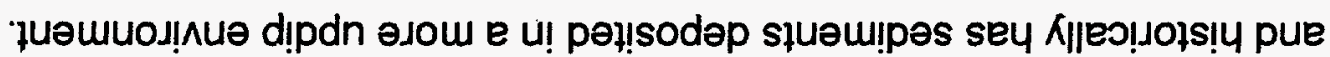

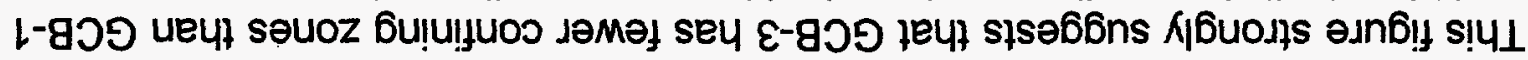

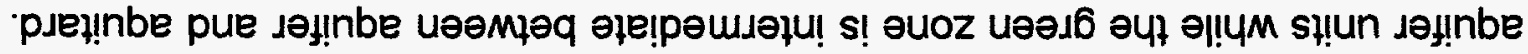

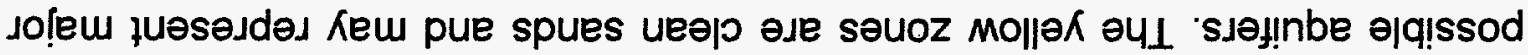
səu!fəp чग!

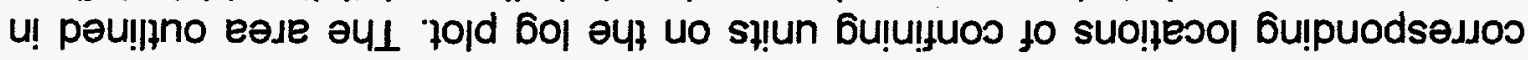

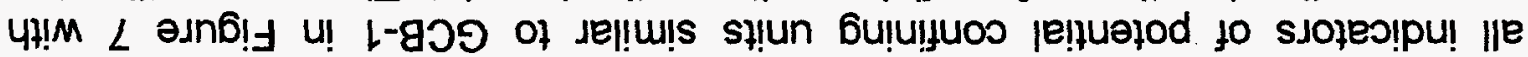

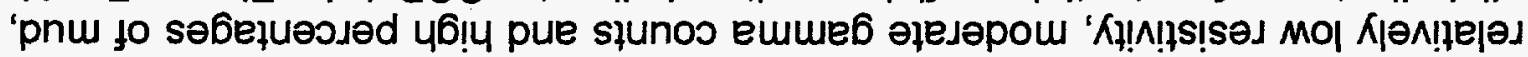

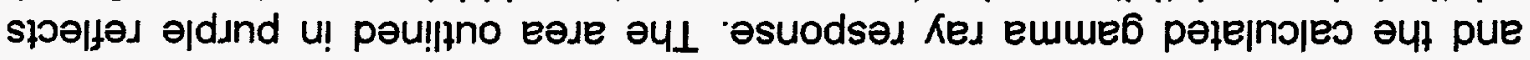

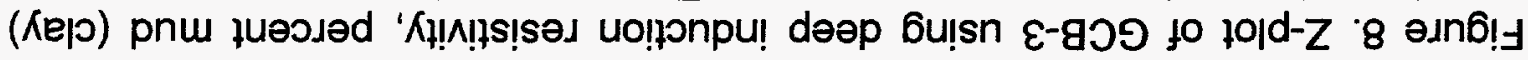

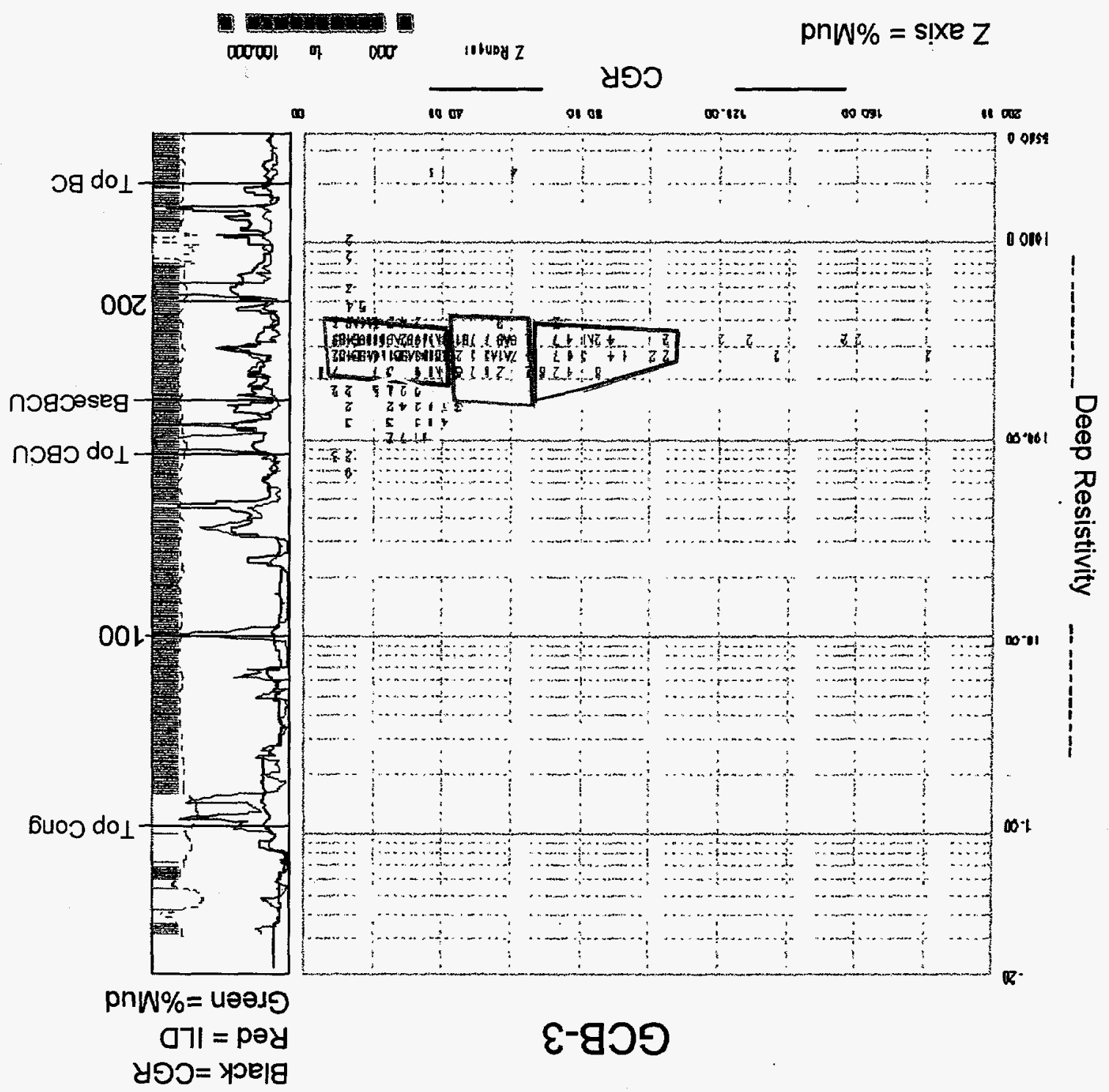

82 fo $\varsigma$ a6ed 0 'งәу S\&LO-L6-YעL-JYSM

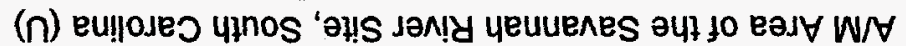

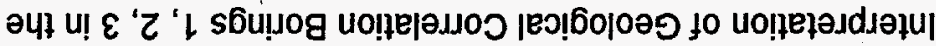

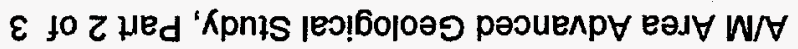




\section{SUMMARY}

The interpretation of the lithostratigraphy of GCB-1,2,3 suggests that GCB-3 contains sediments that were generally deposited in a more near-shore, subtidal to fluvial, updip environment than sediments in GCB-1 or 2. GCB-1 contains lithologies that are deeper water, subtidal to deltaic while GCB-2 has similar sediments that were deposited in a slightly shallower environment. The present relationship between GCB-1,2,3 indicates that GCB-2 is structurally lower relative to GCB-1 and 3 . Correlation of strata between the borings suggests that basement structural events continued through the Paleocene and are terminated by the Lang Syne/Sawdust Landing unconformity. Thickening of sediments within the structurally low depression of GCB-2 after the Lang Syne/Sawdust Landing unconformity suggest structural reactivation during the Eocene.

In general, GCB-1 and 2 contain intervals with sufficient amounts of clay or fine grained silty material to provide localized confining units throughout the stratigraphic column. GCB-3 contains only minimal clay amounts in thin intervals with minimal confining capability in Paleocene and Eocene formations, while thicker clays, with larger amounts of silt, are present in the Cretaceous age materials.

The use of advanced geophysical logging has proven highly successful in the GCB borings. Multi-spectral gamma ray measurements allowed for the delineation of clays versus naturally radioactive sands providing a more precise aquitard definition. Sand units with similar spectral signatures were correlated with more confidence. The use of multiple porosity tools such as the density and neutron porosity provided aquifer and confining unit porosity based on differing physical measurements. The density utilizes bulk volume of sediments while the neutron utilizes fluid content. The comparison of the measured porosity from these tools allow for a confident porosity value to be determined. Focused induction resistivity logs provided a detailed definition of vertical variations in resistivity associated with hydrogeological units. Zones of greater or lessor fluid movement could be determined as well as the influence of heavy minerals. The highly advanced magnetic resonance logging provided for a precise measurement of in-situ permeability and defined zones of greater or lessor flow potential. The borehole sonic data provided a distinct correlation between surface reflection seismic data and lithostratigraphic response. The use of these logs combined allowed for a more precise definition lithostratigraphy and hydrostratigraphy and for the interpretation of depositional setting. 
The analysis of laboratory core, field core and geophysical log derived data in the GCB borings provides a powerful tool for a comprehensive geological analysis of subsurface of the Upper Three Runs Watershed. Environments of deposition, structural and stratigraphic correlation and aquifer characteristics may be derived form a combination of these data, leading to a more powerful predictive tool of groundwater flow supporting remedial efforts. 


\section{REFERENCES}

Aadland, R. K., J. A. Gellici and P. A. Thayer, 1995, Hydrogeologic framework of West-Central South Carolina, South Carolina Department of Natural Resources, Water Resources Division, Report 5, 200 p.

Busch, D. A. and D. A. Link, 1985, Exploration Methods for Sandstone Reservoirs, OGCI Publications, Tulsa, 327 p.

Cumbest, R. J., and V. Price, 1989, Continued extension of the Dunbarton Basin: an explanation for faulting in the coastal plain of South Carolina: WSRC-RP-89-1263.

Fallaw, W. C. and V. Price, 1995, Stratigraphy of the Savannah River Site and vicinity. Southeastern Geology, Vol. 35, No. 1, pp. 21-58.

Reineck, H. E. and I. B. Singh, 1980, Depositional sedimentary environments, 2nd ed. Springer-Verlag, New York, $549 \mathrm{p}$.

Schlumberger, 1989, Log Interpretation Principles/Applications, Schlumberger Educational Services, Houston, 213 p.

Serra, O., 1985, Sedimentary Environments from Wireline Logs, Schlumberger Educational Services, Houston, $211 \mathrm{p}$. 
WSRC-RP-97-0185

Revision 0

June, 1997

Appendix A

USGS Field Core Descriptions for GCB-1, 2, 3 
WSRC-RP-97-0185

Revision 0

June, 1997

This page intentionally left blank. 
Core Hole: \# GCB-1

\section{8-25-96}

Run $1 \quad 500-503$

Run $2 \quad 503-505$

Recovered:

Run $3 \quad 505-512$

2.3' 500-502.3

$1.5 \quad 503-504.5$

$5.6 \quad 505-510.6$

Run $4 \quad 512-517$

$1.7 \quad 512-513.7$

Run $5 \quad 517-520$

$1.1 \quad 517-518.1$

Run $6 \quad 520-525$

$4.6 \quad 520-524.6$

Run $7 \quad 525-528$

1.9. 525-526.9

Run $8 \quad 528-533$

$2.9 \quad 528-530.9$

\begin{tabular}{|c|c|c|c|}
\hline \multicolumn{4}{|l|}{$8-26-96$} \\
\hline Run 9 & $533-536.2$ & 2.7 & $533-535.7$ \\
\hline Run 10 & $536.2-540$ & 1.2 & $536.2-537.4$ \\
\hline Run 11 & $540-540.5$ & $100 \%$ & \\
\hline Run 12 & $540.5-544$ & 2.7 & $540.5-543.2$ \\
\hline Run 13 & $544-547$ & $100 \%$ & \\
\hline Run 14 & $547-552$ & 4.7 & $547-551.7$ \\
\hline Run 15 & $552-557$ & 4.1 & $552-556.1$ \\
\hline Run 16 & $557-560.5$ & 2.3 & $557-559.3$ \\
\hline Run 17 & $560.5-562$ & \multicolumn{2}{|c|}{ no recovery } \\
\hline Run 18 & $562-564$ & 1.7 & $562-563.7$ \\
\hline Run 19 & $564-566.3$ & 1.8 & $564-565.8$ \\
\hline Run 20 & $566.3-569$ & 2.6 & $566.3-568.9$ \\
\hline Run 21 & $569-572.5$ & $100 \%$ & \\
\hline Run 22 & $572.5-574$ & $100 \%$ & \\
\hline Run 23 & $574-578$ & $100 \%$ & \\
\hline Run 24 & $578-582$ & 3.3 & $578-581.3$ \\
\hline Run 25 & $582-585$ & 2 & $582-584$ \\
\hline Run 26 & $585-592$ & 6.9 & $585-591.9$ \\
\hline Run 27 & $592-596$ & 3.6 & $592-595.6$ \\
\hline Run 28 & $596-599.5$ & 2.6 & $596-598.6$ \\
\hline Run 29 & $599.5-600$ & \multicolumn{2}{|c|}{ no recovery } \\
\hline Run 30 & $600-606$ & \multicolumn{2}{|c|}{ no recovery } \\
\hline Run 31 & $606-607$ & \multicolumn{2}{|c|}{ no recovery } \\
\hline \multicolumn{4}{|l|}{$8-27-96$} \\
\hline Run 32 & $607-609$ & 1.1 & $607-608.1$ \\
\hline Run 33 & $609-612$ & 2.2 & $609-611.2$ \\
\hline Run 34 & $612-616$ & 3.6 & $612-615.6$ \\
\hline Run 35 & $616-620$ & 3 & $616-619$ \\
\hline Run 36 & $620-620.5$ & $100 \%$ & \\
\hline Run 37 & $620.5-627$ & 3.2 & $620.5-623.7$ \\
\hline
\end{tabular}


Recovered:

\begin{tabular}{|c|c|c|c|}
\hline Run 38 & $627-632$ & 2.7 & $627-629.7$ \\
\hline Run 39 & $632-633.5$ & 1 & $632-633$ \\
\hline Run 40 & $633.5-636$ & 1.4 & $633.5-634.9$ \\
\hline Run 41 & 636-639 & 2.9 & $636-638.9$ \\
\hline Run 42 & $639-644$ & 4.1 & $639-643.1$ \\
\hline Run 43 & $644-647$ & 2.1 & $644-646.1$ \\
\hline Run 44 & $647-650$ & 2.3 & $647-649.3$ \\
\hline Run .45 & $650-653$ & 2.4 & $650-652.4$ \\
\hline Run 46 & $653-656$ & .5 & $653-653.5$ \\
\hline Run 47 & $656-660$ & 3 & $656-659$ \\
\hline Run 48 & $660-666$ & 3.1 & $660-663.1$ \\
\hline \multicolumn{4}{|l|}{$8-28-96$} \\
\hline Run 49 & $666-670$ & 2.4 & $666-668.4$ \\
\hline Run 50 & $670-673$ & 1.8 & $670-671.8$ \\
\hline Run 51 & $673-678$ & 2.8 & $673-675.8$ \\
\hline Run 52 & $678-679$ & .8 & $678-688.8$ \\
\hline Run 53 & $679-680$ & $100 \%$ & \\
\hline Run 54 & $680-682$ & 1.8 & $680-681.8$ \\
\hline Run 55 & $682-683$ & .6 & $682-682.6$ \\
\hline Run 56 & $683-686$ & $100 \%$ & \\
\hline Run 57 & $686-687$ & \multicolumn{2}{|c|}{ no recovery } \\
\hline Run 58 & $687-689.5$ & 2.3 & $687-689.3$ \\
\hline Run 59 & $689.5-689.8$ & \multicolumn{2}{|c|}{ no recovery } \\
\hline Run 60 & $689.8-692$ & 1.3 & $689.8-691.1$ \\
\hline Run 61 & $692-695$ & 2.9 & $692-694.9$ \\
\hline Run 62 & 695-698 & $100 \%$ & \\
\hline Run 63 & $698-700$ & $100 \%$ & \\
\hline Run 64 & $700-706$ & 3.7 & $700-703.7$ \\
\hline \multicolumn{4}{|l|}{$8-29-96$} \\
\hline Run 65 & $706-708.5$ & 2.4 & $706-708.4$ \\
\hline Run 66 & $708.5-712$ & 3.4 & $708.5-711.9$ \\
\hline Run 67 & $712-713$ & .8 & $712-712.8$ \\
\hline Run 68 & 713-715 & 1.5 & $713.5-715$ \\
\hline Run 69 & $715-716$ & $100 \%$ & \\
\hline Run 70 & $716-718$ & $100 \%$ & \\
\hline Run 71 & $718-722$ & $100 \%$ & \\
\hline Run 72 & $722-723$ & $100 \%$ & \\
\hline Run 73 & $723-727$ & 3.9 & $723-726.9$ \\
\hline Run 74 & $727-731$ & $100 \%$ & \\
\hline Run 75 & $731-736$ & $100 \%$ & \\
\hline Run 76 & $736-742.4$ & $100 \%$ & \\
\hline Run 77 & $742.4-743$ & $100 \%$ & \\
\hline
\end{tabular}




\begin{tabular}{|c|c|c|c|}
\hline & & & vered \\
\hline Ruñ 78 & $743-745$ & $100 \%$ & \\
\hline Run 79 & $745-747.5$ & $100 \%$ & \\
\hline Run 80 & $747.5-752$ & 4.3 & $747.5-751.8$ \\
\hline $8-31-96$ & & & \\
\hline Run 81 & $752-757$ & 4.8 & $752-756.8$ \\
\hline Run 82 & $757-761$ & $100 \%$ & \\
\hline Run 83 & $761-762$ & $100 \%$ & \\
\hline Run 84 & $762-762.3$ & $100 \%$ & \\
\hline Run 85 & $762.3-766.3$ & $100 \%$ & \\
\hline Run 86 & $766.3-769.3$ & $100 \%$ & \\
\hline Run 87 & $769.3-771.5$ & $100 \%$ & \\
\hline Run 88 & $771.5-774$ & $100 \%$ & \\
\hline $9-1-96$ & & & \\
\hline Run 89 & $774-781$ & $100 \%$ & \\
\hline Run 90 & $781-785.8$ & $100 \%$ & \\
\hline Run 91 & $785.8-787.4$ & $100 \%$ & \\
\hline $9-2-96$ & & & \\
\hline Run 92 & $787.4-791$ & $100 \%$ & \\
\hline Run 93 & $791-799$ & $100 \%$ & \\
\hline Run 94 & $799-802.3$ & & \\
\hline $9-3-96$ & & & \\
\hline Run 95 & $802.3-802.7$ & & \\
\hline
\end{tabular}

Total: $79 \%$ recovered 


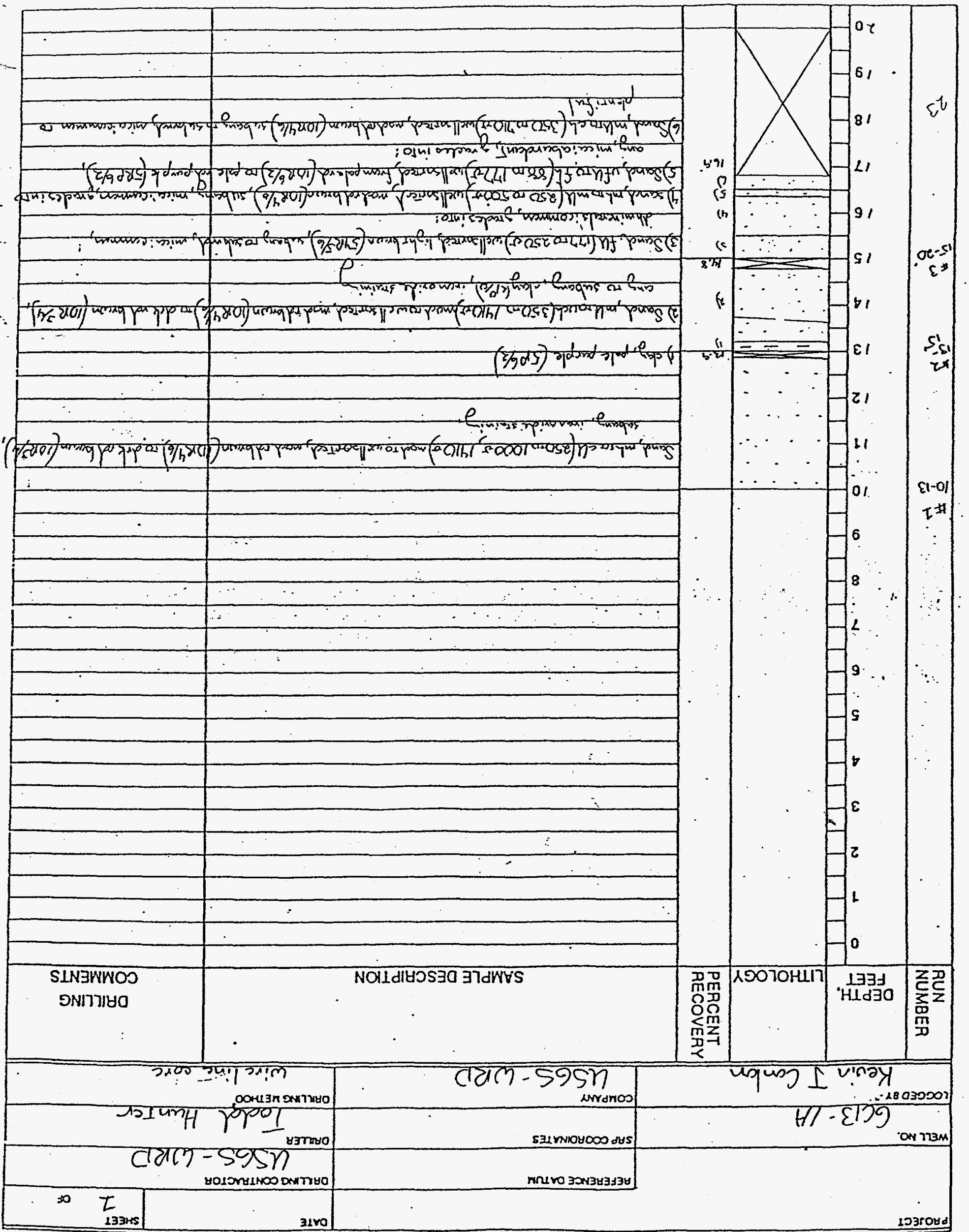


FIELD GEOLOGIĊ LOG

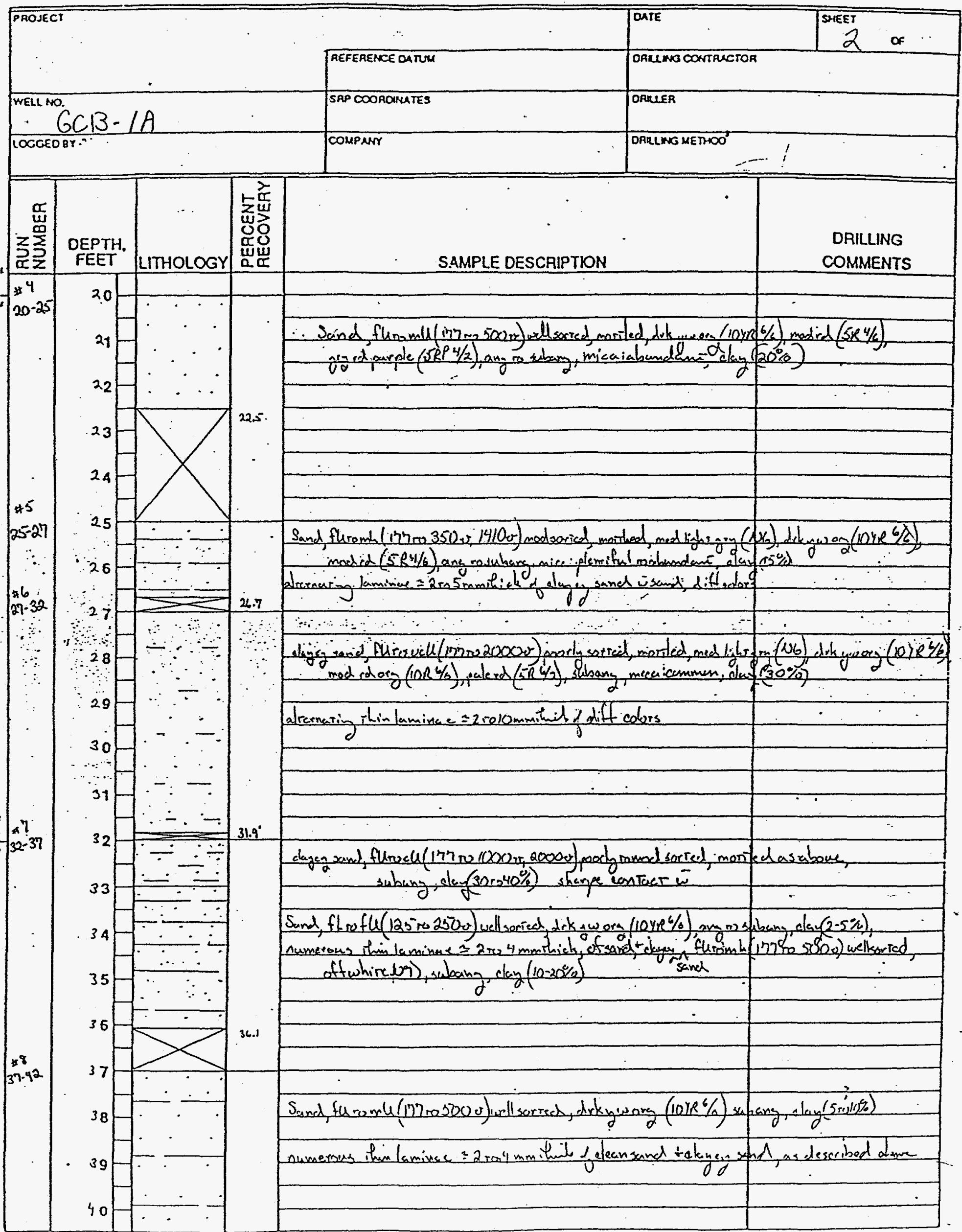


OSR 20.3

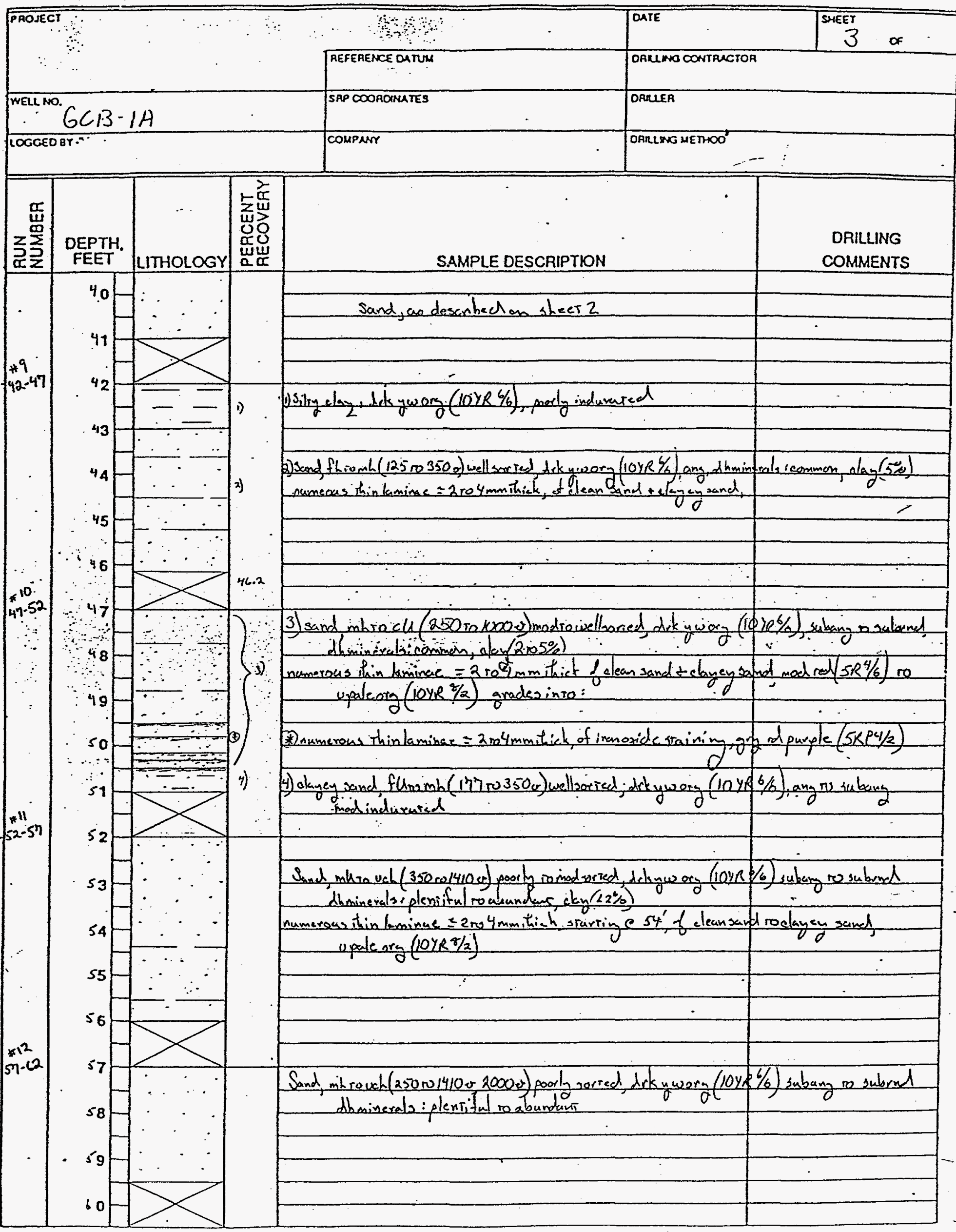


FIELD GEOLOGIC LOG

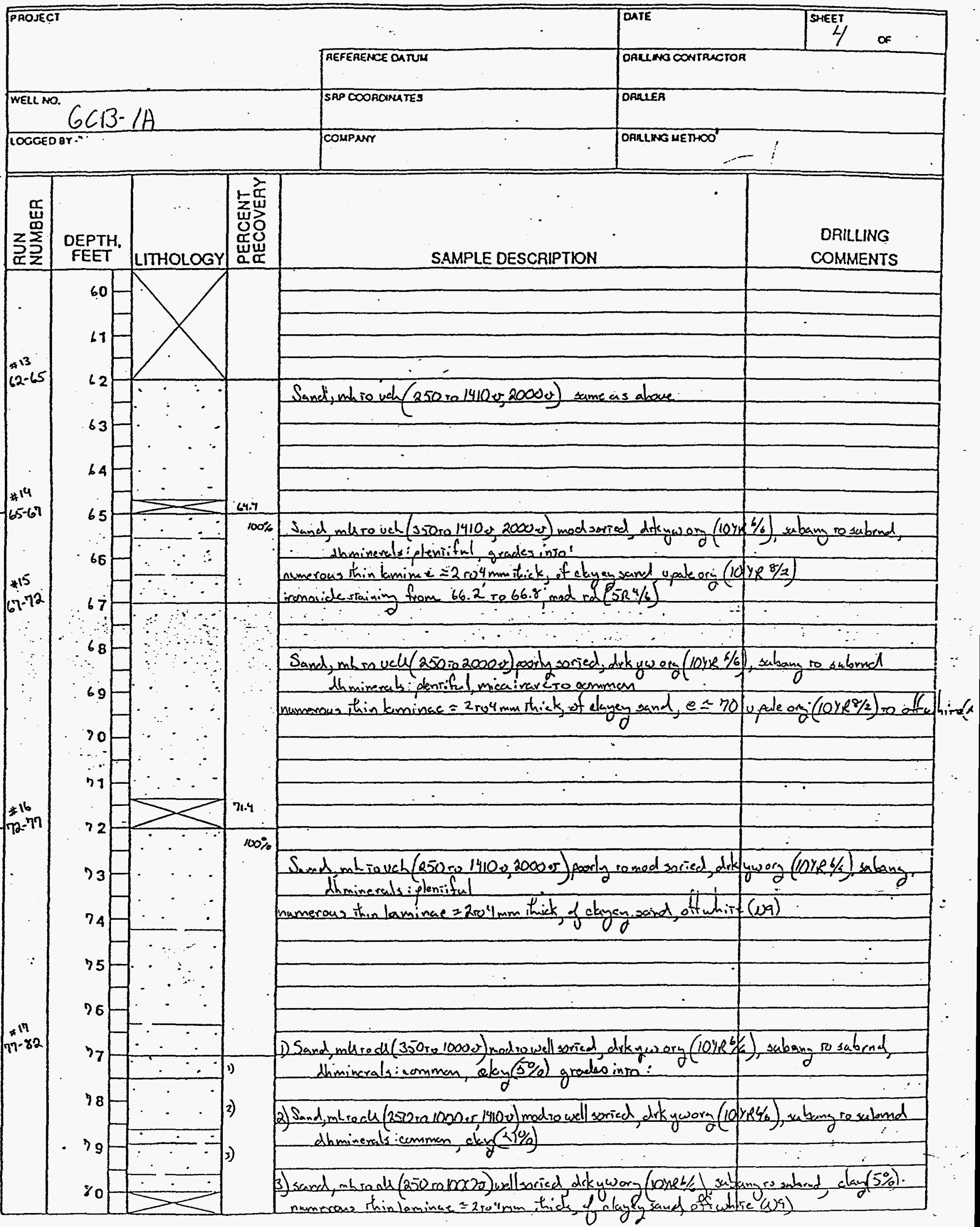




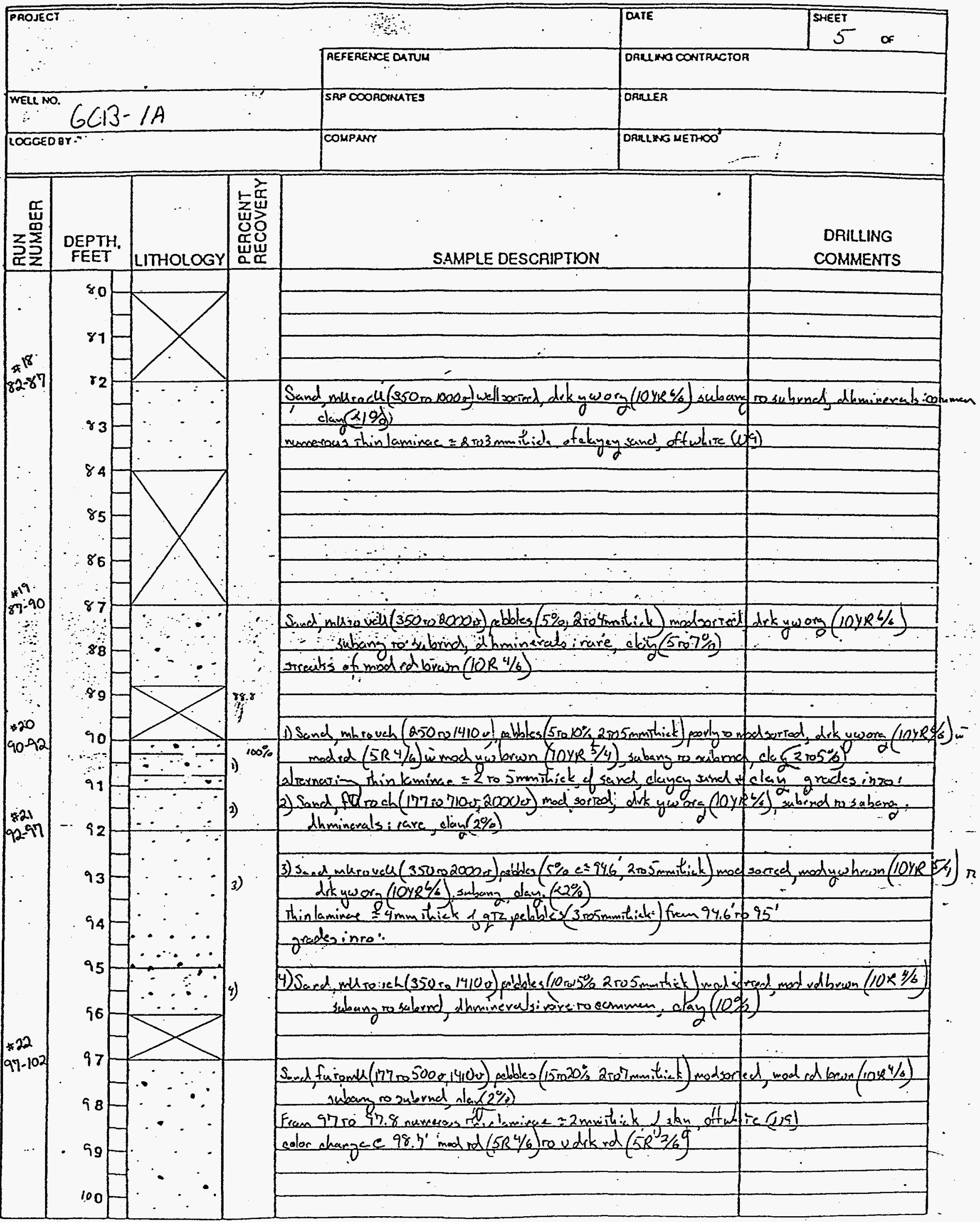


FIELD GEOLOGIC LOG

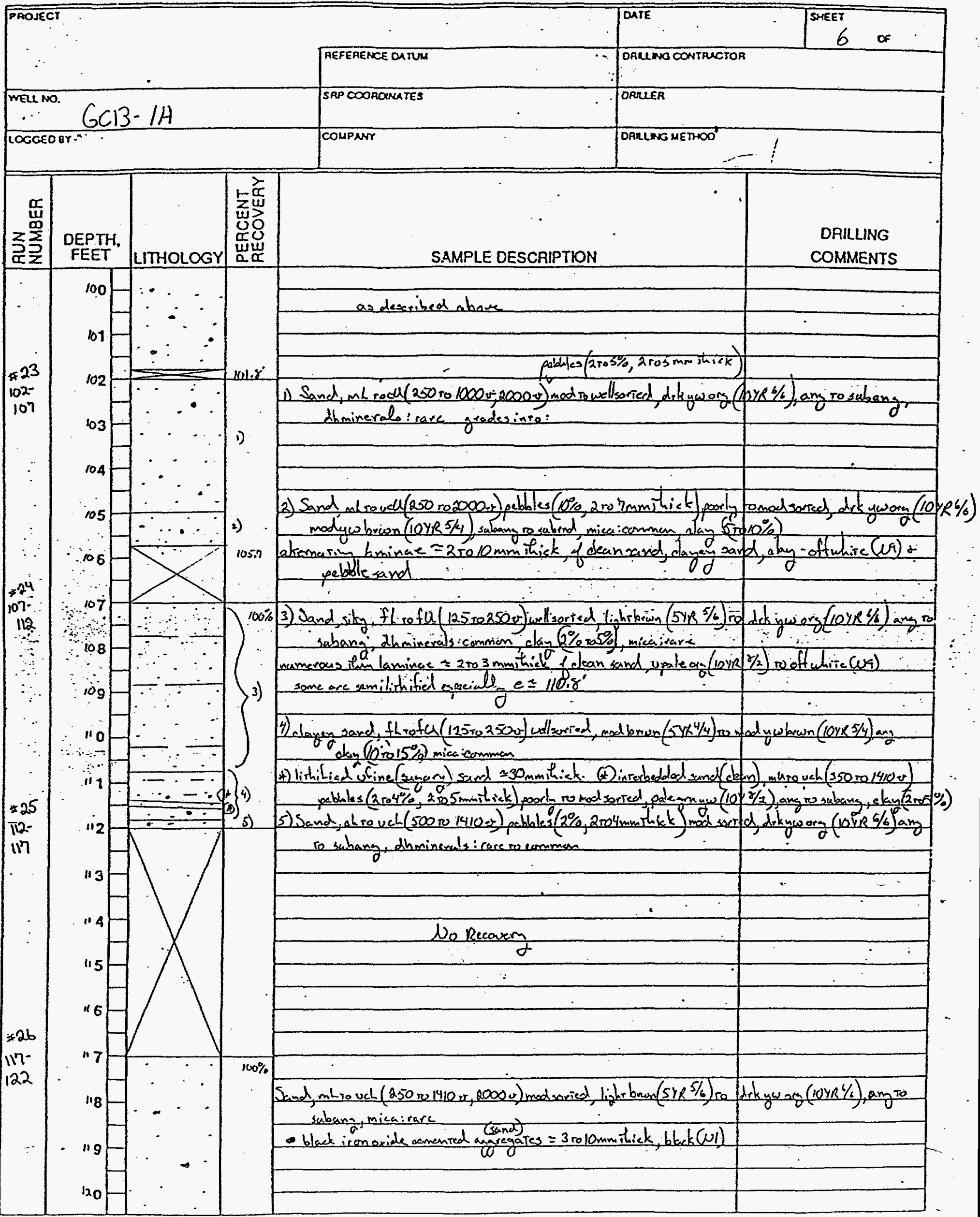




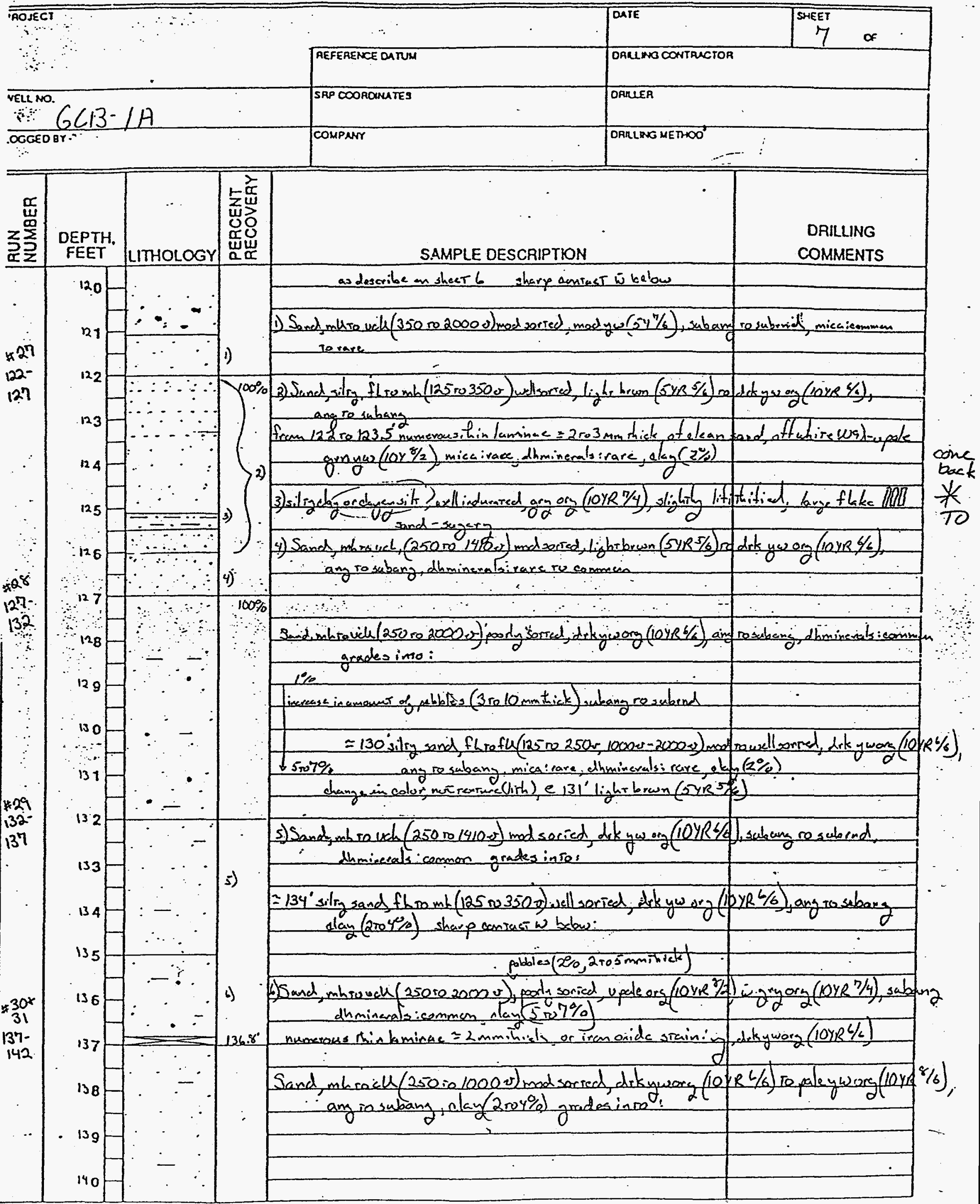


ol

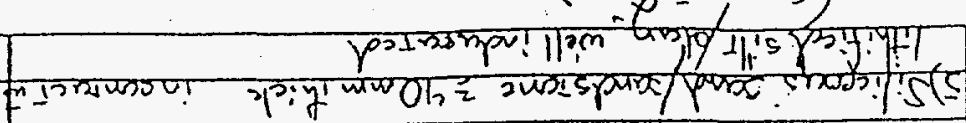

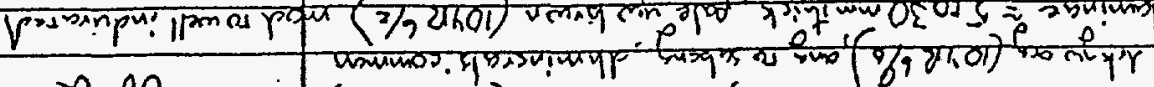
वी

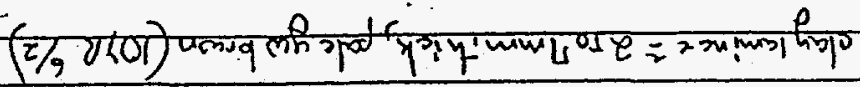

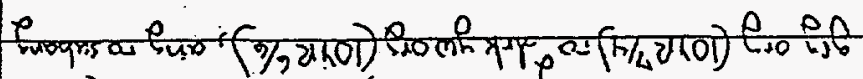

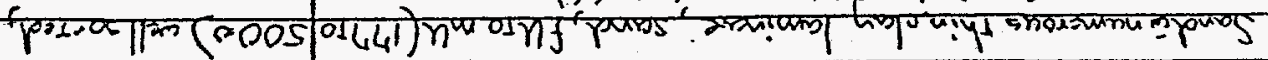

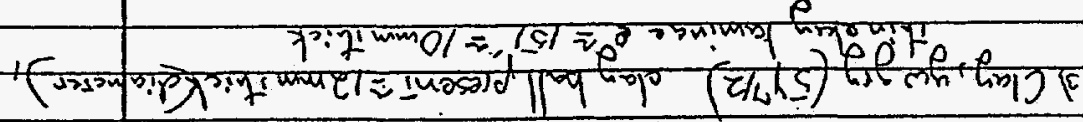

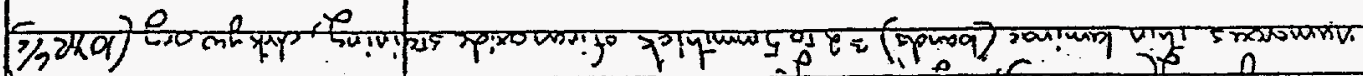

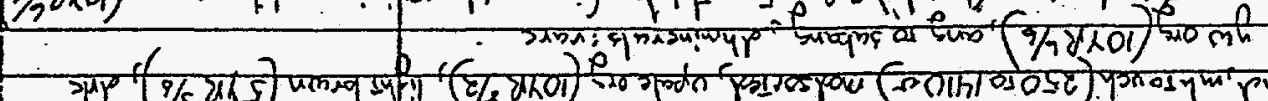

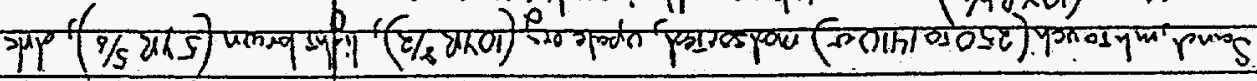

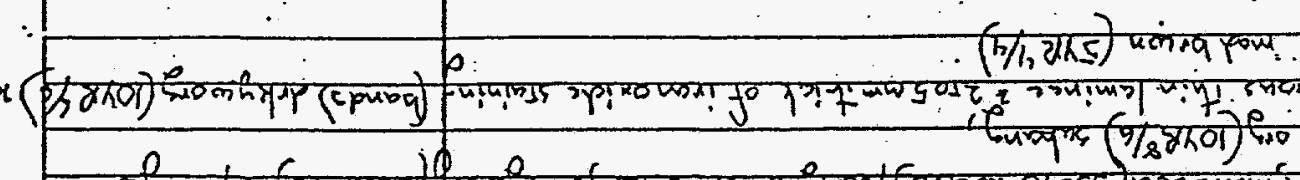

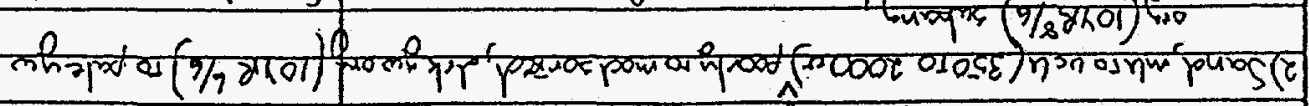

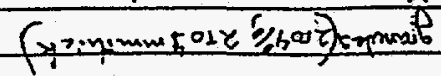

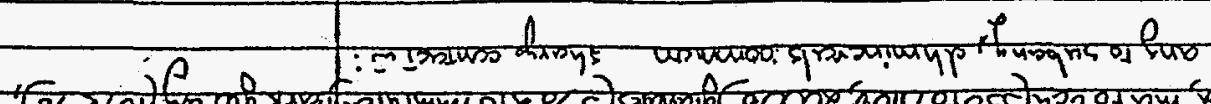

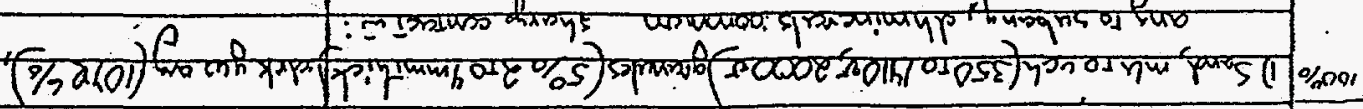

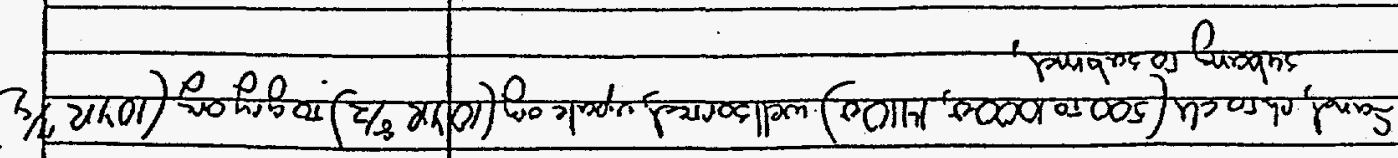
SIN3WWOS NOILI8OS 30 जาdW6S

SNIרוזם

\begin{tabular}{|c|c|}
\hline 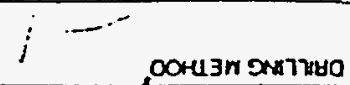 & surdmos \\
\hline yзmigo & s3um:Y000 ơs \\
\hline yosonesnos onkTivio & nกน \\
\hline
\end{tabular}

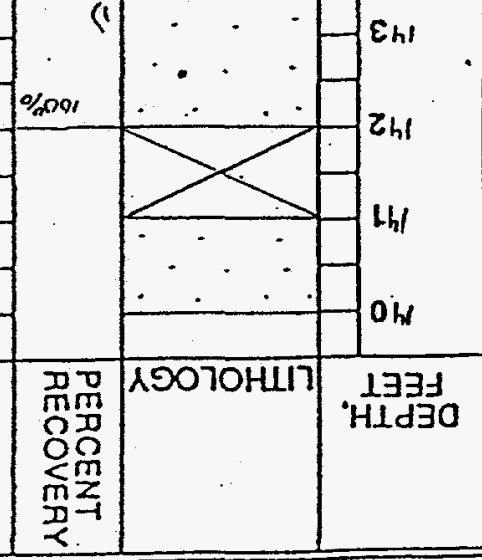




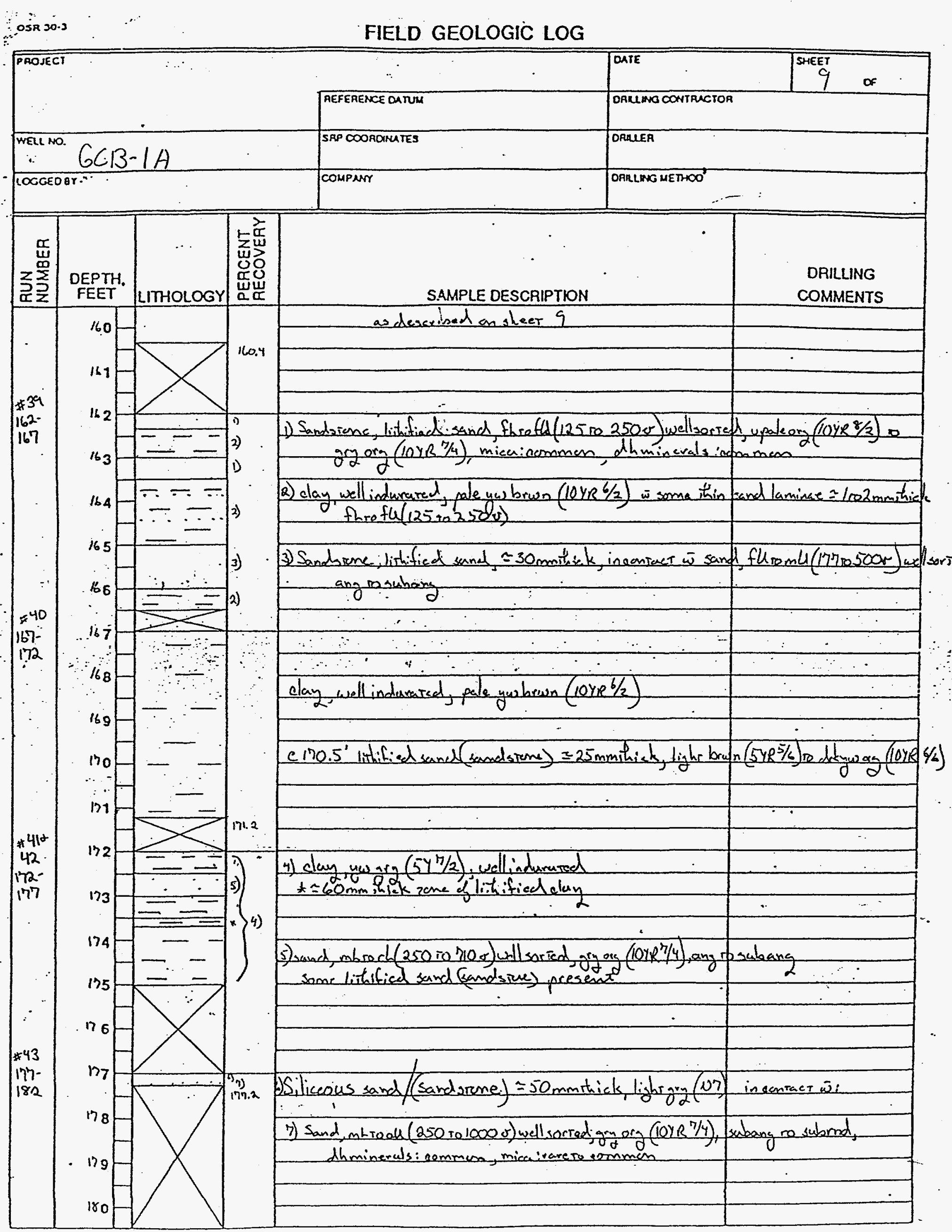




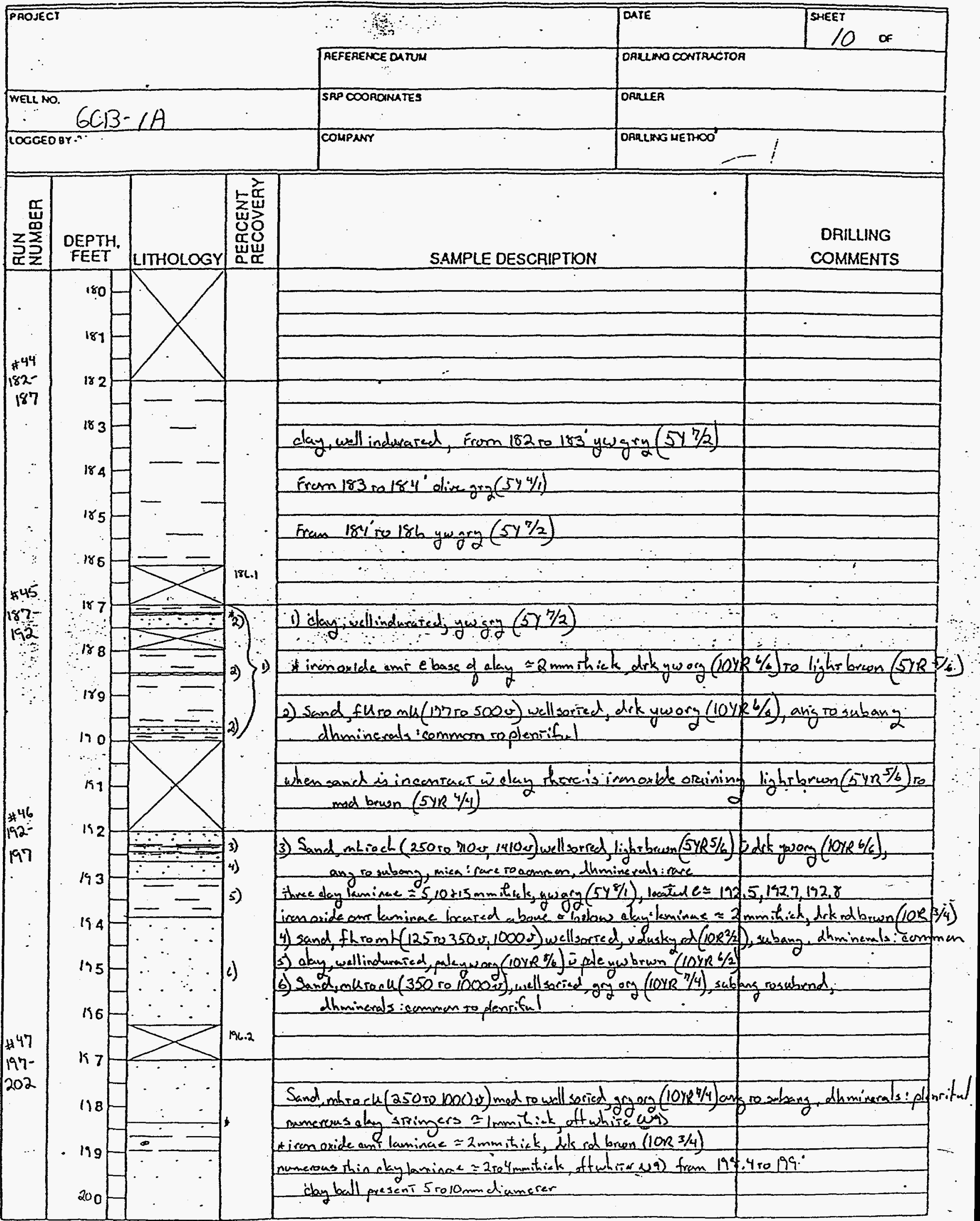


FIELD GEOLOGIC LOG

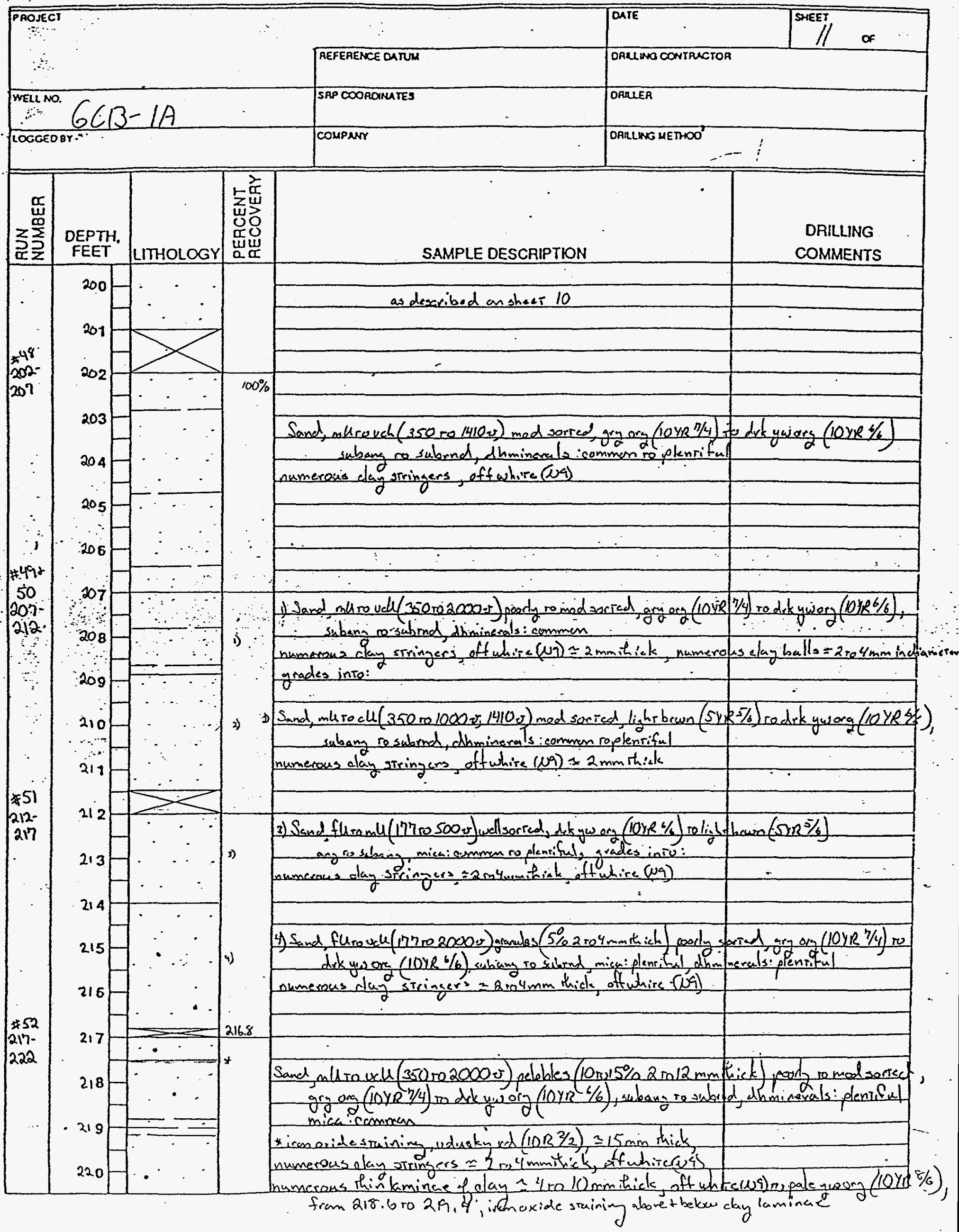


FIELD GEOLOGIC LOG

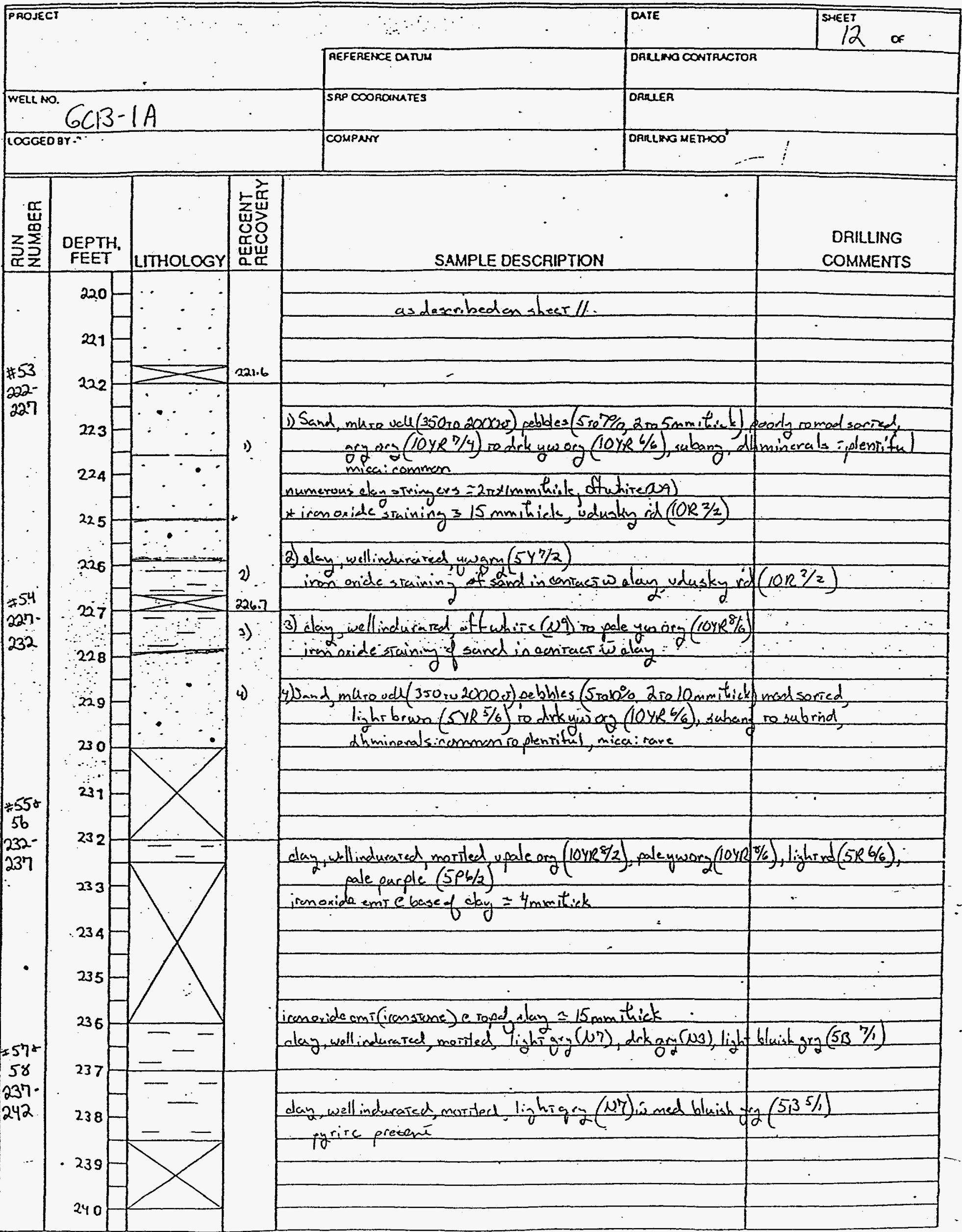


FIELD GEOLOGIC LOG

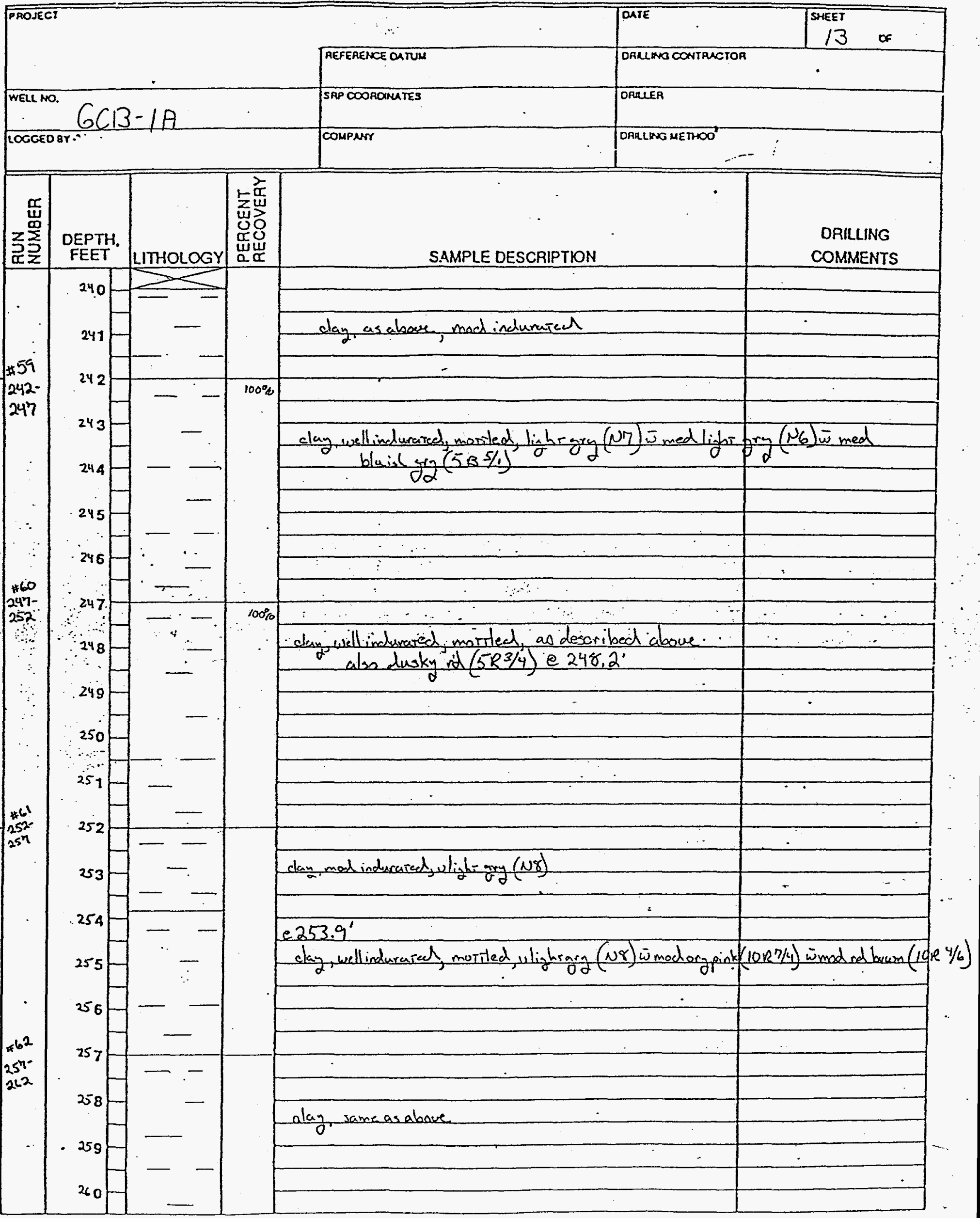







FIELD GEOLOGIC LOG

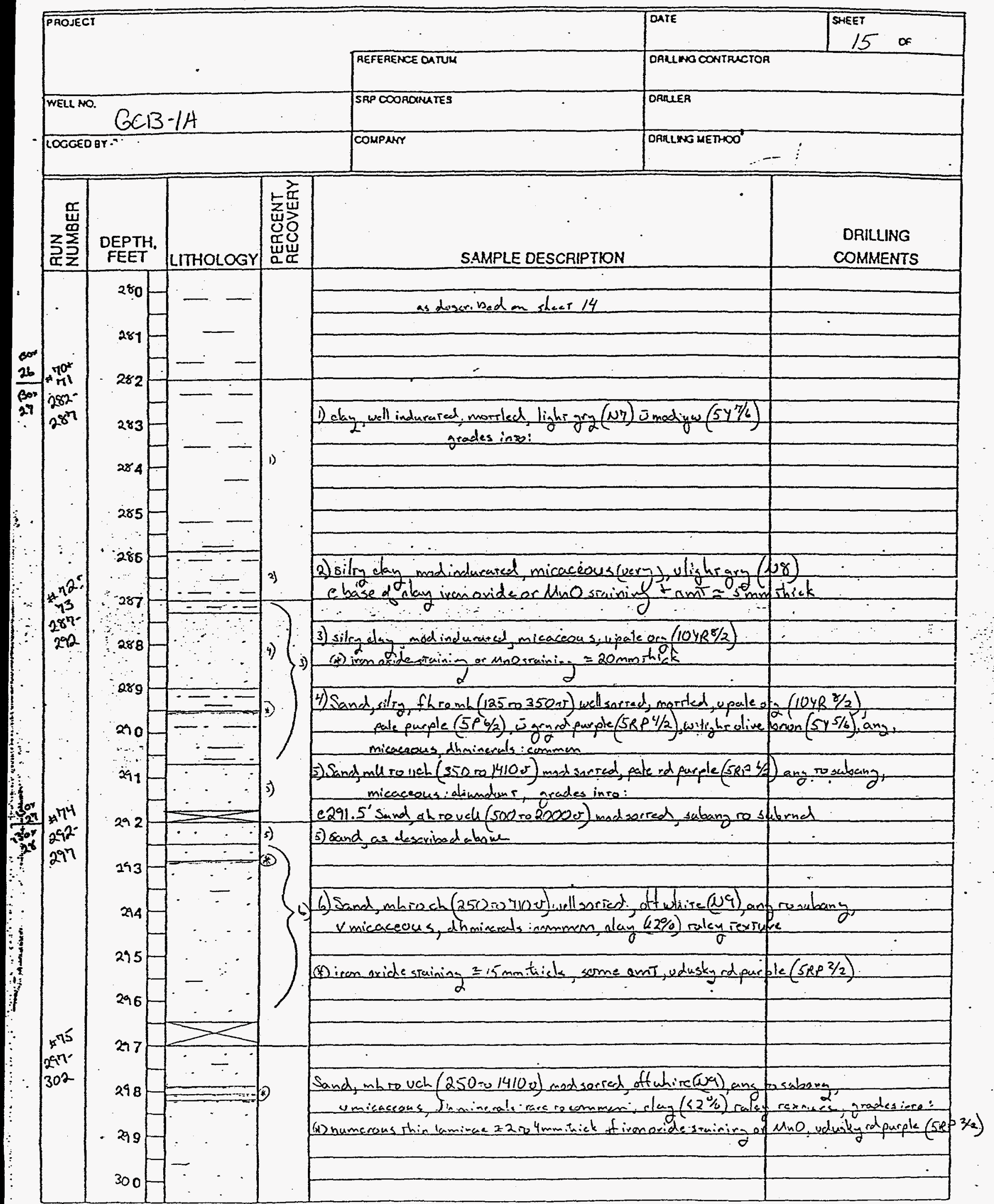




\section{FIELD GEOLOGIC LOG}

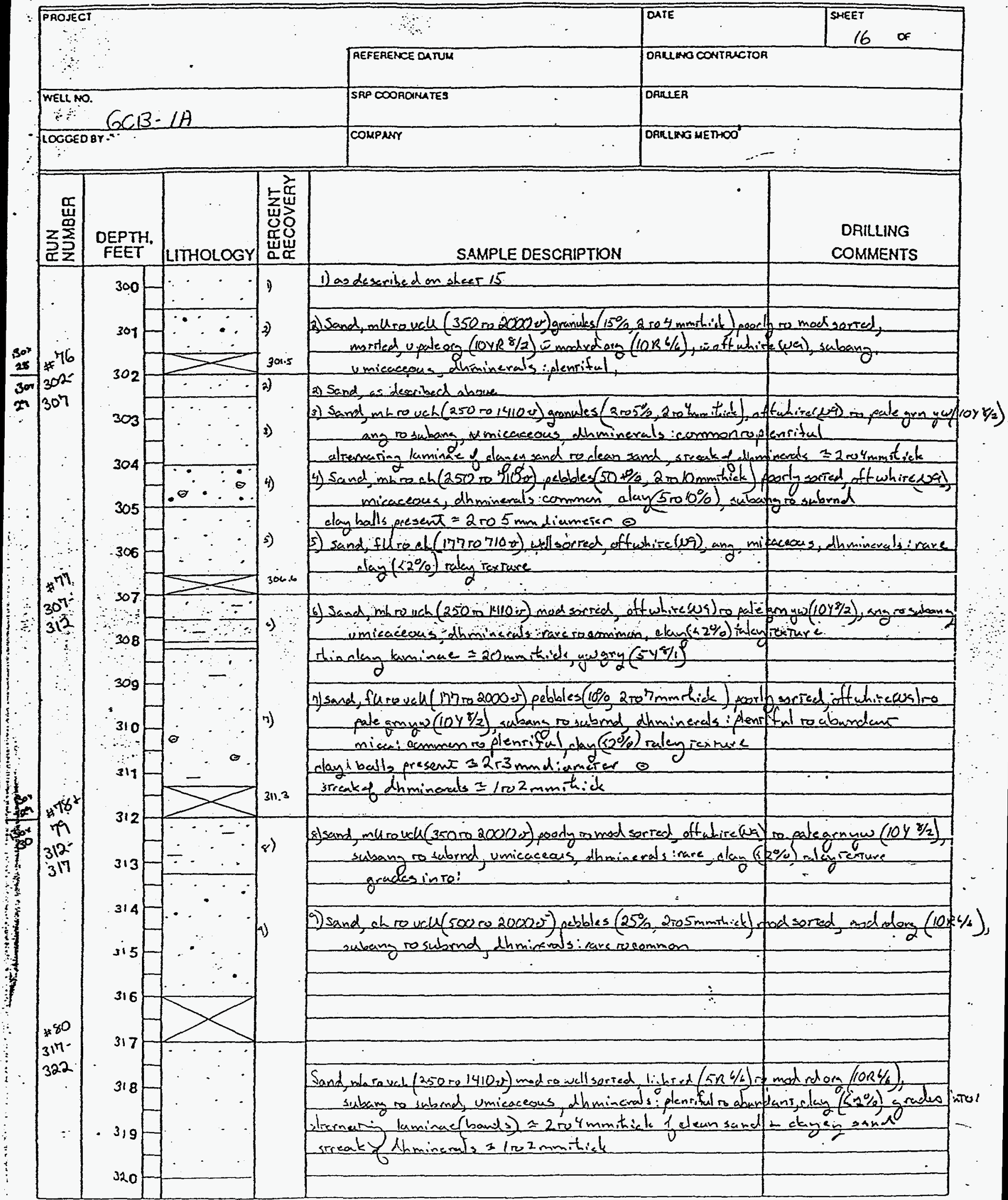


FIELD GEOLOGIC LOG

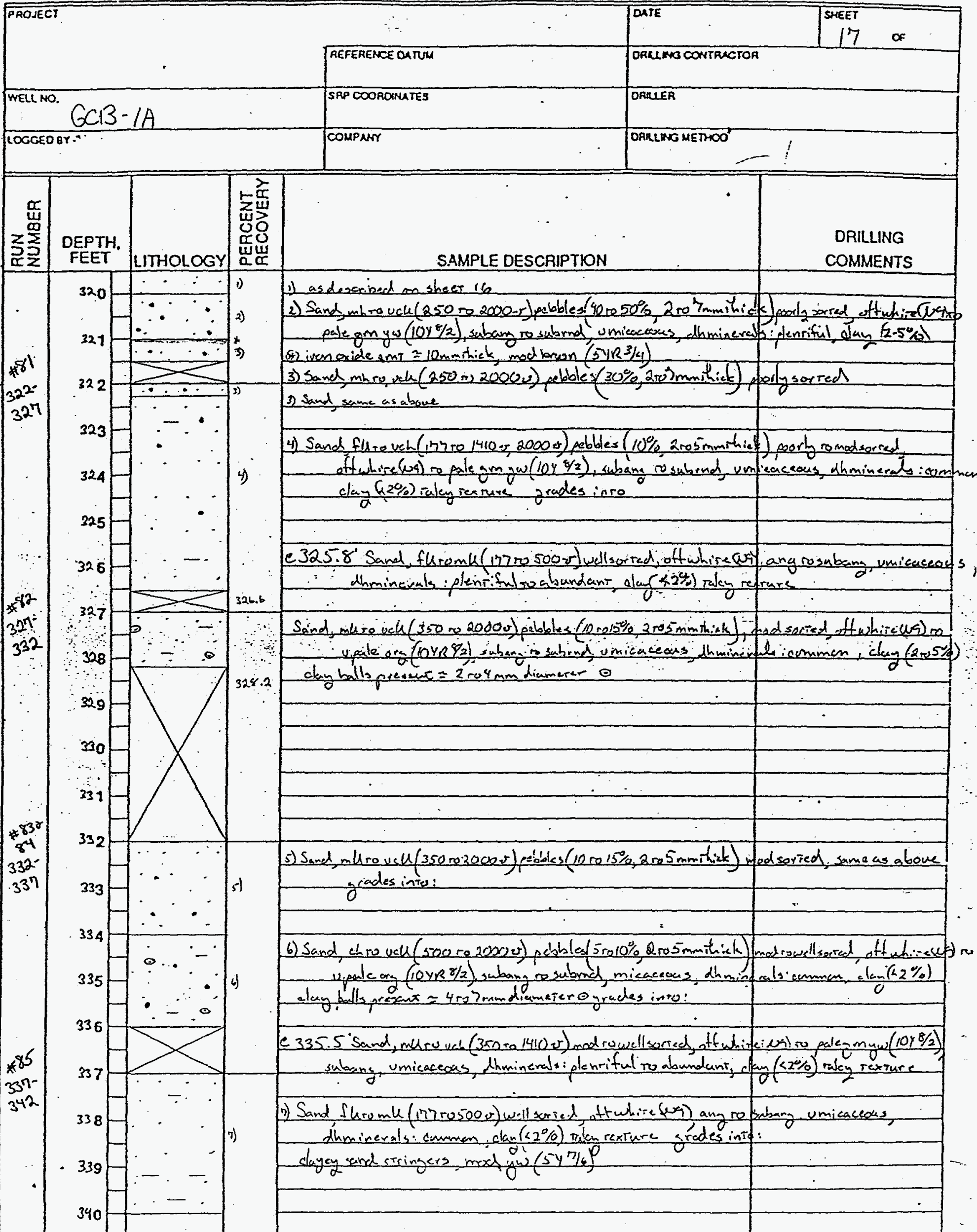




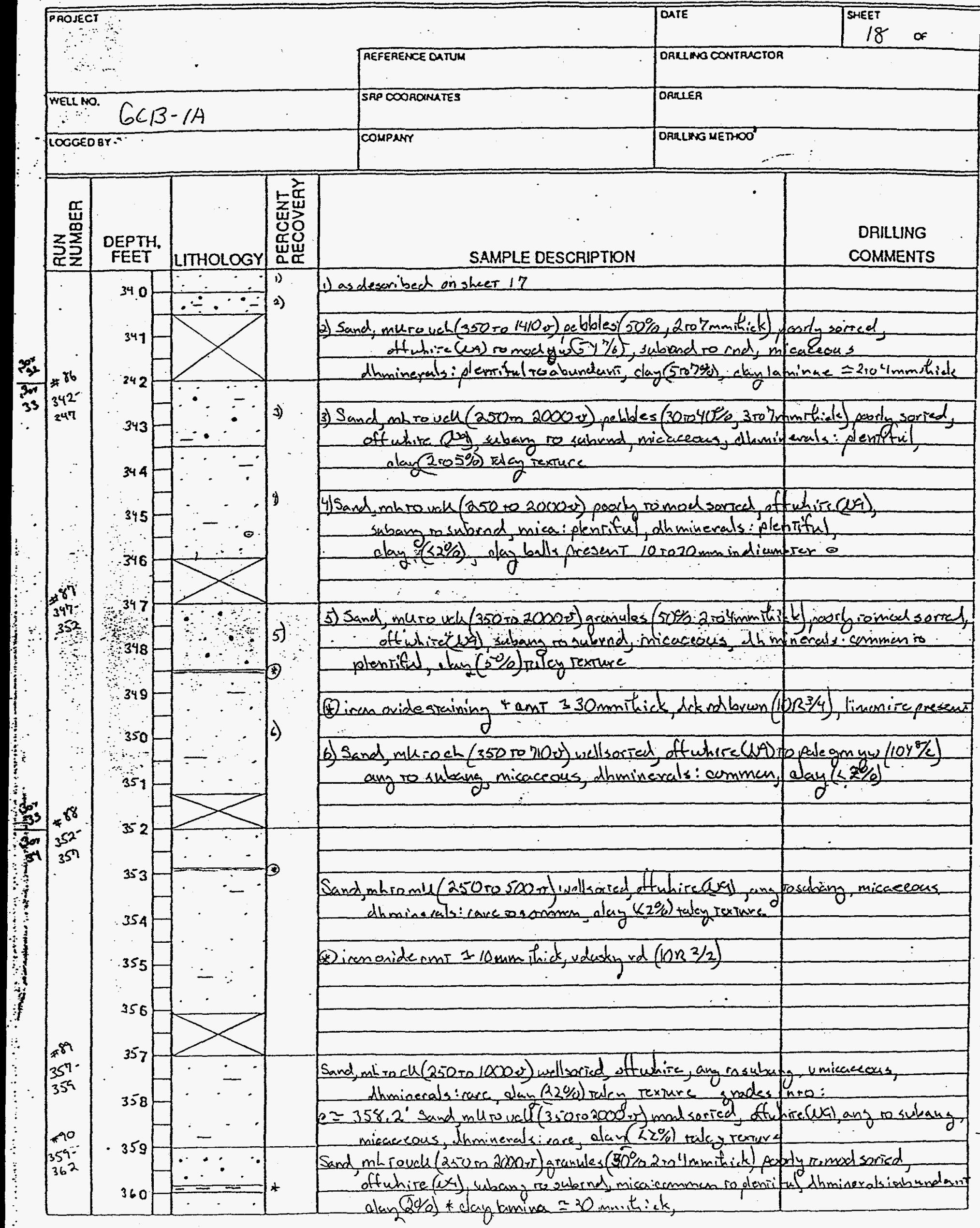


FIELD GEOLOGIC LOG

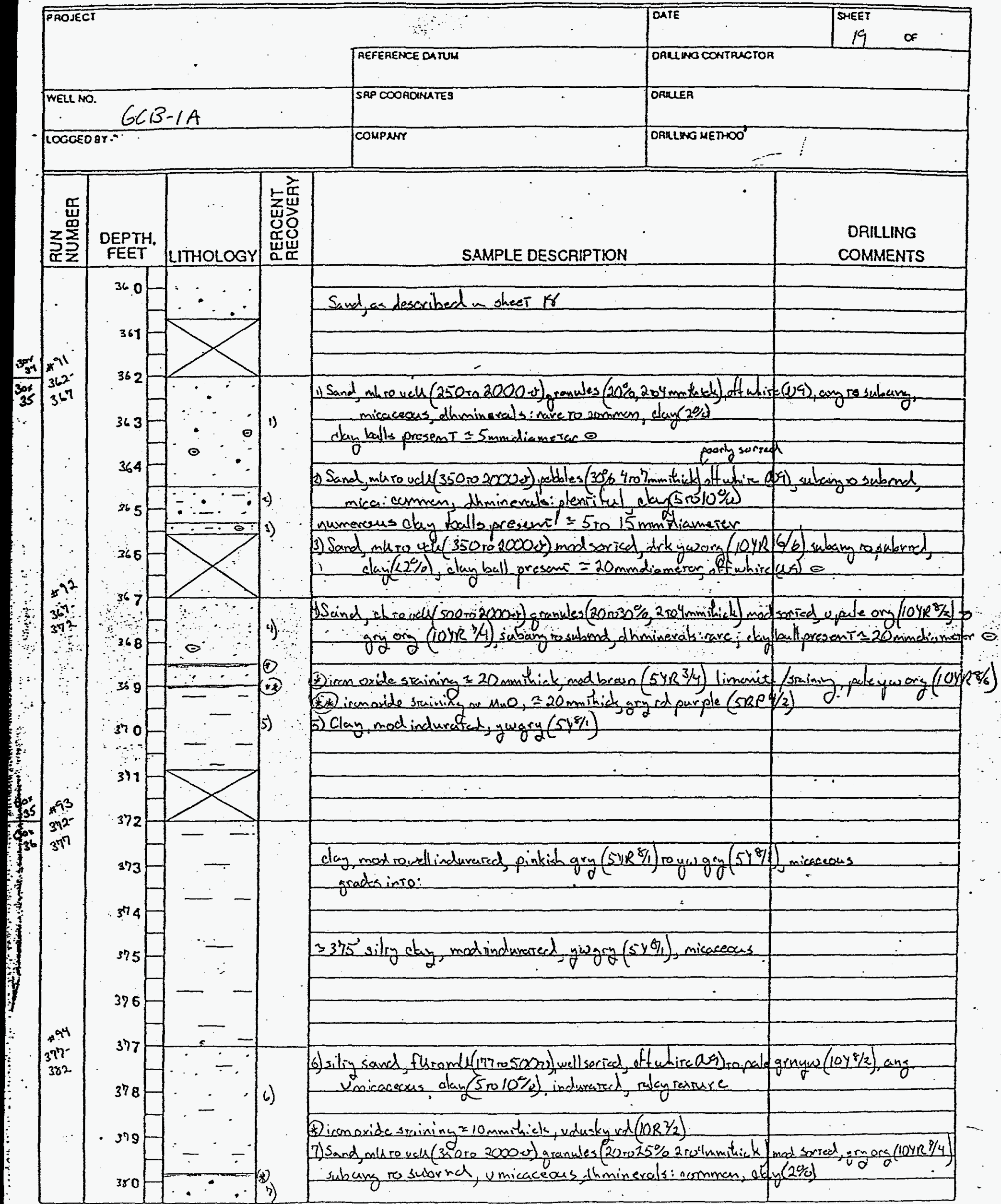




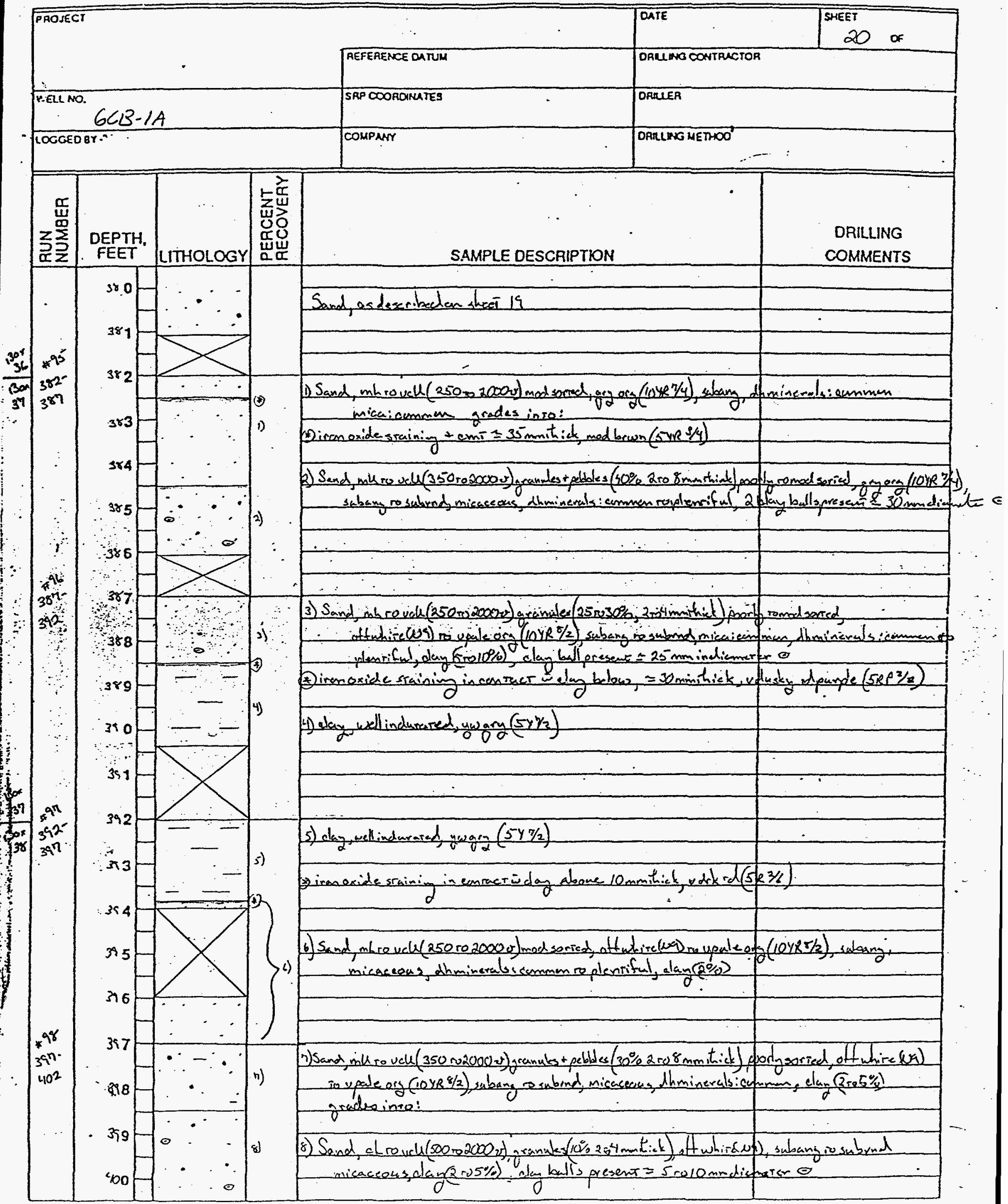


FIELD GEOLOGIC LOG

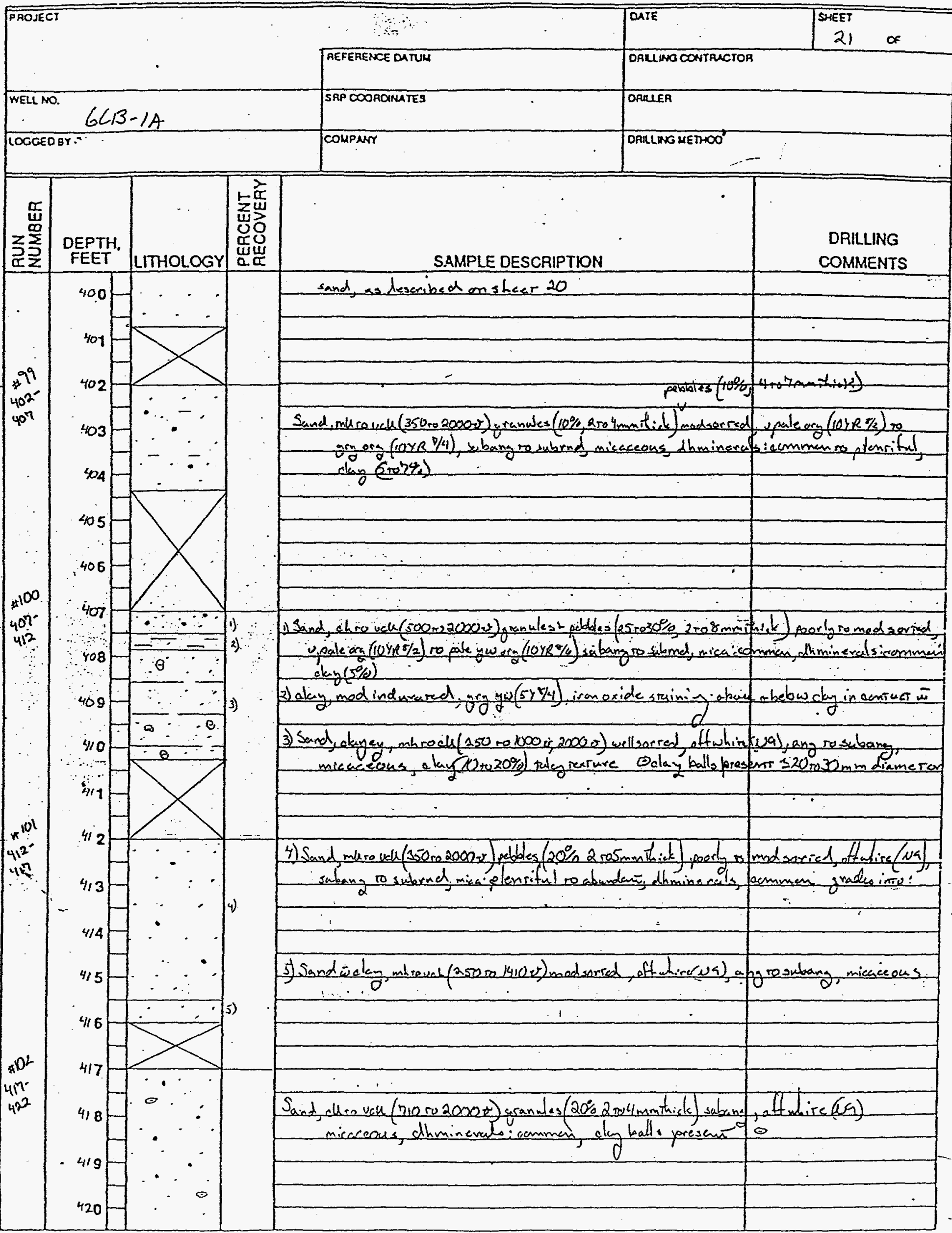


FIELD GEOLOGIC LOG

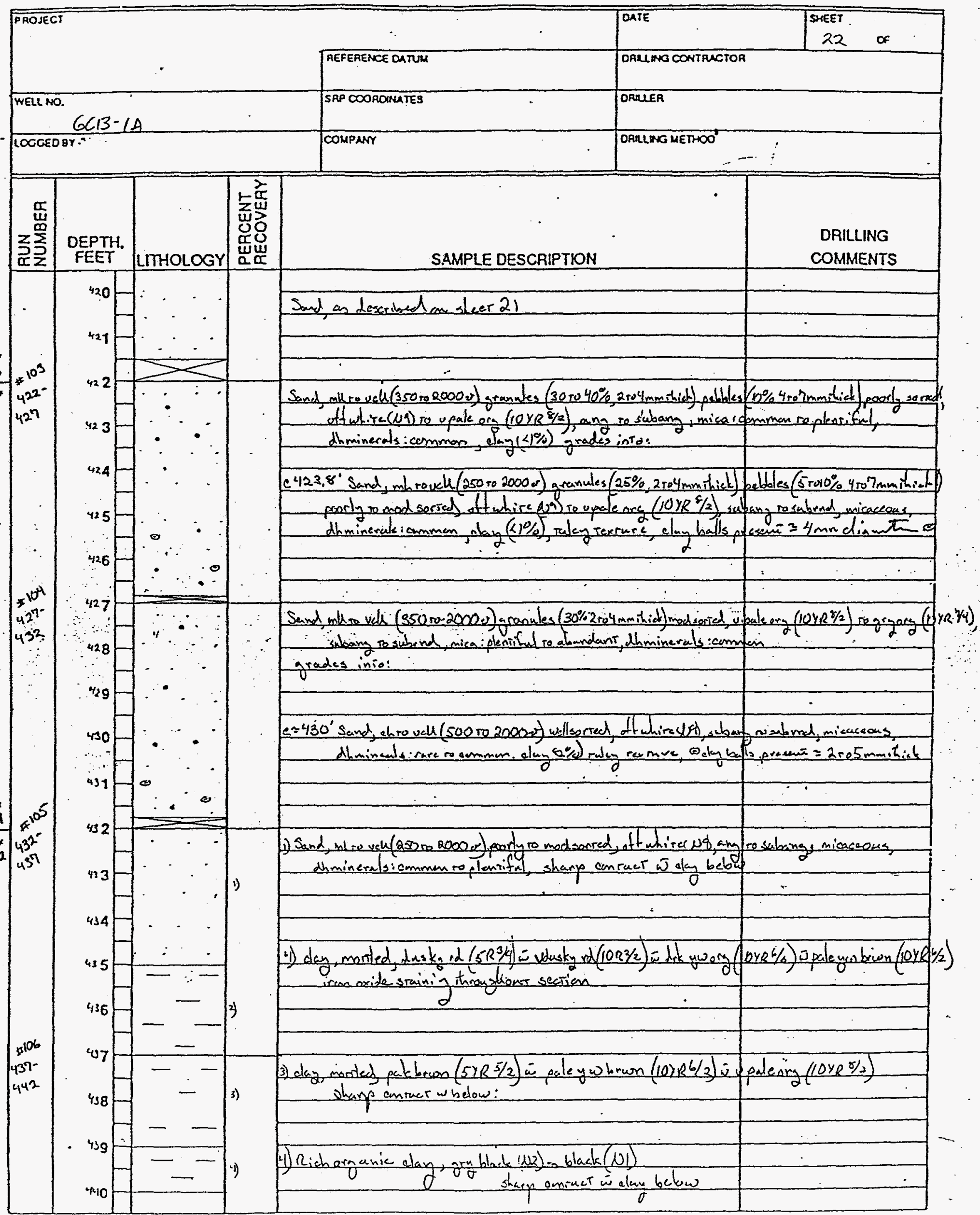


FIELD GEOLOGIC LOG

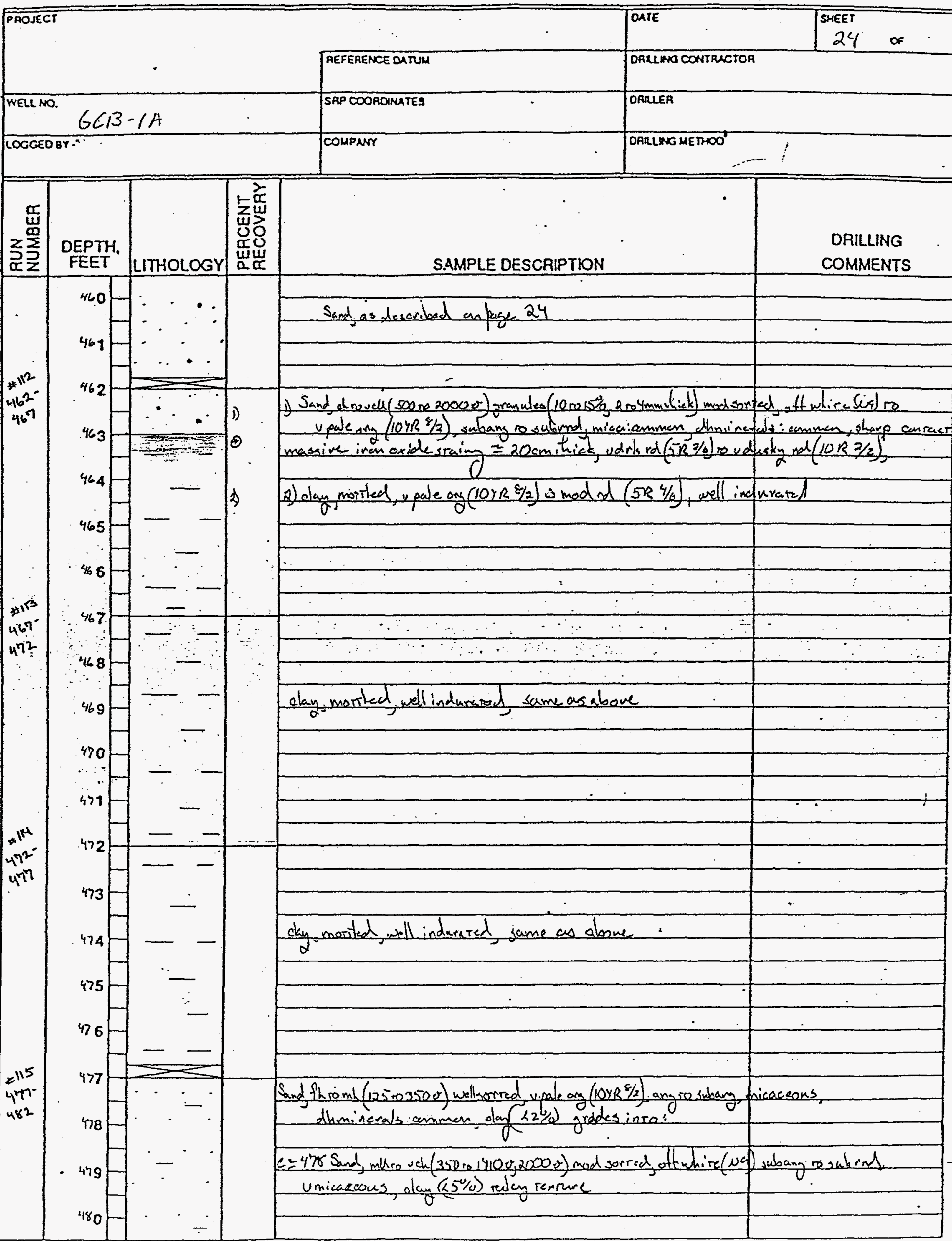




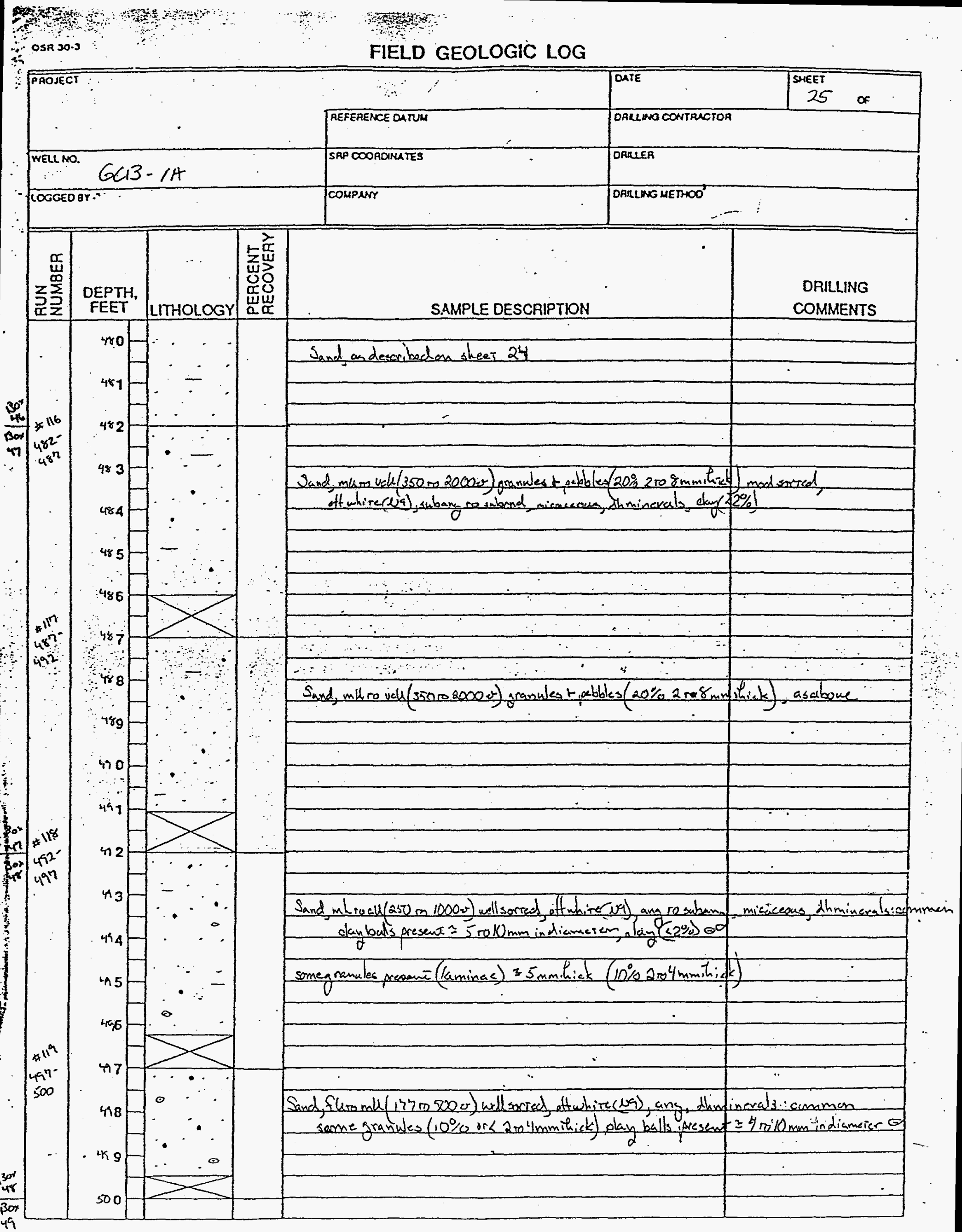







FIELD GEOLOGIC LOG

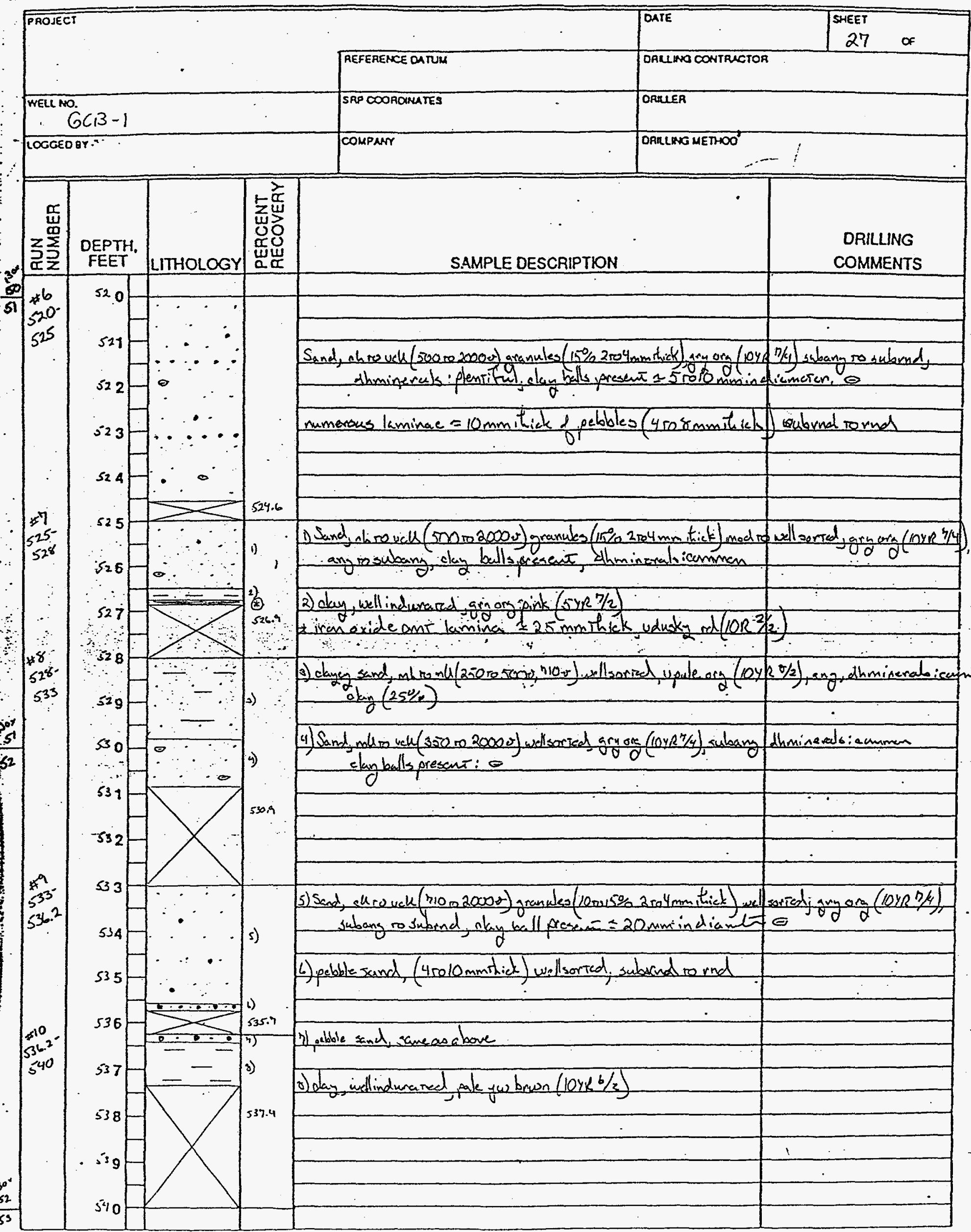




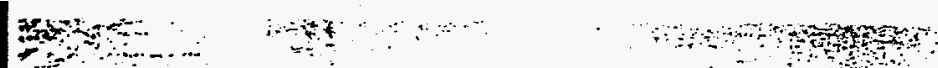

$\operatorname{OSR} 30.3$

FIELD GEOLOGIC LOG

\begin{tabular}{|c|c|c|c|}
\hline \multirow[t]{2}{*}{$\longdiv { \text { PROJECT } }$} & $\therefore$ & DATE & $\begin{array}{c}\text { SHEET } \\
29\end{array}$ \\
\hline & AEFERENCE CANUM & \multicolumn{2}{|c|}{ ORLLWO CONTRUCTOR } \\
\hline WELL NO. $G C B-1$ & SRP COOAROMUTES & \multicolumn{2}{|l|}{ OARLER } \\
\hline 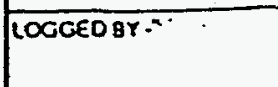 & COMPAN & \multicolumn{2}{|c|}{ DARLLWG METHOO } \\
\hline
\end{tabular}

\begin{tabular}{|c|c|c|c|}
\hline 䍃 & $\begin{array}{l}\text { DEPTH, } \\
\text { FEET }\end{array}$ & LITHOLOG & 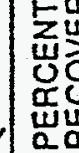 \\
\hline
\end{tabular}

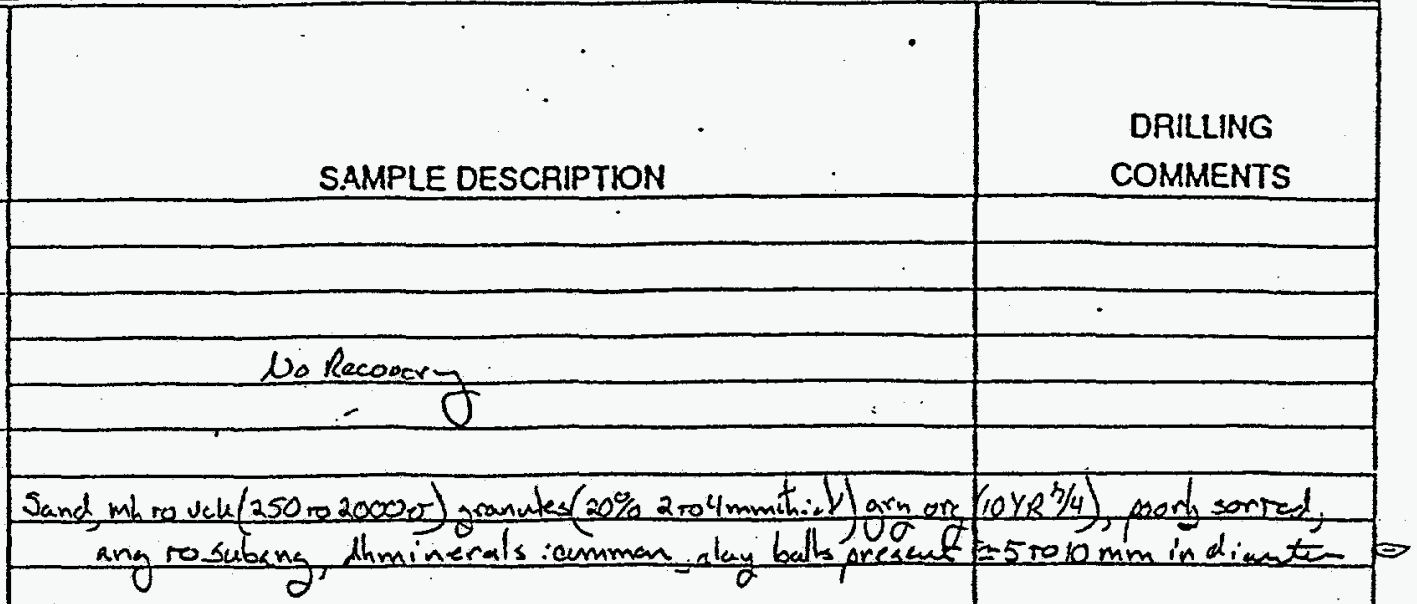

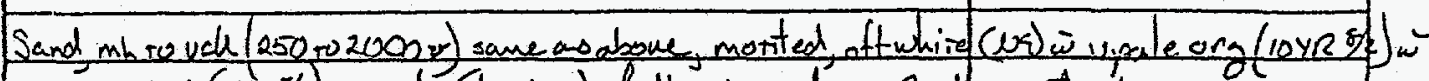

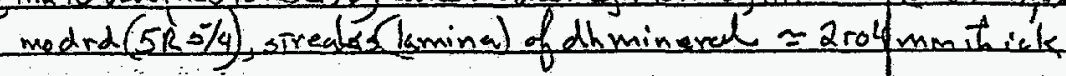

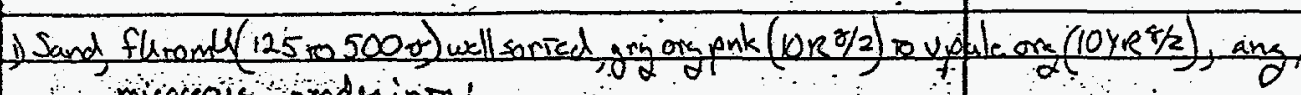
micacious grades into:

* iron oxide or $\overline{\mu n O}$ ont $\cong 5$ mmitick ...

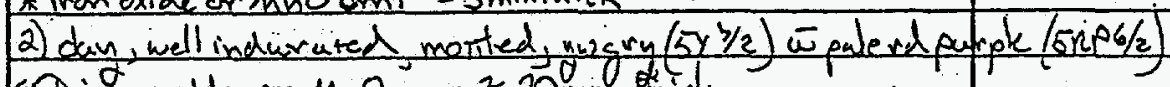

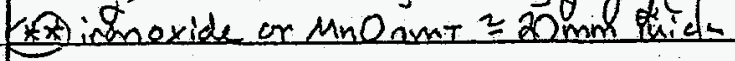

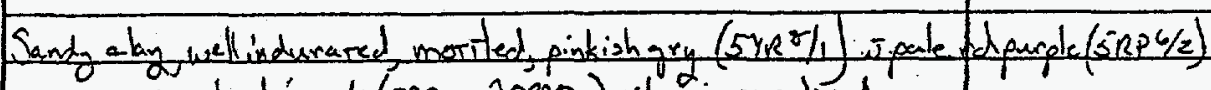
$\sin \div-1$ sand, ahio vele (500 ro 20e00s) sibang

Sandy alan well indurared same as above

Sands alan, ellindurared, sume as above grades inro:

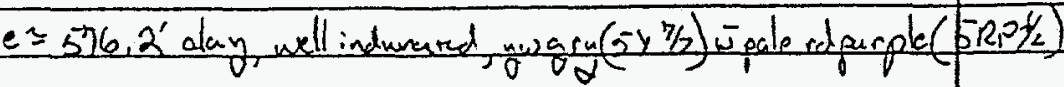

doy, wellindurared, same us above 


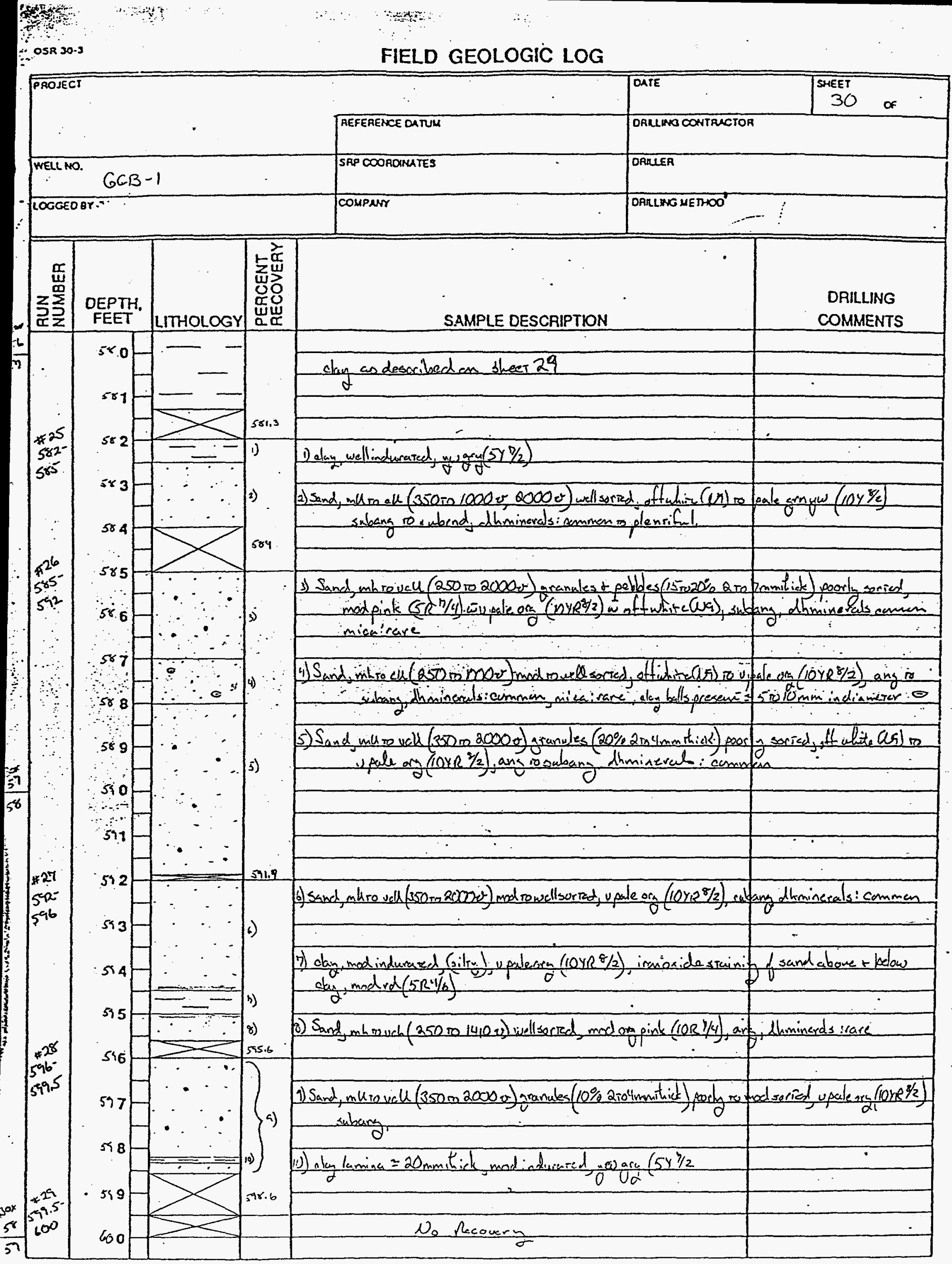


FIELD GEOLOGIC LOG

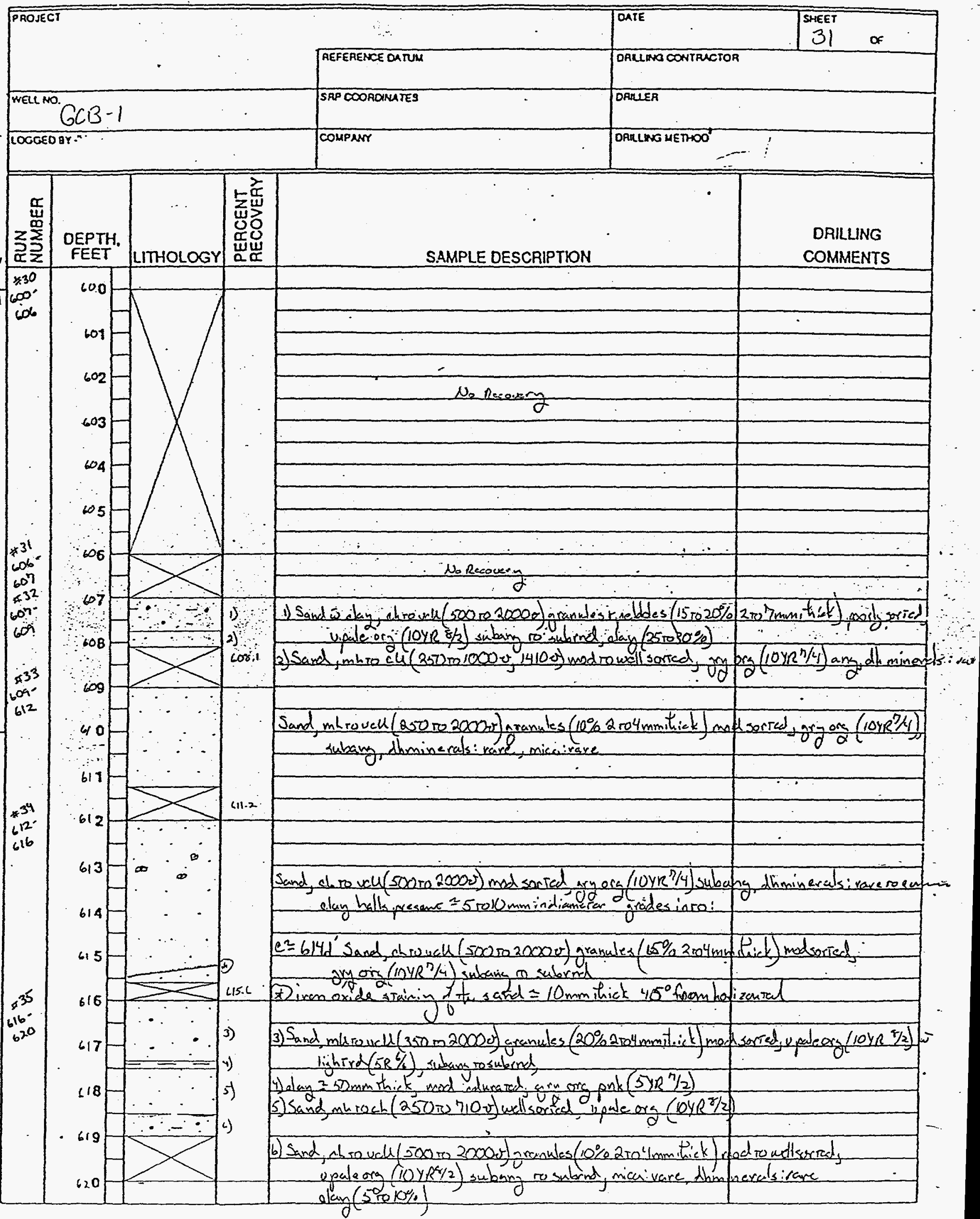


FIELD GEOLOGIC LOG

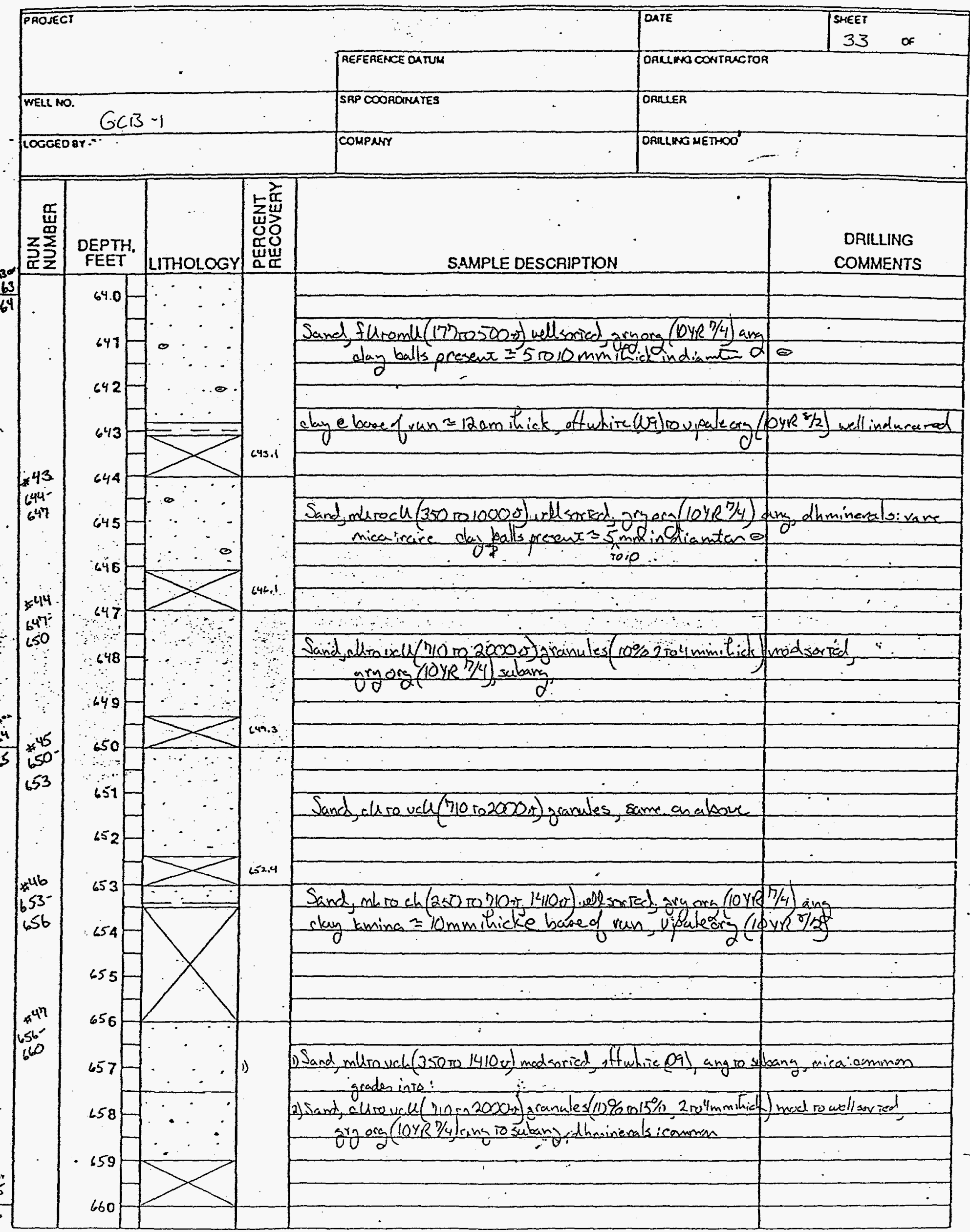




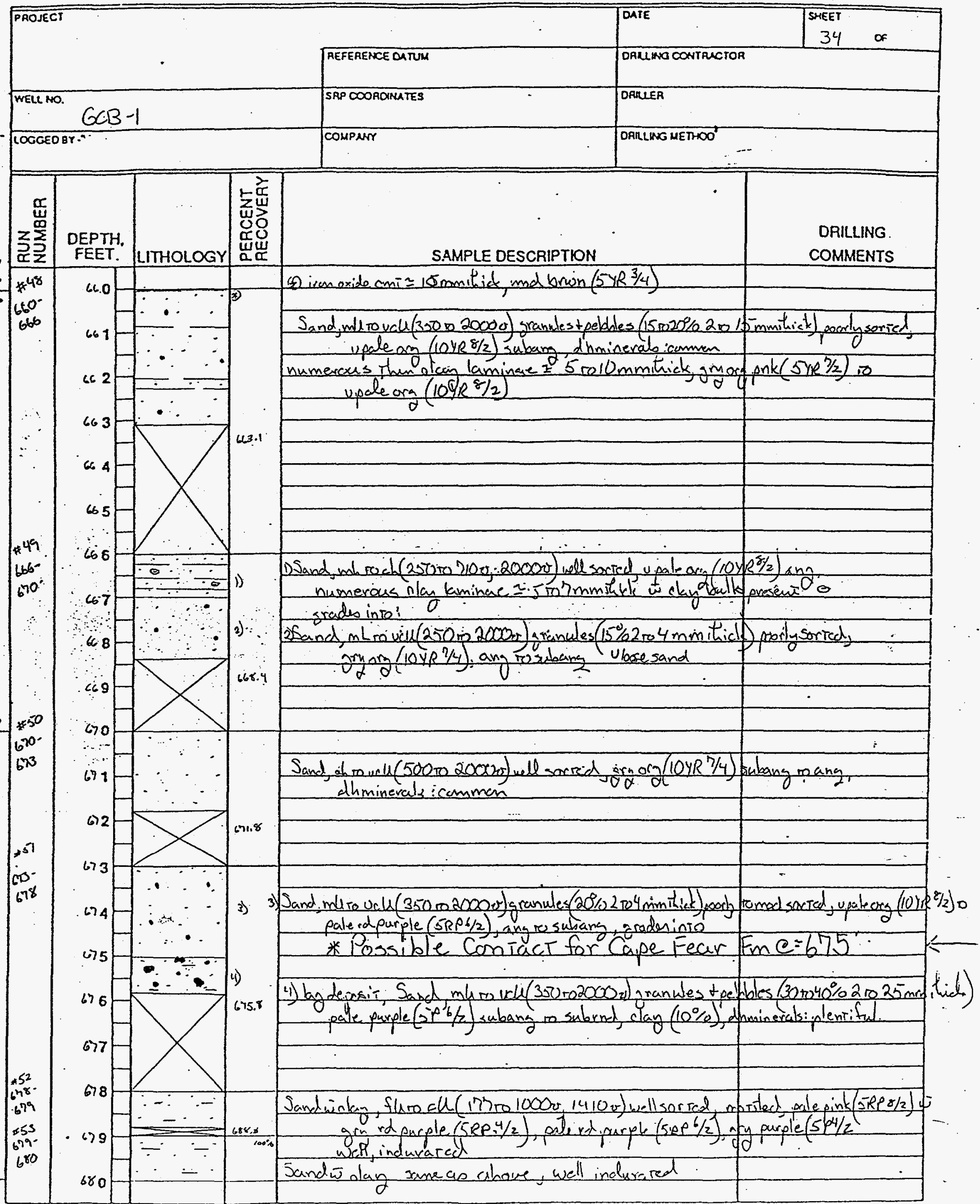


FIELD GEOLOGIC LOG

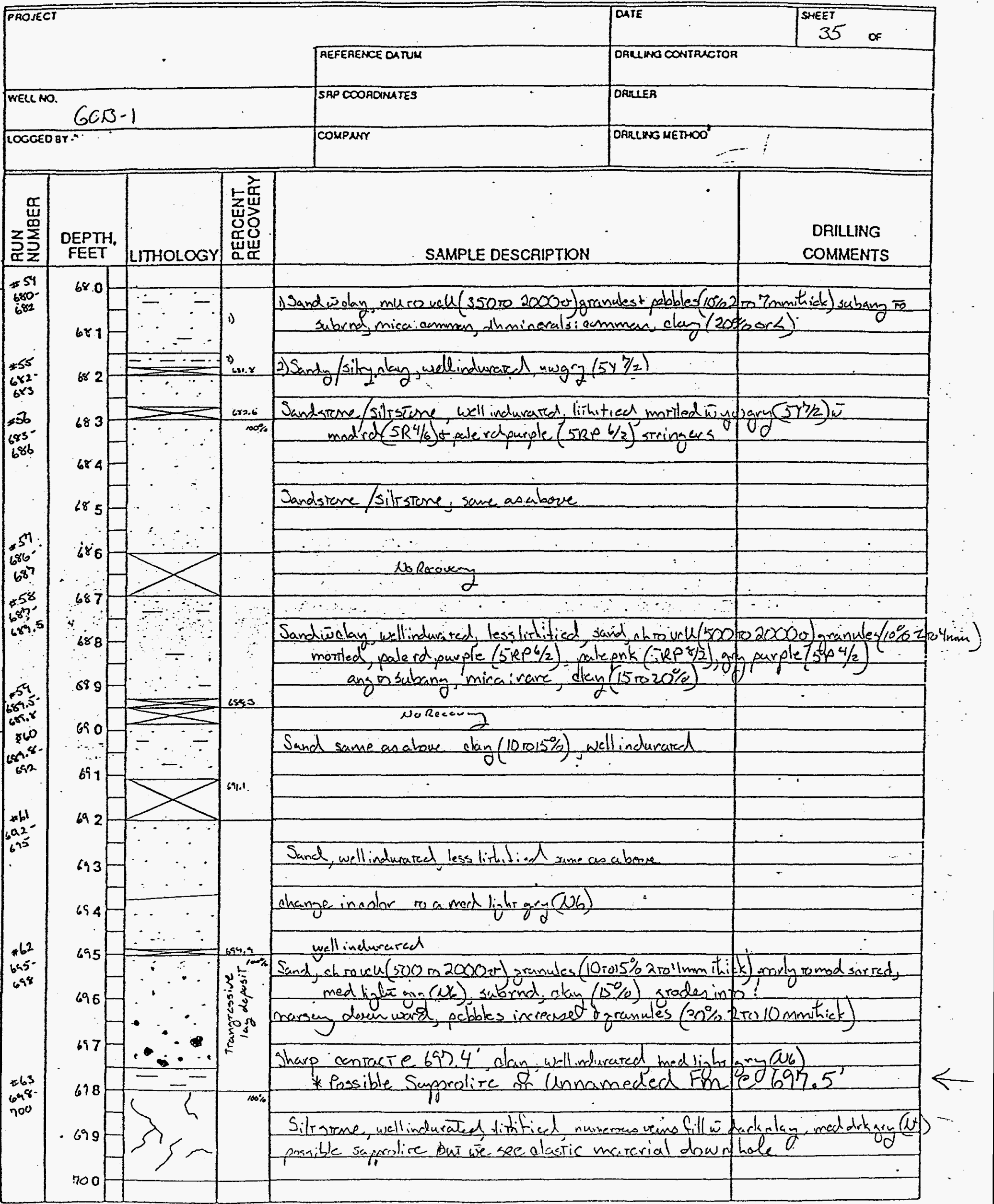


FIELD GEOLOGIC LOG

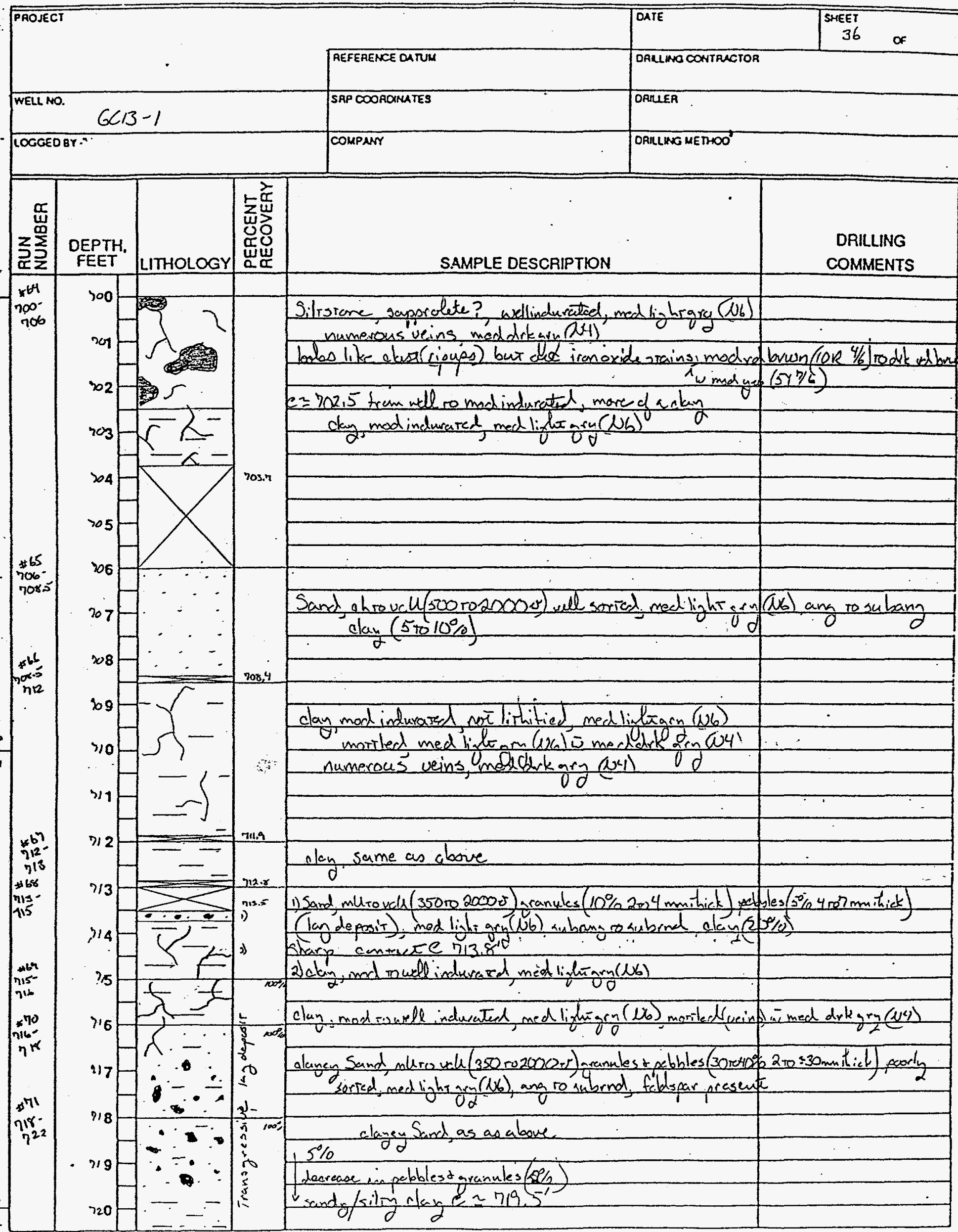


FIELD GEOLOGIC LOG

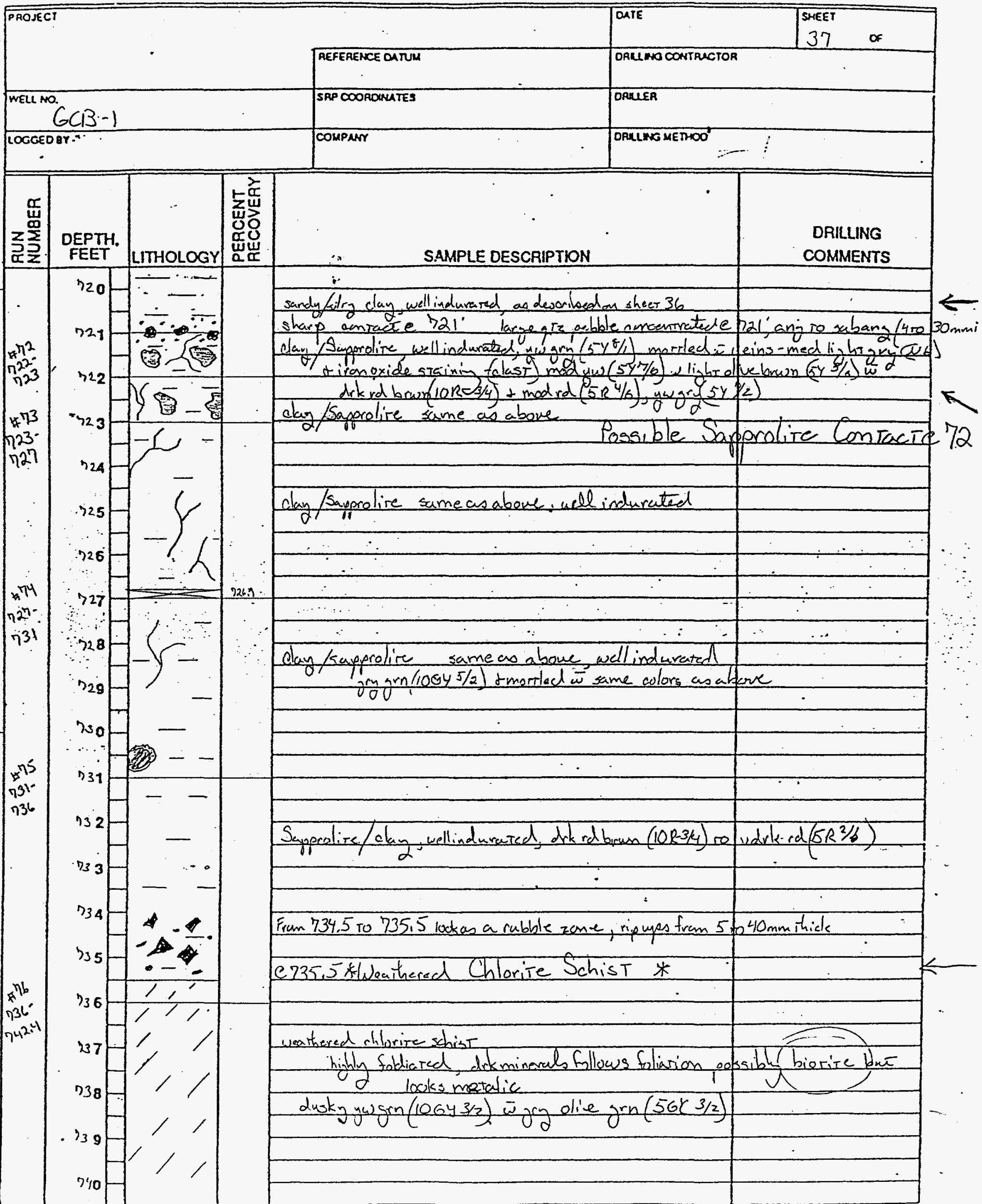


FIELD GEOLOGIC LOG

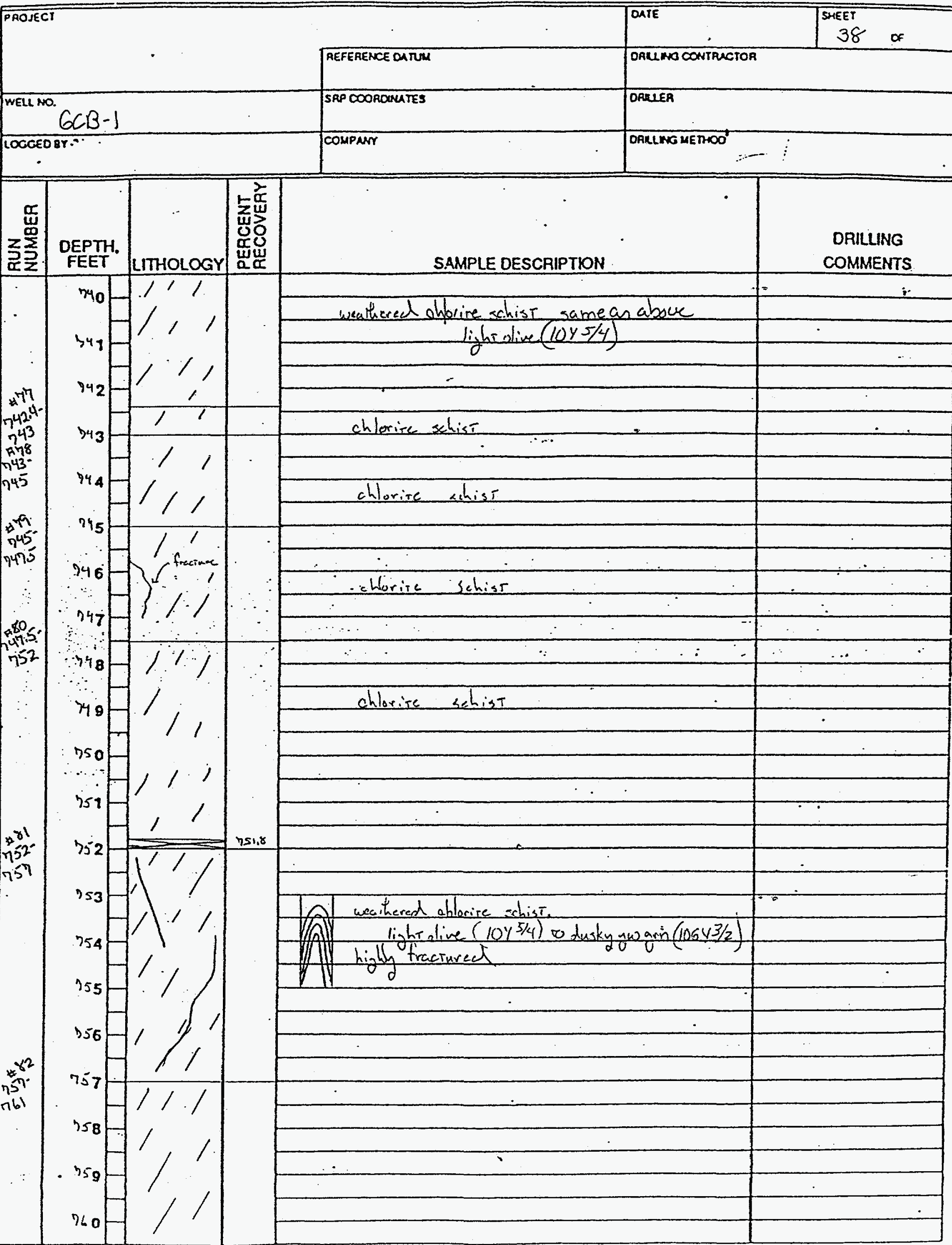


FIELD GEOLOGIC LOG

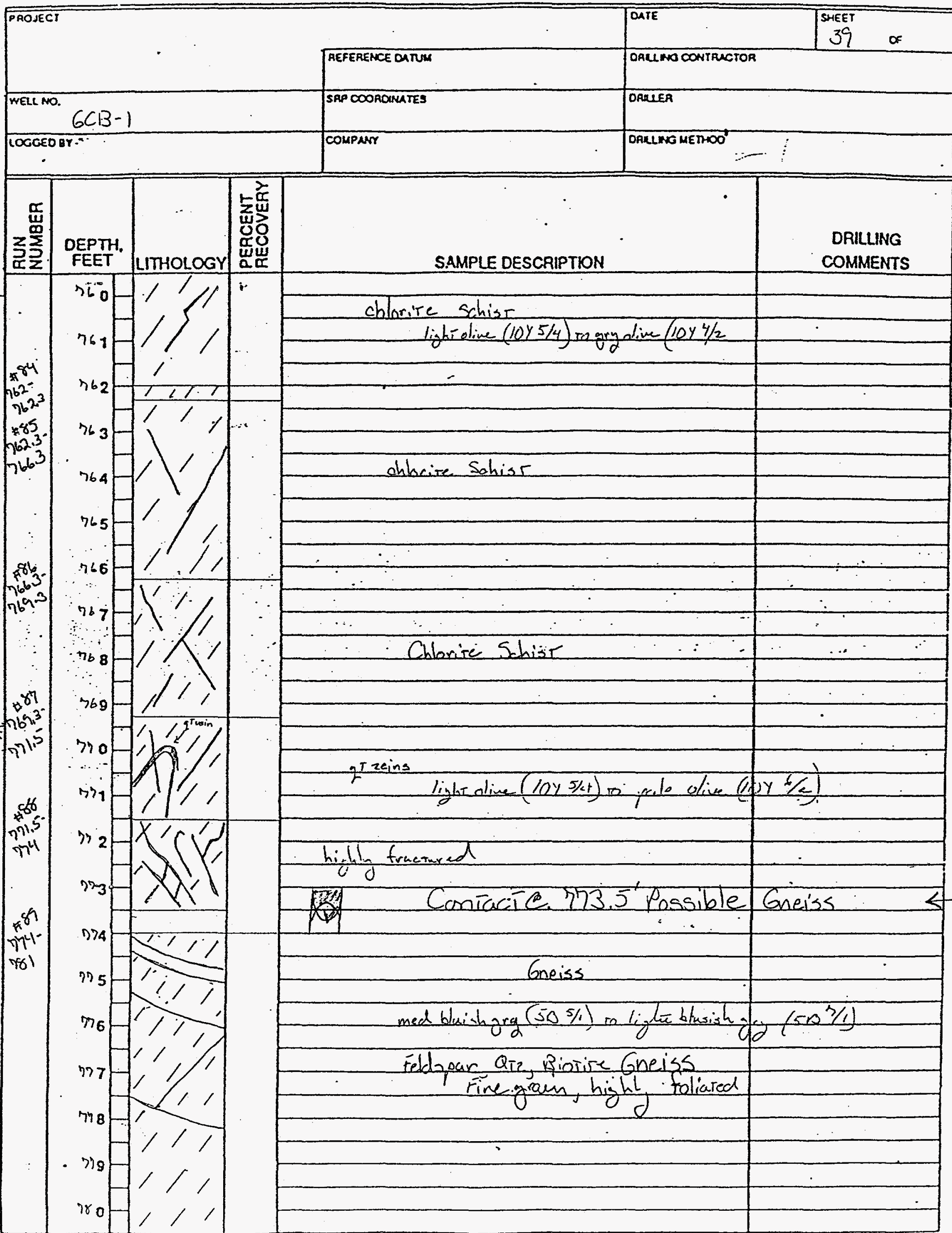


FIELD GEOLOGIC LOG

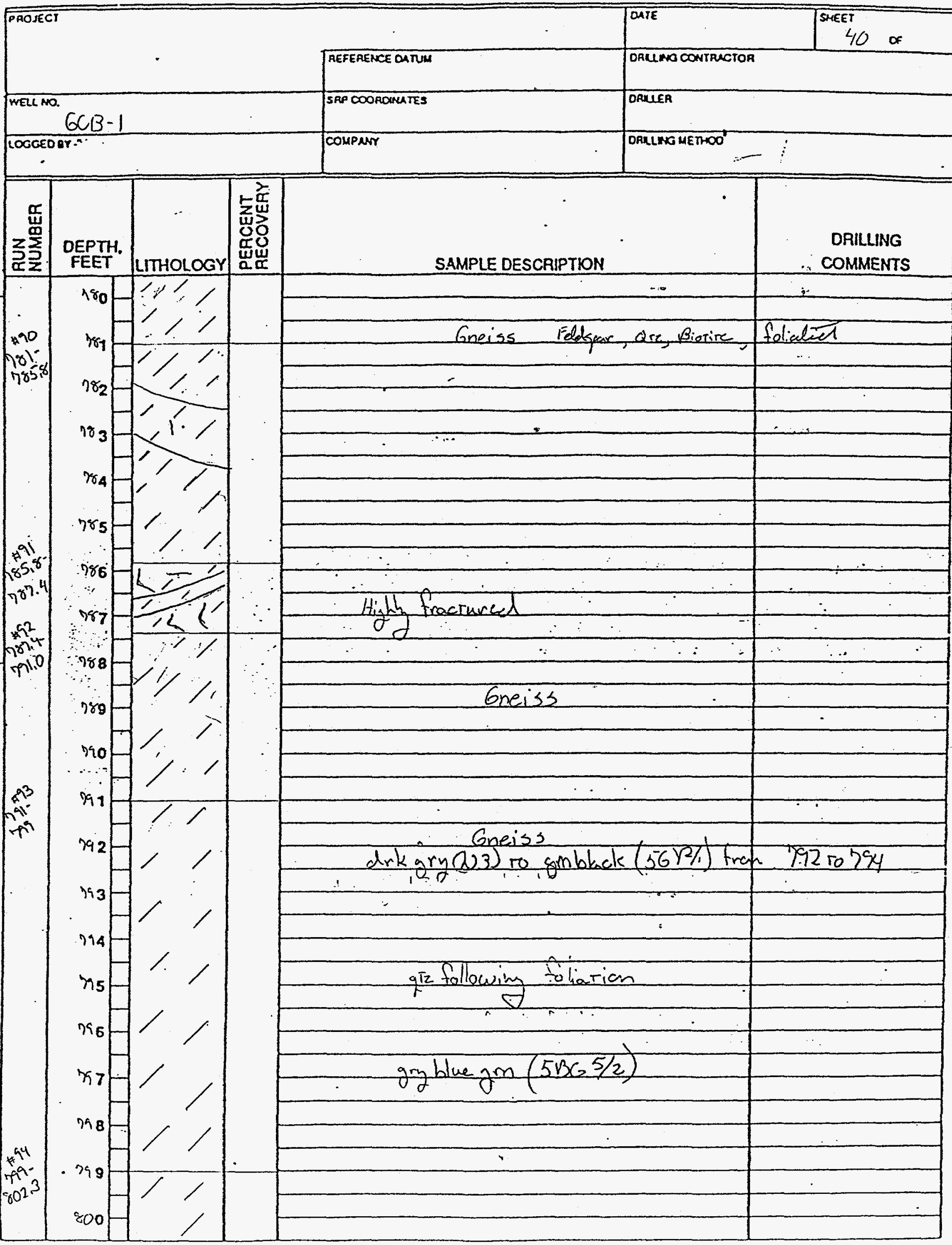


FIELD GEOLOGIC LOG

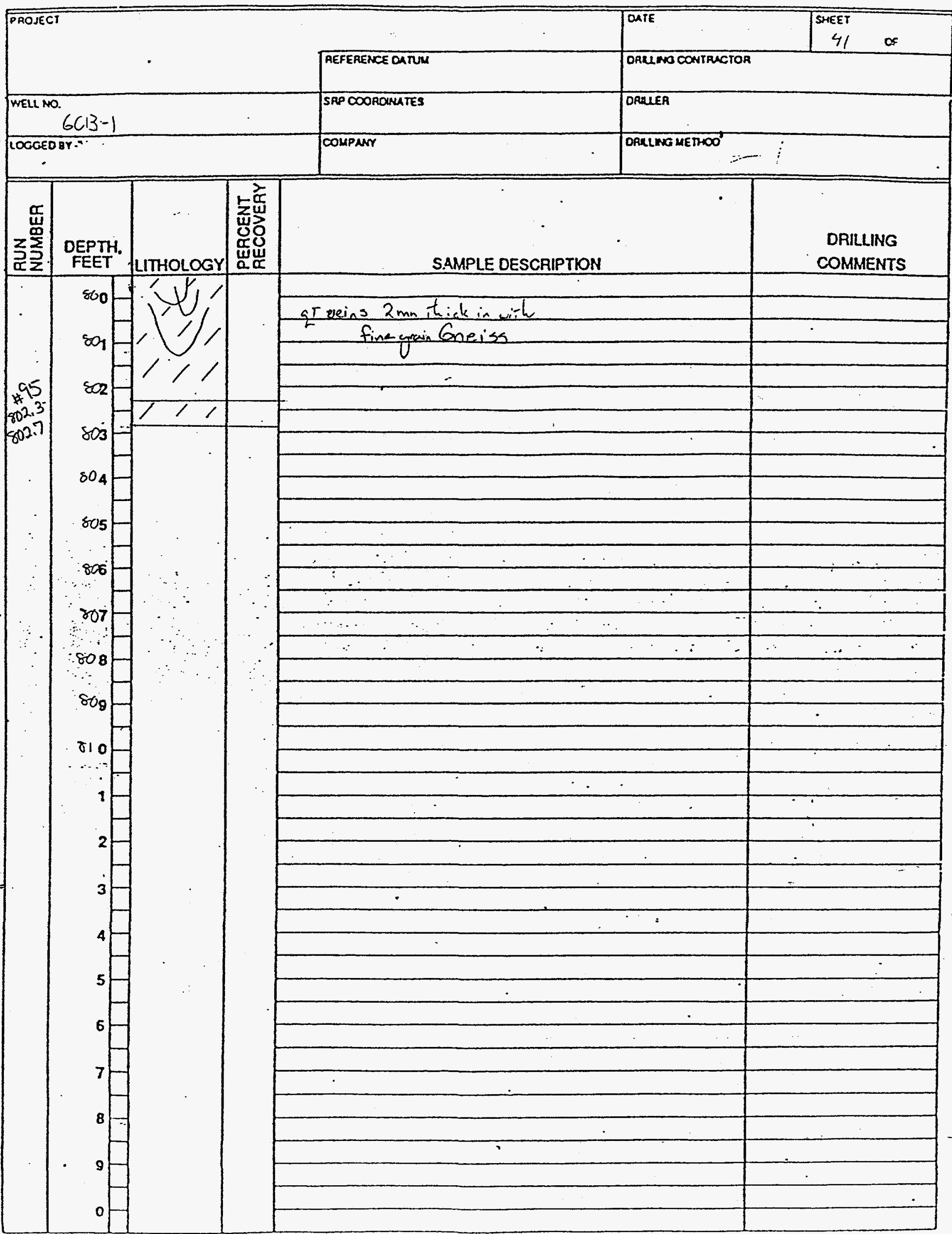


Core Hole: \# GCB-2

6-4-96

Rotary down to $12 \mathrm{ft}$.

$\begin{array}{ll}\text { Run 1 } & 12^{\prime}-17^{\prime} \\ \text { Run 2 } & 17-20^{\prime} \\ \text { Run 3 } & 20-25^{\prime} \\ \text { Run 4 } & 25-30^{\prime} \\ \text { Run 5 } & 30-33^{\prime} \\ \text { Run 6 } & 33-40 \\ \text { Run 7 } & 40-45^{\prime} \\ \text { Run 8 } & 45-51 \\ \text { Run 9 } & 51-55 \\ \text { Run 10 } & 55-60\end{array}$

Recovered:

$4^{\prime}$ 13-17'

' $19-20^{\prime}$

$4^{\prime} \quad 21-25^{\prime}$

3.8' 26.2-30'

4" $32^{\prime \prime} 8^{\prime \prime}-33^{\prime}$

no recovery

no recovery

9" $50^{\prime \prime} 3^{\prime \prime}-51^{\prime}$

no recovery

no recovery

6-6-96

mud viscosity: $51 \mathrm{sec}$

Run 11 60-65'

Run 12 65-71'

$4^{\prime} 5^{\prime \prime} \quad 60^{\prime} 7^{\prime \prime}-65^{\prime}$

Run 13 71-75'

6' $\quad 100 \%$

$2^{\prime} 8^{\prime \prime} \quad 72^{\prime} 4^{\prime \prime}-75^{\prime}$

Run 14 75-76'

$3^{\prime}$. 75'9"-76

$2^{\prime} 7^{\prime \prime} \quad 77^{\prime} 5^{\prime \prime}-80^{\prime}$

Run 15 76-80'

Run 16 80-85'

$4^{\prime} 8^{\prime \prime} \quad 80^{\prime} 4^{\prime \prime}-85^{\prime}$

Run 17 85-91'

Run 18 91-95'

4'6" 86' 6"-91'

$100 \%$

Lost 96'1"-96'8"

Run 19 95-97'

Run $20 \quad 97-100$

Run $21 \quad 100-105$

$2^{\prime}$ 98-100'

no recovery

6-7-96

Run 22 105-109

Run 23 109-112

Run 24 112-114

Run 25 114-117

Run 26 117-120

Run 27 120-123

Run 28 123-127

Run 29 127-131

Run 30 131-135'

mud viscosity: $63 \mathrm{sec}$

Run 31 135-141

Run 32 141-144

Run 33 144-147

Run 34 147-152

$\begin{array}{ll}3^{\prime} & 106-109^{\prime} \\ 10^{\prime \prime} & 109-109^{\prime} 10^{\prime \prime} \\ 9^{\prime \prime} & 113^{\prime} 3^{\prime \prime}-114^{\prime} \\ \text { no recovery } \\ \text { no recovery } \\ 1^{\prime} 3^{\prime \prime} & 121^{\prime} 9^{\prime \prime}-123^{\prime} \\ 1^{\prime} 8^{\prime \prime} & 125^{\prime} 4^{\prime \prime}-127^{\prime} \\ 4^{\prime} & 100 \% \\ 1^{\prime} 7^{\prime \prime} & 131-132^{\prime} 7^{\prime \prime} \\ & \\ 100 \% & \\ 2^{\prime} 5^{\prime \prime} & 141-143^{\prime} 5^{\prime \prime} \\ 2^{\prime} 9^{\prime \prime} & 1443^{\prime \prime}-147^{\prime} \\ 9^{\prime \prime} & 147-147^{\prime \prime} 9^{\prime \prime}\end{array}$




\begin{tabular}{|c|c|c|c|c|}
\hline Run 35 & $152-153$ & $10^{\prime \prime}$ & $152-152^{\prime} 10^{\prime \prime}$ & \\
\hline Run 36 & $153-157$ & $100 \%$ & & \\
\hline Run 37 & $157-164$ & $100 \%$ & & \\
\hline \multicolumn{5}{|l|}{$6-8-96$} \\
\hline Run 38 & $164-172$ & $100 \%$ & & \\
\hline Run 39 & $172-181$ & $100 \%$ & & \\
\hline Run 40 & $181-187^{\prime} 4^{\prime \prime}$ & $64^{\prime \prime}$ & $100 \%$ & \\
\hline Run 41 & $187^{\prime} 4 "-189^{\prime} 5^{\prime \prime}$ & $100 \%$ & & \\
\hline \multicolumn{5}{|c|}{ mud viscosity: $51 \mathrm{sec}$} \\
\hline Run 43 & $193-196$ & $100 \%$ & & \\
\hline Run 44 & $196-198^{\prime} 6^{\prime \prime}$ & $100 \%$ & & \\
\hline Run 45 & $198^{\prime} 6^{\prime \prime}-205$ & $5^{\prime}$ & $198^{\prime} 6^{\prime \prime}-205^{\prime}$ & \\
\hline Run 46 & $205-212$ & $6^{\prime} 6^{\prime \prime}$ & $205-211^{\prime} 6^{\prime \prime}$ & \\
\hline Run 47 & $212-217$ & 3'8" & $212-215^{\prime} 8^{\prime \prime}$ & \\
\hline Run 48 & $217-225$ & $6^{\prime \prime} 2^{\prime \prime}$ & $217-223^{\prime \prime} 2^{\prime \prime}$ & \\
\hline Run 49 & $225-232$ & $5^{\prime} 10^{\prime \prime}$ & $225-230^{\prime} 10^{\prime \prime}$ & \\
\hline Run 50 & $232-241$ & $6^{\prime} 10^{\prime \prime}$ & $232-238^{\prime} 10^{\prime \prime}$ & \\
\hline Run 51 & $241-245$ & $2^{\prime} 8^{\prime \prime}$ & $241-243^{\prime} 8^{\prime \prime}$ & \\
\hline Run 52 & $245-252$ & $5^{\prime} 5^{\prime \prime}$ & $245-250^{\prime} 5^{\prime \prime}$ & \\
\hline Run 53 & $252-256^{\prime \prime \prime}$ & $4^{\prime} 2^{\prime \prime}$ & $100 \%$ & \\
\hline Run 54 & $256^{\prime} 2^{\prime \prime}-257^{\prime}$ & $100 \%$ & & \\
\hline Run 55 & $257-264$ & $100 \%$ & & \\
\hline Run 56 & $264-268^{\prime} 5^{\prime \prime}$ & $3^{\prime} 7^{\prime \prime}$ & $264-267^{\prime} 7^{\prime \prime}$ & \\
\hline Run 57 & $268^{\prime} 5^{\prime \prime}-273$ & $100 \%$ & & \\
\hline \multicolumn{5}{|l|}{$6-9-96$} \\
\hline \multicolumn{5}{|c|}{ mud viscosity: $54 \mathrm{sec}$} \\
\hline Run 58 & $273-277$ & $100 \%$ & & \\
\hline Run 59 & $277-284$ & 6'4" & $277-283^{\prime} 4^{\prime \prime}$ & \\
\hline Run 60 & $284-292$ & $6^{\prime} 10^{\prime \prime}$ & $284-290^{\prime} 10^{\prime \prime}$ & \\
\hline Run 61 & $292-300$ & $6^{\prime} 10^{\prime \prime}$ & 292-298'10" & \\
\hline Run 62 & $300-306$ & $5^{\prime \prime \prime}$ & $300-305^{\prime} 2^{\prime \prime}$ & \\
\hline Run 63 & $306-313$ & $4^{\prime} 7^{\prime \prime}$ & $306-310^{\prime} 7^{\prime \prime}$ & \\
\hline Run 64 & $313-320$ & 6'3" & 313-319'3" & \\
\hline Run 65 & $320-326$ & $5^{\prime} 7^{\prime \prime}$ & $320-325^{\prime} 7^{\prime \prime}$ & \\
\hline Run 66 & $326-333$ & 3'9" & 326-329'9" & lost circulation 500 gals \\
\hline Run 67 & $333-336$ & $2^{\prime \prime} 2^{\prime \prime}$ & $333-335^{\prime \prime} 2^{\prime \prime}$ & \\
\hline Run 68 & $336-343$ & $5^{\prime} 6^{\prime \prime}$ & $336-341^{\prime} 6^{\prime \prime}$ & \\
\hline Run 69 & $343-351$ & $5^{\prime \prime} 3^{\prime \prime}$ & 343-347'3" & \\
\hline Run 70 & $351-358$ & $5^{\prime} 10^{\prime \prime}$ & $351-356^{\prime} 10^{\prime \prime}$ & \\
\hline Run 71 & $358-365$ & 6'1" & 358-364'1" & \\
\hline Run 72 & $365-373$ & $6^{\prime} 2^{\prime \prime}$ & 365-371'2" & \\
\hline
\end{tabular}


6-10-96

Run $73 \quad 373-380$

Run 74 380-386

Run 75 386-393

Run 76 393-395

Run 77 395-402

mud viscosity: $47 \mathrm{sec}$

Run 78 . 402-404'8"

Run 79 404'8"-413'4"

Run $80 \quad 413^{\prime} 4^{\prime \prime}-419$

Run 81 419-421

Run 82 421-426

Run 83 426-433

Run $84 \quad 433-440$

Run $85 \quad 440-447$

Run $86 \quad 447-453$

6-11-96

Run $87 \quad 453-460$

Run $88 \quad 460-464$

Run 89 464-471

Run $90 \quad 471-475$

Run $91 \quad 475-477$

Run $92 \quad 477-480$

Run $93 \quad 480-485$

Run 94 485-492

mud viscosity: $78 \mathrm{sec}$
Recovered:

6'3" 373-379'3"

5'4" 380-385'4"

$71 \quad 100 \%$

1'4" 393-394'4"

$5^{\prime} 1^{\prime \prime} \quad 395-400^{\prime} 1^{\prime \prime}$

mud weight: $8.8 \mathrm{lbs} / \mathrm{gal}$

2'4" 402-404'4"

8'7". 404'8"-413'3"

$5^{\prime} 6^{\prime \prime} \quad 413^{\prime} 4^{\prime \prime}-418^{\prime} 10^{\prime \prime}$

$100 \%$

$3^{\prime} 10^{\prime \prime} \quad 421-424^{\prime} 10^{\prime \prime}$

6' $5^{\prime \prime} \quad 426-4322^{\prime \prime}$

$5^{\prime} \quad 433-438$

6'6" 440-446'6"

5'8" 447-452'8"

mud weight: $8.8 \mathrm{lbs} / \mathrm{gal}$

6-20-96

Run 95 492-493'6"

Run 96 493'6"-498'

Run 97 498-501

Run 98 501-508

Run 99 508-509'4"

Run 100 509'4"-513

Run 101 513-517

$\begin{array}{ll}6^{\prime} 6^{\prime \prime} & 453-459^{\prime} 6^{\prime \prime} \\ 2^{\prime} 8^{\prime \prime} & 460-462^{\prime} 8^{\prime \prime} \\ 4^{\prime} 7^{\prime \prime} & 464-468^{\prime} 7^{\prime \prime} \\ 3^{\prime} & 471-474 \\ 1^{\prime} 10^{\prime \prime} & 475-476^{\prime} 10^{\prime \prime} \\ 2^{\prime} & 477-479 \\ 3^{\prime} 1^{\prime \prime} & 480-483^{\prime} 1^{\prime \prime} \\ 6^{\prime \prime} 2^{\prime \prime} & 485-491^{\prime} 2^{\prime \prime}\end{array}$

\section{6-21-96}

Run 102 517-521

Run 102A 521-523

Run 103 523-532

mud viscosity: $55 \mathrm{sec}$

Run 104 532-535

Run 105 535-541

Run 106 541-545

$\begin{array}{ll}100 \% & \\ 4^{\prime} 1^{\prime \prime} & 493^{\prime} 6^{\prime \prime}-497^{\prime} 11^{\prime \prime} \\ 1^{\prime} 8^{\prime \prime} & 498-499^{\prime \prime} 8^{\prime \prime} \\ 100 \% & \\ 100 \% & \\ 3^{\prime} & 509^{\prime} 4^{\prime \prime}-512^{\prime} 4^{\prime \prime} \\ 2^{\prime} 9^{\prime \prime} & 513-515^{\prime} 9^{\prime \prime}\end{array}$

1'3" 517-518'3"

$9^{\prime \prime} \quad 521-521^{\prime \prime} 9^{\prime \prime}$

no recovery

mud weight: $8.7 \mathrm{lbs} / \mathrm{gal}$

$\begin{array}{ll}2^{\prime} 7^{\prime \prime} & 532-534^{\prime} 7^{\prime \prime} \\ 2^{\prime} 7^{\prime \prime} & 535-537^{\prime \prime} 7^{\prime \prime} \\ 3^{\prime} 4^{\prime \prime} & 541-544^{\prime} 4^{\prime \prime}\end{array}$


6-21-96

Run 107 545-552

Run 108 552-557

Run 109 557-562

\section{6-23-96}

Run $110562-567$

Run 111 567-568

Run 112 568-568'4"

Run 113 568'4"-572

Run 114 572-576

Run 115 576-580

Run 116 580-586

Run 117 586-592

Run 118 592-595

Run 119 595-595'6"

Run 120 595'6"-597

6-25-96

Run $121597-600$

Run 122 600-603'6"

Run 123 603'6"-605

Run 124 605-607

Run 125 607-611

Run 126 611-615

Run 127 615-616

Run 128 616-618

Run 129 618-620

6-28-96

Run 130 620-625

Run 131 625-629

Run 132 629-632

Run 133 632-635

Run 134 635-640

Run 135 640-645

Run 136 645-648

Run 137 648-651

Run 138 651-655

Run 139 655-657

Run $140657-660$

Run 141 660-665

Run 142 665-671

Run 143 671-673

$$
\begin{array}{ll}
\multicolumn{2}{c}{\text { Recovered: }} \\
6^{\prime} & 545-551 \\
4^{\prime} 10^{\prime \prime} & 552-556^{\prime} 10^{\prime \prime} \\
3^{\prime} & 557-560
\end{array}
$$

$\begin{array}{ll}2^{\prime} 10^{\prime \prime} & 562-564^{\prime} 10^{\prime \prime} \\ 9^{\prime \prime} & 567-567^{\prime} 9^{\prime \prime} \\ \text { no recovery } \\ \text { no recovery } \\ 3^{\prime \prime} & 572-575 \\ 3^{\prime} 3^{\prime \prime} & 576-579^{\prime} 3^{\prime \prime} \\ 4^{\prime} 5^{\prime \prime} & 580-584^{\prime} 5^{\prime \prime} \\ 3^{\prime} 7^{\prime \prime} & 586-589^{\prime} 7^{\prime \prime} \\ 1^{\prime} 1^{\prime \prime} & 592-593^{\prime} 1^{\prime \prime} \\ \text { no recovery } \\ 1^{\prime \prime} & 596-596^{\prime} 5^{\prime \prime}\end{array}$

$1^{\prime} 6^{\prime \prime} \quad 597-598^{\prime} 6^{\prime \prime}$

2'1" 600-602'1"

5" 604'7"-605

$100 \%$

$2^{\prime} 9^{\prime \prime} \quad 607-609^{\prime} 9^{\prime \prime}$

10" $614^{\prime \prime} 2^{\prime \prime}-615$

$100 \%$

$5^{\prime \prime} \quad 616-616^{\prime} 5^{\prime \prime}$

no recovery

$\begin{array}{ll}4^{\prime} 11^{\prime \prime} & 620-624^{\prime} 11^{\prime \prime} \\ 3^{\prime} 2^{\prime \prime} & 625-628^{\prime} 2^{\prime \prime} \\ 2^{\prime} 8^{\prime \prime} & 629-631^{\prime} 8^{\prime \prime} \\ 100 \% & \\ 100 \% & \\ 4^{\prime} 5^{\prime \prime} & 640^{\prime} 7^{\prime \prime}-645 \\ 100 \% & \\ 100 \% & \\ 100 \% & \\ 100 \% & \\ 28^{\prime \prime} & 657-659^{\prime} 8^{\prime \prime} \\ 100 \% & \\ 57^{\prime \prime} \text { lost } 666^{\prime}-666^{\prime} 5^{\prime \prime} \\ 100 \% & \end{array}$


6-29-96

Run 144 673-675

Run 145 675-676

Run 146 676-680

Run 147 680-683'3"

Run 147 683'3"-684

$\mathrm{TD}=684^{\prime}$
Recovered:

4'1" $\quad 100 \%$

11" 675-675'11"

3'10" 676-679'10"

$100 \%$

$100 \%$ 


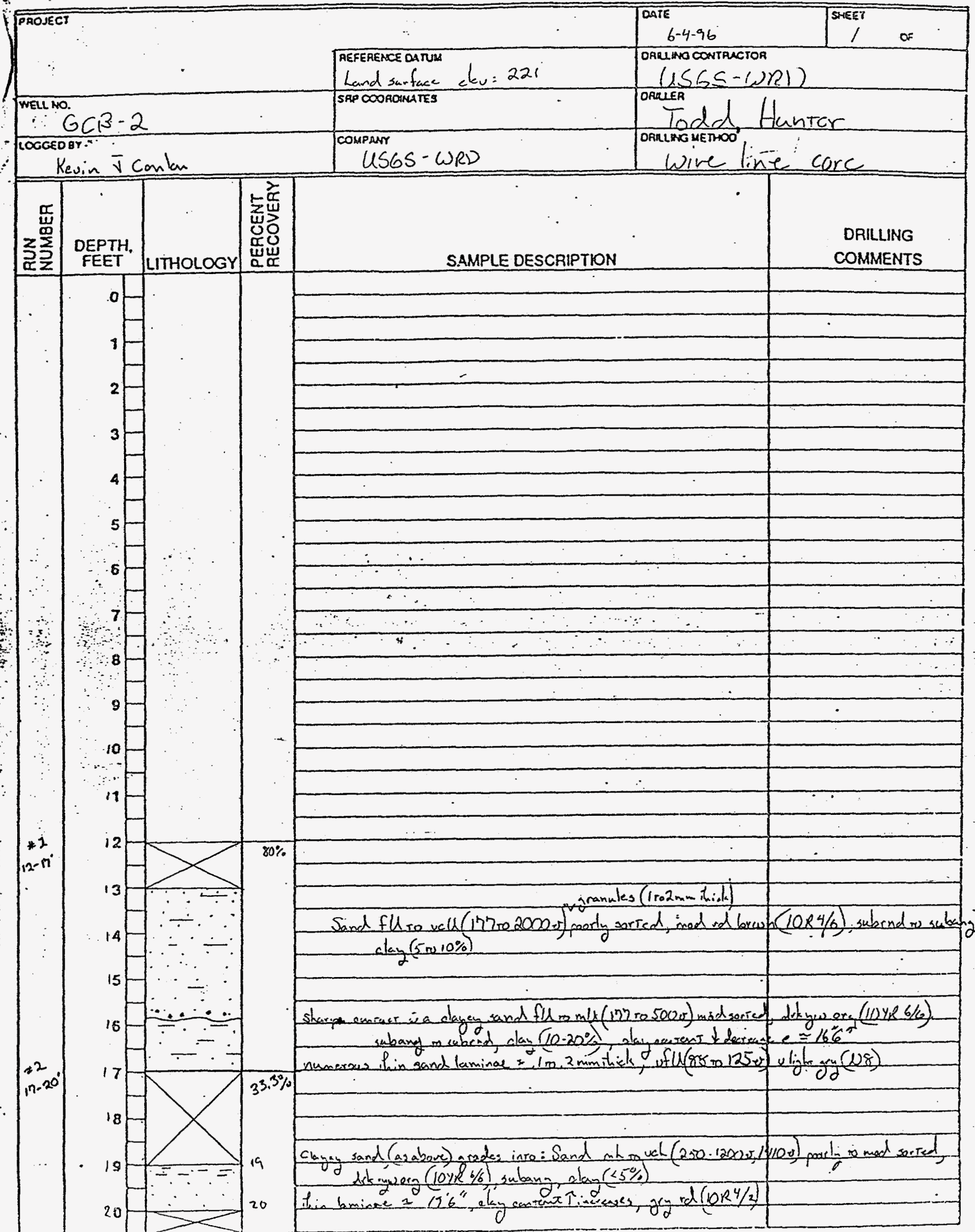




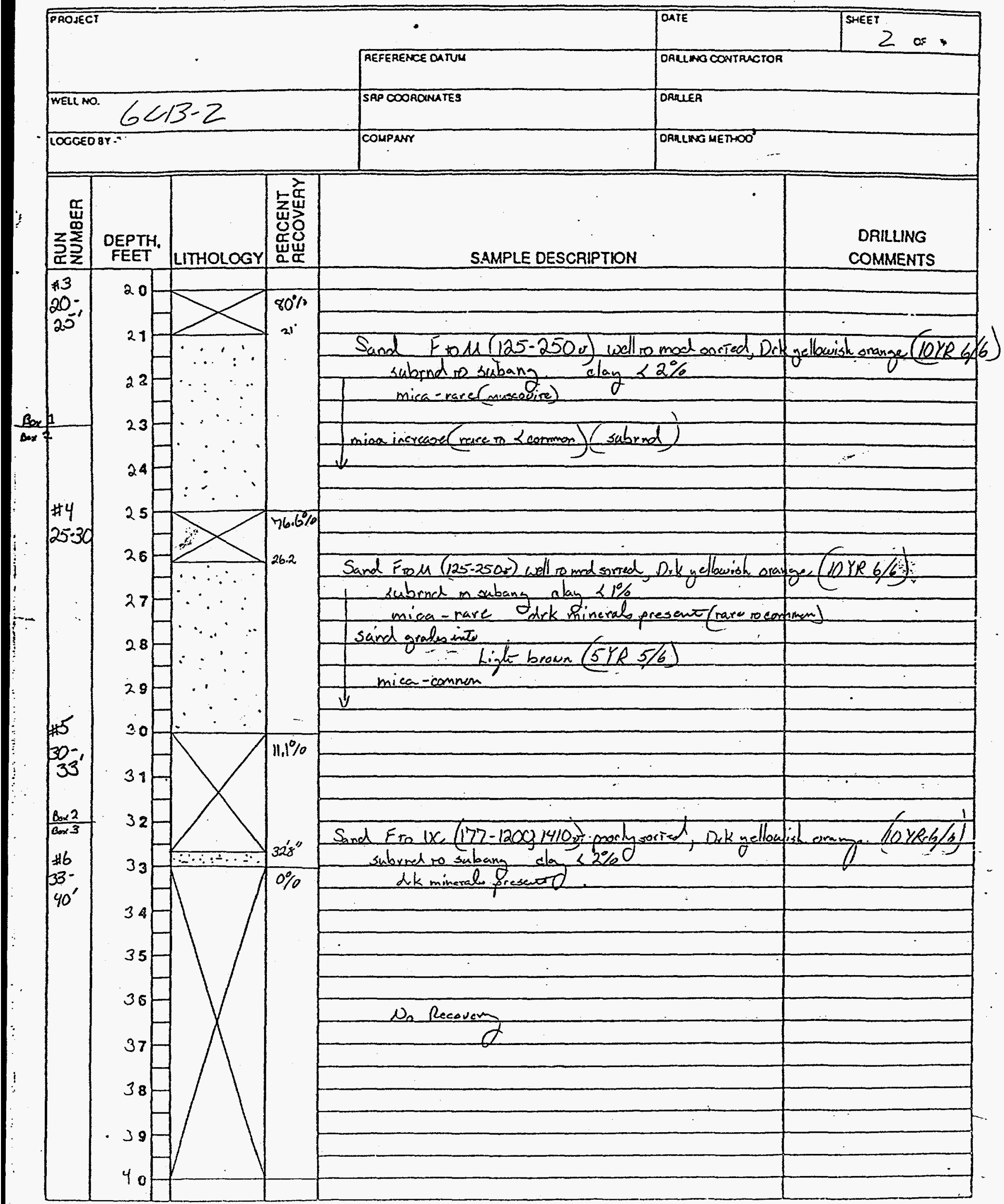




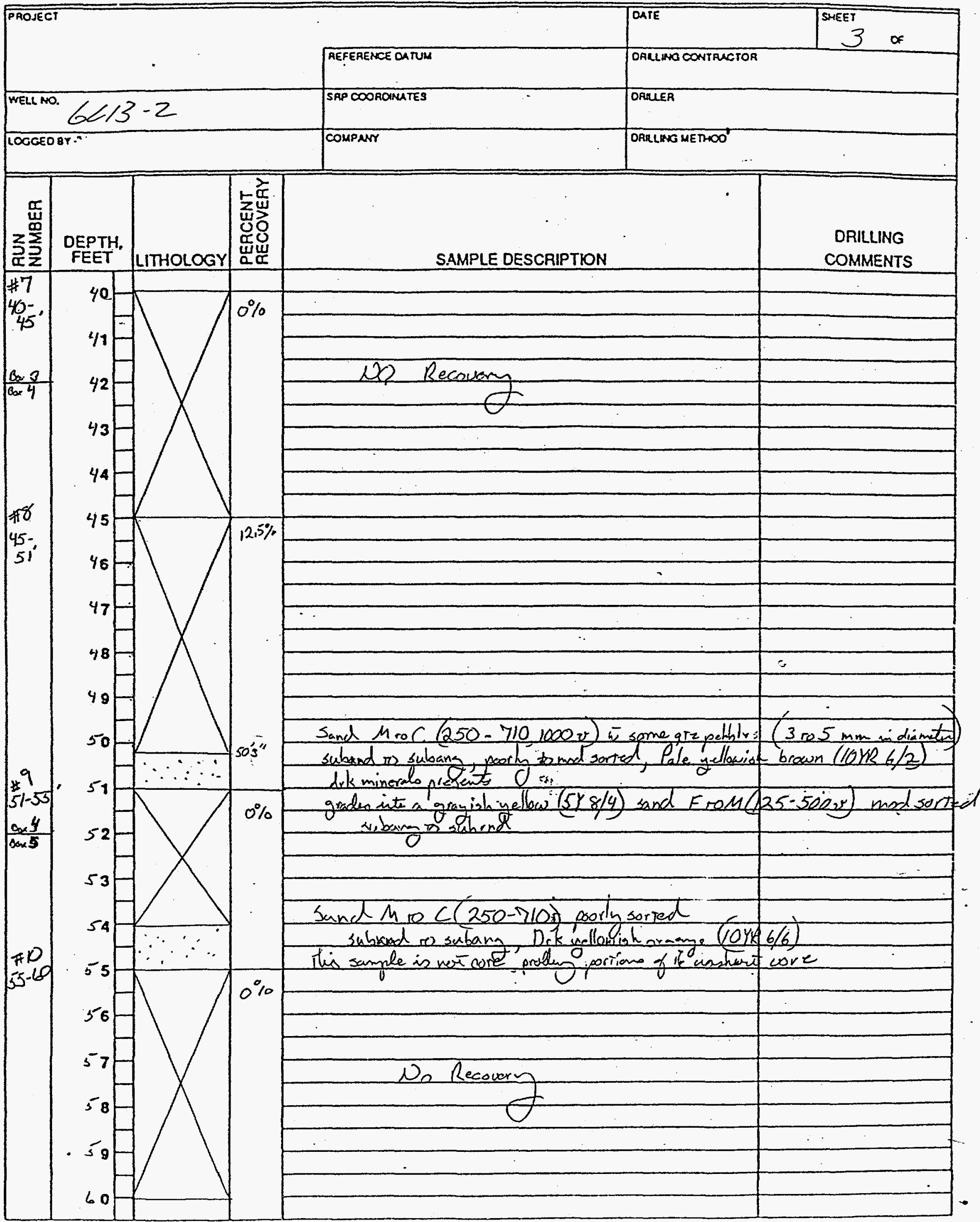




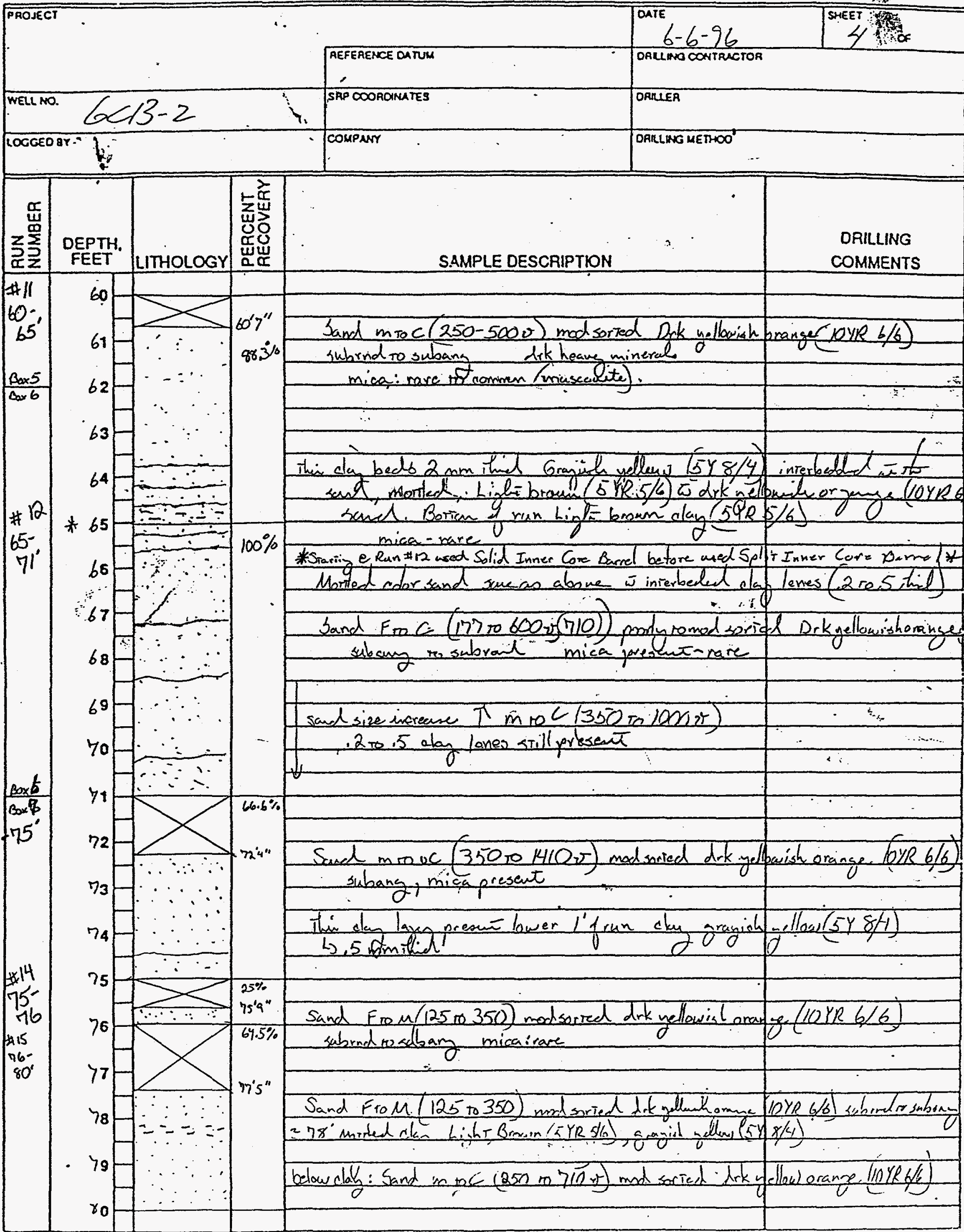


FIELD GEOLOGIC LOG

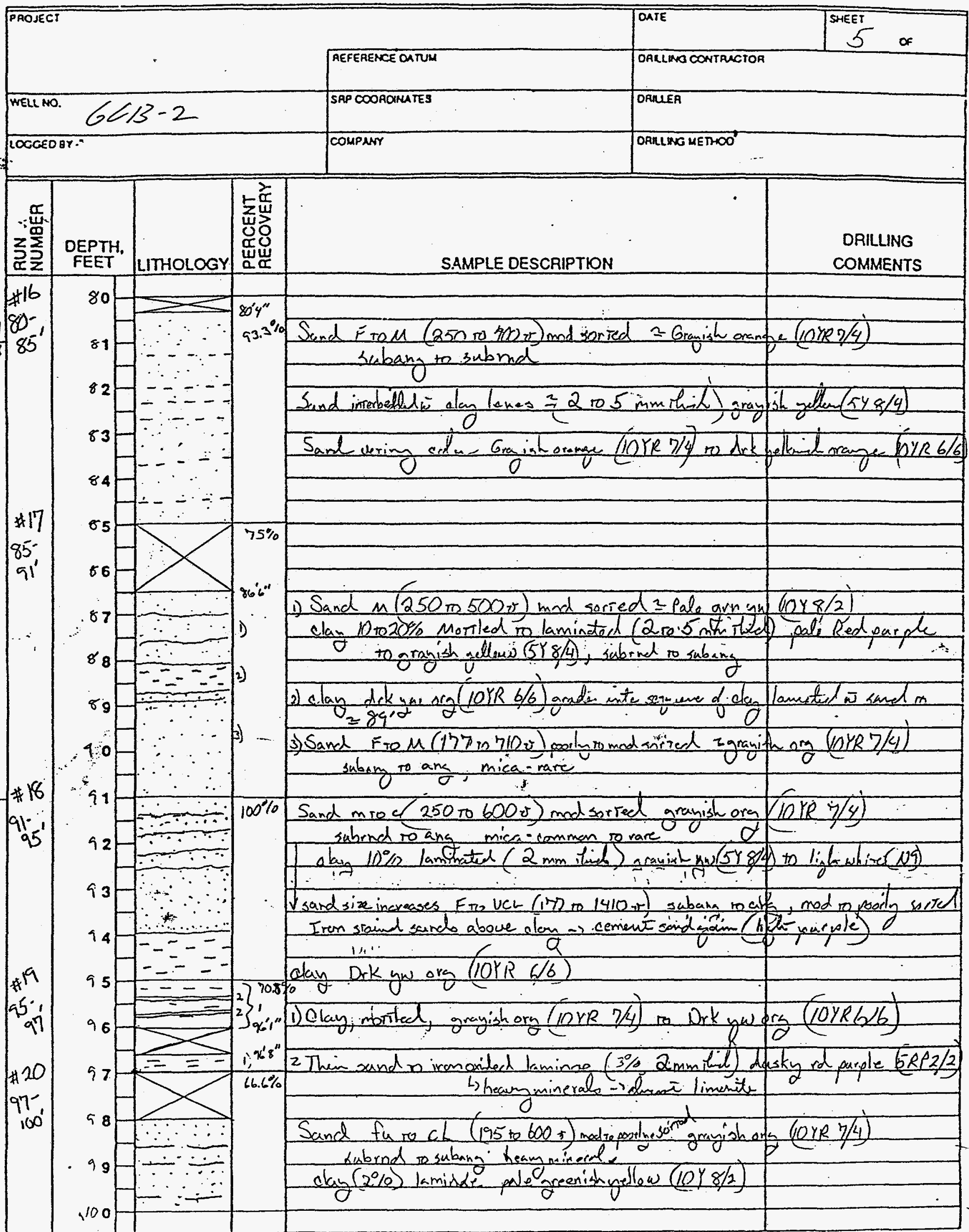


FIELD GEOLOGIC LOG

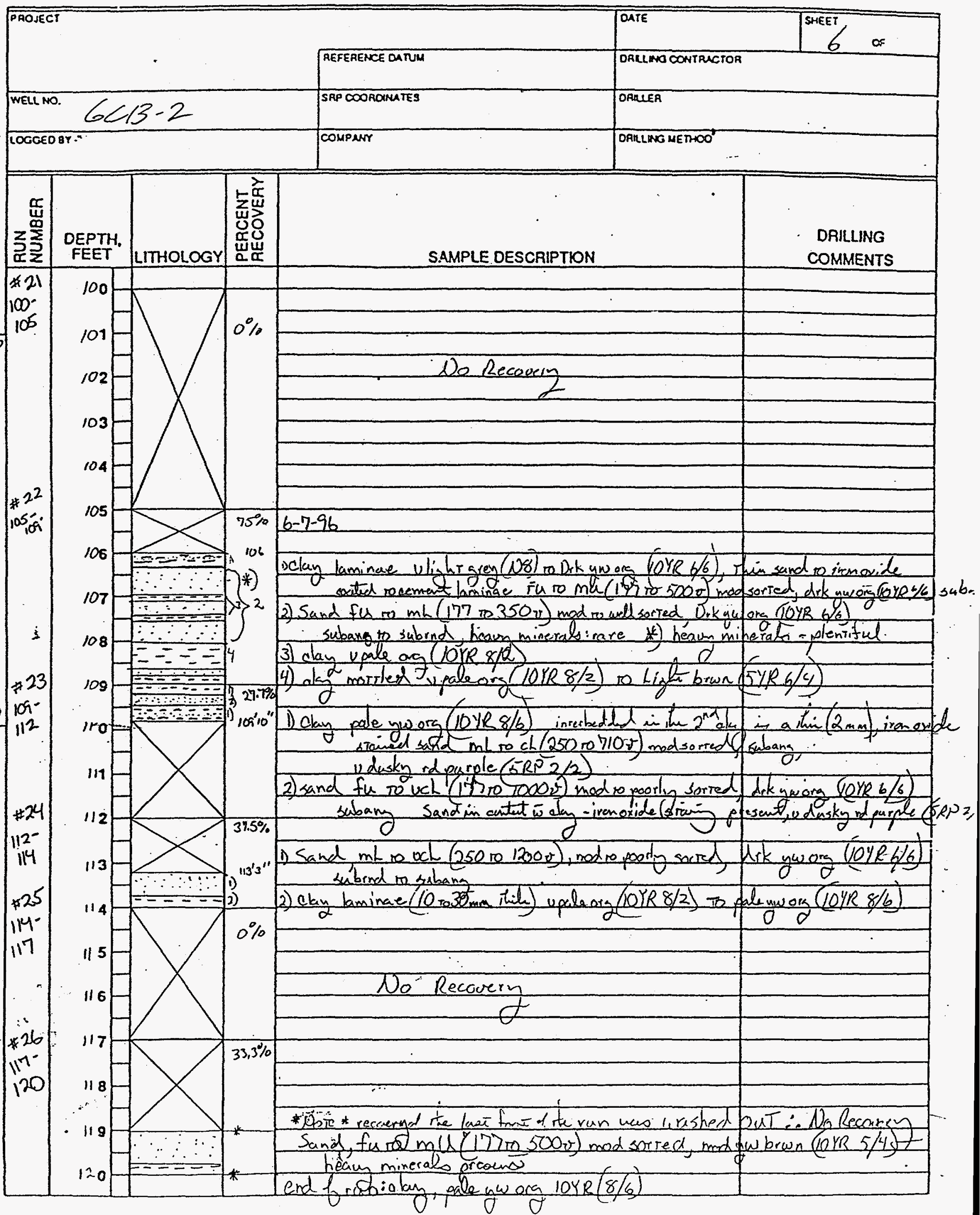


FIELD GEOLOGIC LOG






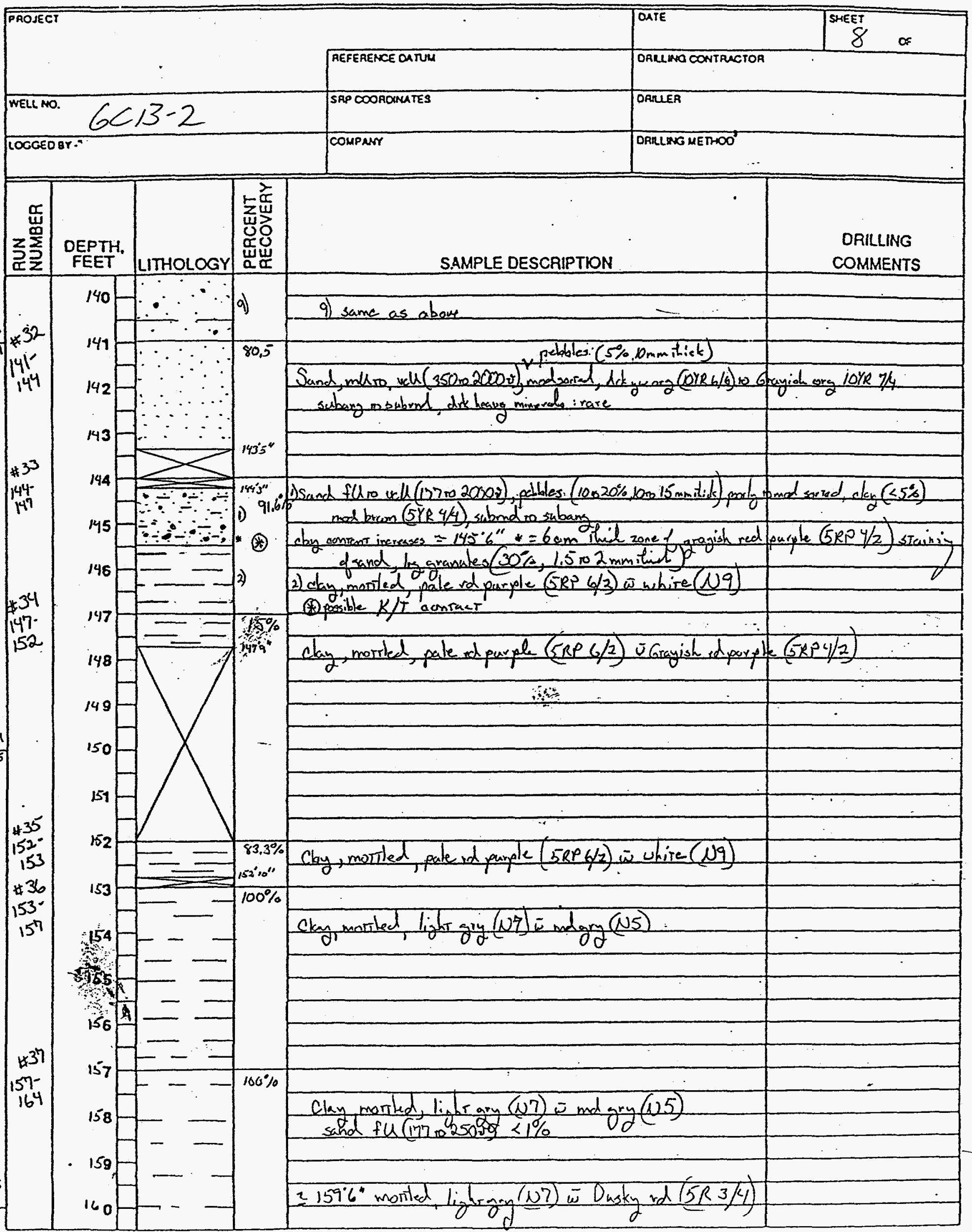


FIELD GEOLOGIC LOG

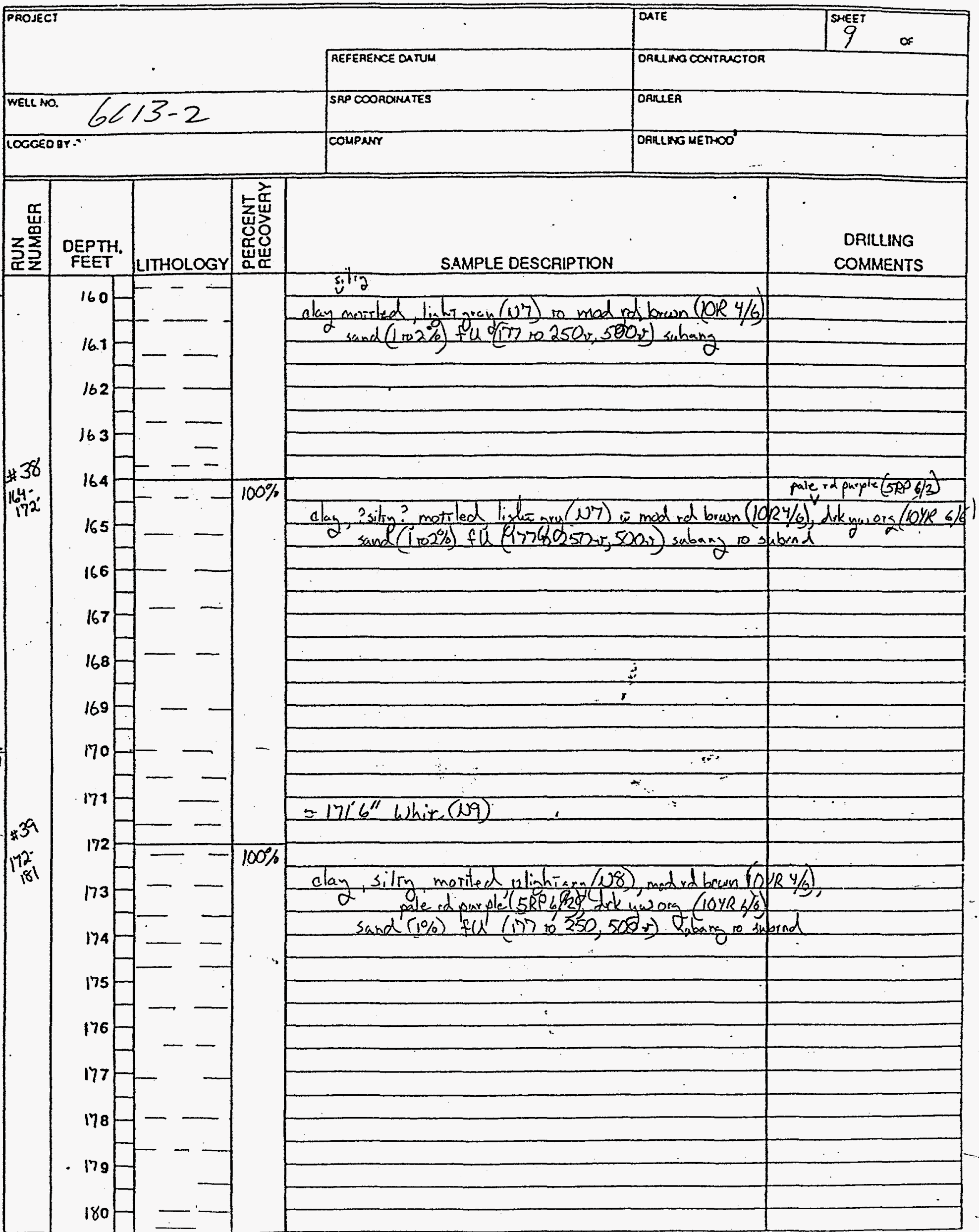


FIELD GEOLOGIC LOG

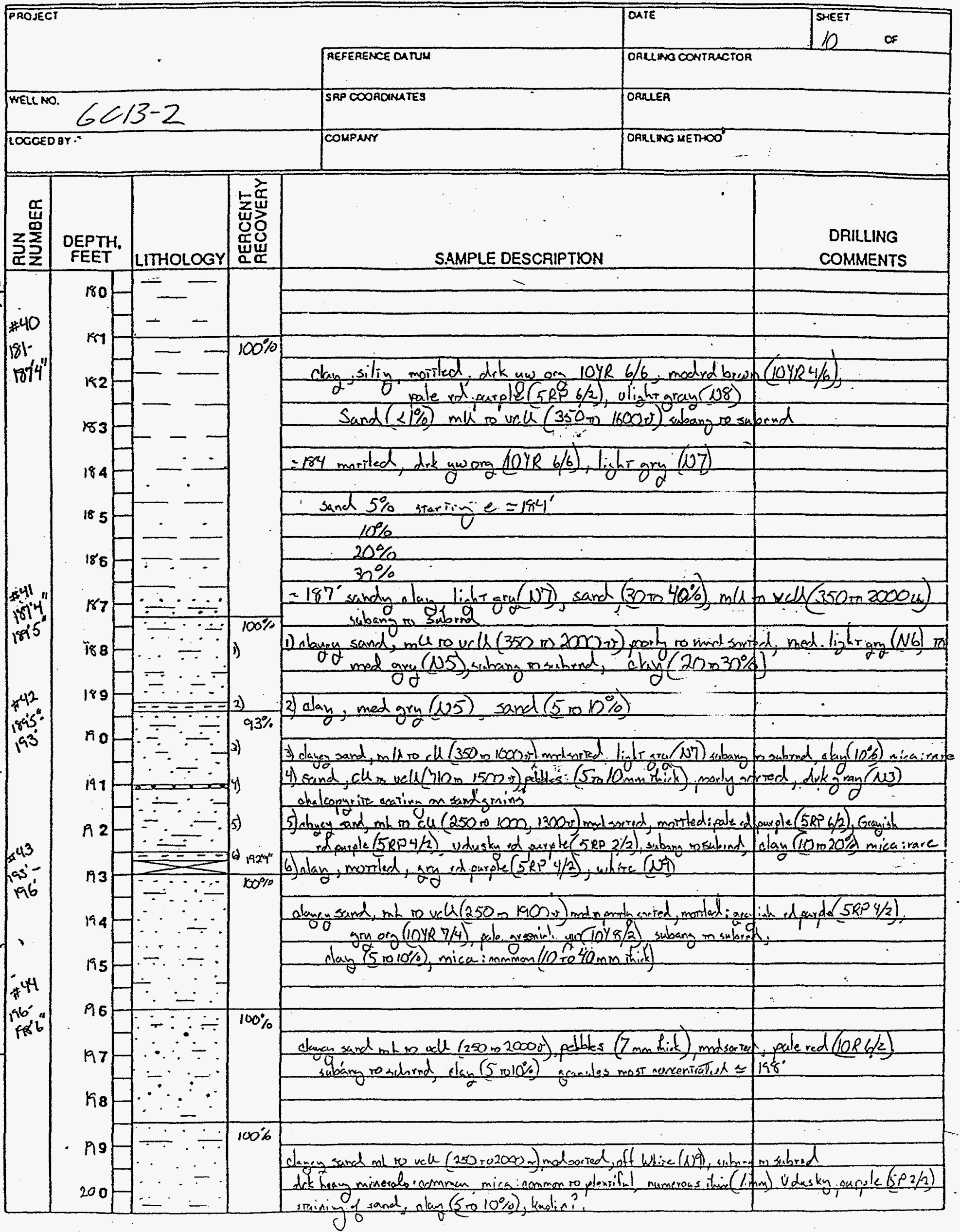




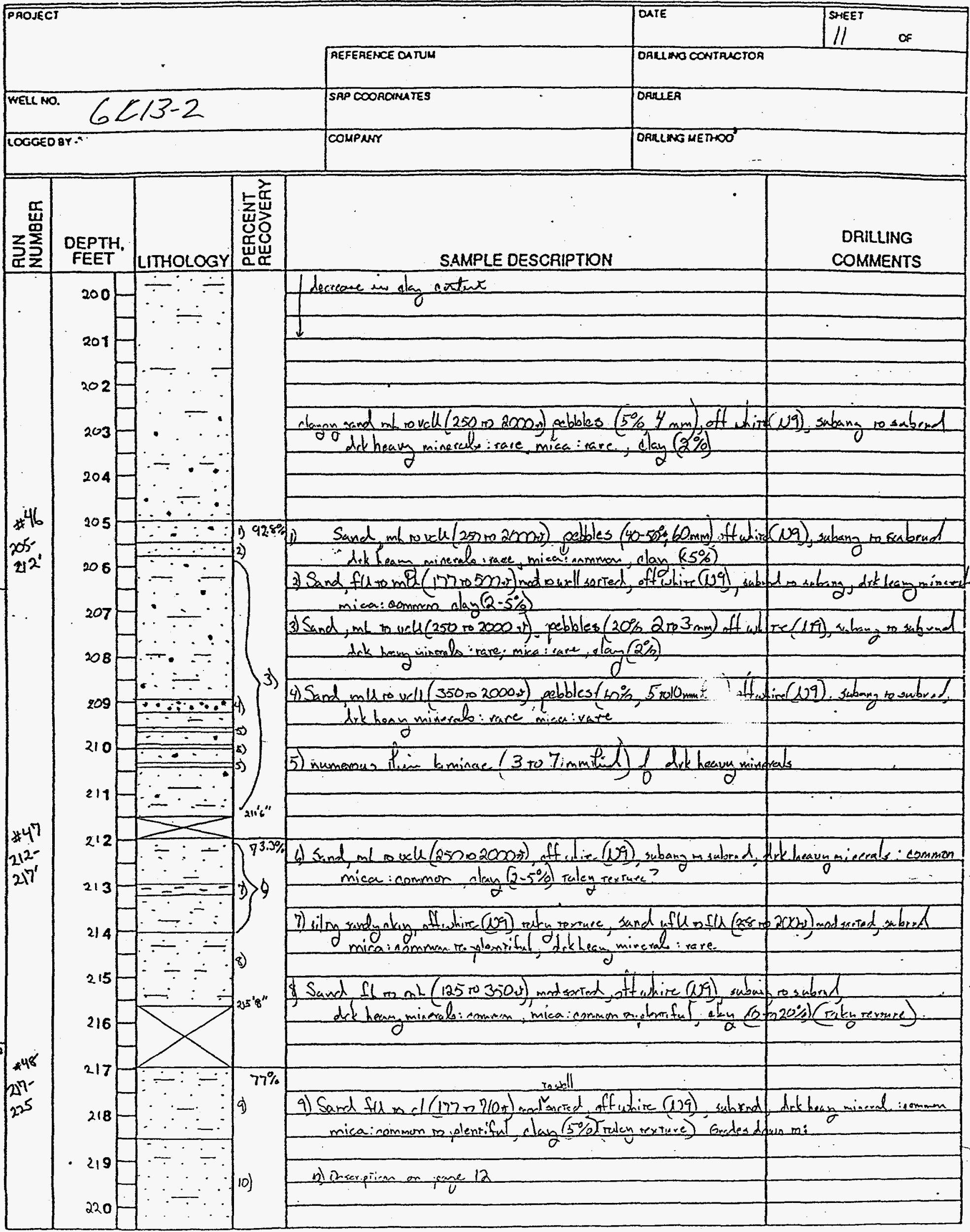


FIELD GEOLOGIC LOG

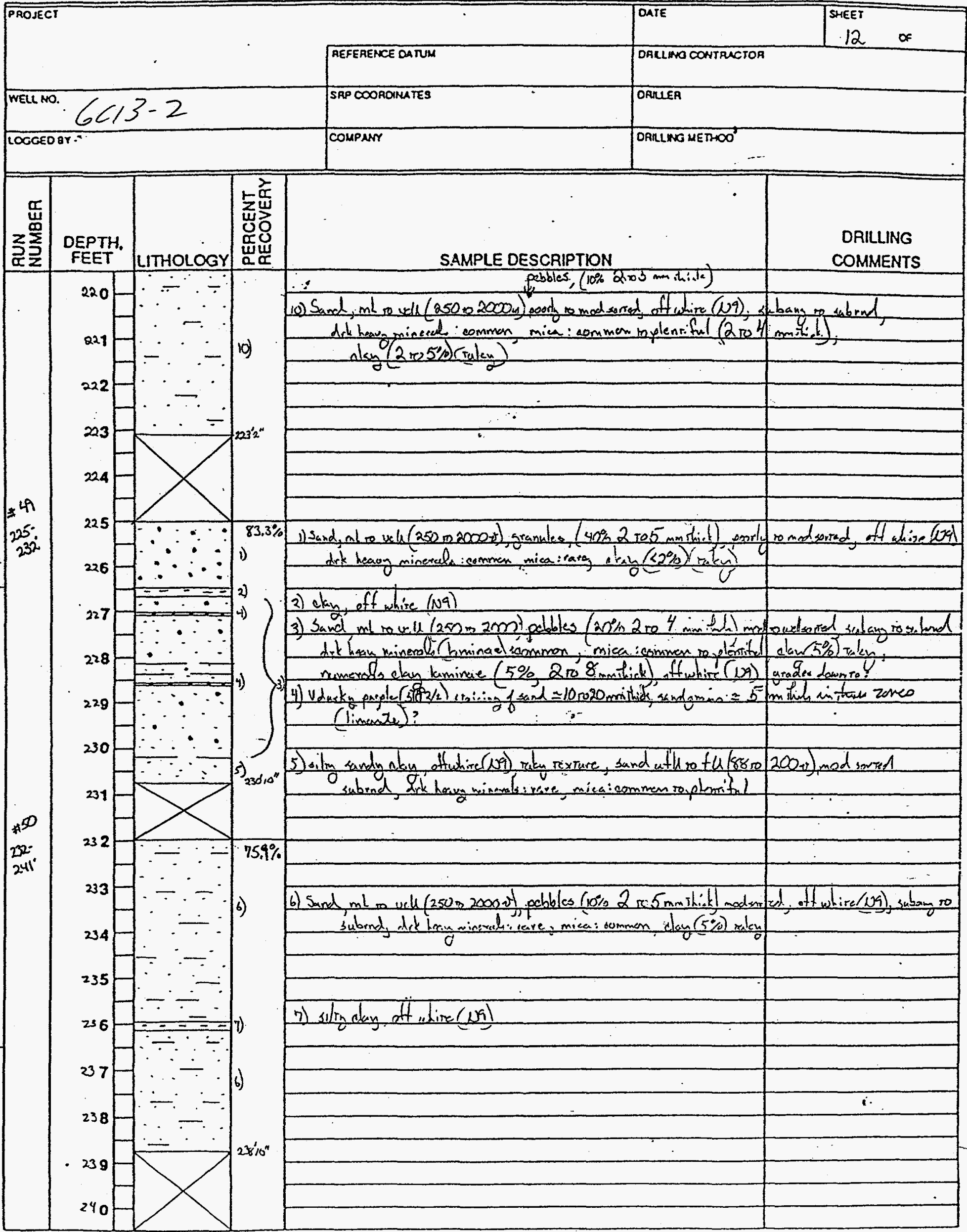




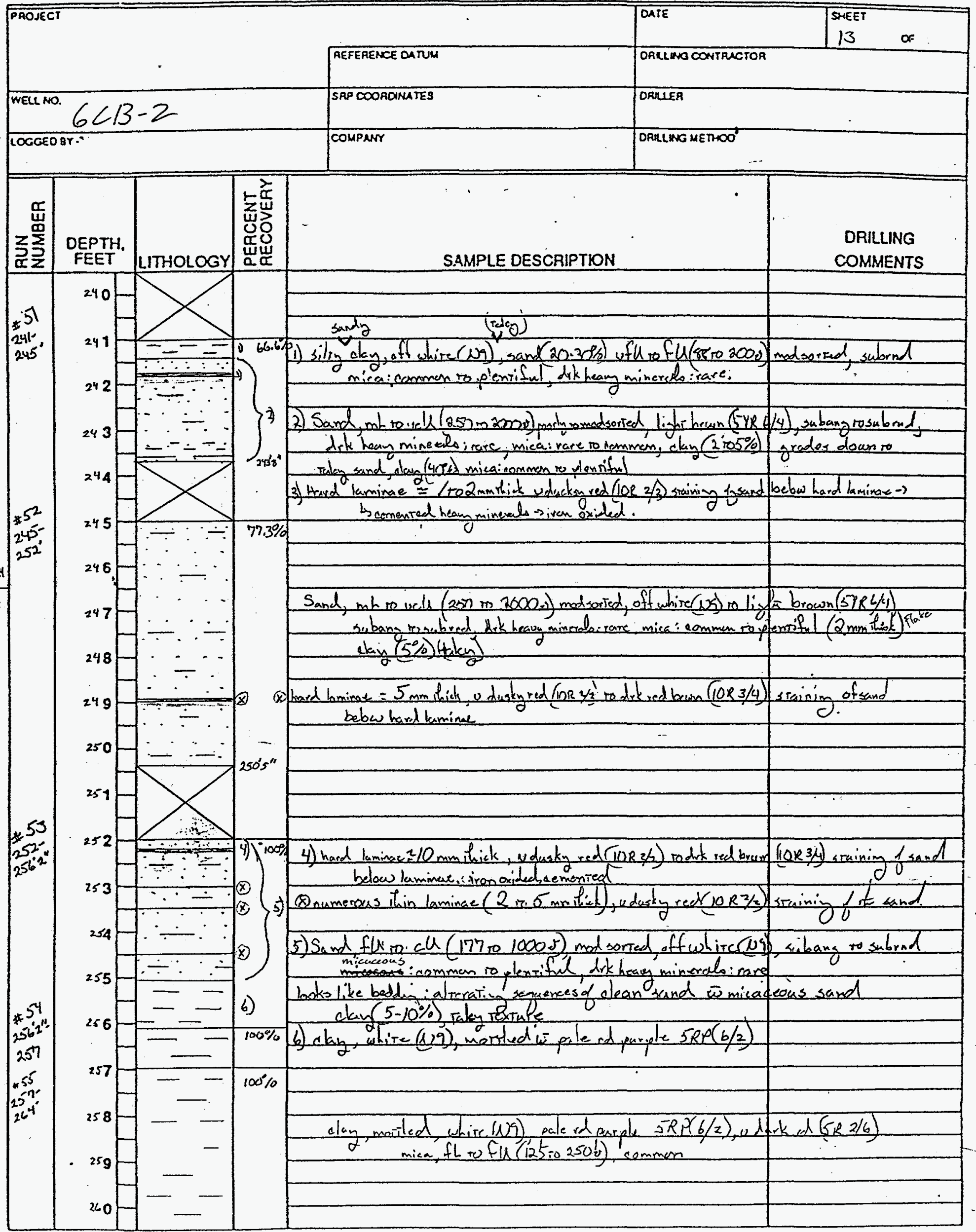


FIELD GEOLOGIC LOG

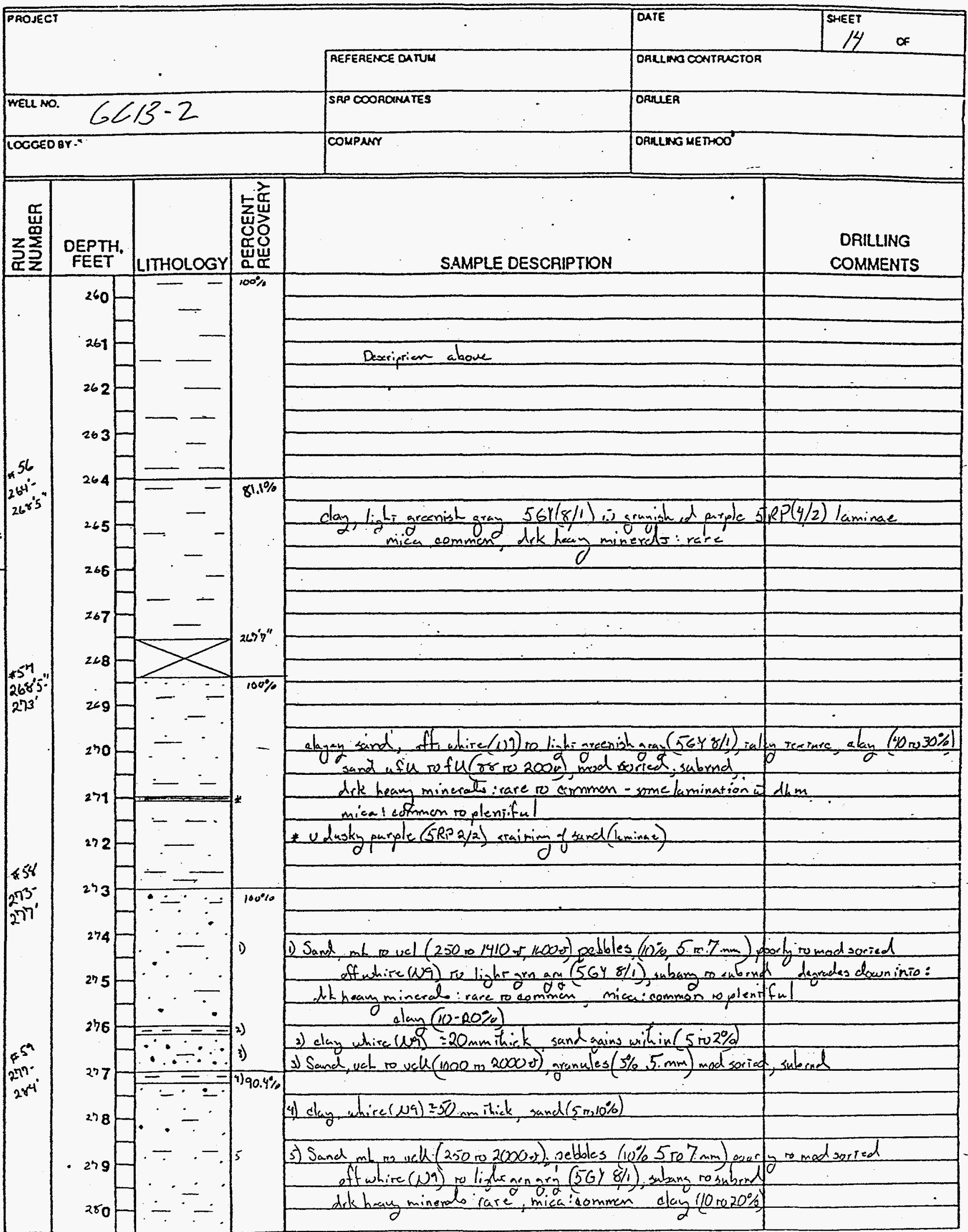









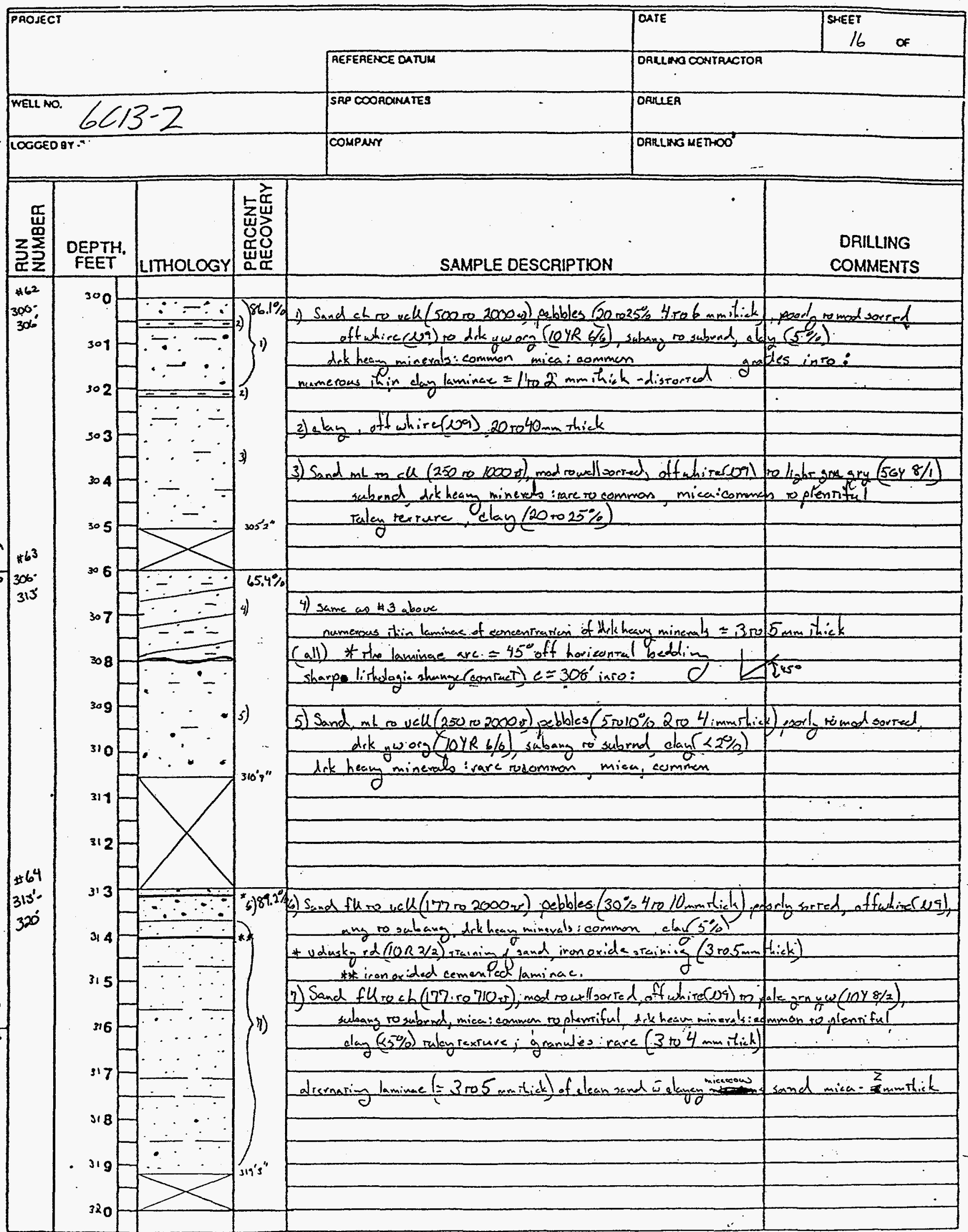


FIELD GEOLOGIC LOG

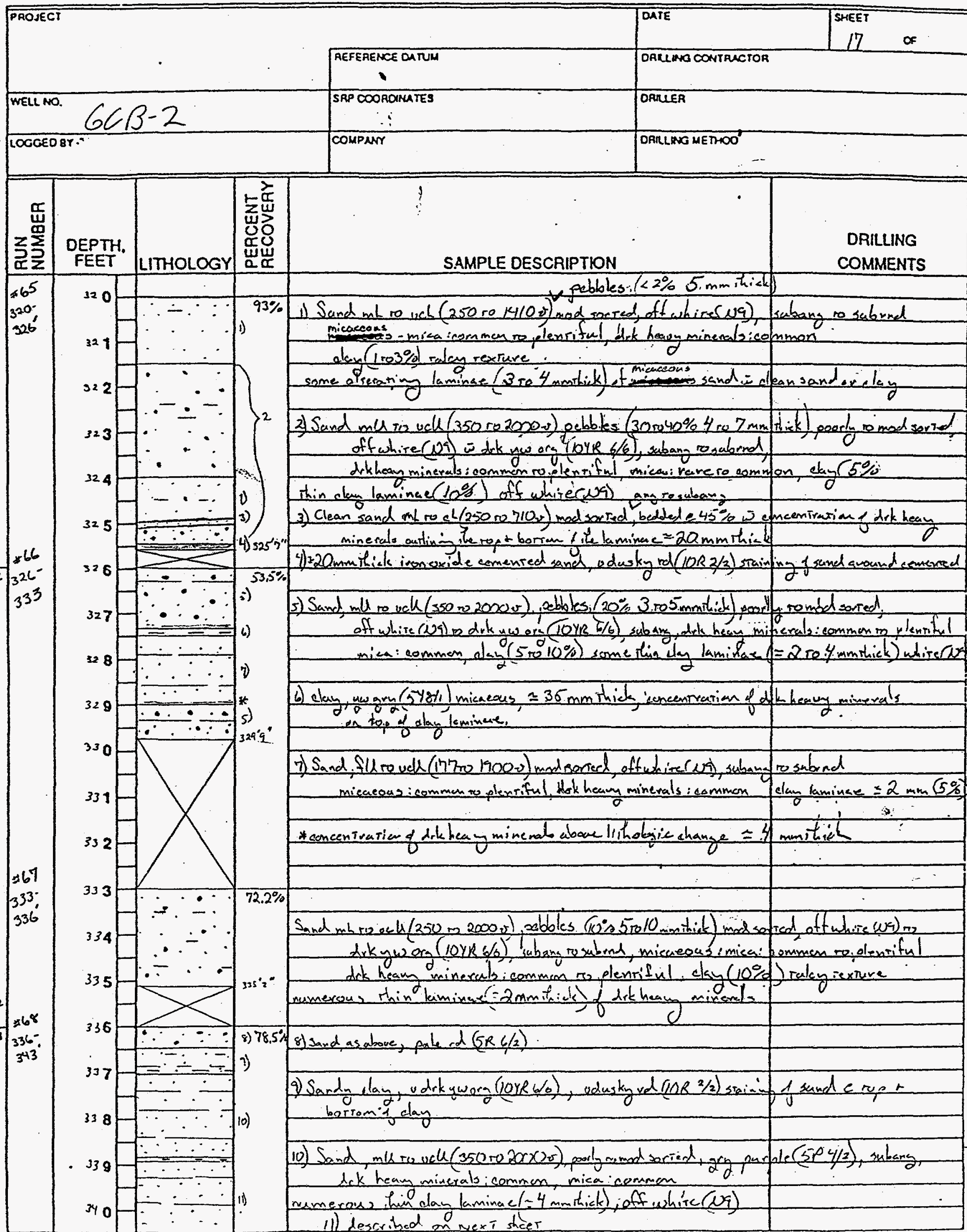




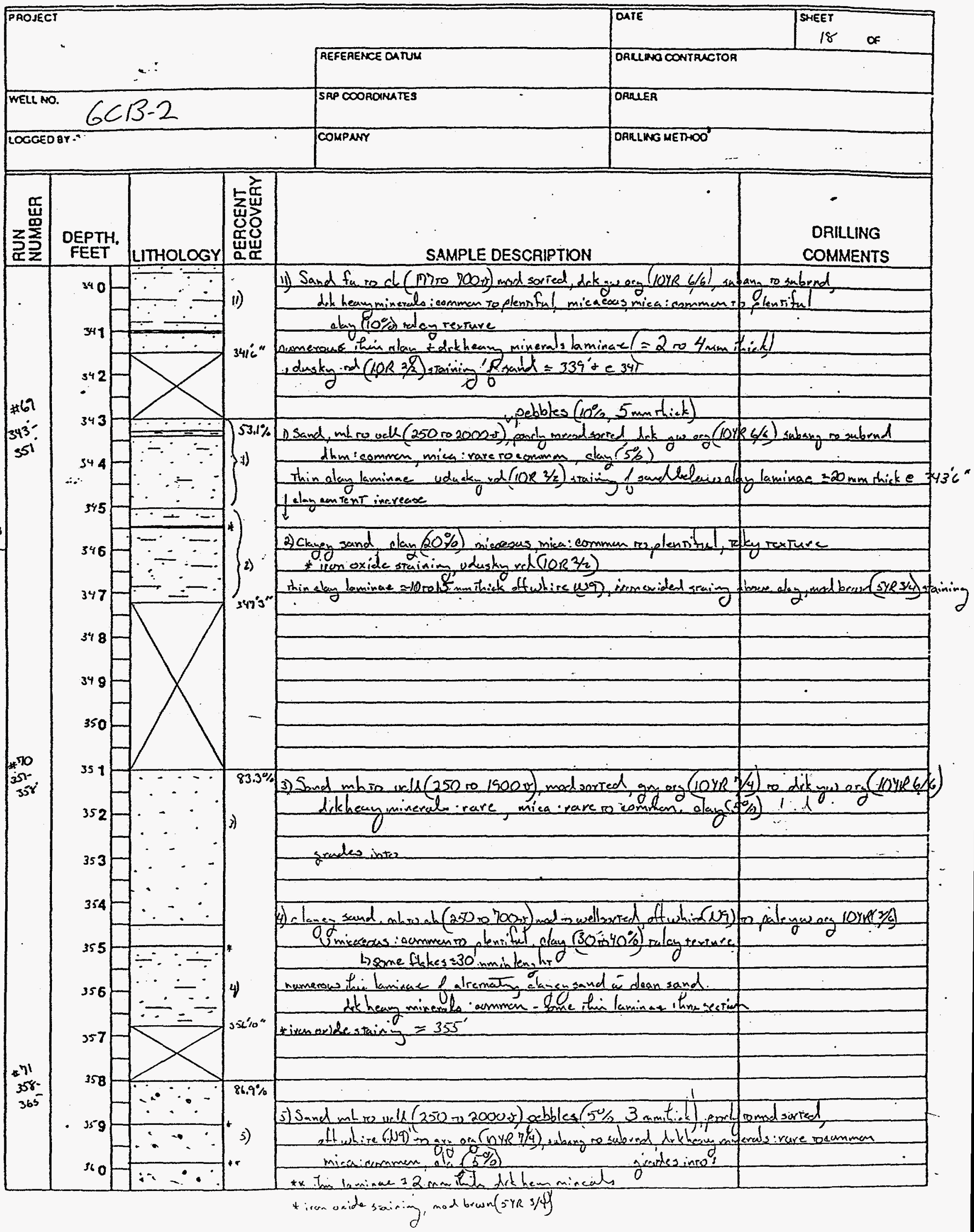




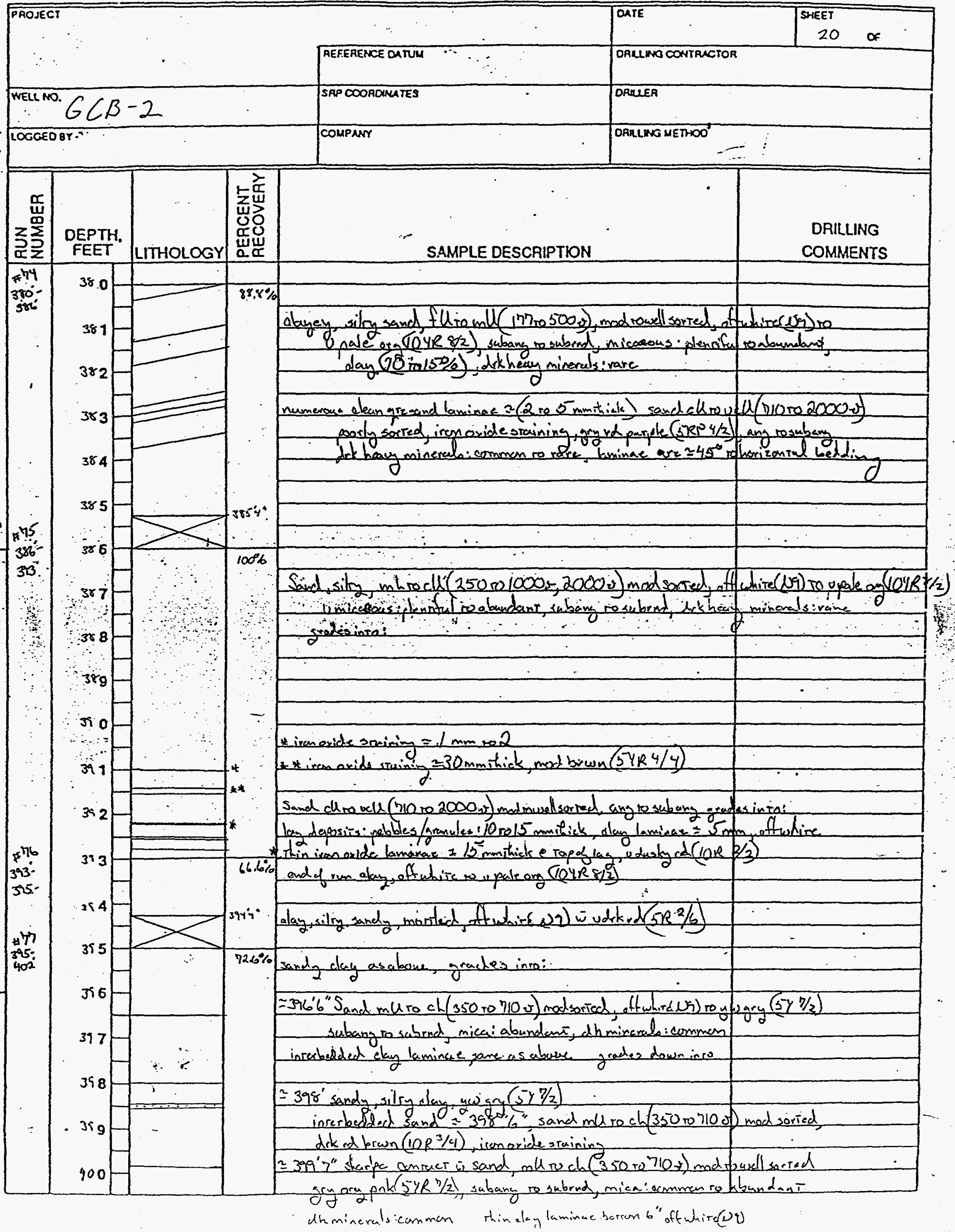




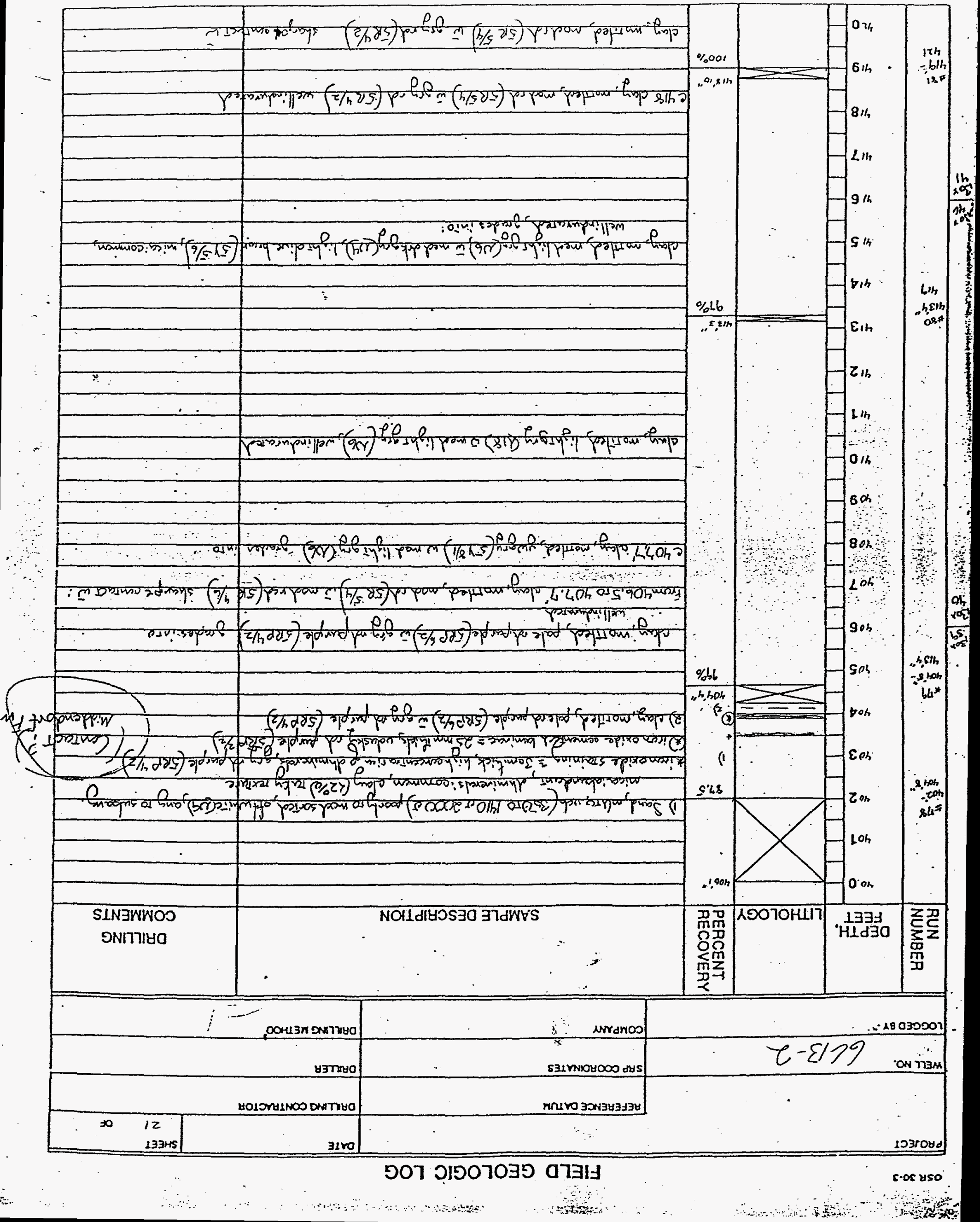




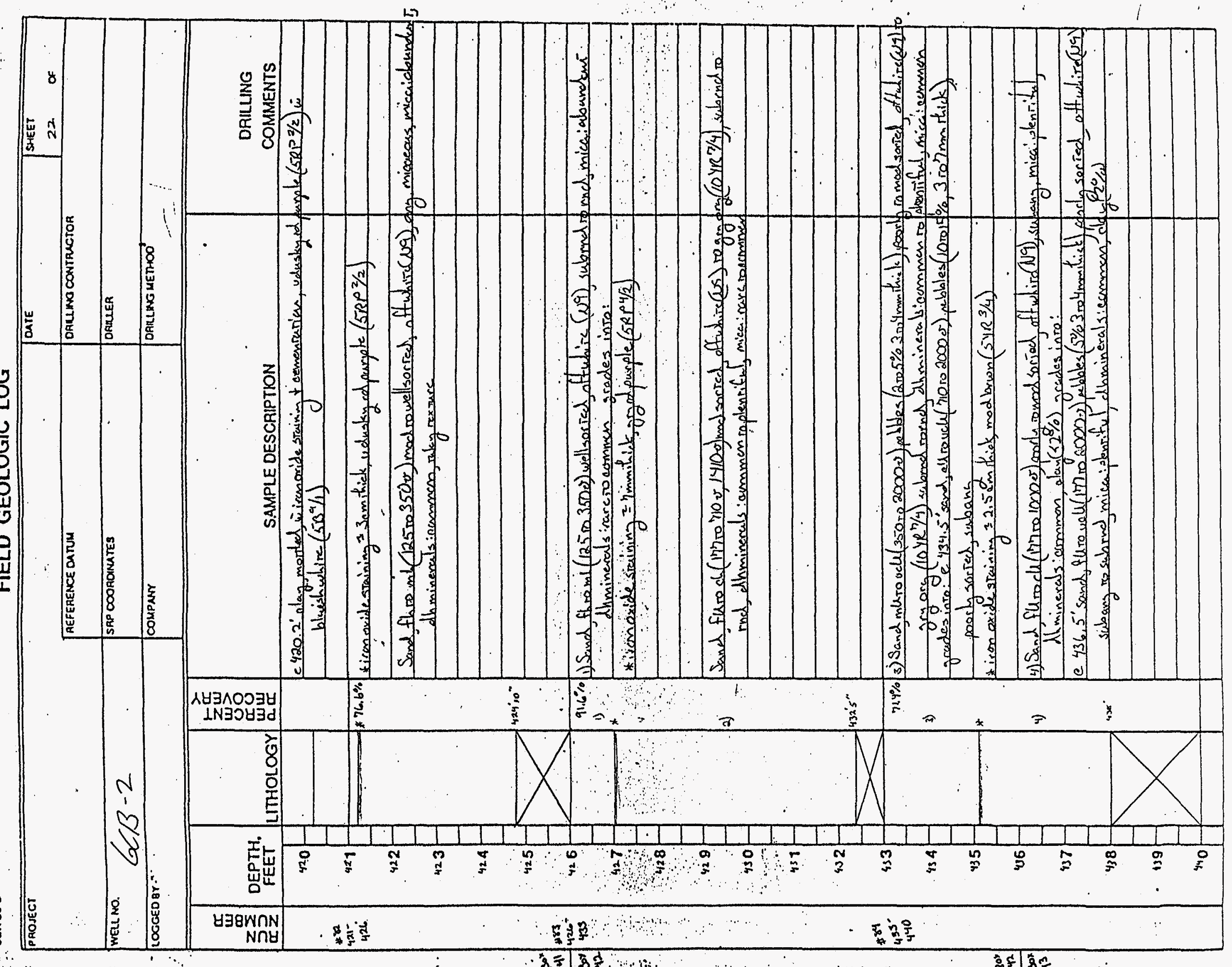

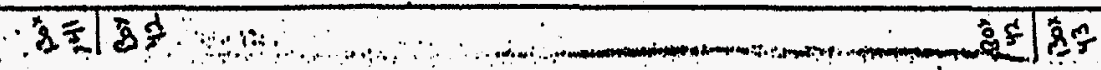




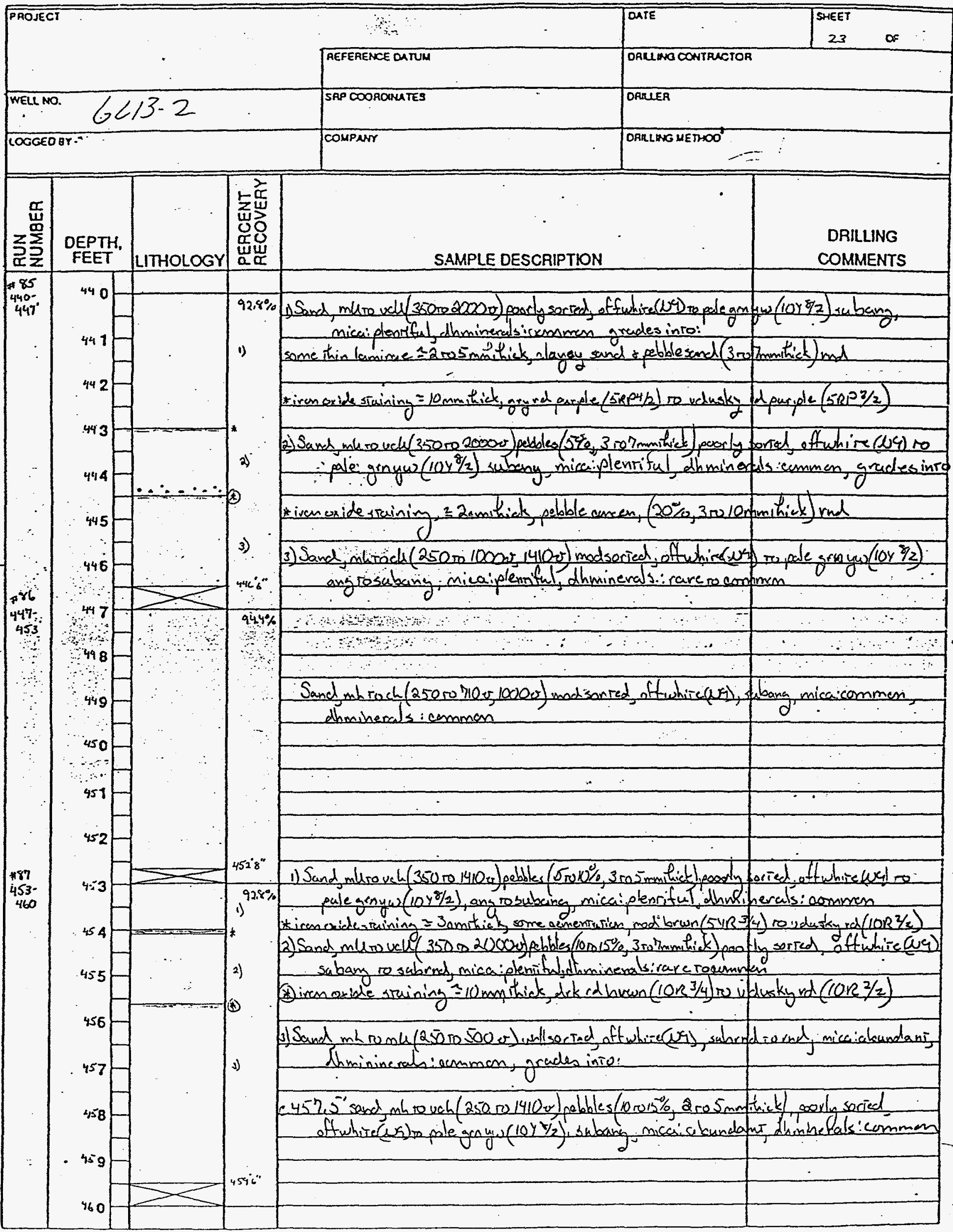


xy

OSR 30.3

FIELD GEOLOGIC LOG

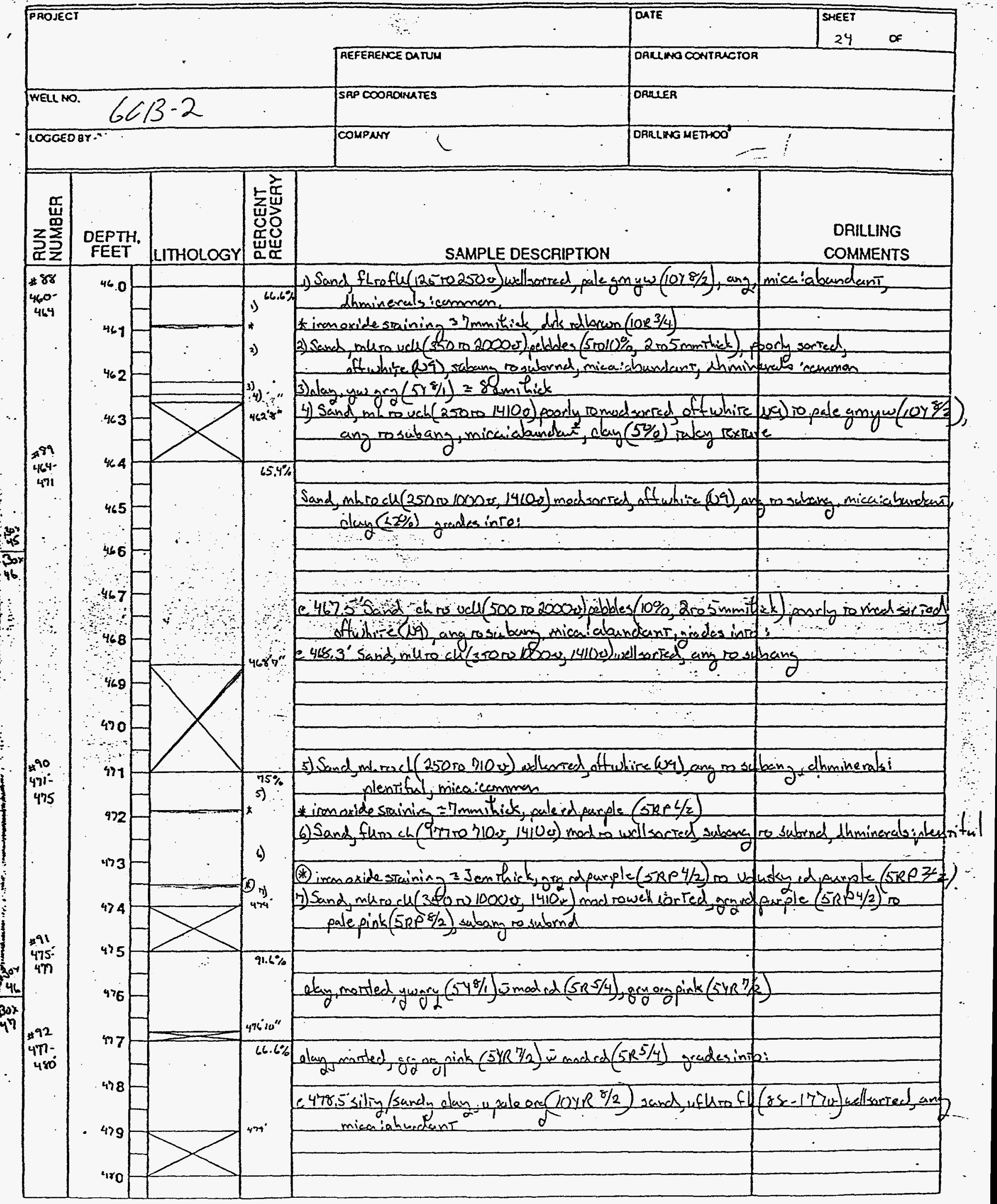




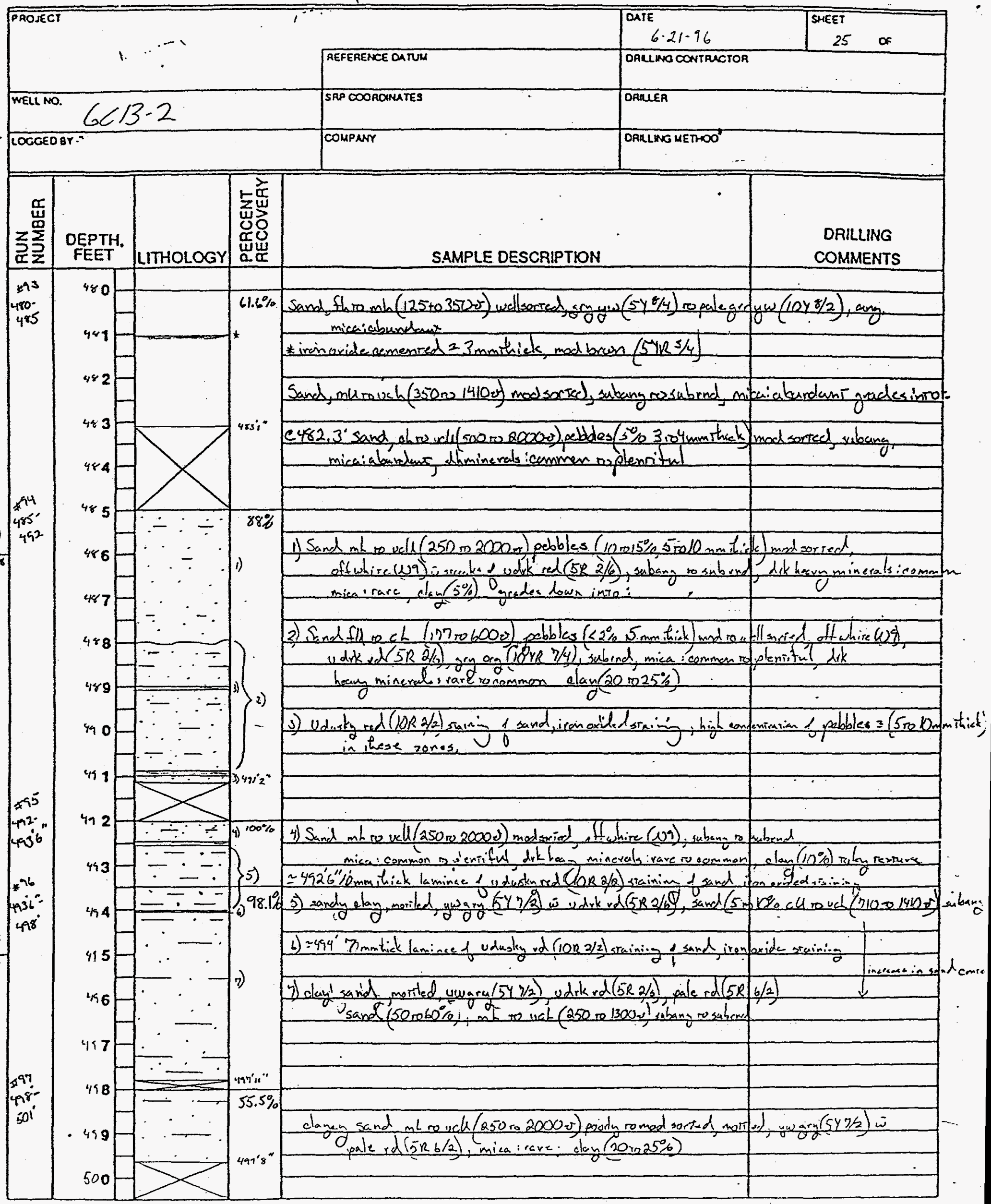




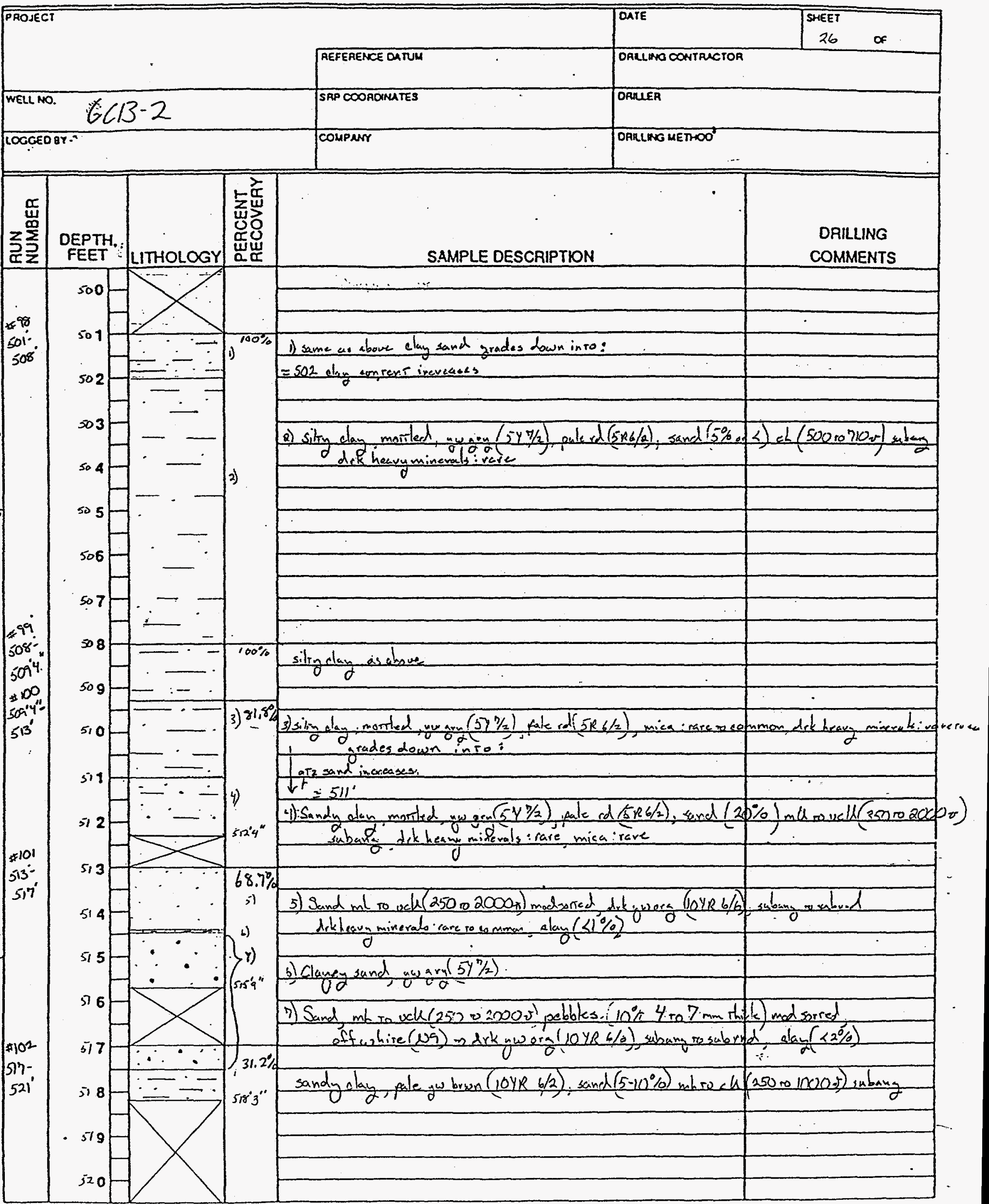


FIELD GEOLOGIC LOG

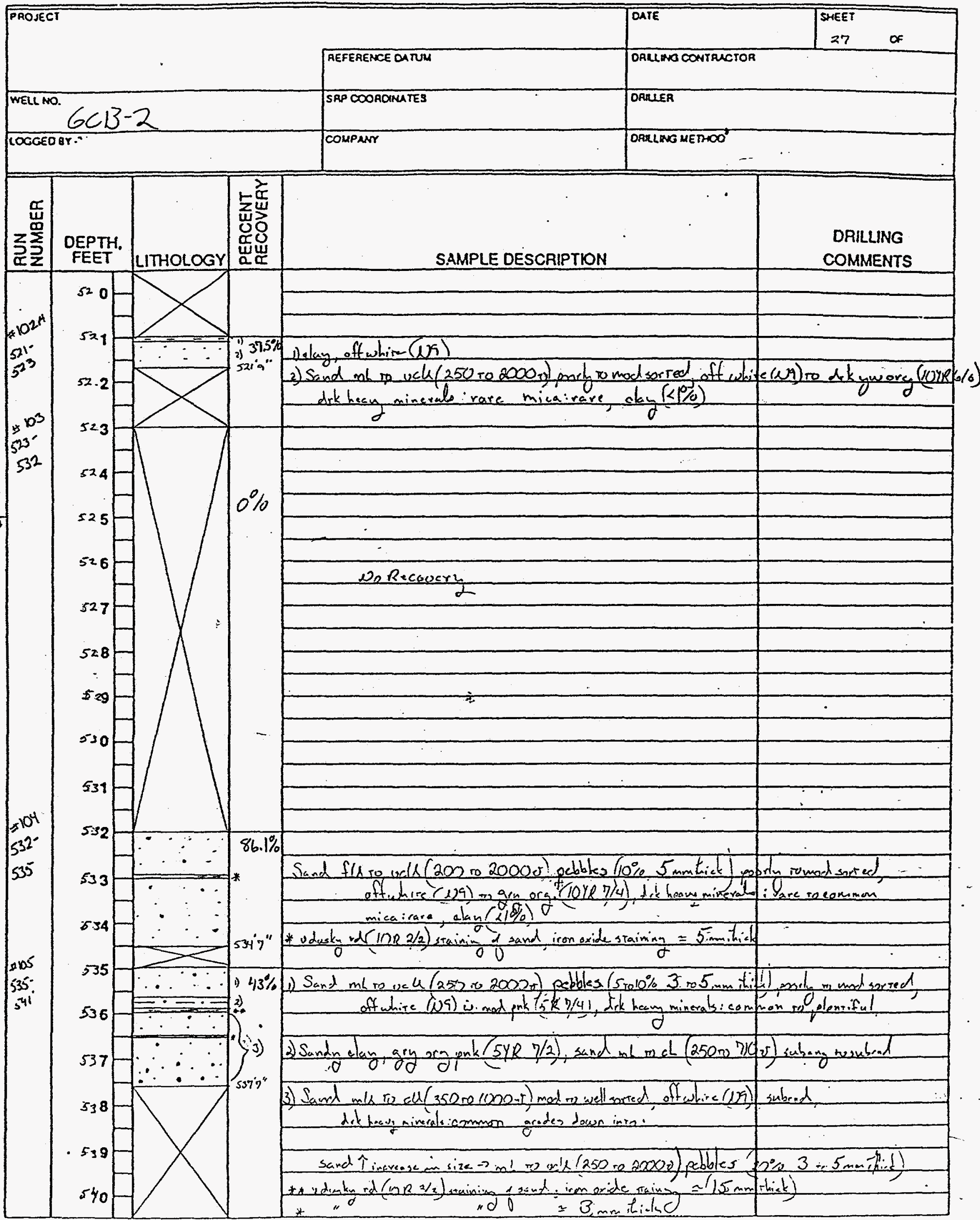




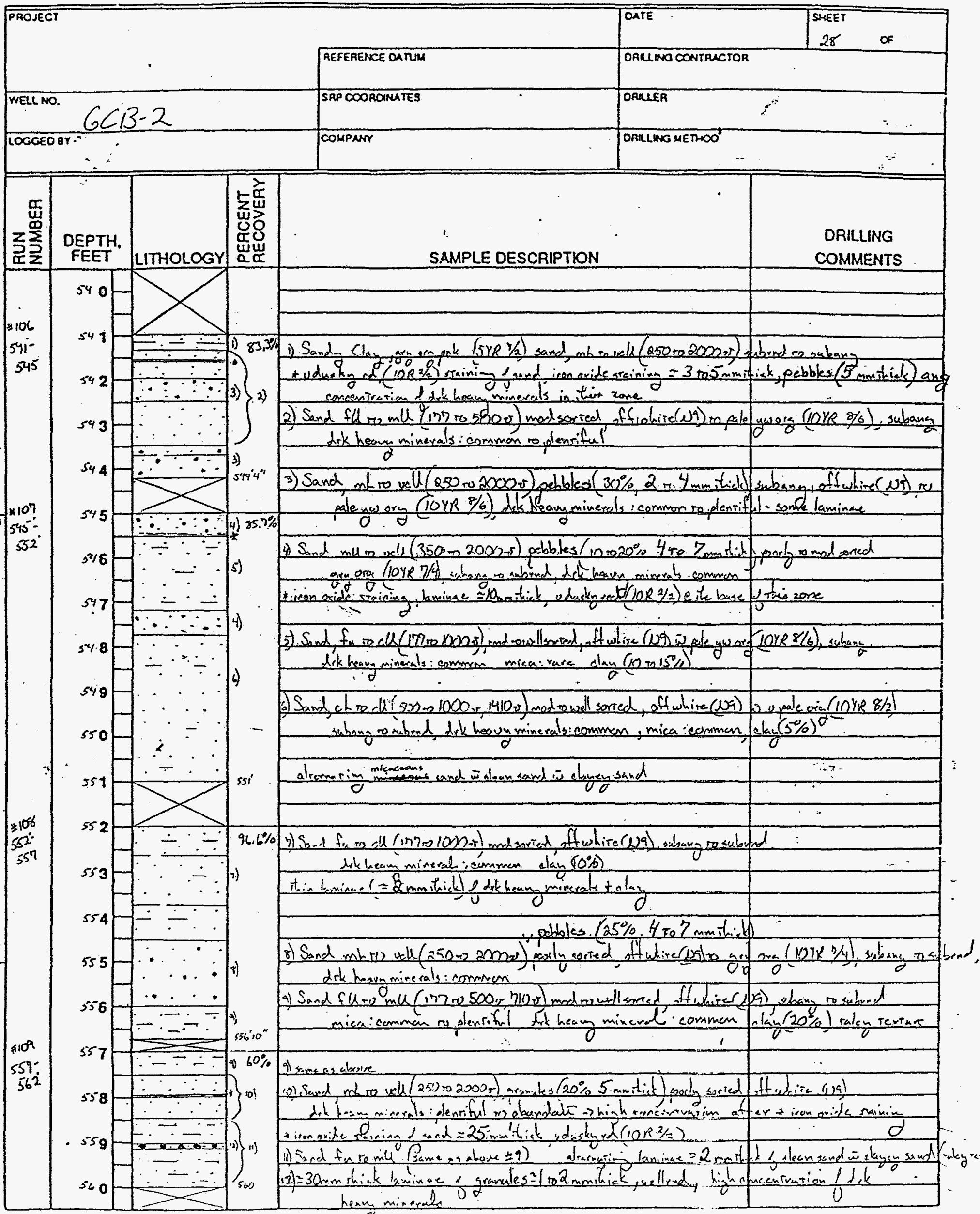




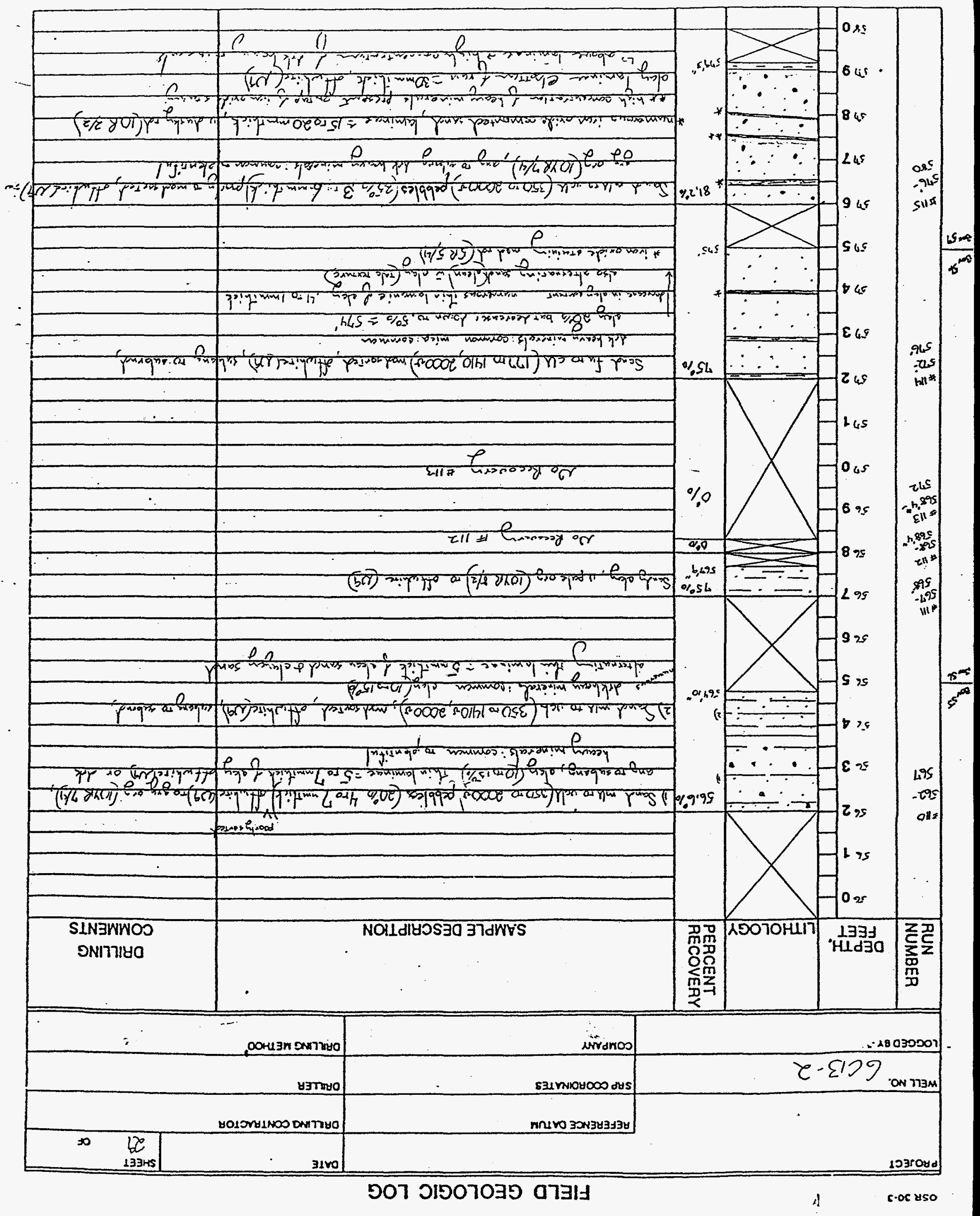


FIELD GEOLOGIC LOG

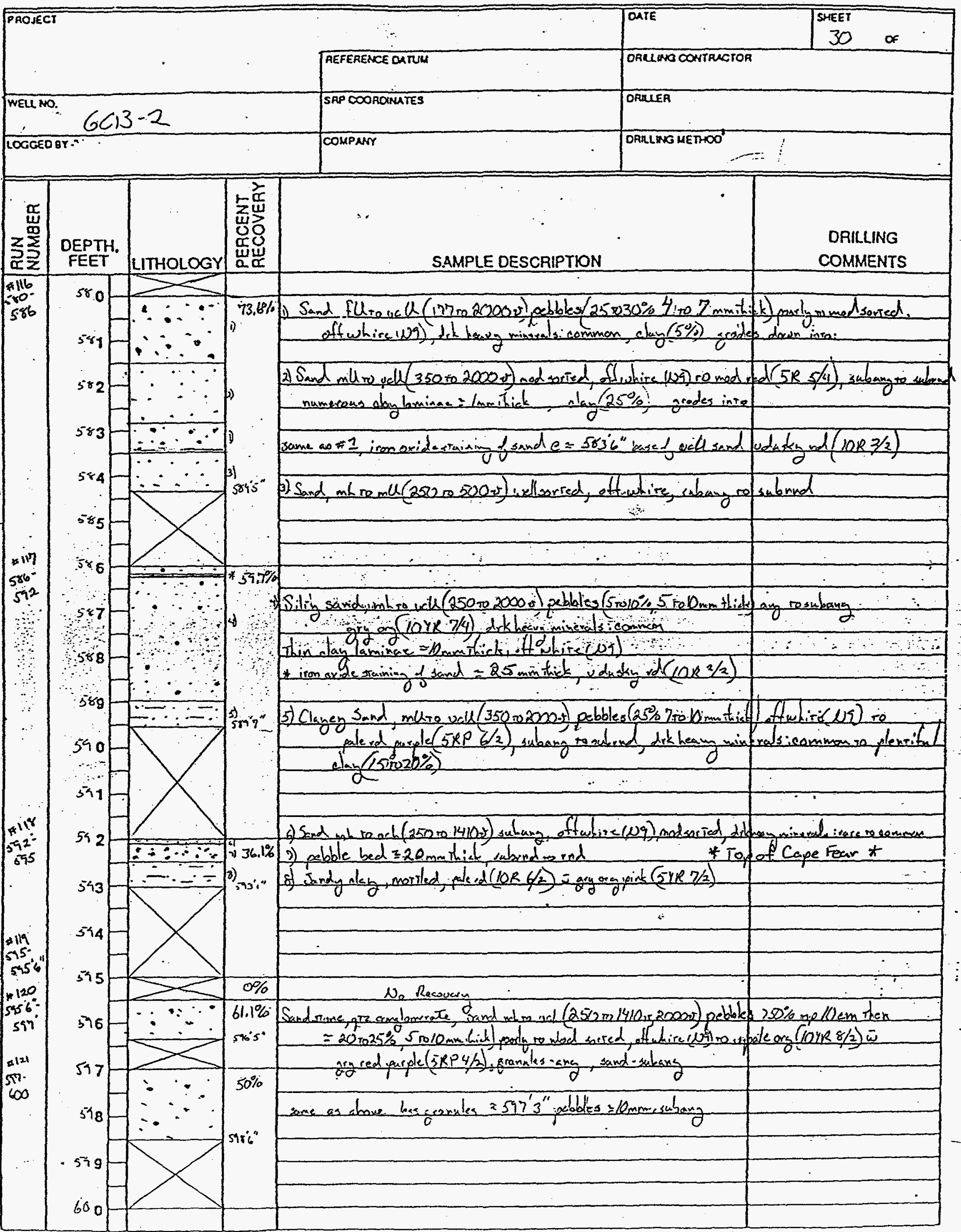




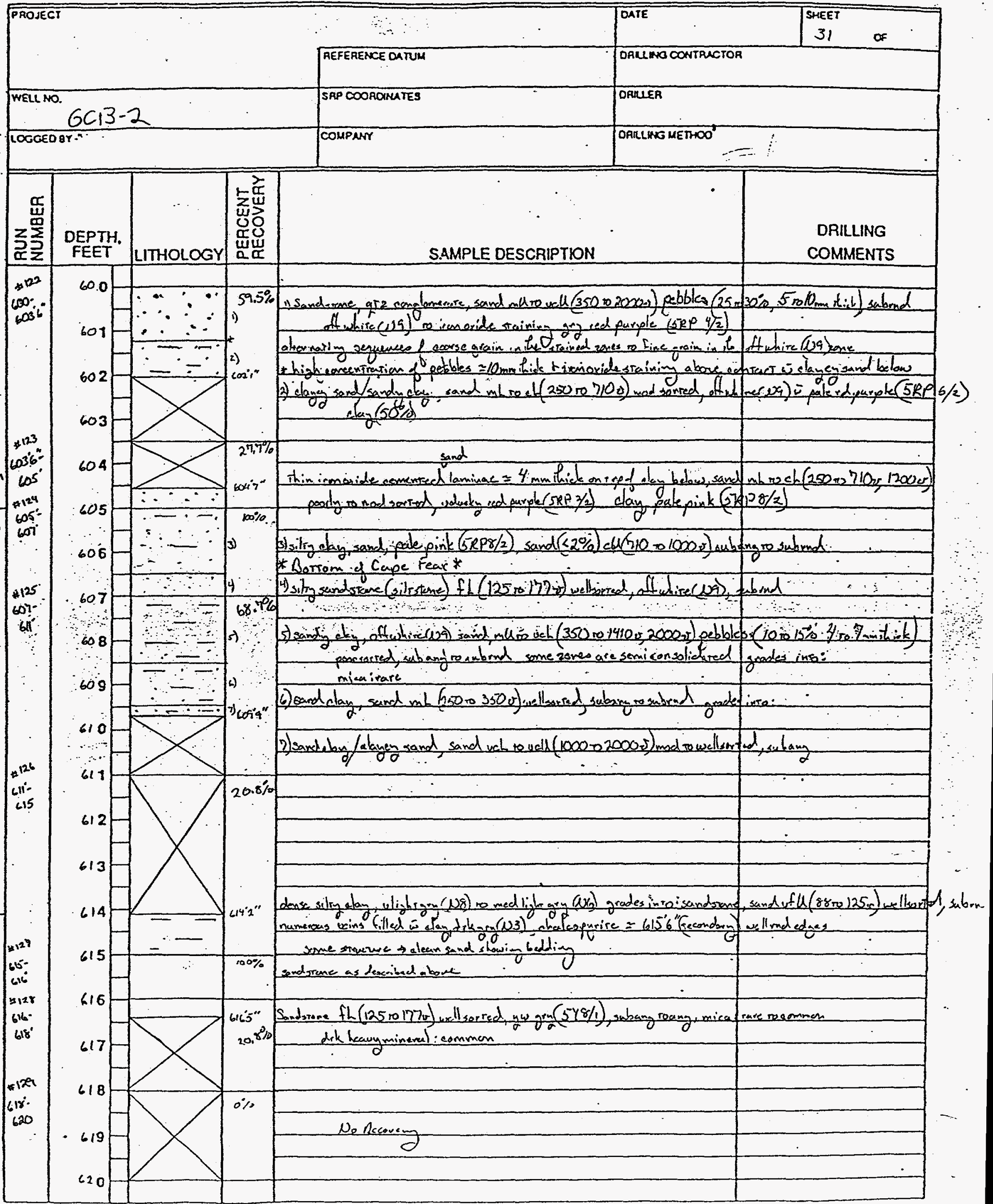




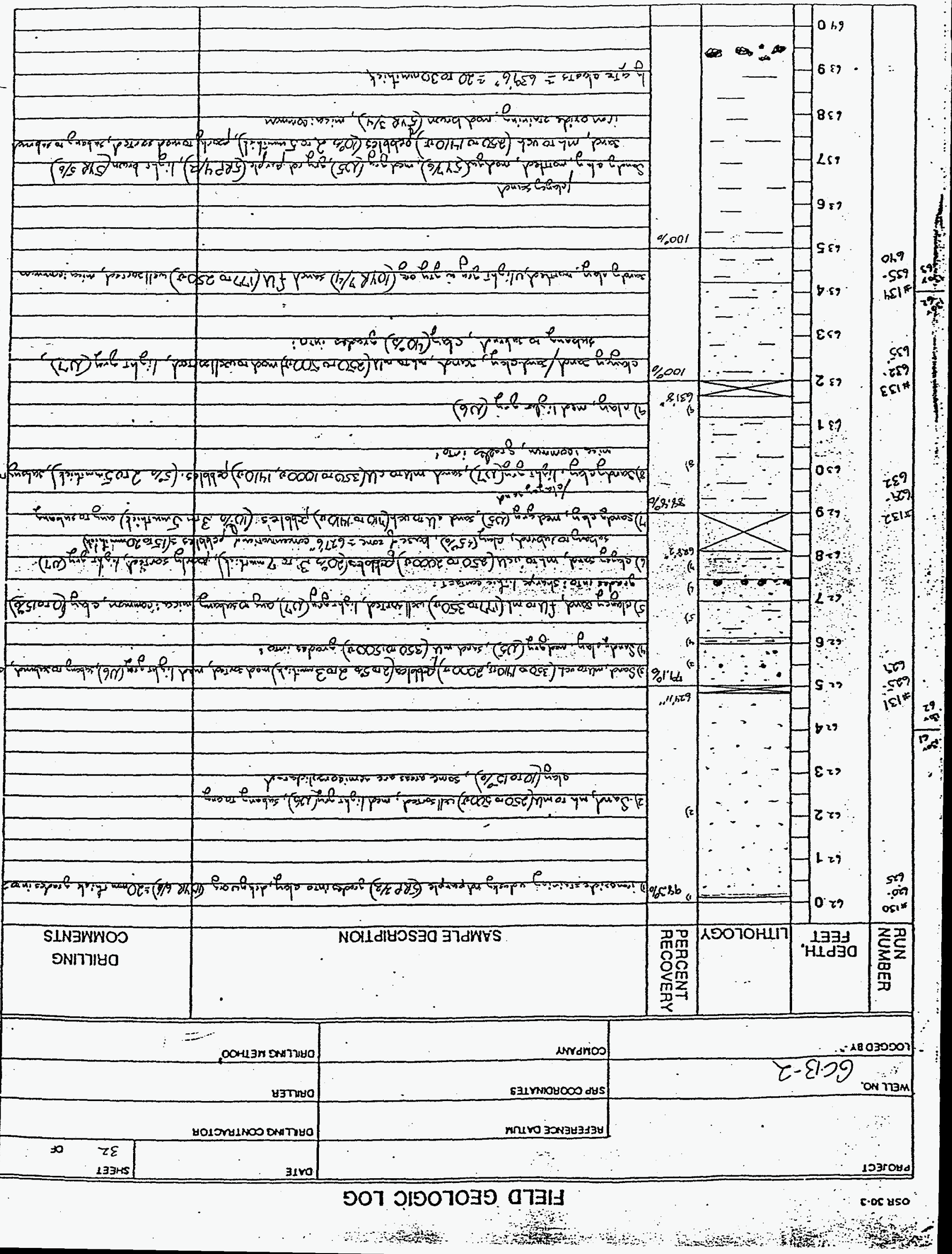


FIELD GEOLOGIC LOG

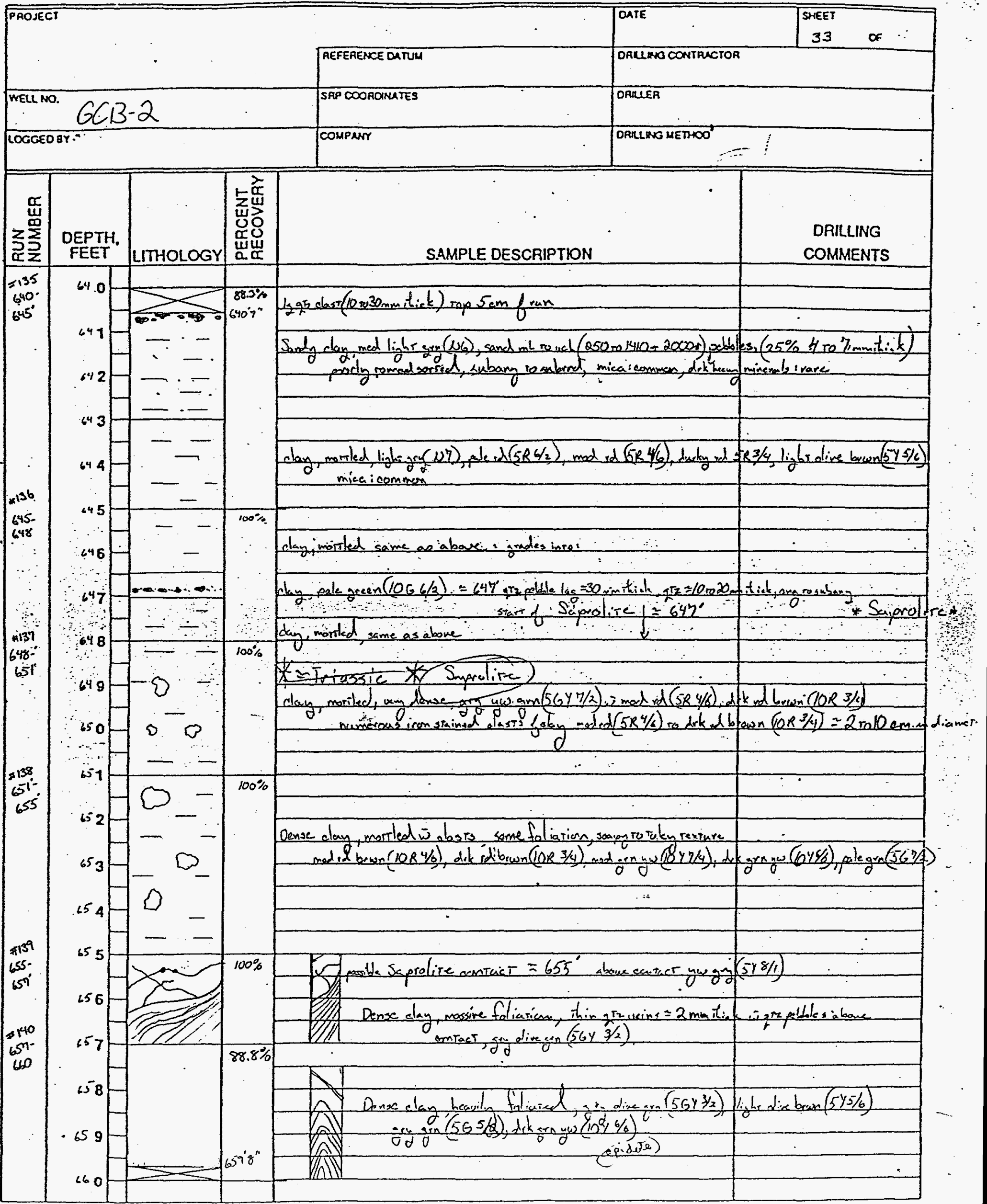




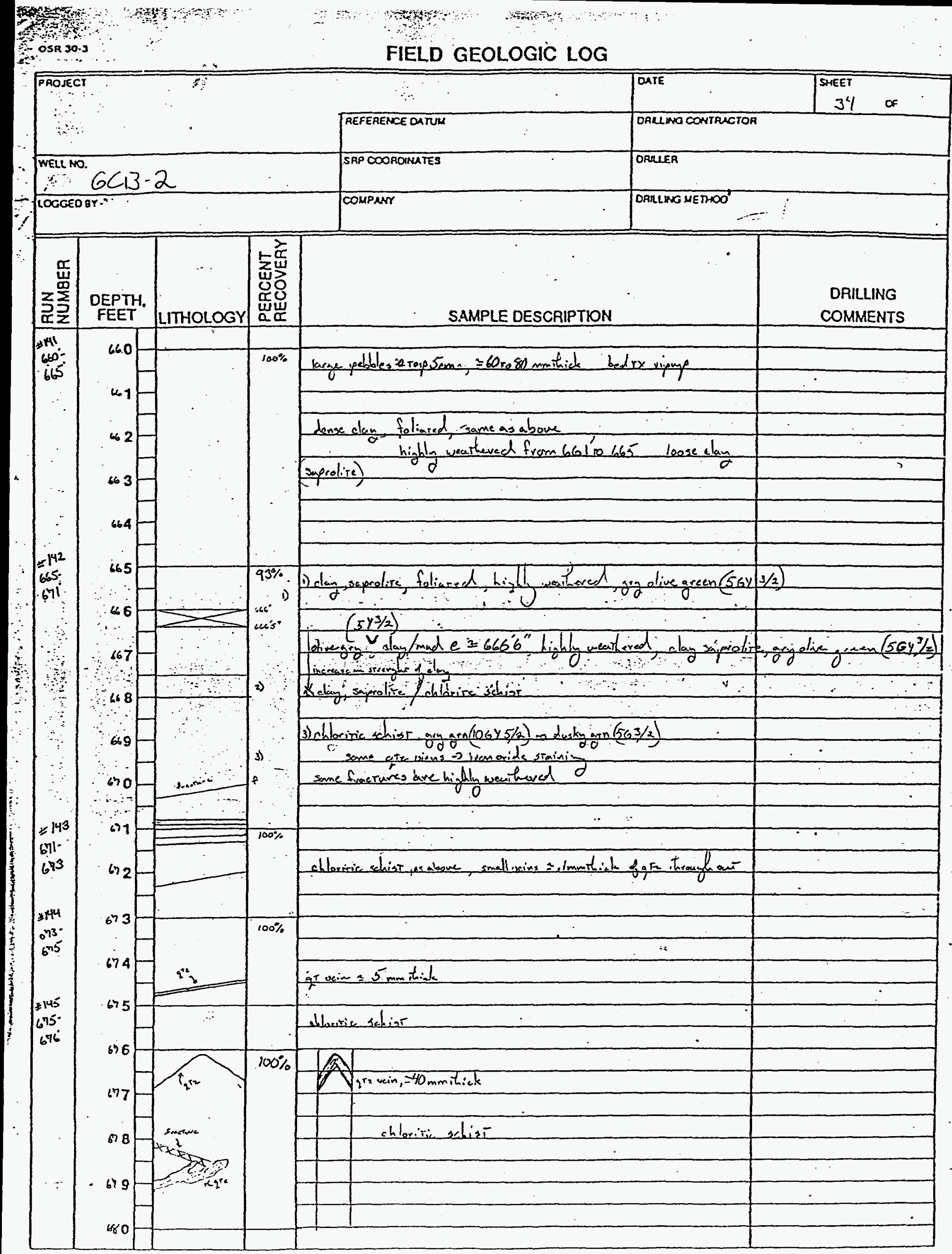




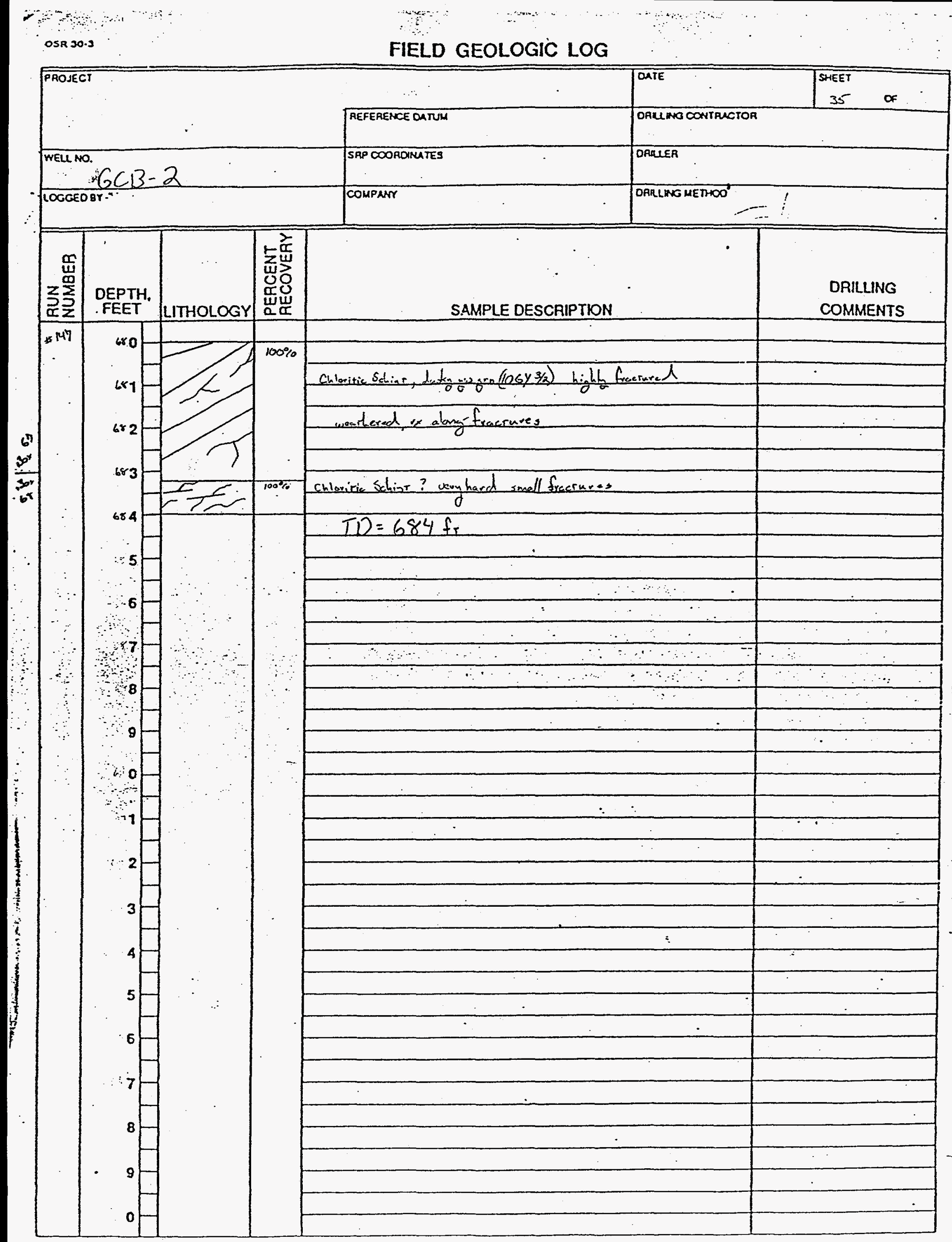


Core-Hole: \# GCB-3

7-1-96

Rotary down to $11^{\prime} 10^{\prime \prime}$

\begin{tabular}{|c|c|c|c|}
\hline Run 1 & $11^{\prime} 10^{\prime \prime}-15^{\prime}$ & \multicolumn{2}{|l|}{$100 \%$} \\
\hline Run 2 & $15-18$ & $2^{\prime} 3^{\prime \prime}$ & $15-17^{\prime \prime} 3^{\prime \prime}$ \\
\hline Run 3 & $18-25^{\prime}$ & $5^{\prime} 9^{\prime \prime}$ & $18-23^{\prime} 9^{\prime \prime}$ \\
\hline Run 4 & $25-31$ & $4^{\prime} 6^{\prime \prime}$ & $25-29^{\prime} 6^{\prime \prime}$ \\
\hline Run 5 & $31-35$ & $3^{\prime} 8^{\prime \prime}$ & $31-34^{\prime} 8^{\prime \prime}$ \\
\hline Run 6 & $35-40$ & $2^{\prime} 6^{\prime \prime}$ & $35-37^{\prime} 6^{\prime \prime}$ \\
\hline Run 7 & $40-45$ & $4^{\prime} 11^{\prime \prime}$ & $40-44^{\prime} 11^{\prime \prime}$ \\
\hline Run 8 & $45-46$ & $100 \%$ & \\
\hline Run 9 & $46-46.3$ & .25 & $46-46.25$ \\
\hline Run 10 & $46.3-47.3$ & .65 & $46.3-46.95$ \\
\hline Run 11 . & $47.3-49$ & $100 \%$ & \\
\hline Run 12 & $49-49.3$ & .2 & - 49-49.2 \\
\hline Run 13 & $49.3-52$ & 1.2 & $49.3-50.5$ \\
\hline Run 14 & $52-55$ & 1.7 & $52-53.7$ \\
\hline Run 15 & $55-59$ & 3.9 & $55-58.9$ \\
\hline Run 16 & $59-65$ & 5.4 & $59-64.4$ \\
\hline Run 17 & $65-72$ & 6.1 & $65-71.1$ \\
\hline Run 18 & $72-79$ & 6.4 & $72-78.4$ \\
\hline Run 19 & $79-83$ & 3.4 & $79-82: 4$ \\
\hline Run 20 & $83-87$ & 3.5 & $83-86.5$ \\
\hline Run 21 & $87-89$ & 1.6 & $87-88.6$ \\
\hline Run 22 & $89-90$ & .4 & $89-89.4$ \\
\hline Run 23 & $90-93$ & $100 \%$ & \\
\hline Run 24 & $93-99$ & $100 \%$ & \\
\hline$D_{3}$ & 9 & 1.2 & $99-100.2$ \\
\hline
\end{tabular}

7-2-96

Run 26

Run 27

102-105

$100 \%$

$2^{\prime} \quad 105-107$

Run 28

105-108

$.7 \quad 108-108.7$

Run $29 \quad 112-115$

Run 30

$115-116$

Run 31

$116-118$

Run 32

$118-120$

Run 33

$120-123$

Run 34

123-124

Run 35

124-126

Run 36

126-126.5

Run 37

$126.5-128$

Run 38

128-131

$2 \quad 112-114$

no recovery

$1.7 \quad 116-117.7$

$.5 \quad 118-118.5$

$1.4 \quad 120-121.4$

no recovery

$1.4 \quad 124-125.4$

$.3 \quad 126-126.3$

$.9 \quad 126-127.4$

$2 \quad 128-130$ 


\begin{tabular}{|c|c|c|c|}
\hline Run 39 & $131-134$ & 2 & $131-133$ \\
\hline Run 40 & $134-138$ & $100 \%$ & \\
\hline Run 41 & $138-143$ & 4.6 & $138-142.6$ \\
\hline Run 42 & $143-148$ & 4.8 & $143-147.8$ \\
\hline Run 43 & $148-153$ & $100 \%$ & \\
\hline Run 44 & $153-155$ & 1.5 & $153-154.5$ \\
\hline Run 45 & $155-158$ & $100 \%$ & \\
\hline Run 45a & $158-160$ & $100 \%$ & \\
\hline Run 46 & $160-164$ & $100 \%$ & \\
\hline Run 47 & $164-169$ & 4.6 & $164-168.6$ \\
\hline Run 48 & $169-173$ & no rec & very \\
\hline Run 49 & $173-176$ & $100 \%$ & \\
\hline Run 50 & $176-180$ & 3.8 & $176-179.8$ \\
\hline Run 51 & $180-185$ & $100 \%$ & \\
\hline Run 52 & $185-192$ & 5.9 & $185-190.9$ \\
\hline Run 53 & $192-197$ & 4.7 & $192-196.7$ \\
\hline Run 54 & $197-202$ & $100 \%$ & \\
\hline Run 55 & $202-206$ & $100 \%$ & \\
\hline Run 56 & $206-213$ & $100 \%$ & \\
\hline $7-11-96$ & & & \\
\hline Run 57 & $213-217$ & 3.3 & $213-216.3$ \\
\hline Run 58 & $217-220$ & $100 \%$ & \\
\hline Run 59 & $220-225$ & 4.8 & $220-224.8$ \\
\hline Run 60 & $225-232$ & 6.3 & $225-231.3$ \\
\hline Run 61 & $232-237$ & $100 \%$ & \\
\hline Run 62 & $237-245$ & $100 \%$ & \\
\hline Run 63 & $245-253$ & $100 \%$ & \\
\hline Run 64 & $253-258$ & $100 \%$ & \\
\hline Run 65 & $258-265$ & 6.7 & $258-264.7$ \\
\hline Run 66 & $265-273$ & 7.8 & $265-272.8$ \\
\hline Run 67 & $273-275$ & \multicolumn{2}{|c|}{ no recovery } \\
\hline Run 68 & $275-276$ & \multicolumn{2}{|c|}{ no recovery } \\
\hline Run 69 & $276-280$ & 3.8 & $276-279.8$ \\
\hline Run 70 & $280-285$ & 4.9 & $280-284.9$ \\
\hline Run 71 & $285-287$ & 1.7 & $285-286.7$ \\
\hline Run 72 & $287-292$ & 4.5 & $287-291.5$ \\
\hline \multicolumn{4}{|l|}{$7-13-96$} \\
\hline Run 73 & $292-295$ & $100 \%$ & \\
\hline Run 74 & $295-300$ & 3.8 & $295-298.8$ \\
\hline Run 75 & $300-303$ & 1.3 & $300-301.3$ \\
\hline Run 76 & $303-306$ & 1.7 & $303-304.7$ \\
\hline Run 77 & $306-309$ & $100 \%$ & \\
\hline
\end{tabular}




\begin{tabular}{|c|c|c|c|}
\hline & & $\underline{\operatorname{Res}}$ & overed \\
\hline Run 78 & $309-310$ & .5 & $309-309.5$ \\
\hline Run 79 & $310-312$ & .3 & $310-310.3$ \\
\hline Run 80 & $312-315$ & 2.8 & $312-314.8$ \\
\hline Run 81 & $315-319$ & 3.6 & $315-318.6$ \\
\hline Run 82 & $319-320$ & .3 & $319-319.3$ \\
\hline Run 83 & $320-324$ & $100 \%$ & \\
\hline Run 84 & $324-331$ & 6.2 & $324-330.2$ \\
\hline Run 85 & $331-334$ & 2.8 & $331-333.8$ \\
\hline Run 86 & $334-337$ & 2.9 & $334-336.9$ \\
\hline Run 87 & $337-341$ & 3.6 & $337-340.6$ \\
\hline Run 88 & $341-346$ & 4.9 & $341-345.9$ \\
\hline Run 89 & $346-353$ & 6.8 & $346-352.8$ \\
\hline $7-14-96$ & & & \\
\hline Run 90 & $353-358$ & 4 & $353-357$ \\
\hline Run 91 & $358-361$ & 2.2 & $258-260.2$ \\
\hline Run 92 & $361-363$ & 1.7 & $361-362.7$ \\
\hline Run 93 & $363-365$ & $100 \%$ & \\
\hline Run 94 & $365-372$ & 6.4 & $365-371.4$ \\
\hline Run 95 & $372-376$ & 2.6 & $372-374.6$ \\
\hline Run 96 & $376-380$ & 3.3 & $376-379.3$ \\
\hline Run 97 & $380-383$ & 2.1 & $380-382.1$ \\
\hline Run 98 & $383-387$ & 3.7 & $383-386.7$ \\
\hline Run 99 & $387-388$ & no rec & overy \\
\hline Run 100 & $388-392$ & no rec & overy \\
\hline Run 101 & $392-392.7$ & $100 \%$ & \\
\hline Run 102 & $392.7-395$ & 1.5 & $392.7-394.2$ \\
\hline Run 103 & $395-399$ & 1.6 & $395-396.6$ \\
\hline Run 104 & $399-401$ & 1.9 & $399-400.9$ \\
\hline Run 105 & $401-404$ & 2.9 & $401-403.9$ \\
\hline Run 106 & $404-407$ & $100 \%$ & \\
\hline Run 107 & $407-408$ & .4 & $407-407.4$ \\
\hline Run 108 & $408-411.2$ & 1.5 & $408-409.5$ \\
\hline $7-15-96$ & & & \\
\hline Run 109 & $411.2-411.5$ & $100 \%$ & \\
\hline Run 110 & $411.5-412.5$ & $100 \%$ & \\
\hline Run 111 & $412.5-416$ & 3.2 & $412.5-415.7$ \\
\hline Run 112 & $416-420$ & 3.7 & $416-419.7$ \\
\hline $7-16-96$ & & & \\
\hline Run 113 & $420-425$ & 2.6 & $420-422.6$ \\
\hline Run 114 & $425-427$ & 1.7 & $425-426.7$ \\
\hline Run 115 & $427-430$ & 2 & $427-429$ \\
\hline
\end{tabular}




\begin{tabular}{|c|c|c|c|}
\hline Run 116 & $430-433$ & 2.9 & $430-432.9$ \\
\hline Run 117 & $433-436$ & 2.4 & $433-435.4$ \\
\hline Run 118 & $436-438$ & 1.6 & $436-437.6$ \\
\hline Run 119 & $438-441$ & 2.5 & $438-440.5$ \\
\hline Run 120 & $441-445$ & 3.6 & $441-444.6$ \\
\hline Run 121 & $445-449$ & 2.6 & $445-447.6$ \\
\hline Run 122 & $449-453$ & 1.1 & $449-450.1$ \\
\hline Run 123 & $453-455$ & 1.6 & $453-454.6$ \\
\hline Run 124 & $455-458$ & 2.5 & $455-457.5$ \\
\hline Run 125 & $458-461$ & 1.7 & $458-459.7$ \\
\hline Run 126 & $461-464$ & 2.3 & $461-463.3$ \\
\hline Run 127 & $464-469$ & 3.5 & $464-467.5$ \\
\hline Run 128 & $469-473$ & 3.3 & $469-472.3$ \\
\hline Run 129 & $473-476$ & 2.1 & $473-475.1$ \\
\hline Run 130 & $476-480$ & 3.2 & $476-479.2$ \\
\hline Run 131 & $480-485$ & 3.7 & $480-483.7$ \\
\hline Run 132 & $485-485.4$ & $100 \%$ & \\
\hline Run 133 & $485.4-492$ & 4.7 & $485.4-490.1$ \\
\hline $7-20-96$ & & & \\
\hline Run 134 & $492-493.2$ & .8 & $492-492.8$ \\
\hline Run 135 & $493.2-495$ & .2 & $493.2-493.4$ \\
\hline Run 136 & $495-498$ & 2.6 & $495-497.6$ \\
\hline Run 137 & $498-499.5$ & 1.4 & $498-499.4$ \\
\hline Run 138 & $499.5-503$ & 2.8 & $499.5-502.3$ \\
\hline Run 139 & $503-503.5$ & .2 & $503-503.2$ \\
\hline Run 140 & $503.5-505$ & .3 & $503.5-503.8$ \\
\hline Run 141 & $505-509$ & \multicolumn{2}{|c|}{ no recovery } \\
\hline Run 142 & $509-511$ & 1.3 & $509-510.3$ \\
\hline Run 143 & $511-512$ & .5 & $511-511.5$ \\
\hline Run 144 & $512-515$ & 2.5 & $512-514.5$ \\
\hline Run 145 & $515-519$ & 2.5 & $515-517.5$ \\
\hline Run 146 & $519-523$ & 3.8 & $519-522.8$ \\
\hline Run 147 & $523-527$ & 3.2 & $523-526.2$ \\
\hline Run 148 & $527-532$ & 4.2 & $527-531.2$ \\
\hline Run 149 & $532-536$ & 2.9 & $532-534.9$ \\
\hline Run 150 & $536-540$ & 3.1 & $536-539.1$ \\
\hline Run 151 & $540-542$ & 1.2 & $540-541.2$ \\
\hline Run 152 & $542-547$ & 3 & $542-545$ \\
\hline \multicolumn{4}{|l|}{$7-21-96$} \\
\hline Run 153 & $547-552.5$ & 4.5 & $547-551.5$ \\
\hline Run 154 & $552.5-558$ & 4.2 & $552.5-556.7$ \\
\hline Run 155 & $558-560$ & .6 & $558-558.6$ \\
\hline
\end{tabular}




\begin{tabular}{|c|c|c|c|}
\hline \multirow[b]{2}{*}{ Run 156} & \multirow[b]{2}{*}{$560-566$} & \multicolumn{2}{|c|}{ Recovered } \\
\hline & & 3.6 & $560-563.6$ \\
\hline Run 156a & $566-572$ & 4.3 & $566-570.3$ \\
\hline Run 157 & $572-576$ & 2.4 & $572-574.4$ \\
\hline Run 158 & $576-585$ & \multicolumn{2}{|c|}{ no recovery } \\
\hline Run 159 & $585-589$ & 3.9 & $585-588.9$ \\
\hline Run 160 & $589-592$ & 2.2 & Lost $590.2-591$ \\
\hline Run 161 & $592-595$ & $100 \%$ & \\
\hline Run 162 & $595-597.8$ & 2.5 & $595-597.5$ \\
\hline Run 163 & $597.8-601$ & $100 \%$ & \\
\hline Run 164 & $601-606$ & 4.9 & $601-605.9$ \\
\hline Run 165 & $606-612$ & 5.9 & $606-611.9$ \\
\hline Run $165 a$ & $612-616.3$ & $100 \%$ & \\
\hline Run $165 \mathrm{~b}$ & $616.3-620$ & $100 \%$ & \\
\hline Run 166 & $620-621$ & $100 \%$ & \\
\hline Run 167 & $621-628$ & 6.6 & $621-627.6$ \\
\hline Run 168 & $628-631$ & $100 \%$ & \\
\hline \multicolumn{4}{|l|}{$7-22-96$} \\
\hline Run 169 & $631-631.9$ & .6 & $631-631.6$ \\
\hline Run 170 & $631.9-632$ & $100 \%$ & \\
\hline
\end{tabular}


FIELD GEOLOGIC LOG

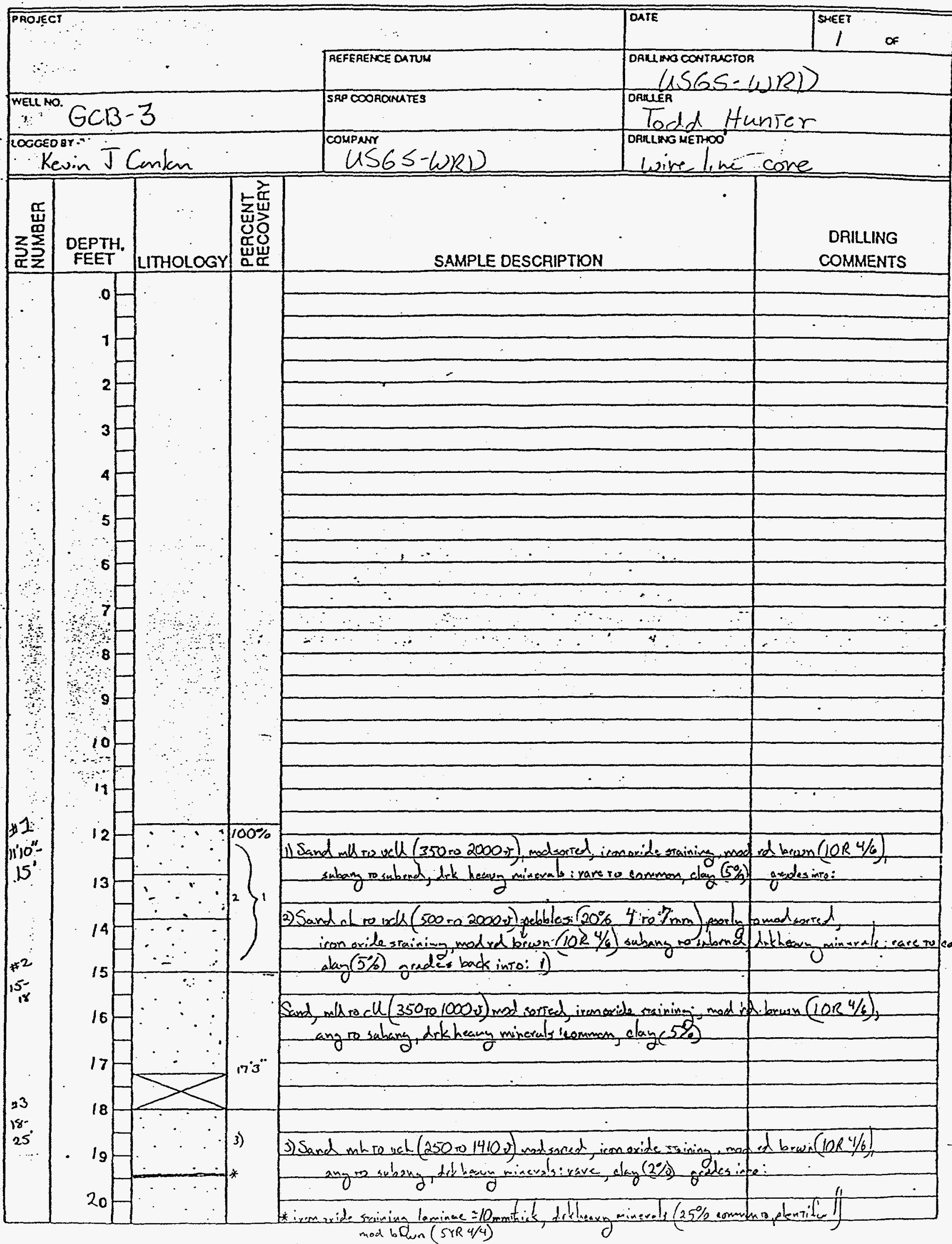




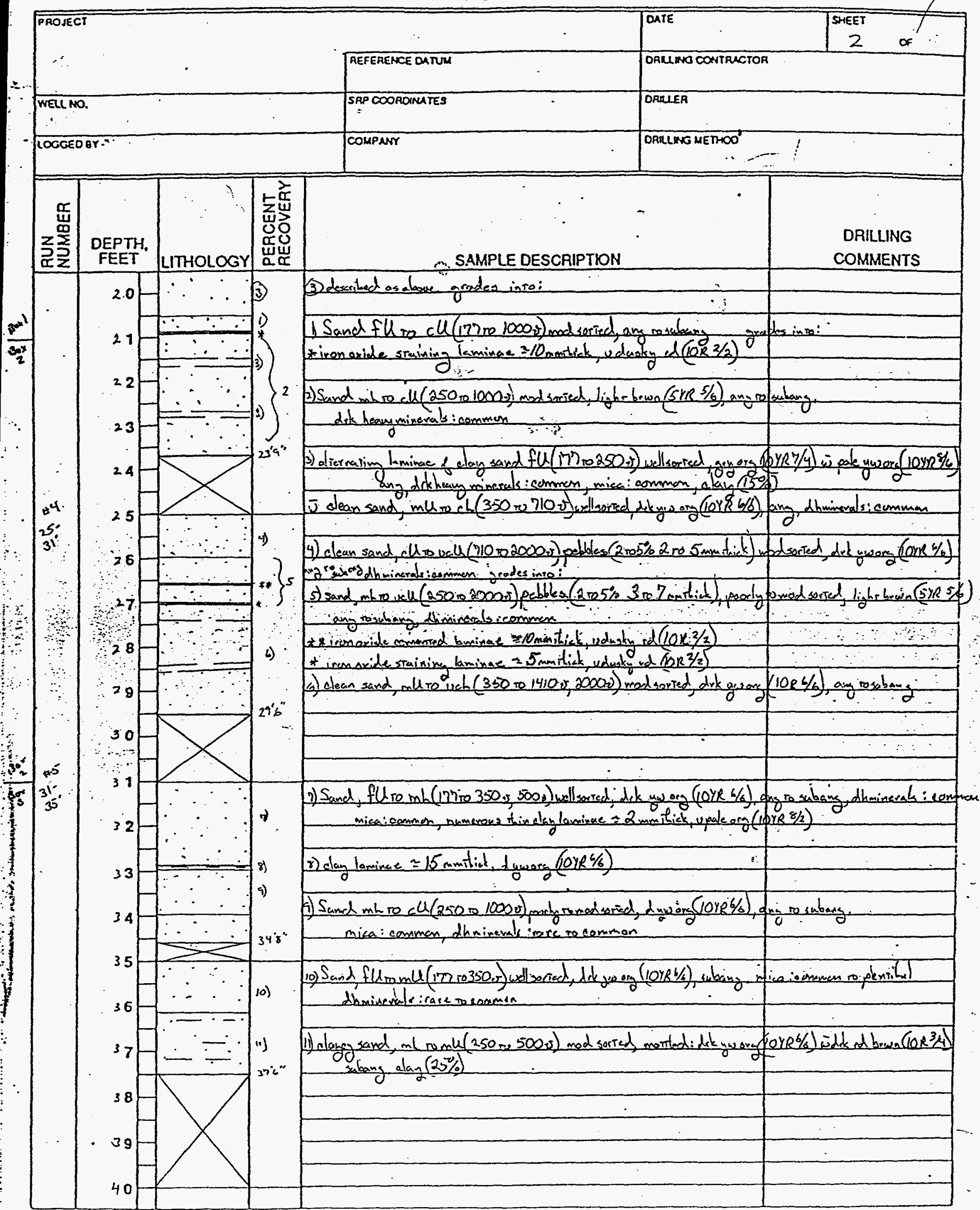




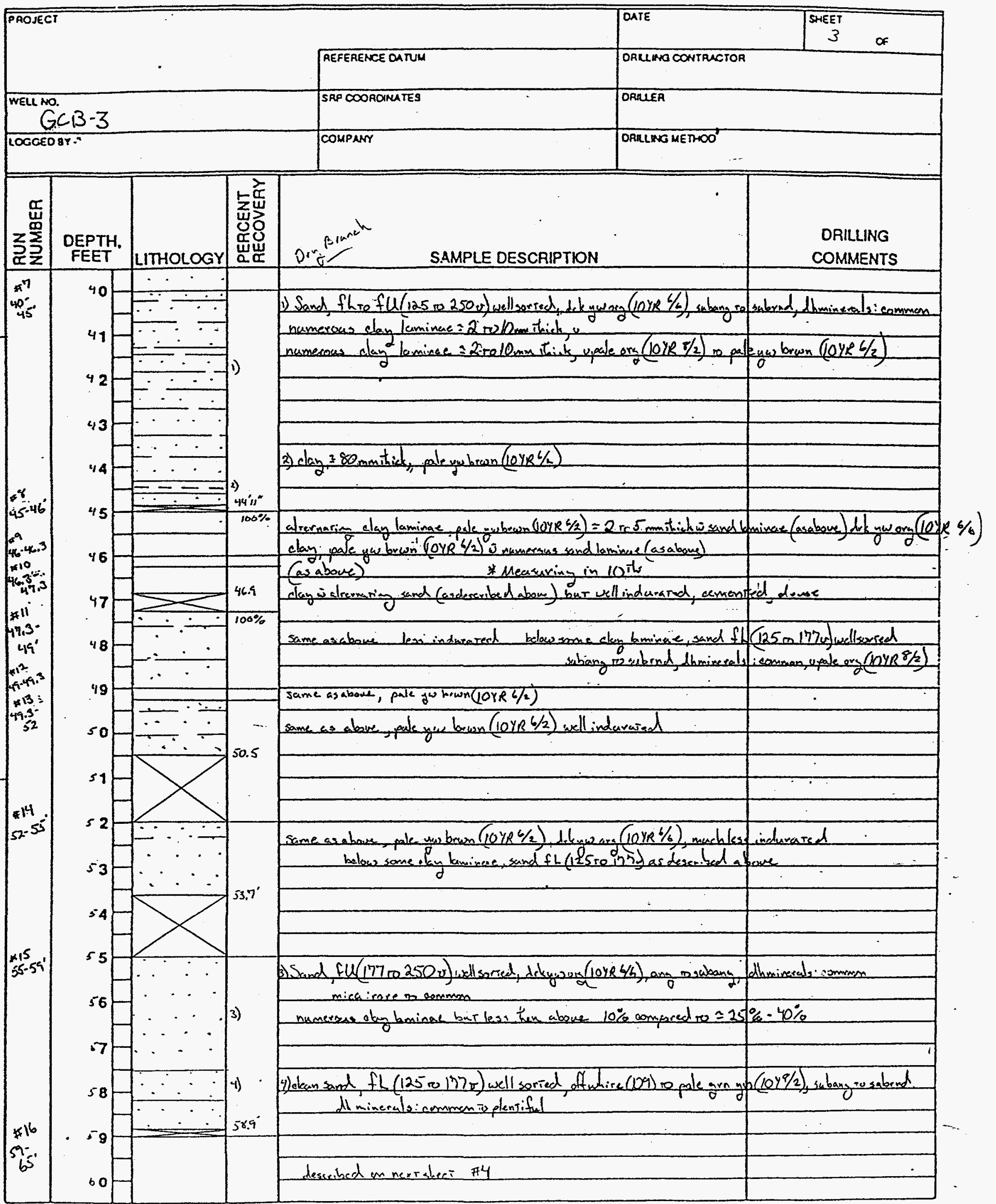


FIELD GEOLOGIC LOG

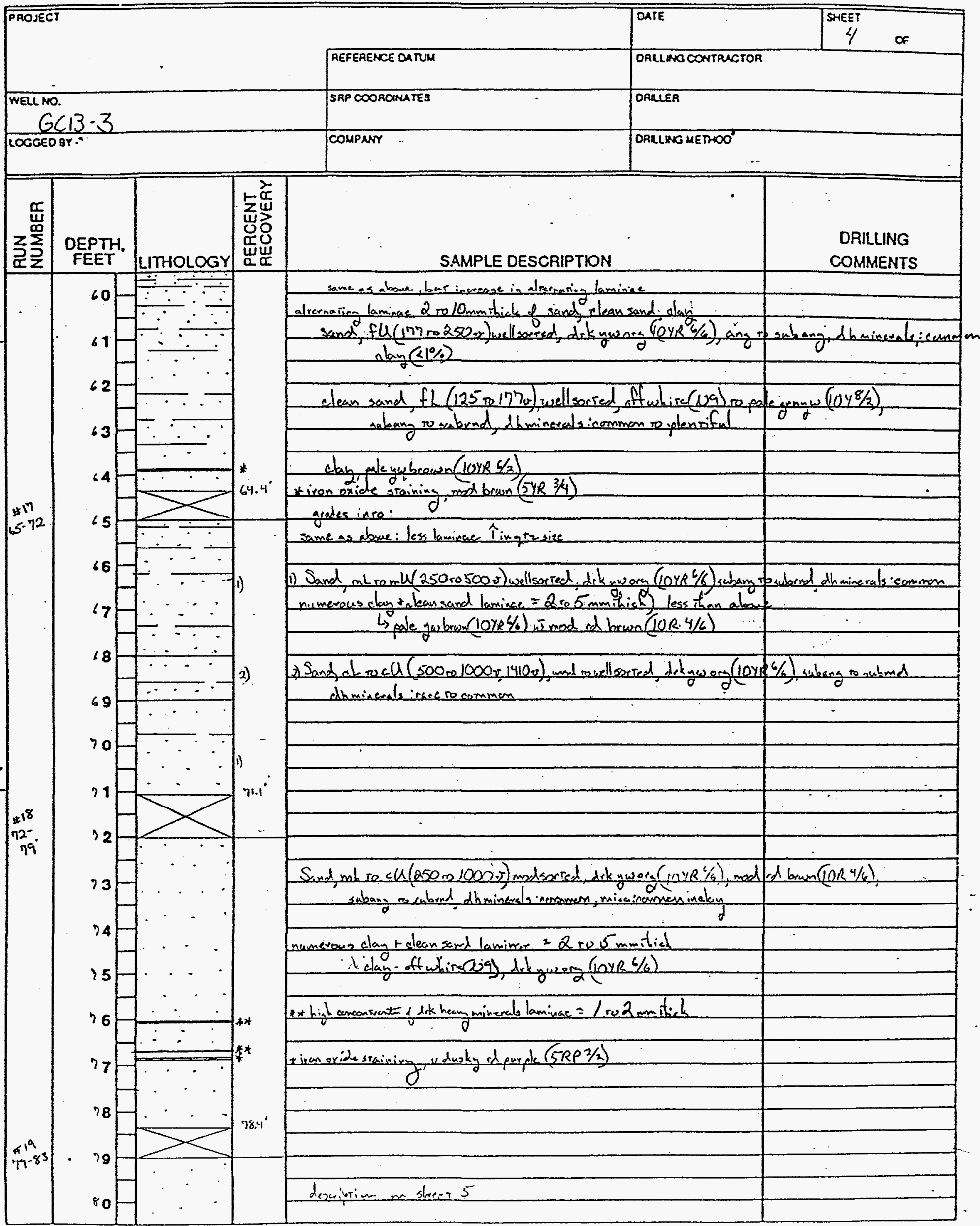


FIELD GEOLOGIC LOG

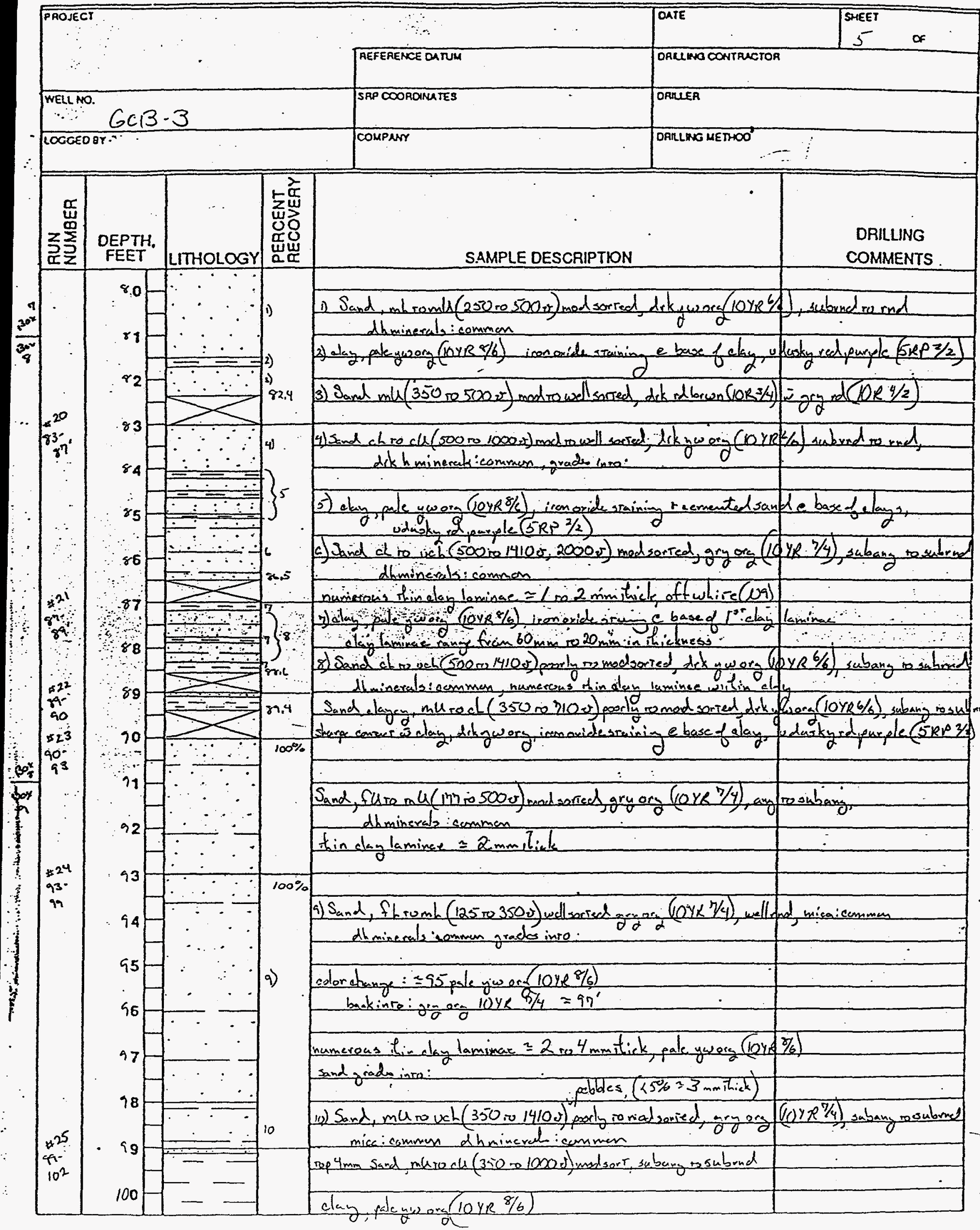


FIELD GEOLOGIC LOG

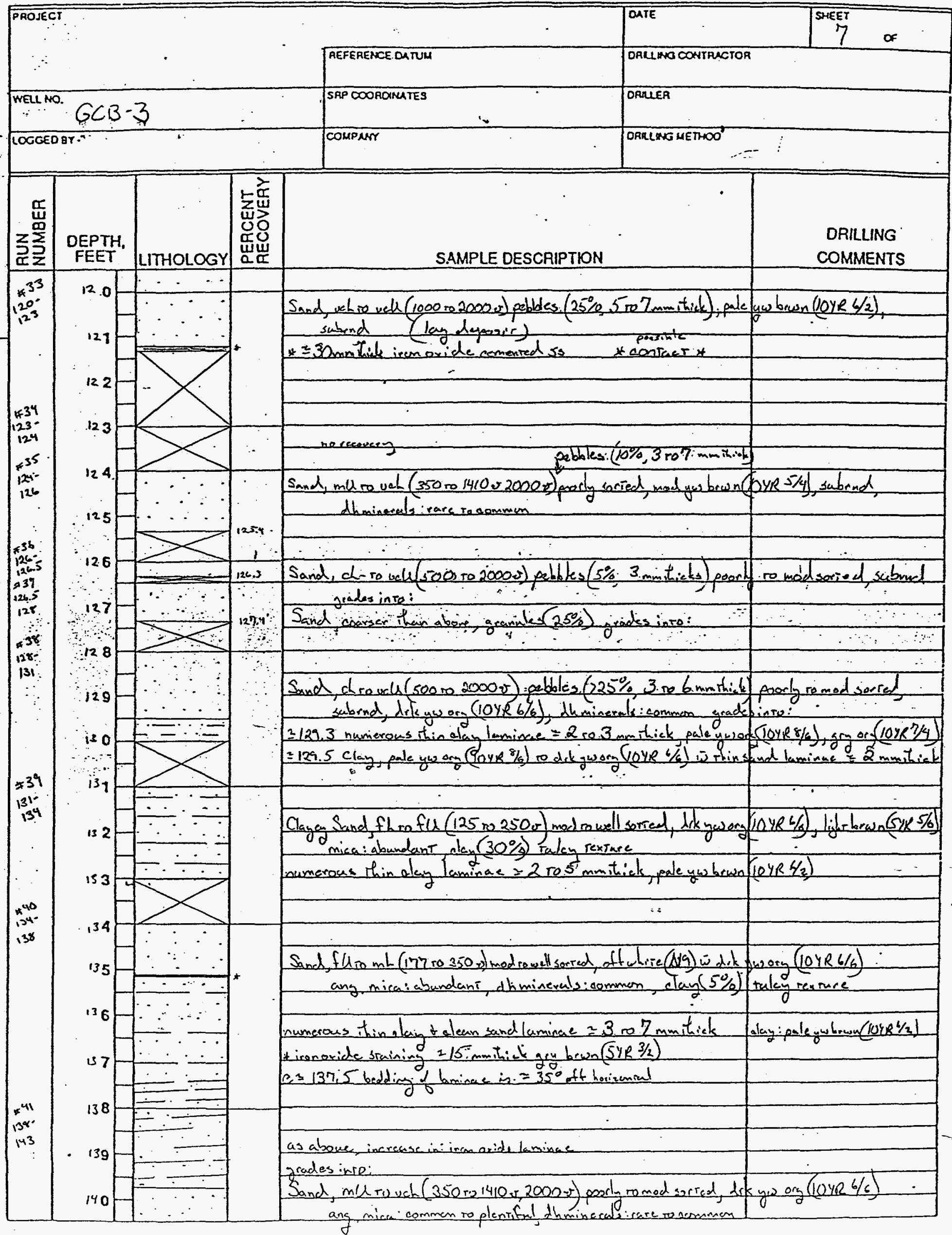




$$
\begin{array}{ll} 
& \\
\text { OSR 20.3 } & \text { FIELD GEOLOGIC LOG }
\end{array}
$$

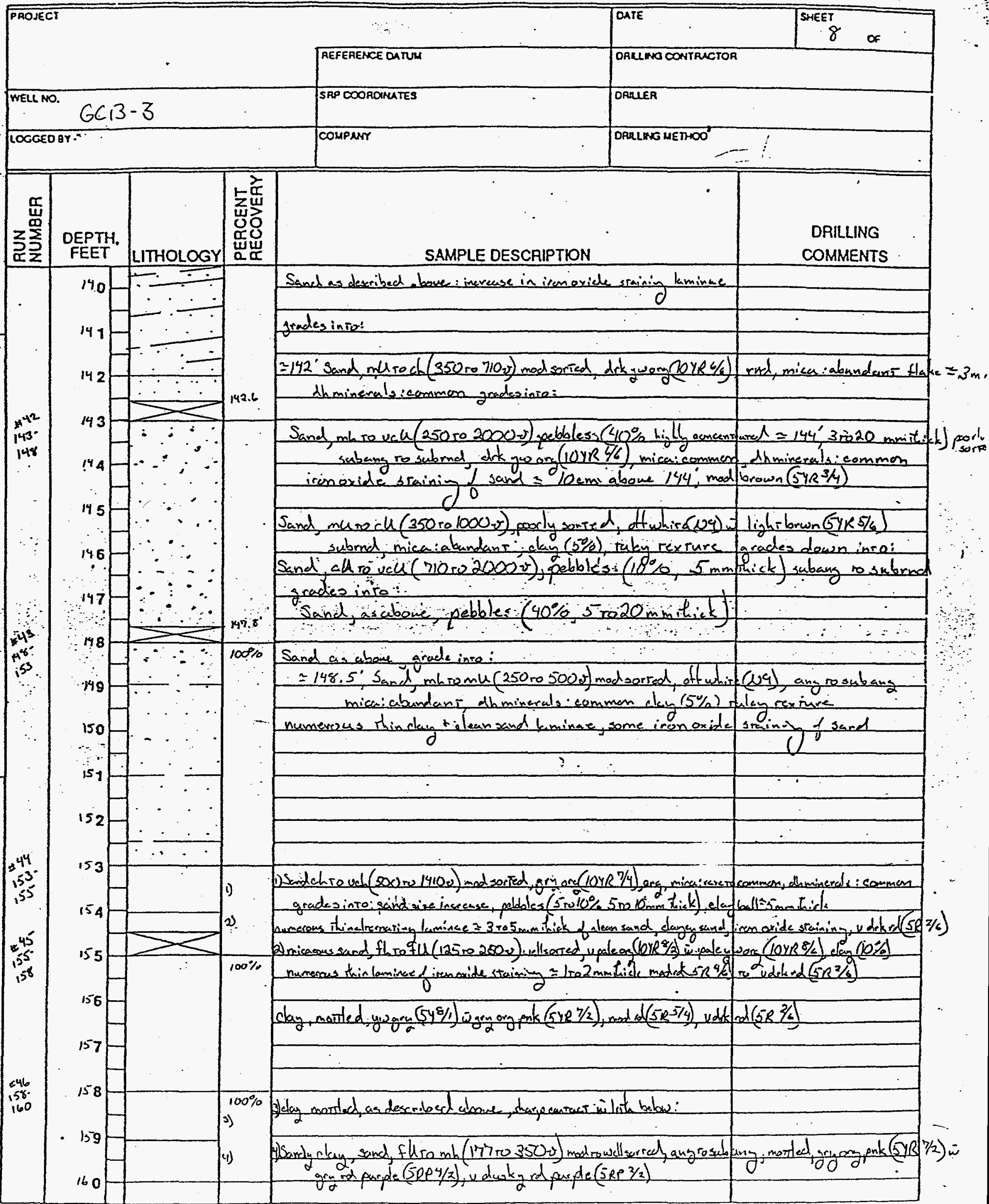




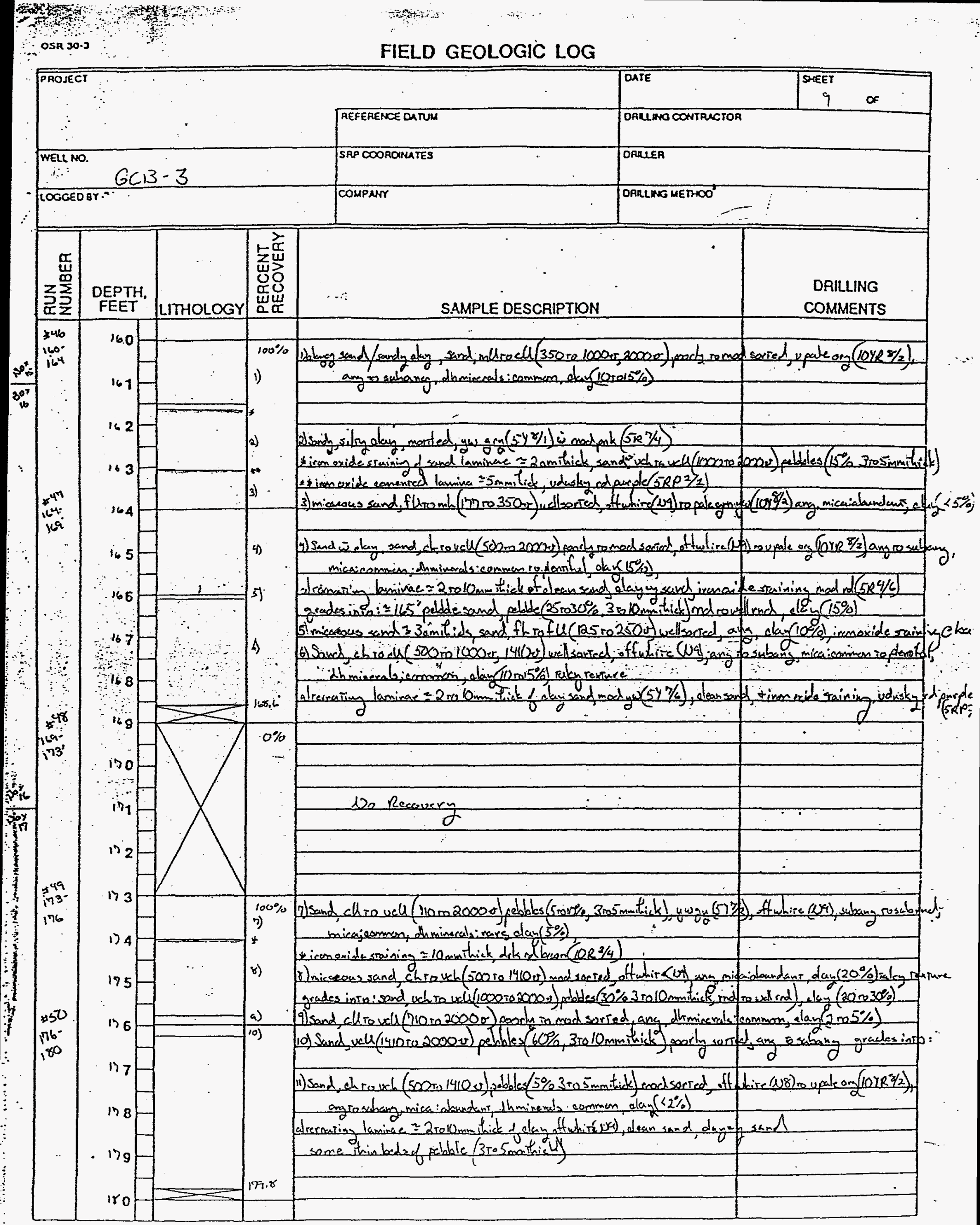




\section{FIELD GEOLOGIC LOG}

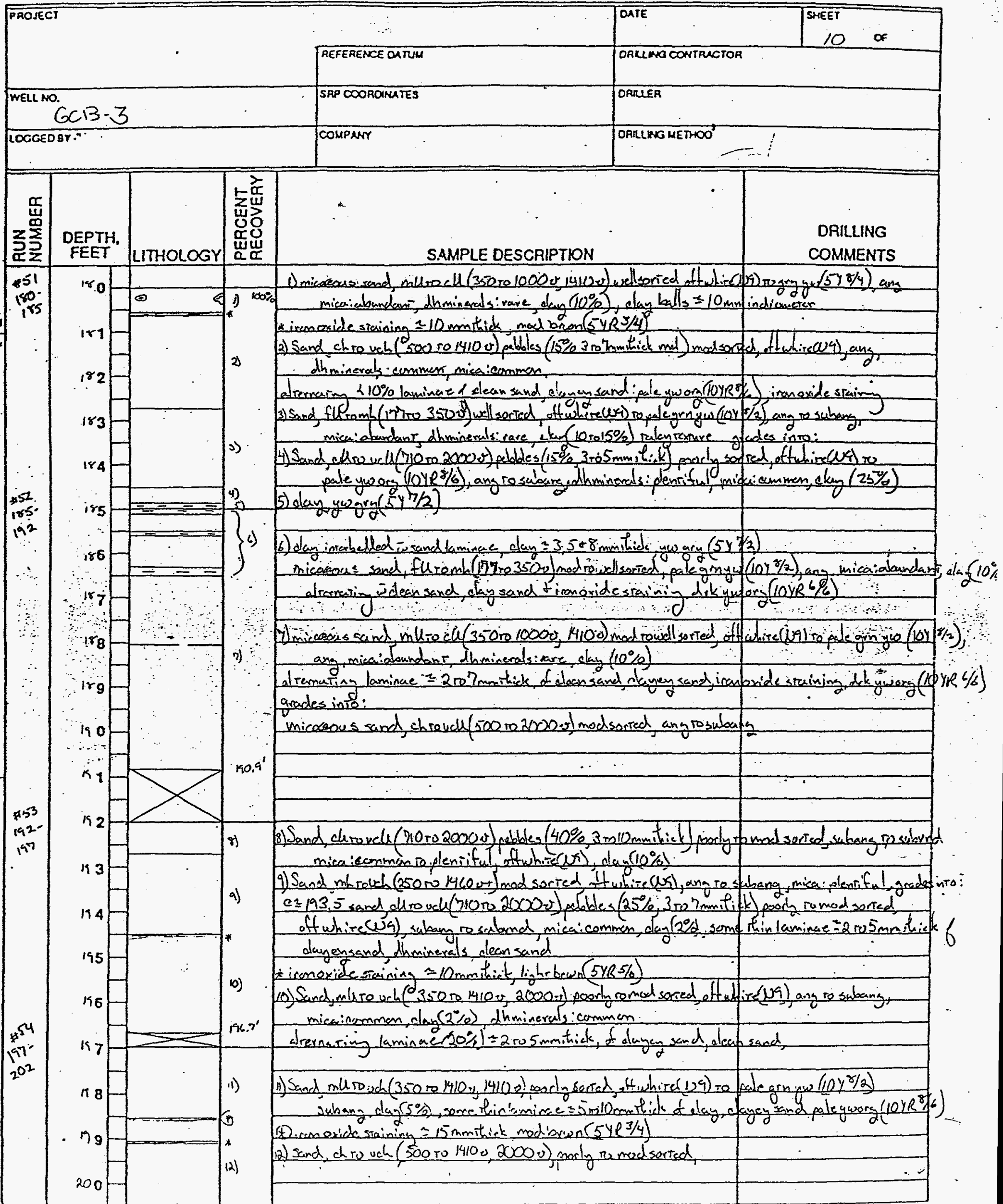




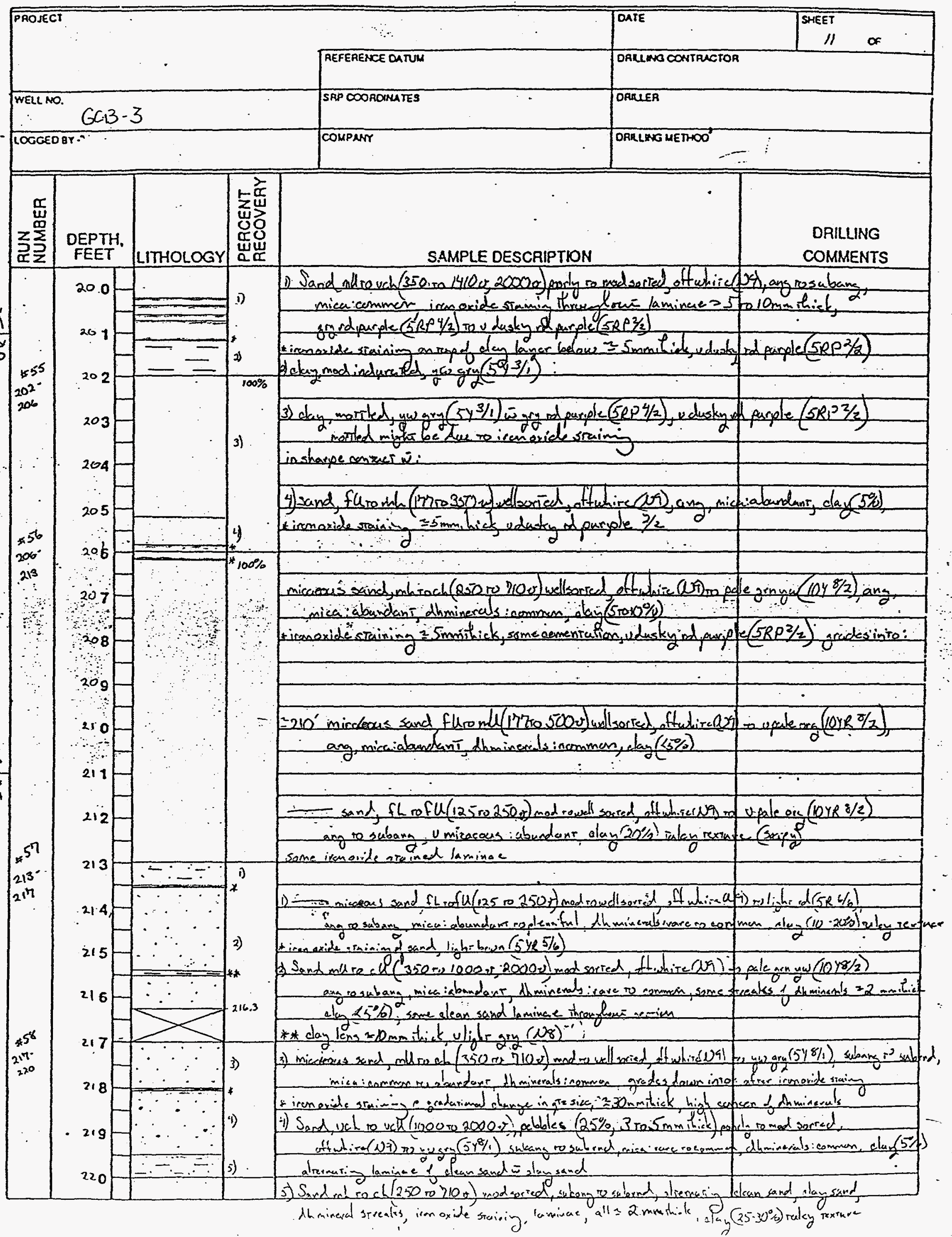




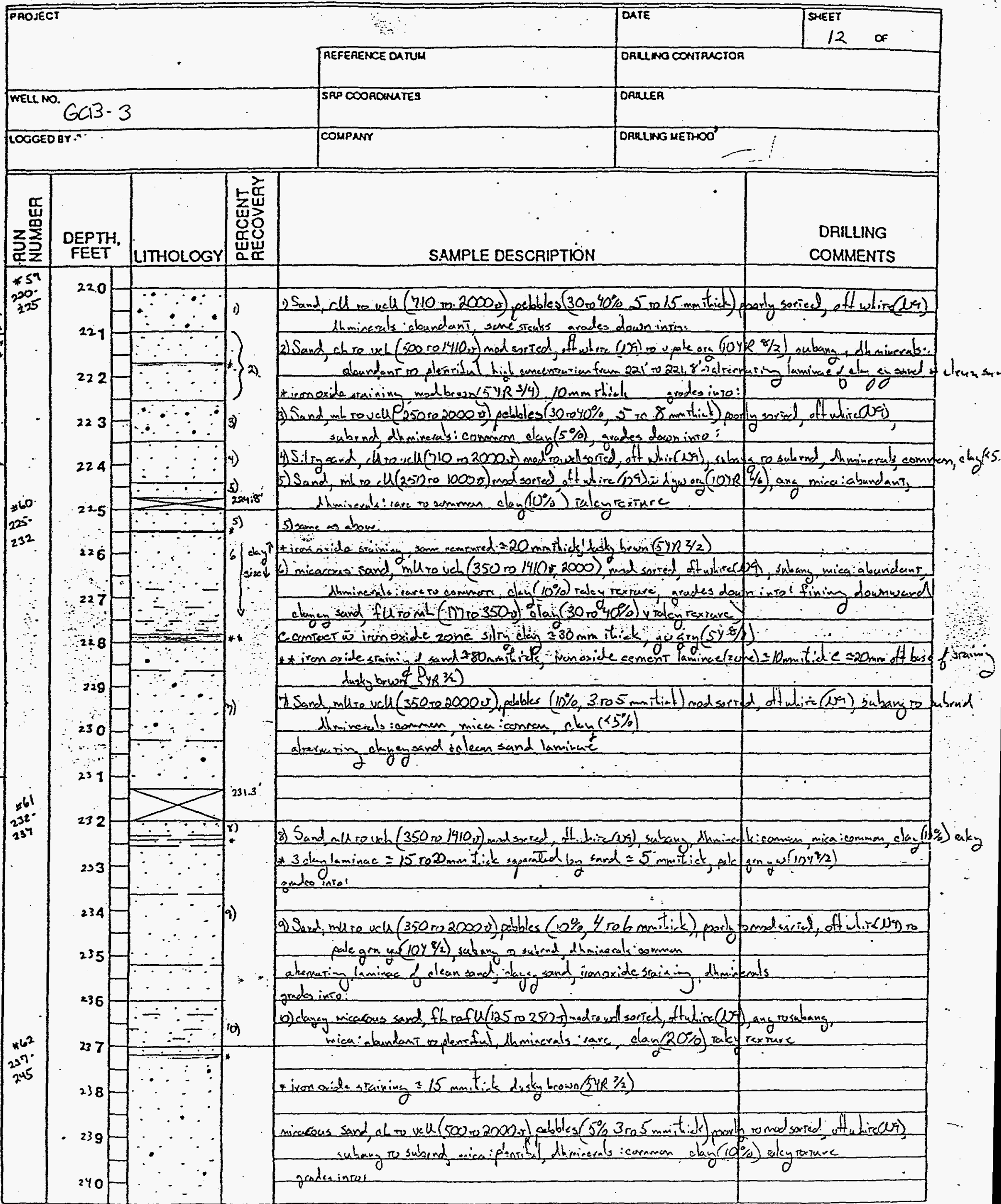


FIELD GEOLOGIC LOG

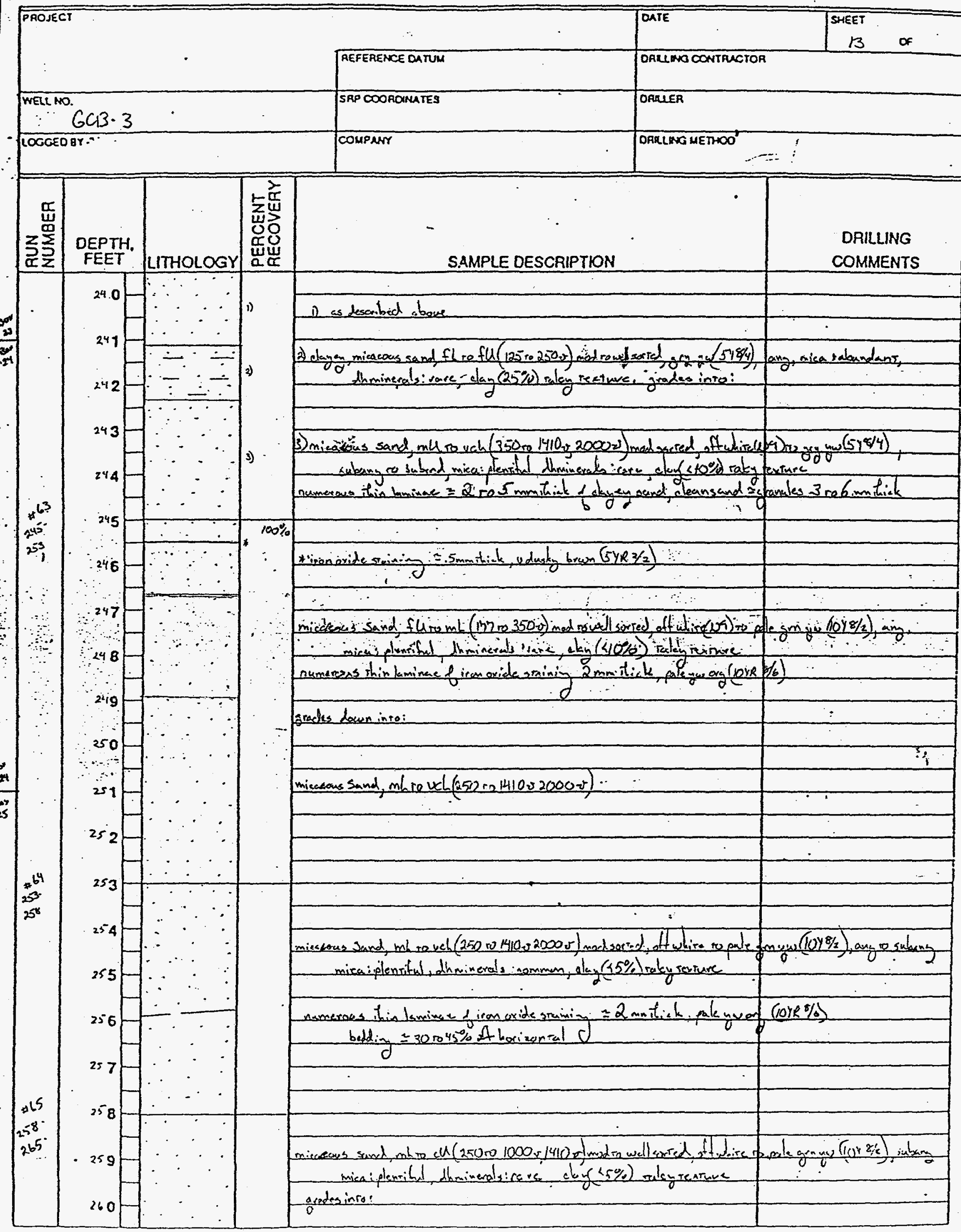




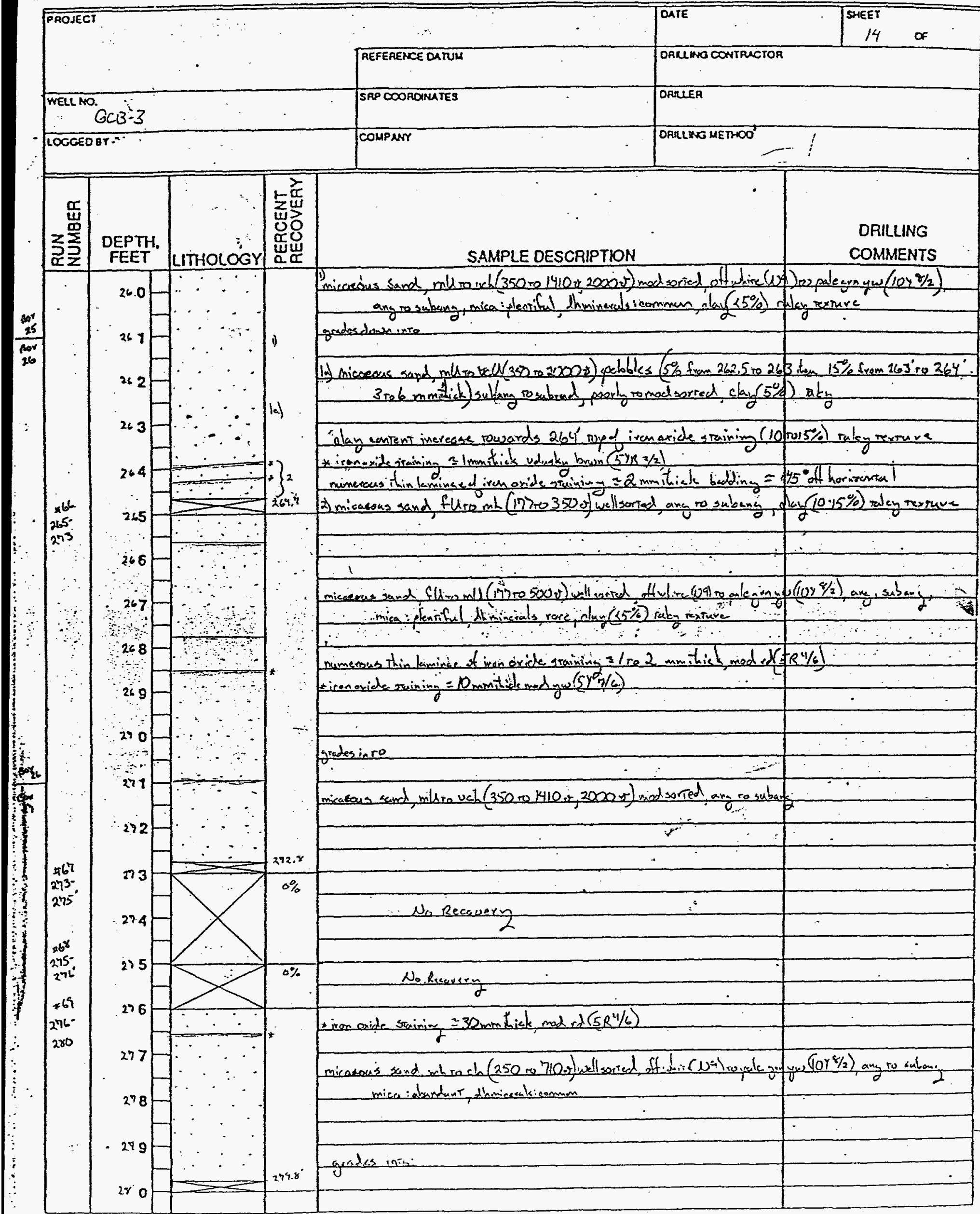




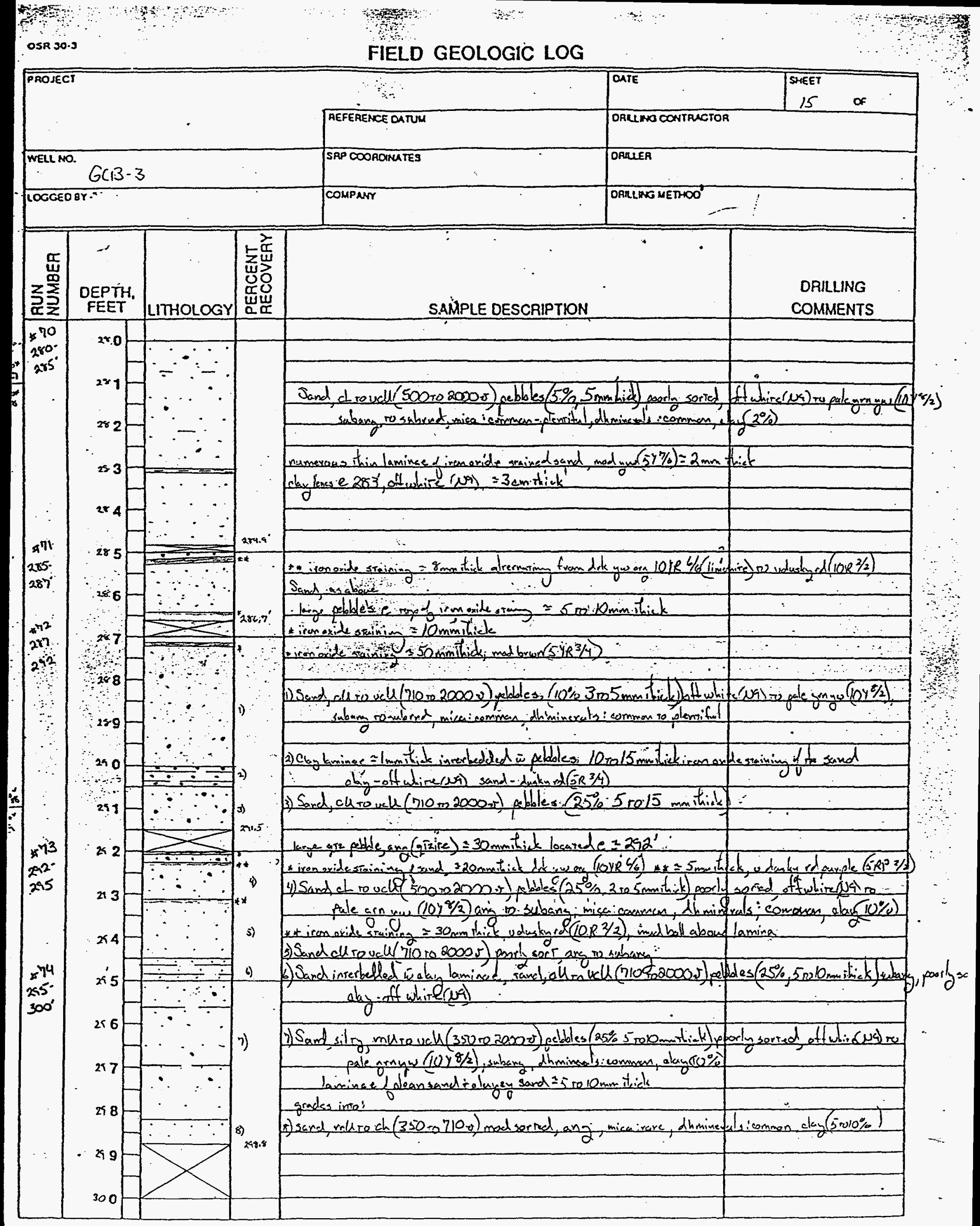


FIELD GEOLOGIC LOG

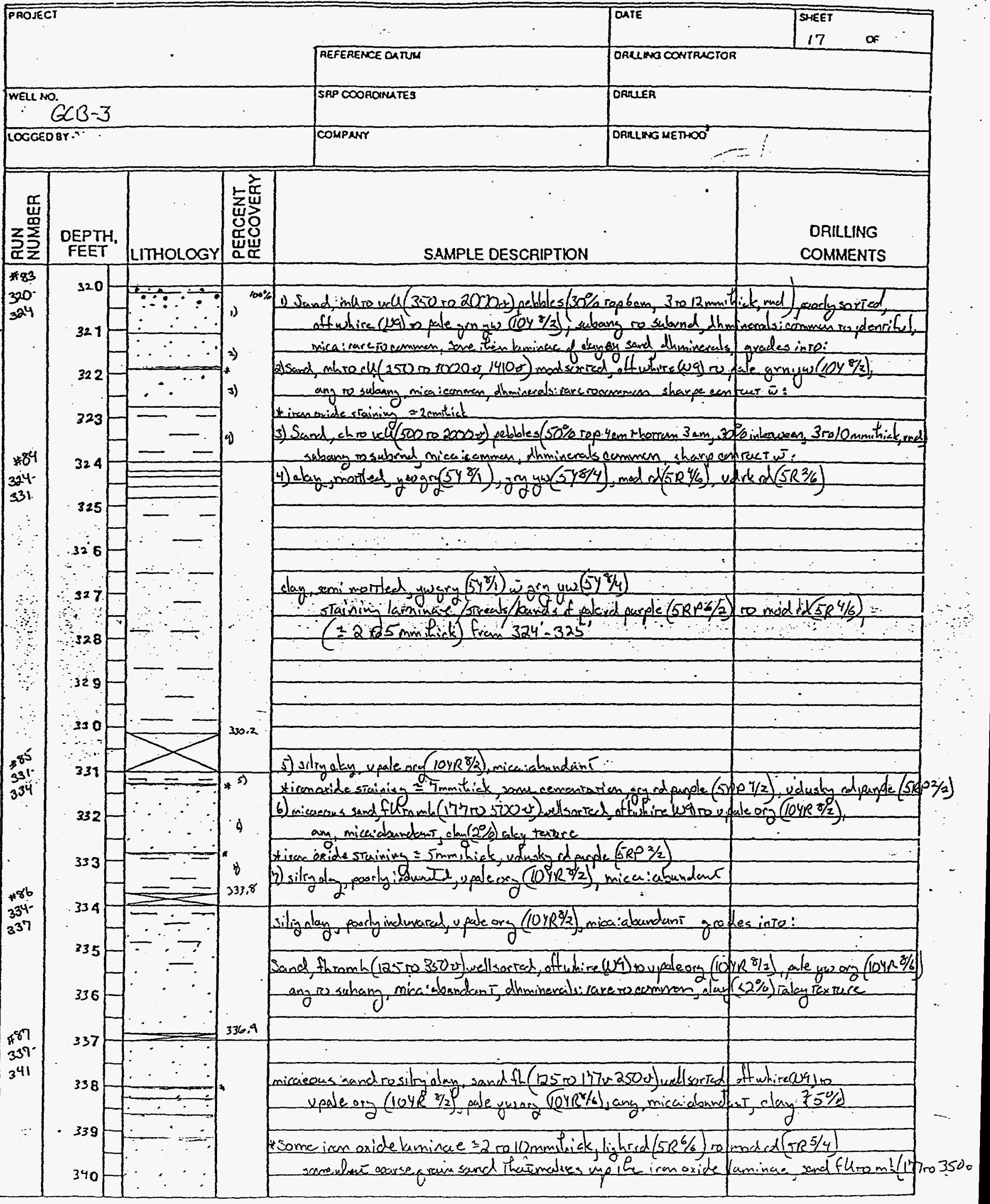




\section{3,3}

OSA 30.3

\section{FIELD GEOLOGIC LOG}

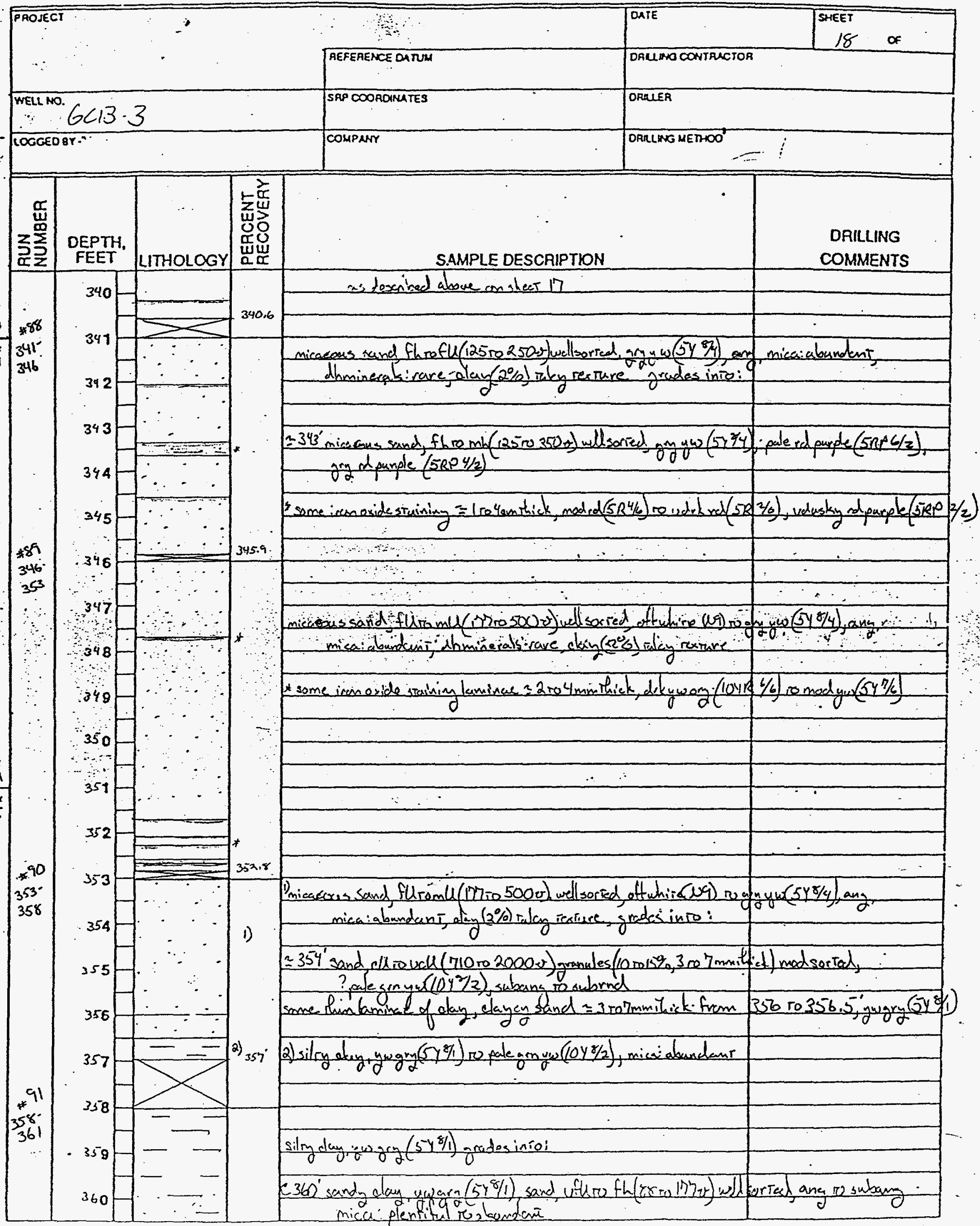


FIELD GEOLOGIC LOG

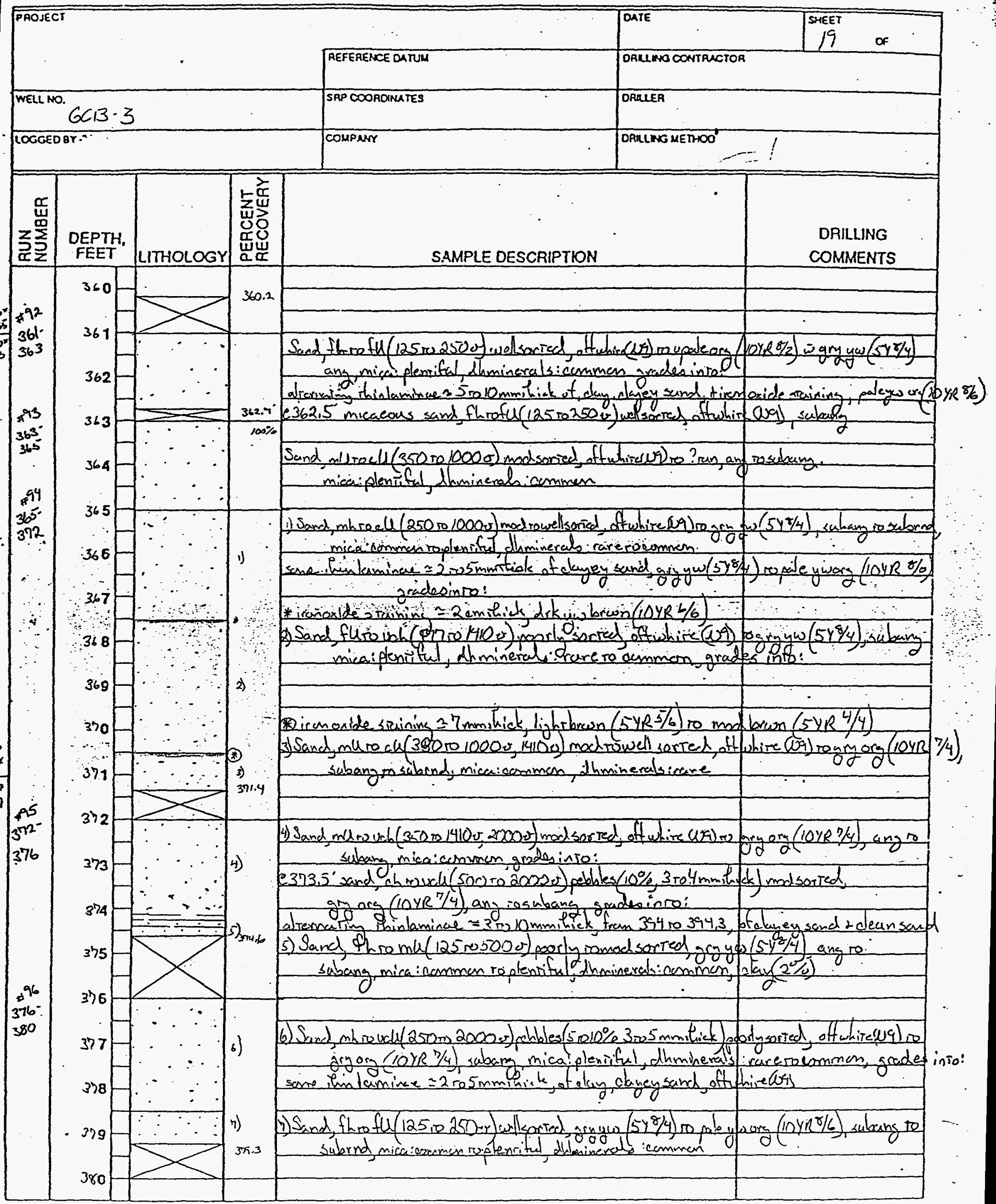




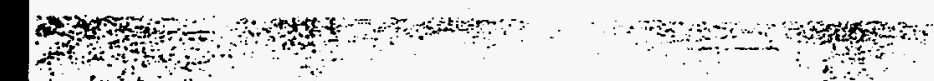

FIELD GEOLOGIC LOG

OSR 20.J

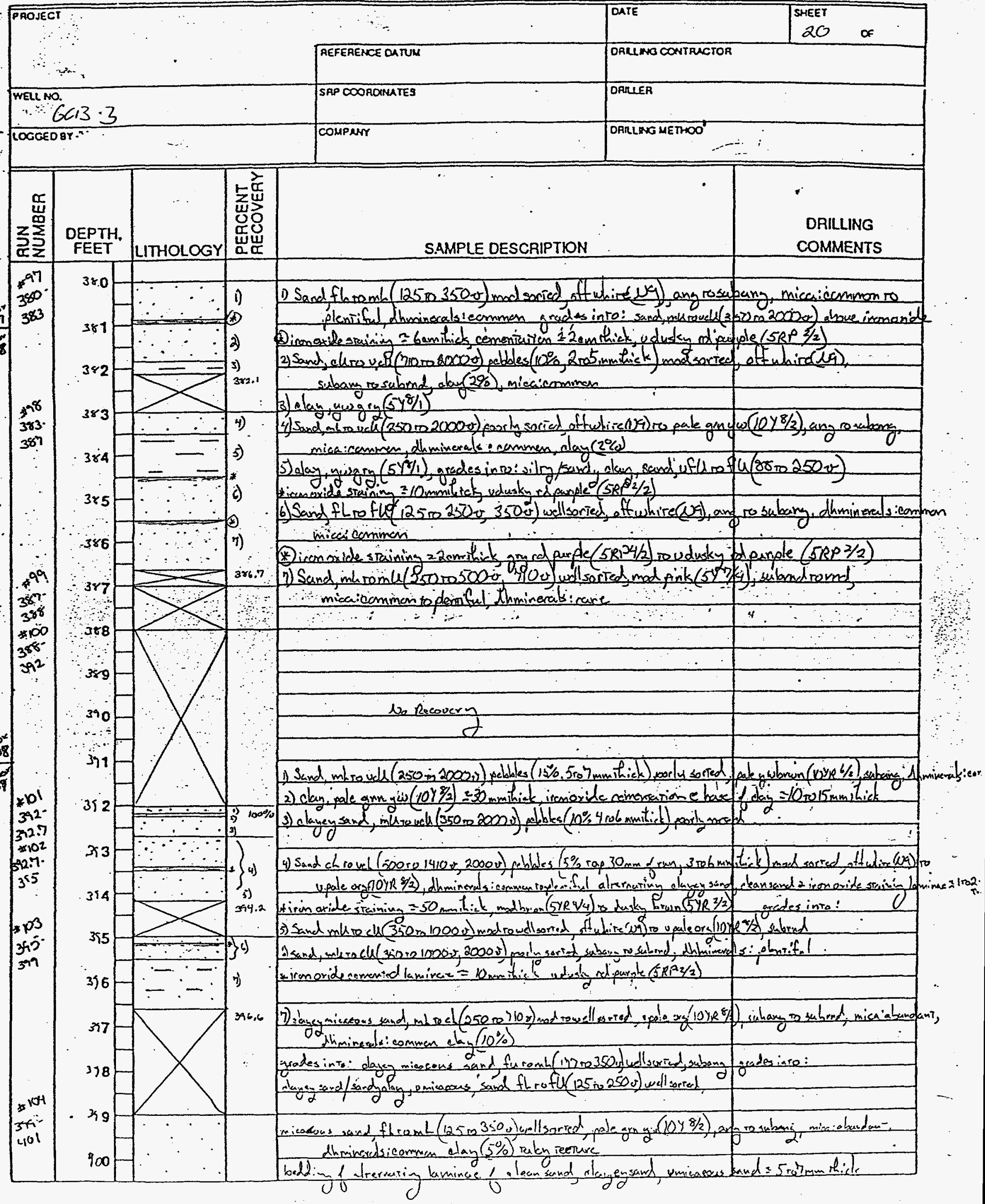




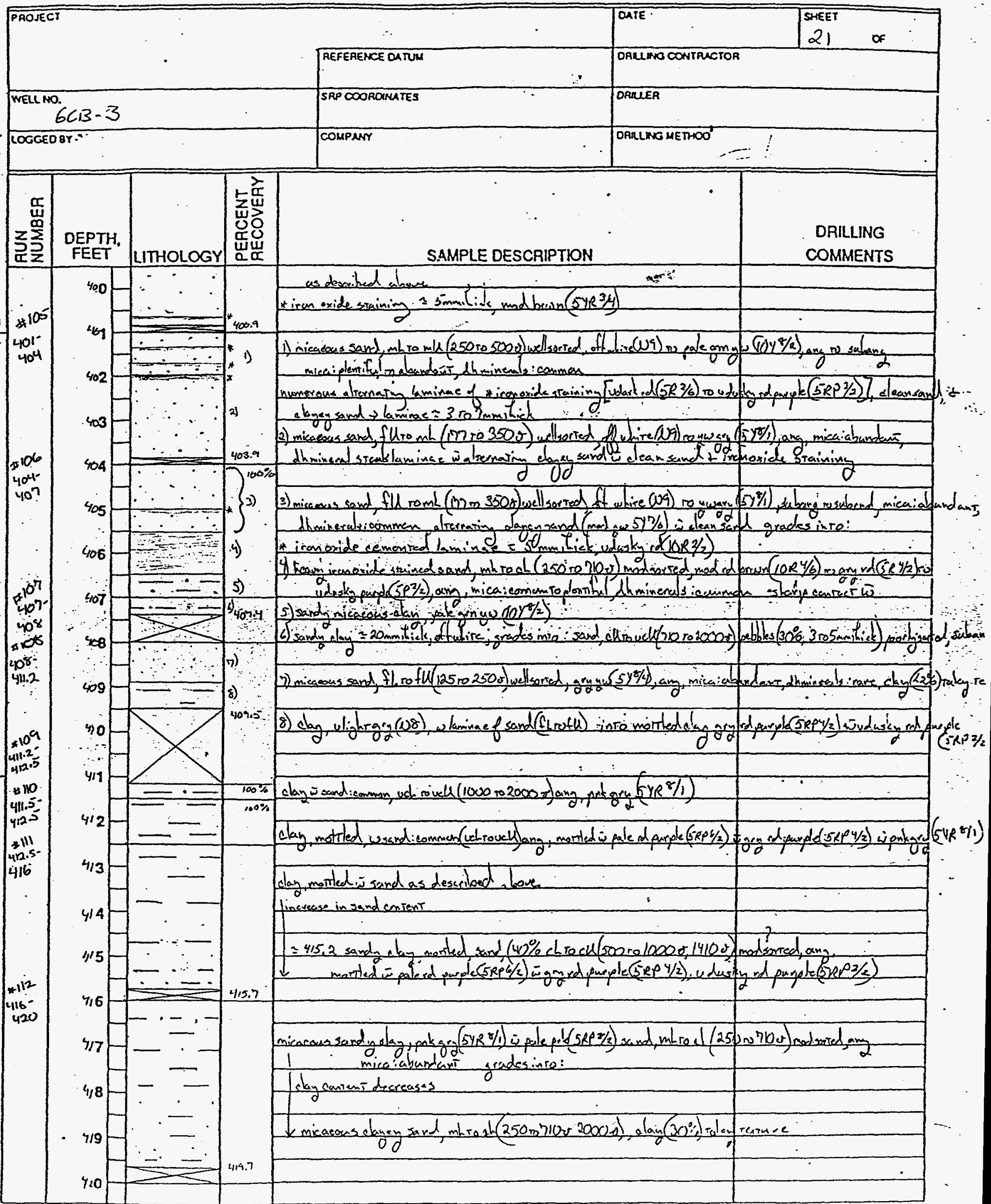




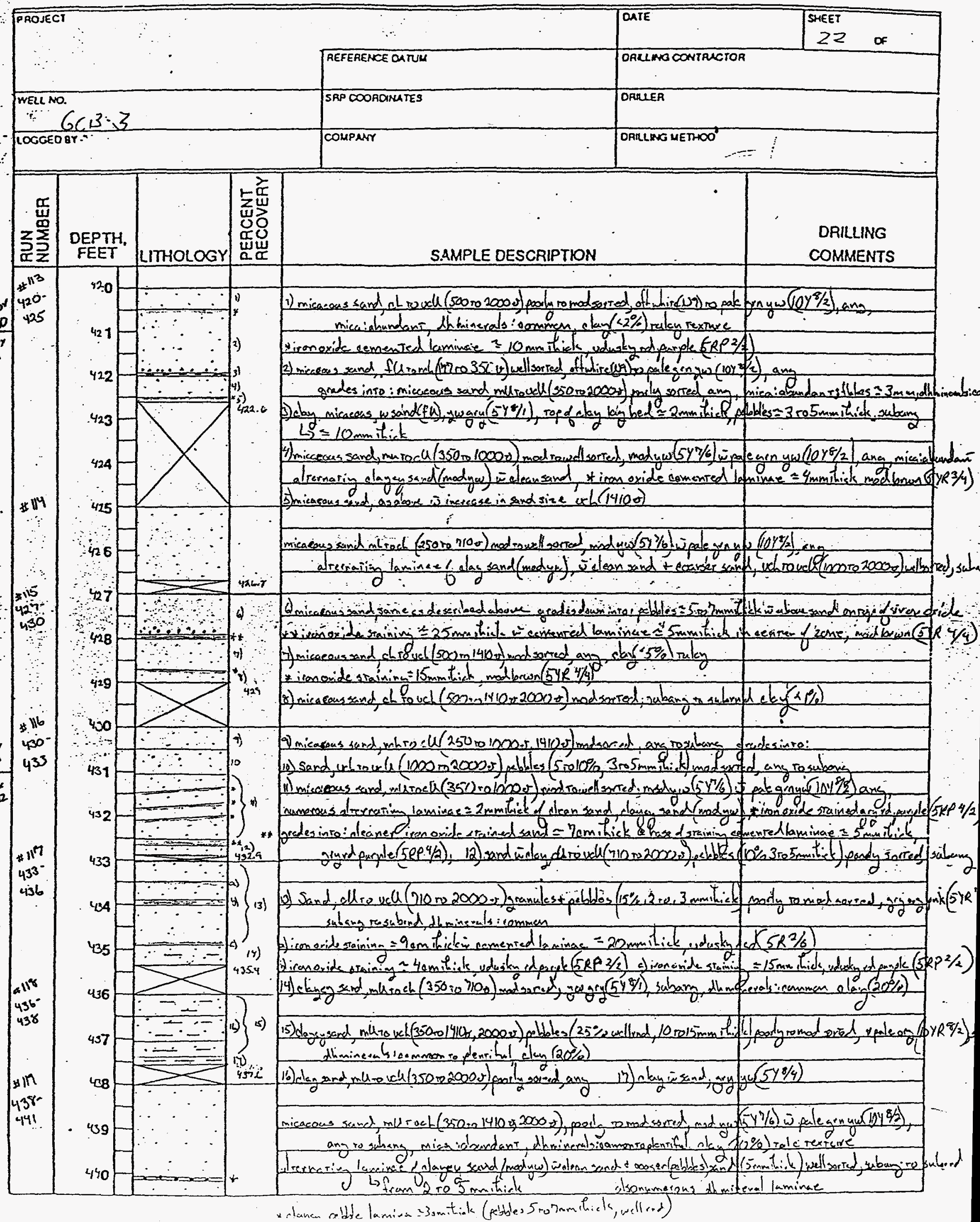




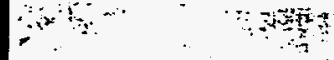

OSR 30.3

FIELD GEOLOGIC LOG

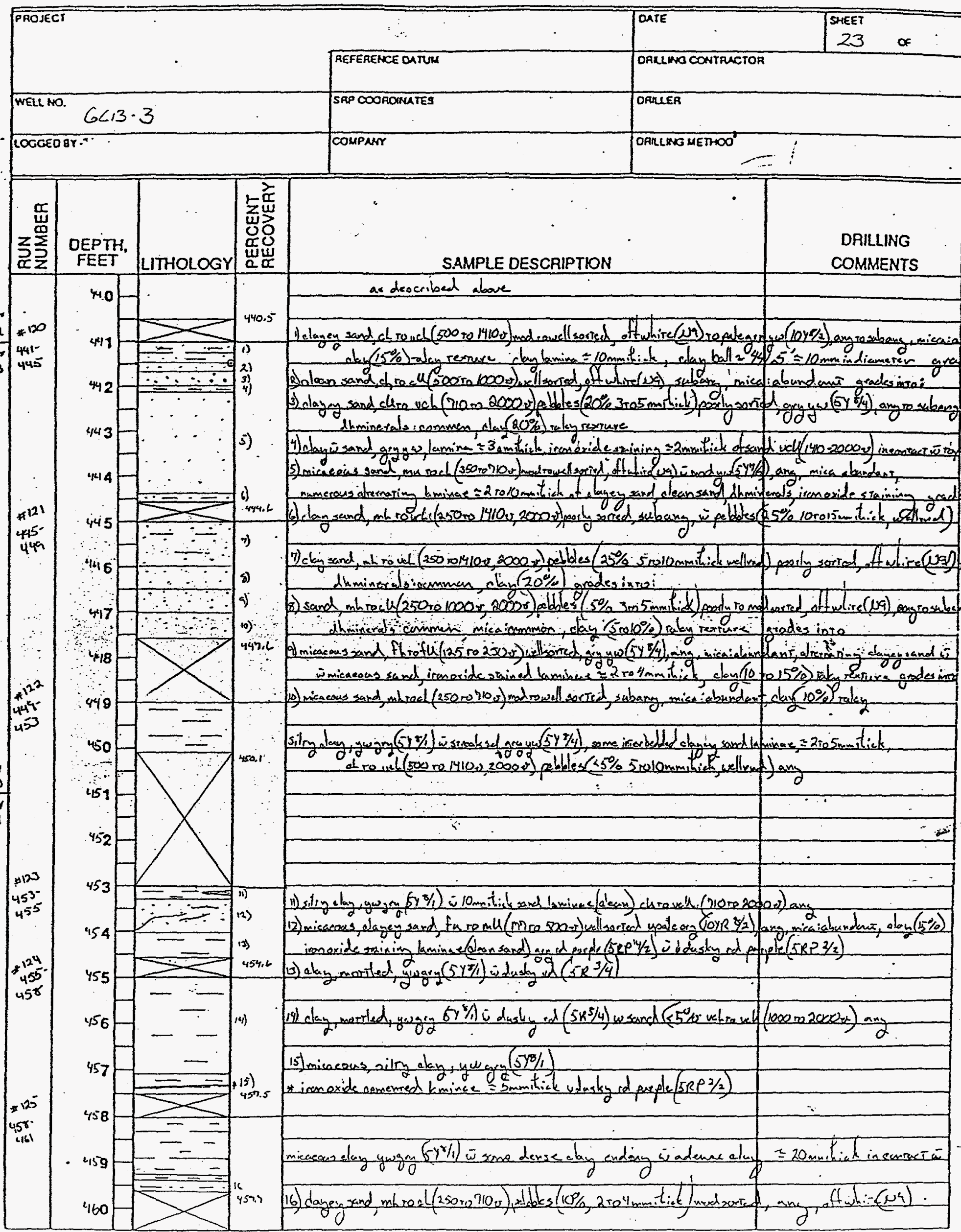




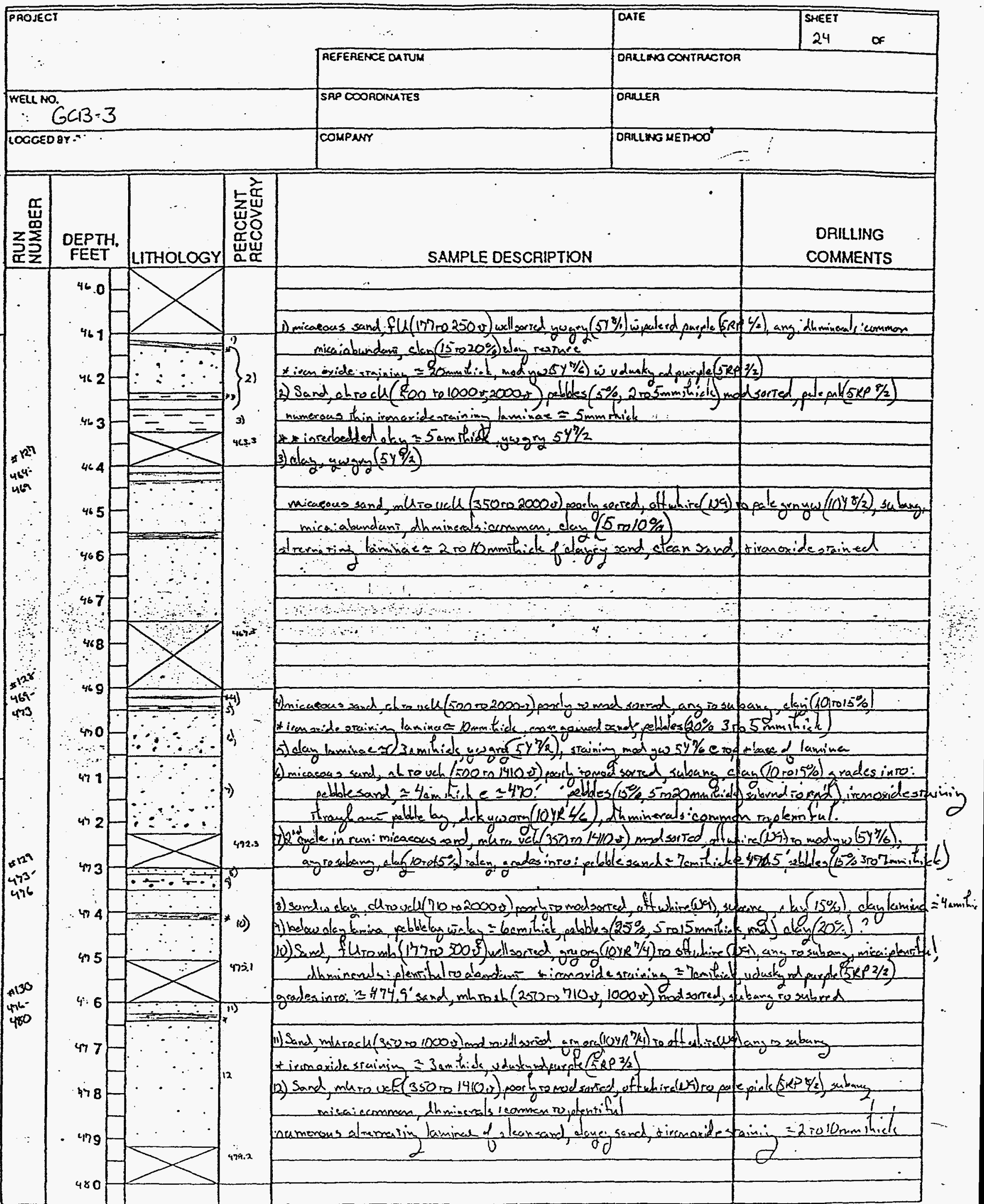




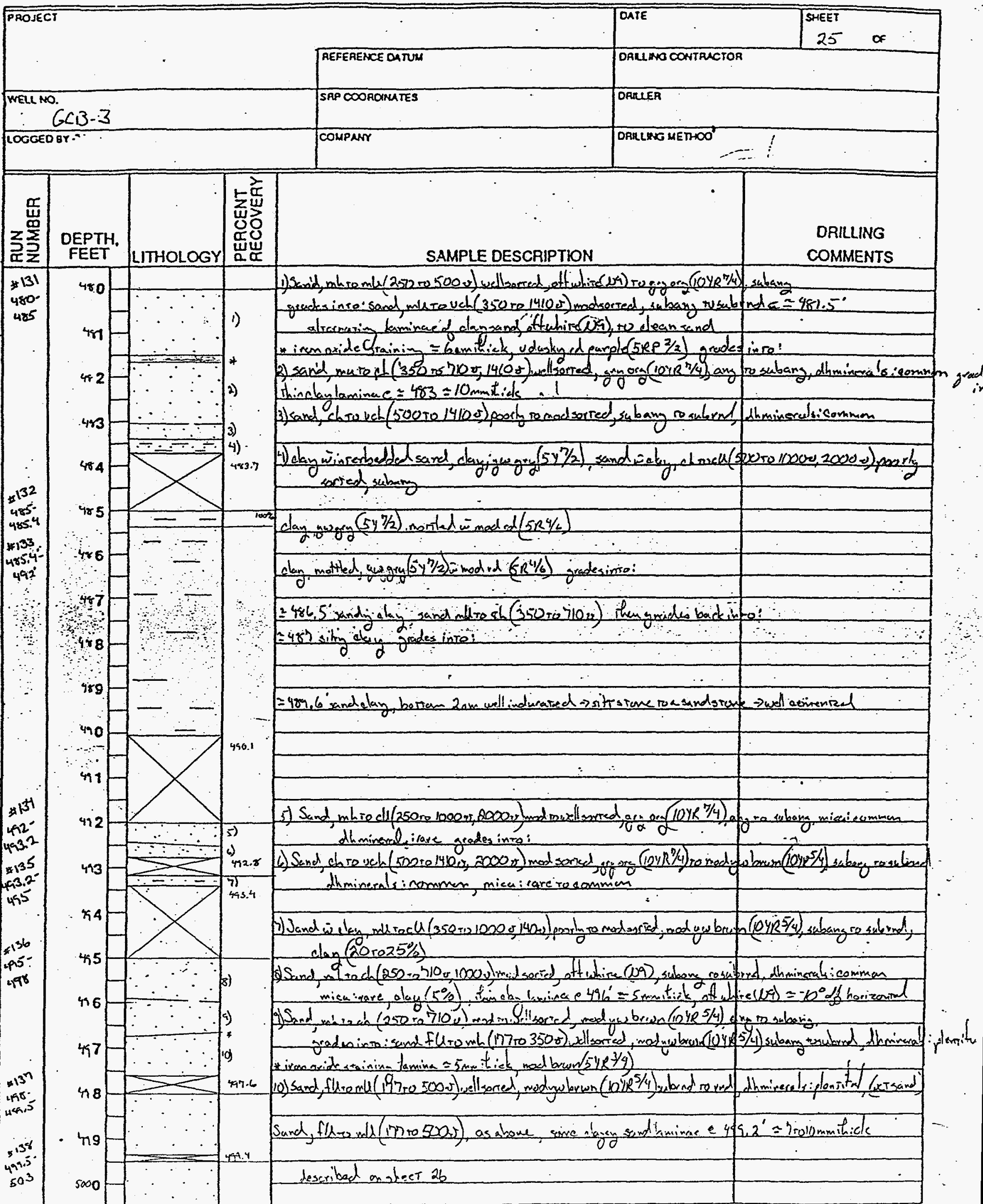




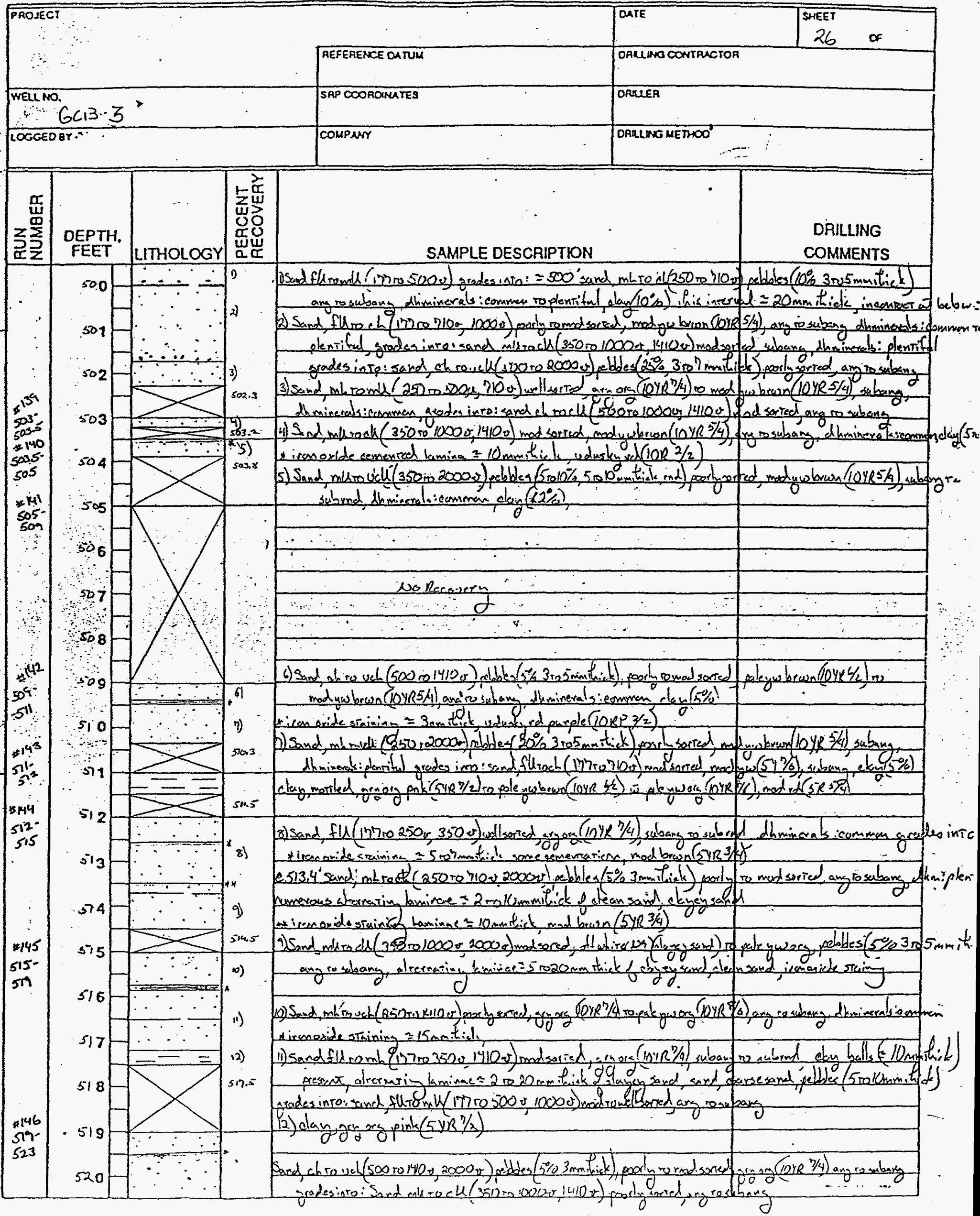




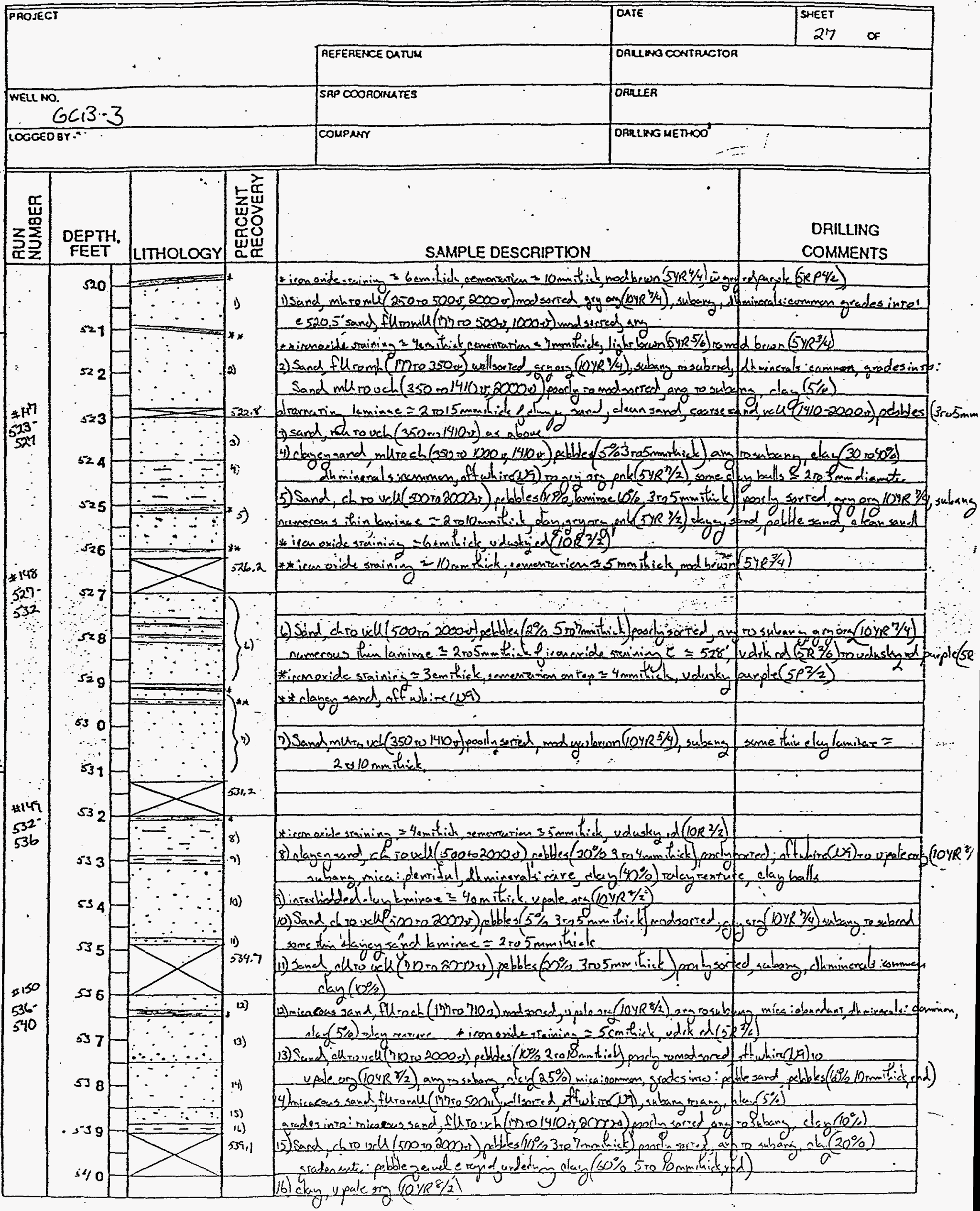




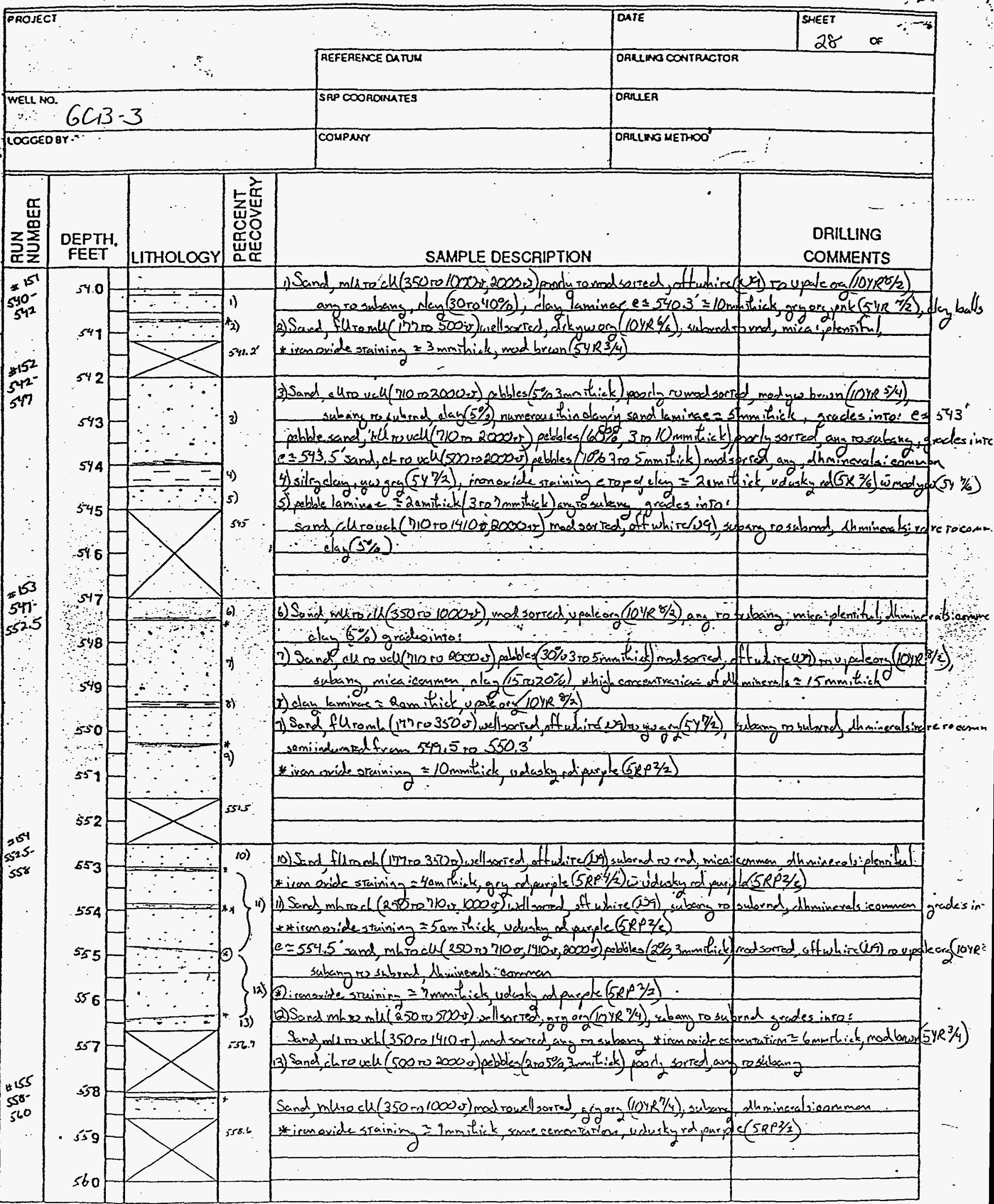


FIELD GEOLOGIC LOG

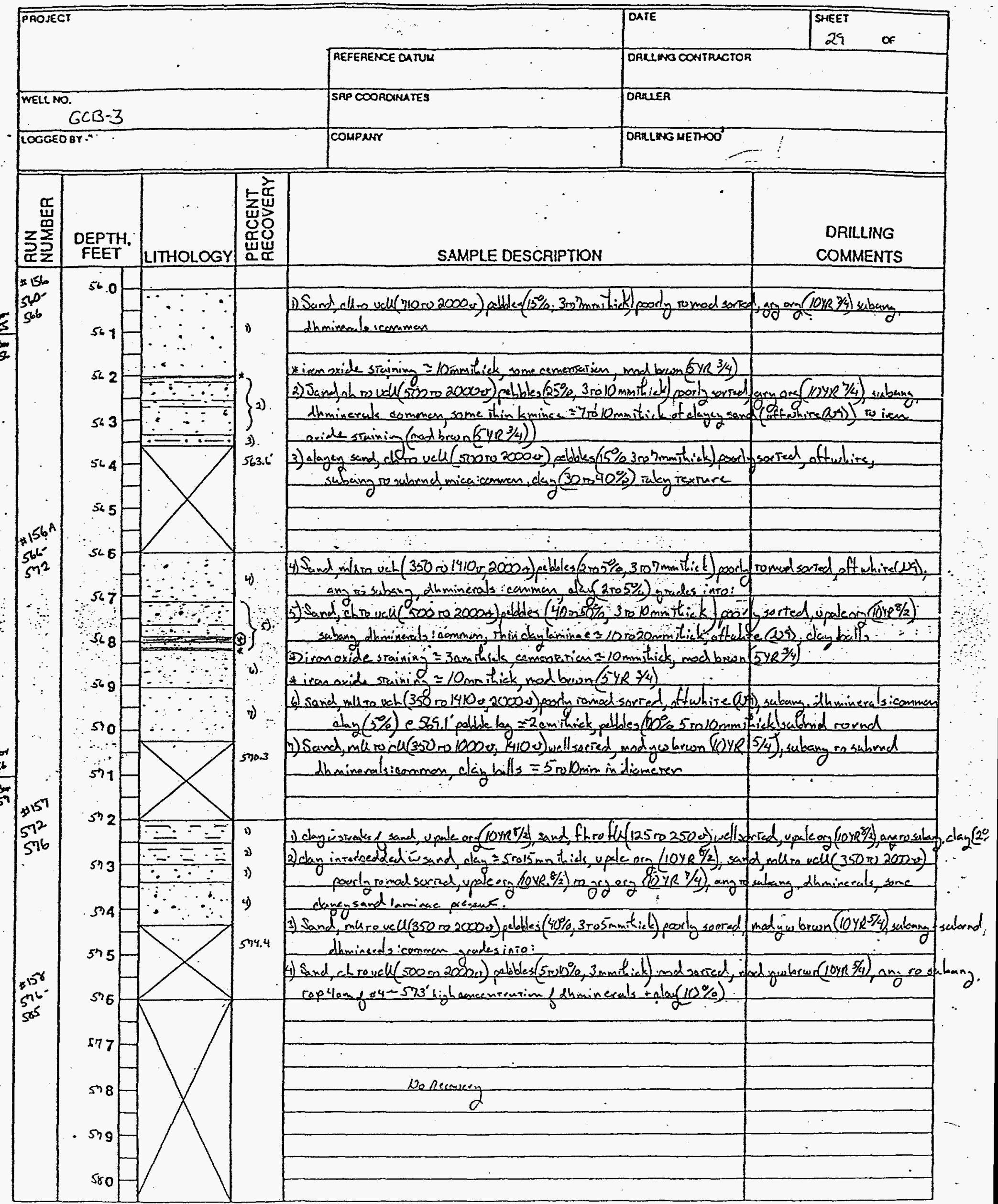




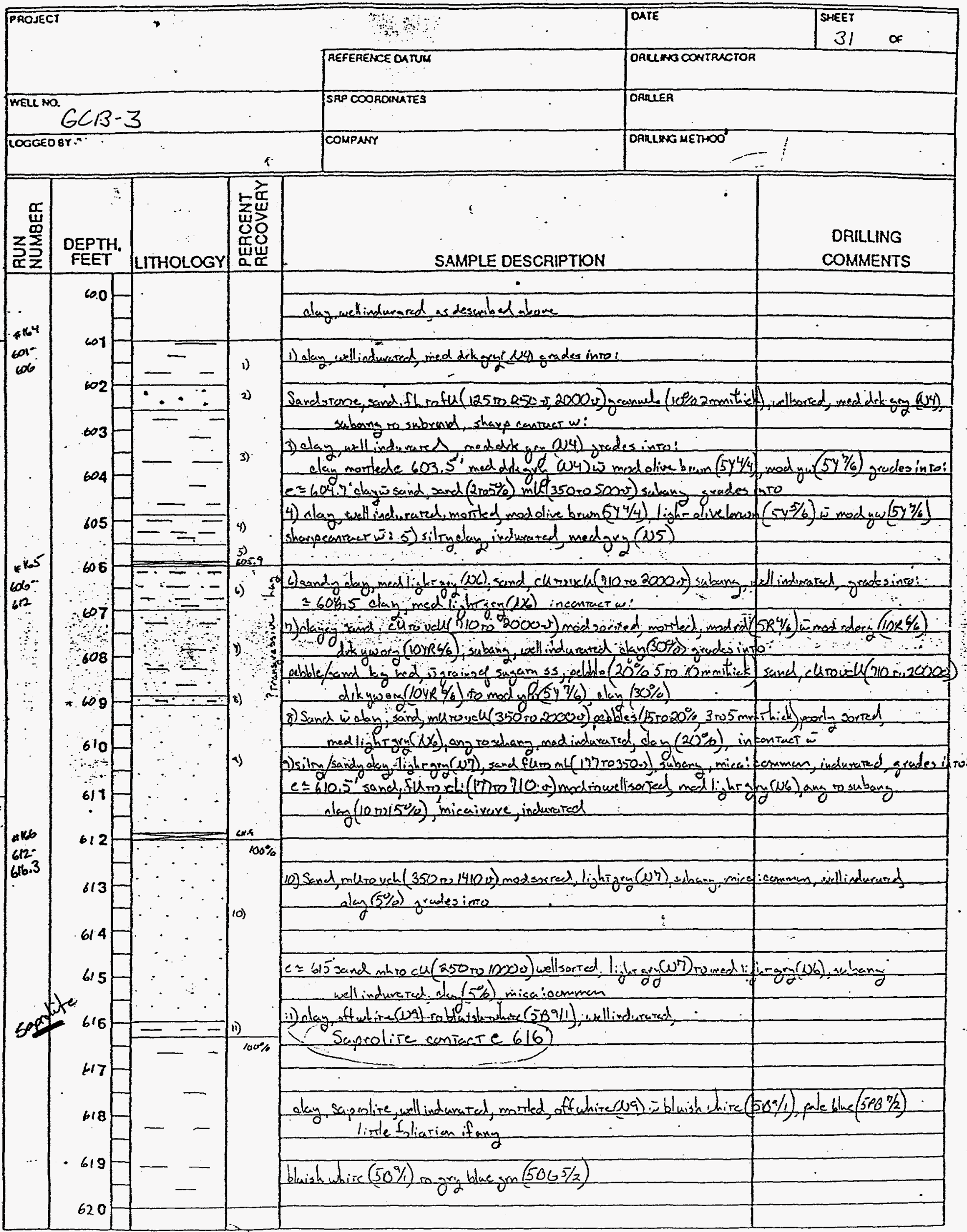


WSRC-RP-97-0185

Revision 0

June, 1997

\section{Appendix B}

Suspension Velocity Measurements for GCB-1 and 2

Note: GCB-4 and 5, ihcluded in this data, are not part of the AMM Study 
WSRC-RP-97-0185

Revision 0

June, 1997

This page intentionally left blank. 


\title{
SUSPENSION VELOCITY MEASUREMENTS
}

\author{
AT THE \\ SAVANNAH RIVER SITE \\ BOREHOLES GCB-1, GCB-4, AND GCB-5
}

\author{
Prepared for \\ University of South Carolina \\ Earth Sciences and Resources Institute \\ Columbia, South Carolina 29208 \\ (803) 331-6003 \\ Prepared by \\ Agbabian Associates, Inc. \\ 1111 South Arroyo Parkway, Suite 470 \\ Pasadena, California 91105 \\ (818) 441-1060 \\ Project 96227
}

October 31, 1996

Report 96227-6620 
INTRODUCTION

SCOPE OF WORK

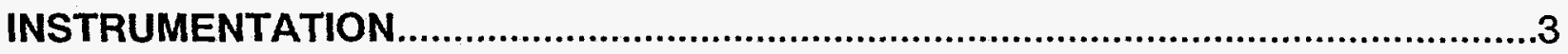

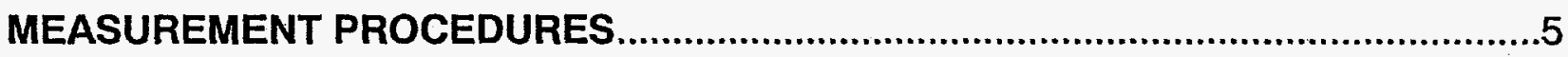

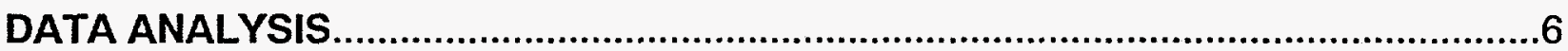

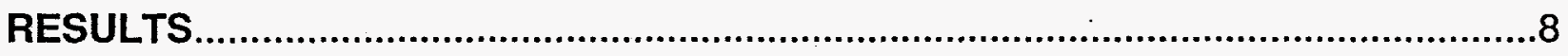

\section{SUMMARY}

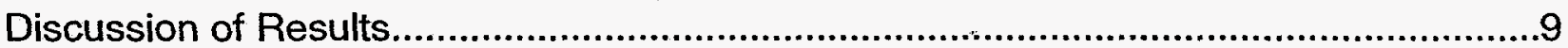

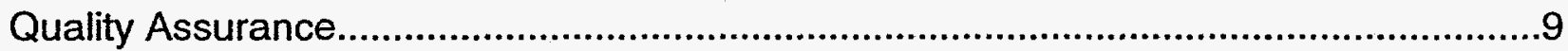

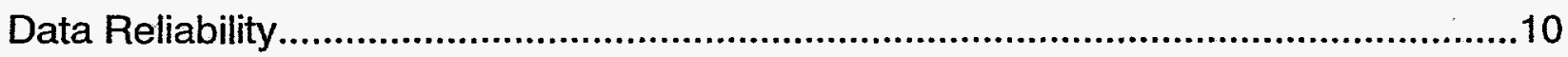




\section{FIGURES}

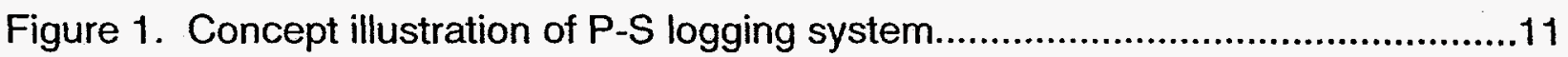

Figure 2. Filtered (2000 Hz lowpass) $567.6 \mathrm{ft}$ record from GCB-1 ...........................12

Figure 3. Unfiltered $567.6 \mathrm{ft}$ record from GCB-1 ..................................................13

Figure 4. GCB-1 Suspension R1 - R2 P-and $S_{H}$-wave velocities............................14

Figure 5. GCB-1 Suspension R1 - R2 $\mathrm{S}_{\mathrm{H}}$-wave velocities.....................................15

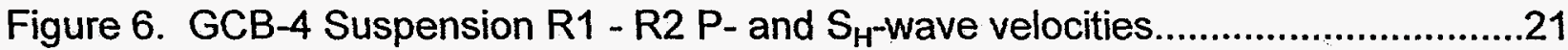

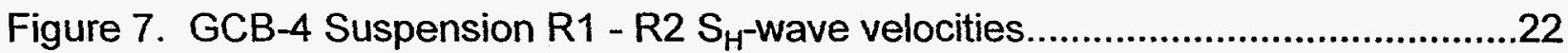

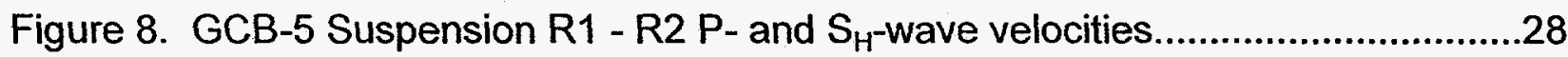

Figure 9. GCB-5 Suspension R1 - R2 $\mathrm{S}_{\mathrm{H}}$-wave velocities.......................................29

\section{TABLES}

Table 1. GCB-1 Suspension R1-R2 depth, pick times, and velocities.........................16

Table 2. GCB-4 Suspension R1-R2 depth, pick times, and velocities......................23

Table 3. GCB-5 Suspension R1-R2 depth, pick times, and velocities..........................30 


\section{APPENDICES}

APPENDIX A: Suspension velocity measurement quality assurance suspension source to receiver analysis results

APPENDIX B: Suspension S $_{H}$-wave R1 waveform records with S-R1 first arrival picks

APPENDIX C: Suspension P-wave R1 waveform records with S-R1 first arrival picks

APPENDIX D: OYO Model 170 suspension velocity logging system NIST traceable calibration procedure 


\section{INTRODUCTION}

Suspension velocity measurements were performed in boreholes GCB-1, GCB-4, and GCB-5 at the Savannah River Site, near Aiken, South Carolina. Suspension P- and $\mathrm{S}_{\mathrm{H}^{-}}$-velocity measurements were collected to a maximum depth of $1036.8 \mathrm{ft}$. Data acquisition was performed on September 4 and 29, 1996. The work was conducted by Rob Steller of Agbabian Associates for the University of South Carolina and the Westinghouse Savannah River Company. Mr. Mike Waddell was the technical liaison for the University of South Carolina, with Mr. Doug Wyatt serving as project liaison for Westinghouse.

This report describes the field measurements, data analysis; and results of this work. 


\section{SCOPE OF WORK}

This report presents the results of suspension velocity measurements on September 4 and 29, 1996, between depths of 4.9 and $1036.8 \mathrm{ft}$ in the nominal 6 inch uncased boreholes designated GCB-1, GCB-4, and GCB-5, located at the Savannah River Site, near Aiken, South Carolina. The purpose of these measurements was to acquire shear wave velocities and compressional wave velocities as a function of depth which, in turn, will be used to characterize ground response to earthquake motion.

The OYO Model 170 Suspension Logging Recorder and Suspension Logging Probe were used to obtain in-situ horizontal shear and compressional wave velocity measurements at 3.28 intervals. The acquired data was analyzed and a profile of velocity versus depth was produced for both compressional and horizontally polarized shear waves.

A detailed reference for the velocity measurement techniques used in this study is:

Guidelines for Determining Design Basis Ground Motions, Report TR-102293, Electric Power Research Institute, Palo Alto, California, November 1993, Sections 7 and 8. 


\section{INSTRUMENTATION}

Suspension soil velocity measurements were performed using the Model 170 Suspension Logging system, manufactured by OYO Corporation. This system directly determines the average velocity of a $3.28 \mathrm{ft}$ high segment of the soil column surrounding the borehole of interest by measuring the elapsed time between arrivals of a wave propagating upward through the soil column. The geophones that detect the wave, and the source that generates the wave, are moved as a unit in the borehole producing relatively constant amplitude signals at all depths.

The suspension system probe consists of a combined reversible polarity solenoid horizontal shear-wave generator $\left(\mathrm{S}_{\mathrm{H}}\right)$ and compressional-wave generator $(\mathrm{P})$, joined to two biaxial geophones by a flexible isolation cylinder, as shown in Figure 1. The separation of the two geophones is $3.28 \mathrm{ft}$, allowing average wave velocity in the region between the geophones to be determined by inversion of the wave travel time between the two geophones. The total length of the probe as used in this survey is approximately $19.0 \mathrm{ft}$; the center point of the geophones is approximately $12.1 \mathrm{ft}$ above the bottom end of the probe. The probe receives control signals from, and sends the amplified geophone signals to, instrumentation on the surface via an armored 7 conductor cable. The cable is wound onto the drum of a winch and is used to support the probe. Cable travel is measured to provide probe depth data.

The entire probe is suspended by the cable and centered in the borehole by nylon "whiskers", therefore, source motion is not coupled directly to the borehole walls; rather, the source motion creates a horizontally propagating impulsive pressure wave in the fluid filling the borehole and surrounding the source. This pressure wave is converted to $\mathrm{P}$ and $\mathrm{S}_{\mathrm{H}}$-waves in the surrounding soil and rock as it impinges upon the borehole wall. These waves propagate through the soil and rock surrounding the borehole, in turn causing a pressure wave to be generated in the fluid surrounding the géophones as the soil waves pass their location.

In operation, a distinct, repeatable pattern of impulses is generated at each depth as follows:

1. The source is fired in one direction producing dominantly horizontal shear with some vertical compression, and the signals from the horizontal geophones situated parallel to the axis of motion of the source are recorded. 
2. The source is fired again in the opposite direction and the horizontal geophone signals are recorded.

3. The source is fired again and the vertical geophone signals are recorded. The repeated source pattern facilitates the picking of the $\mathrm{P}$ and $\mathrm{S}_{\mathrm{H}^{-}}$wave arrivals; reversal of the source changes the polarity of the $\mathrm{S}_{\mathrm{H}^{-}}$-wave pattern but not the $\mathrm{P}$-wave pattern.

The data from each geophone during each source activation is recorded as a different channel on the recording system. The Model 170 has six channels (two simultaneous recording channels), each with a 12 bit 1024 sample record. The recorded data is displayed on a CRT display and on paper tape output as six channels with a common time scale. Data is stored on 3.5 inch floppy diskettes for further processing. Up to 8 sampling sequences can be summed to improve the signal to noise ratio of the signals.

Review of the displayed data on the CRT or paper tape allows the operator to set the gains, filters, delay time, pulse length (energy), sample rate, and summing number in order to optimize the quality of the data before recording. Calibration of the Model 170 digital recorder is performed every six months, using a NITS traceable frequency source and counter, as outlined in Appendix D. 
All boreholes were logged as nominal 6 in diameter uncased boreholes filled with bentonite based drilling fluid. The suspension probe was positioned with the mid-point of the receiver spacing at grade level, and mechanical and electronic depth counters were set to zero. The probe was lowered to the bottom of the borehole, then returned to the bottom of the steel conductor casing or mud level, stopping at $3.28 \mathrm{ft}$ intervals to collect data.

\begin{tabular}{|c|c|c|c|c|c|c|}
\hline BOREHOLE & $\begin{array}{l}\text { RUN } \\
\text { NUMBER }\end{array}$ & $\begin{array}{l}\text { DEPTH } \\
\text { RANGE }\end{array}$ & $\begin{array}{l}\text { DEPTH AS } \\
\text { DRILLED }\end{array}$ & $\begin{array}{ll}\text { LOST } & \text { TO } \\
\text { SLUFF } & \end{array}$ & $\begin{array}{l}\text { SAMPLE } \\
\text { INTERVAL }\end{array}$ & $\begin{array}{l}\text { ISOLATION } \\
\text { TUBE }\end{array}$ \\
\hline \multirow{2}{*}{$\begin{array}{l}\text { GCB-1 } \\
9 / 4 / 96\end{array}$} & 1 & $789.0-111.6$ & 802 & 0.9 & 3.28 & 3.28 \\
\hline & & & & & & \\
\hline \multirow{2}{*}{$\begin{array}{l}\text { GCB-4 } \\
9 / 29 / 96\end{array}$} & 1 & $1023.6-787.4$ & & & 3.28 & 3.28 \\
\hline & 2 & $\begin{array}{l}1036.8-1010.5 \\
784.1-13.1\end{array}$ & 1100 & 51.1 & 3.28 & 3.28 \\
\hline \multirow{2}{*}{$\begin{array}{l}\text { GCB-5 } \\
9 / 29 / 96\end{array}$} & 1 & $4.9-981.0$ & 995 & 1.9 & 3.28 & 3.28 \\
\hline & & & & & & \\
\hline
\end{tabular}

At each measurement depth the measurement sequence of two opposite horizontal records and one vertical record was performed, and the gains were adjusted as required. The data from each depth was printed on paper tape, checked, and recorded on diskette before moving to the next depth.

Upon completion of the measurement run, the probe zero depth indication was verified prior to removal from the borehole. 


\section{DATA ANALYSIS}

The recorded digital records were analyzed to locate the first minima on the vertical axis records, indicating the arrival of P-wave energy. In the case of GCB-4 and GCB-5, The vertical axis records showed no arrivals, due to equipment malfunction. This problem was identified in the field and not corrected due to time constraints. The P-wave arrivals for these boreholes were picked from redundant data included in the unfiltered horizontal axis records, and transfered to the vertical axis records. The difference in travel time between receiver 1 and receiver 2 (R1-R2) arrivals was used to calculate the $\mathrm{P}$-wave velocity for that $3.28 \mathrm{ft}$ interval. When observable, $\mathrm{P}$ wave arrivals on the horizontal axis records were used to verify the velocities determined from the vertical axis data.

The P-wave velocity calculated from the travel time over the $7.02 \mathrm{ft}$ interval from source to receiver 1 (S-R1) was calculated and plotted for quality assurance of the velocity derived from the travel time between receivers. In this analysis, the depth values as recorded were increased by $5.15 \mathrm{ft}$ to correspond to the mid-point of the $7.02 \mathrm{ft} \mathrm{S-R} 1$ interval. Travel times were obtained by picking the first break of the P-wave signal at the near receiver and subtracting 3.90 milliseconds, the calculated and experimentally verified delay from source trigger pulse (beginning of record) to source impact. This delay corresponds to the duration of acceleration of the solenoid before impact.

The recorded digital records were studied to establish the presence of clear $\mathrm{S}_{\mathrm{H}}$-wave pulses, as indicated by the presence of opposite polarity pulses on each pair of horizontal records. Ideally, the $\mathrm{S}_{\mathrm{H}}$-wave signals from the 'normal' and 'reverse' source pulses are very nearly inverted images of each other. Digital FFT - IFFT lowpass filtering was used to remove the higher frequency Pwave signal from the $\mathrm{S}_{\mathrm{H}^{-}}$wave signal. Different filter cutoffs were used to separate $\mathrm{P}$ - and $\mathrm{S}_{\mathrm{H}^{-}}$ waves at different depths, ranging from $1000 \mathrm{~Hz}$ in the slowest zones to $4000 \mathrm{~Hz}$ in the regions of highest velocity.

Generally, the first maxima was picked for the 'normal' signals and the first minima for the 'reverse' signals, although other points on the waveform were used if the first pulse was distorted. The absolute arrival time of the 'normal' and 'reverse' signals may vary by $+/-0.2$ milliseconds, 
due to differences in the actuation time of the solenoid source caused by constant mechanical bias in the source or by borehole inclination. This variation does not affect the R1-R2 velocity determinations, as the differential time is measured between arrivals of waves created by the same source actuation. The final velocity value is the average of the values obtained from the 'normal' and 'reverse' source actuations.

As with the $\mathrm{P}$-wave data, $\mathrm{S}_{\mathrm{H}^{-}}$-wave velocity calculated from the travel time over the $7.02 \mathrm{ft}$ interval from source to first receiver was calculated and plotted for verification of the velocity derived from the travel time between receivers. In this analysis, the depth values were increased by $5.15 \mathrm{ft}$ to correspond to the mid-point of the $7.02 \mathrm{ft} \mathrm{S-R} 1$ interval. Travel times were obtained by picking the first break of the $\mathrm{S}_{\mathrm{H}}$-wave signal at the near receiver and subtracting 3.90 milliseconds, the calculated and experimentally verified delay from the beginning of the record at the source trigger pulse to source impact.

Figure 2 shows an example of R1 - R2 measurements on the filtered record for a depth of 567.6 $\mathrm{ft}$ in borehole GCB-1. In Figure 2, the time difference over the $3.28 \mathrm{ft}$ interval of 1.30 millisecond is equivalent to a $\mathrm{S}_{\mathrm{H}}$-wave velocity of $2524 \mathrm{ft} / \mathrm{sec}$. Whenever possible, time differences were determined from several phase points on the $\mathrm{S}_{\mathrm{H}^{-}}$-waveform records to verify the

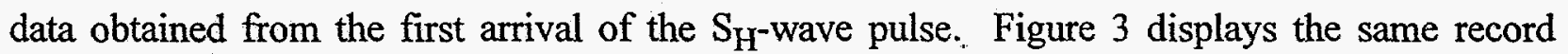
before filtering with a $2000 \mathrm{~Hz}$ FFT - IFFT digital lowpass filter. 


\section{RESULTS}

Suspension R1-R2 P- and $\mathrm{S}_{\mathrm{H}^{-}}$wave velocities for GCB1, GCB-4, and GCB-5 are plotted in Figures 4,6 , and 8 respectively. The $\mathrm{S}_{\mathrm{H}^{-}}$wave velocity data are presented at an expanded scale in Figures 5, 7, and 9. The suspension velocity data presented in the figures are presented in Tables $1-3$. $\mathrm{P}$ - and $\mathrm{S}_{\mathrm{H}}$-wave velocity data from $\mathrm{R} 1-\mathrm{R} 2$ analysis and quality assurance analysis of S-R1 data are plotted together in Appendix A, Figures A1 - A6 to aid in visual comparison. It must be noted that R1-R2 data is an average velocity over a $3.28 \mathrm{ft}$ segment of the soil column; $\mathrm{S}-\mathrm{R} 1$ data is an average over $7.02 \mathrm{ft}$, creating a significant smoothing relative to the R1-R2 plots. S-R1 data are presented in tabular format in Appendix A, Tables A1 - A3. Good correspondence between the shape of the $\mathrm{P}$ - and $\mathrm{S}_{\mathrm{H}^{-}}$wave velocity curves is observed for all data sets. The velocities derived from S-R1 and R1-R2 data are in close agreement, providing verification of the higher resolution R1-R2 data.

Depth sequential $\mathrm{S}_{\mathrm{H}^{-}}$and P-wave records with picks from S-R1 analysis used for quality assurance are presented in Appendices B and C. Calibration procedures and records for the measurement system are presented in Appendix D. 


\section{SUMMARY}

\section{Discussion of Results}

Both $\mathrm{P}$ - and $\mathrm{S}_{\mathrm{H}^{-}}$-wave velocities were measured using the Suspension Method between depths of 4.9 and $1036.8 \mathrm{ft}$ in boreholes GCB-1, GCB-4, and GCB-5 at the Savannah River Site. Data was not collected above $111.6 \mathrm{ft}$ in GCB-1 due to the rapid loss of drilling fluid above this point.

The sites were in remote locations, with no apparent sources of ambient vibration nearby.

Each of the sites indicates contact with basement like material, although GCB-4 does not appear to penetrate through the weathered region into competent rock.

\section{Quality Assurance}

These velocity measurements at the Savannah River Site, boreholes GCB-1, GCB-4, and GCB-5 were performed by highly qualified professional staff members of Agbabian Associates, Inc. under the supervision and quality assurance of Dr. Robert L. Nigbor, P.E.. Industry-standard or better methods and levels of care were employed for both measurements and analyses. All work was performed under Agbabian Associates quality assurance procedures, which include:

- Use of NIST-traceable calibrations, where applicable, for field and laboratory instrumentation

- Use of standard field data logs

- Where applicable, use of independent verification of data (for example, comparison of receiver-to-receiver and source-to-receiver velocities for the suspension velocity measurements)

- Independent review of calculations and results by a registered professional engineer, geologist, or geophysicist. 


\section{Data Reliability}

$\mathrm{P}$ - and $\mathrm{S}_{\mathrm{H}}$-wave velocity measurement using the Suspension Method gives average velocities over a $3.28 \mathrm{ft}$ interval of depth. This high resolution results in the scatter of values shown in the graphs. Individual measurements are very reliable; the estimated precision is $+/-5 \%$. Good field procedures and quality assurance add to the high reliability of these data. 


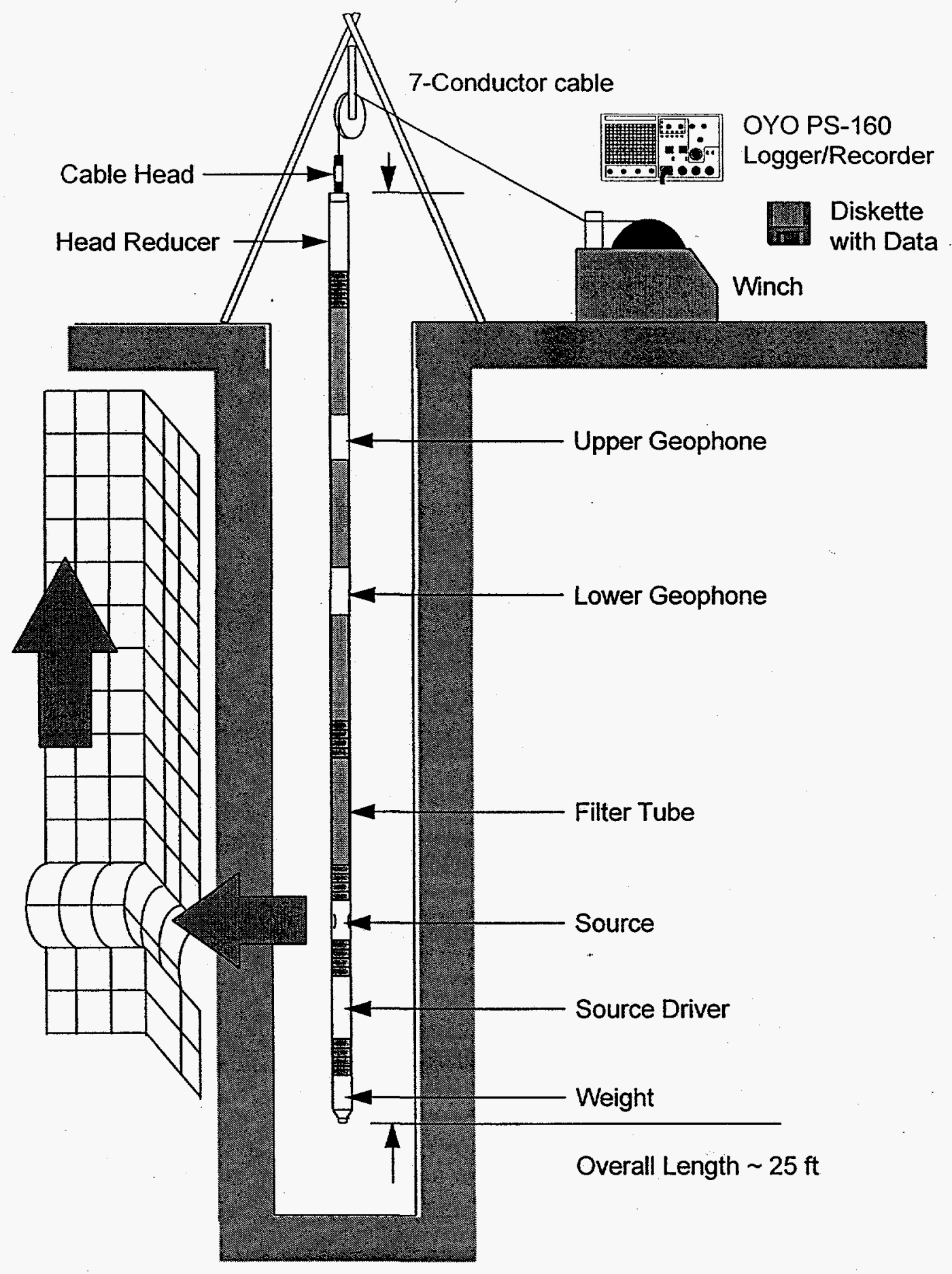

Figure 1. Concept illustration of P-S logging system 


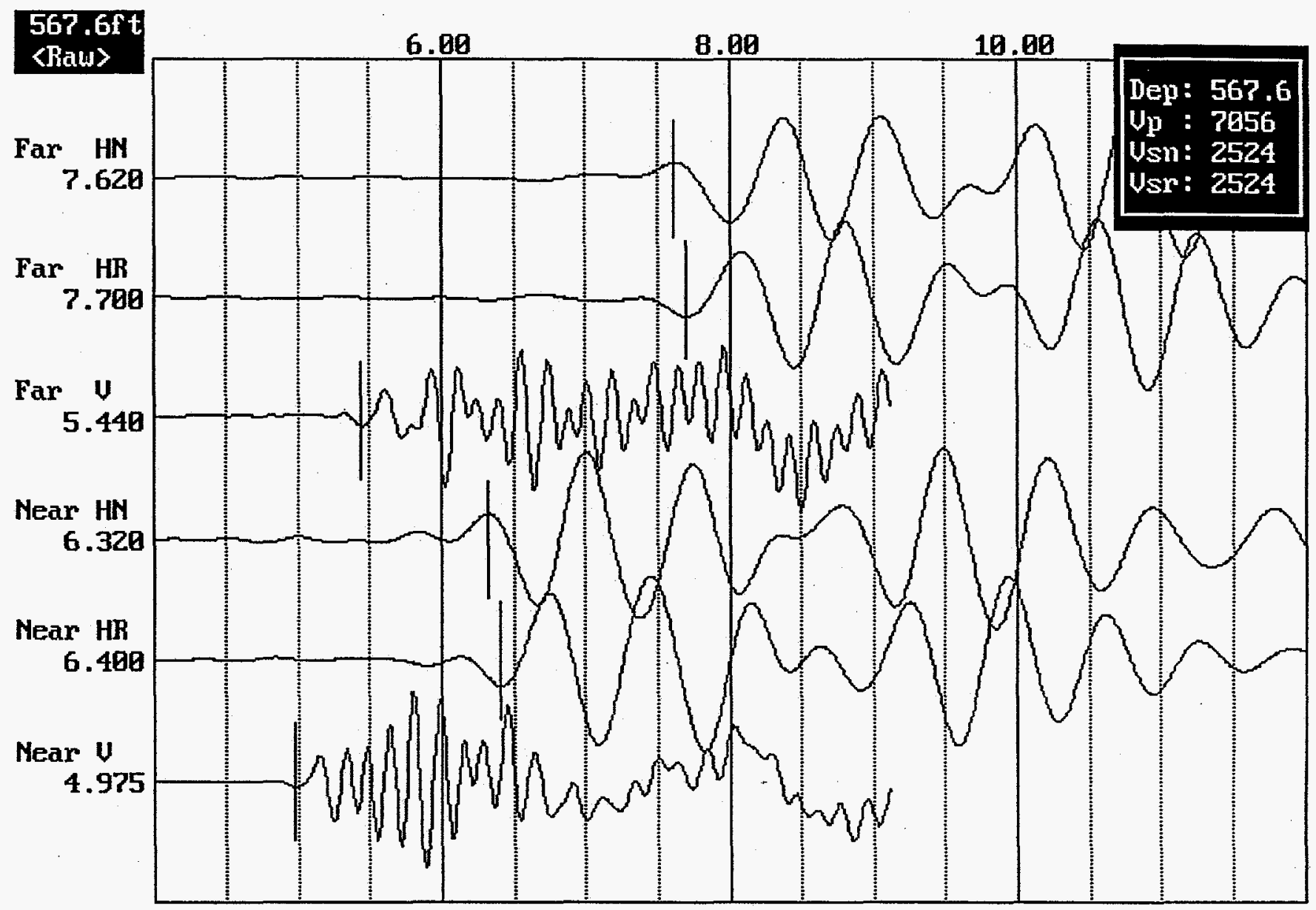

Figure 2. Filtered ( $2000 \mathrm{~Hz}$ lowpass) $567.6 \mathrm{ft}$ record from GCB-1 


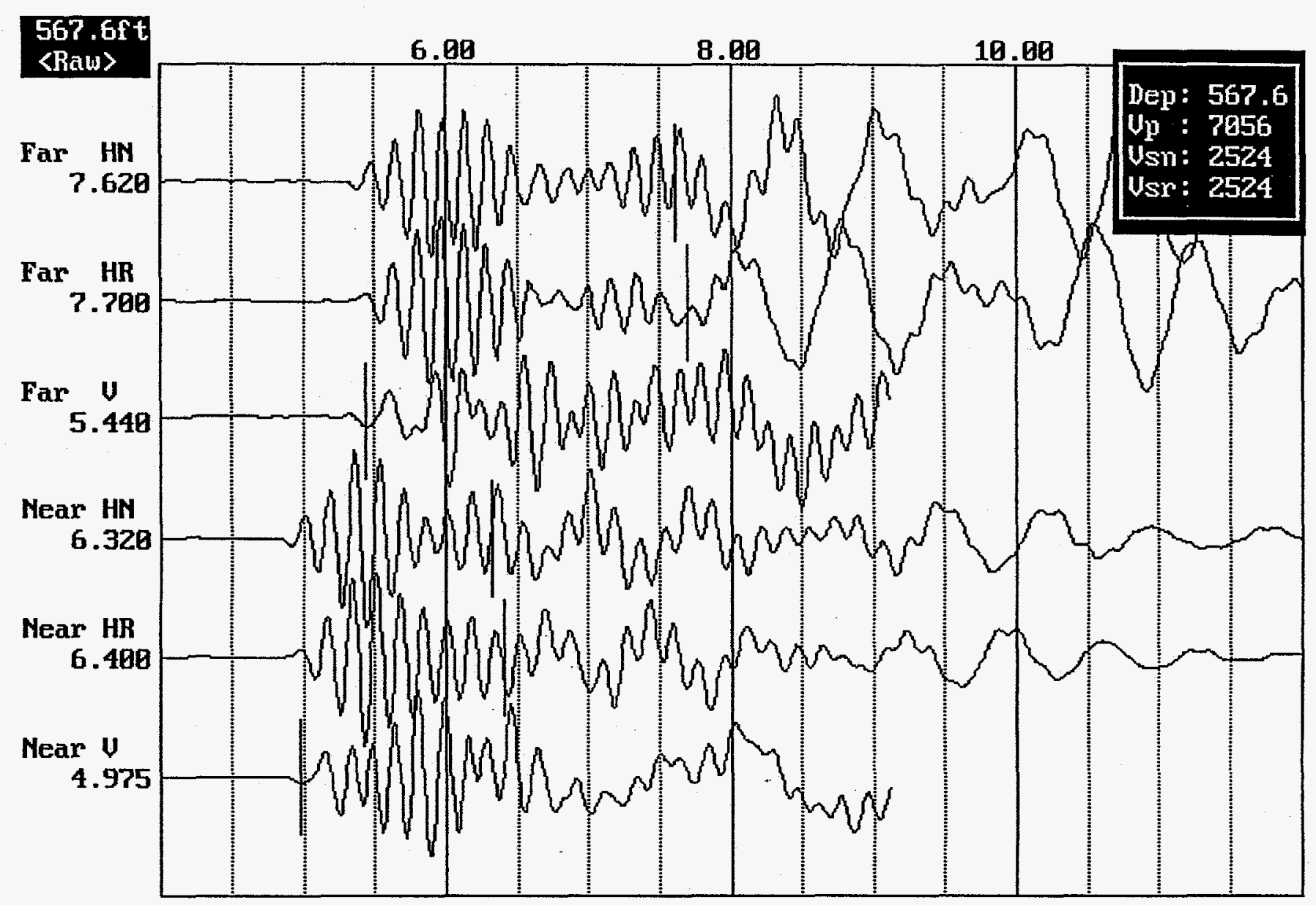

Figure 3. Unfiltered $567.6 \mathrm{ft}$ record from GCB-1 
SRS BOREHOLE GCB-1 OYO SUSPENSION LOGGING

P - AND S-WAVE VELOCITIES, DATA COLLECTED SEPTEMBER 4, 1996

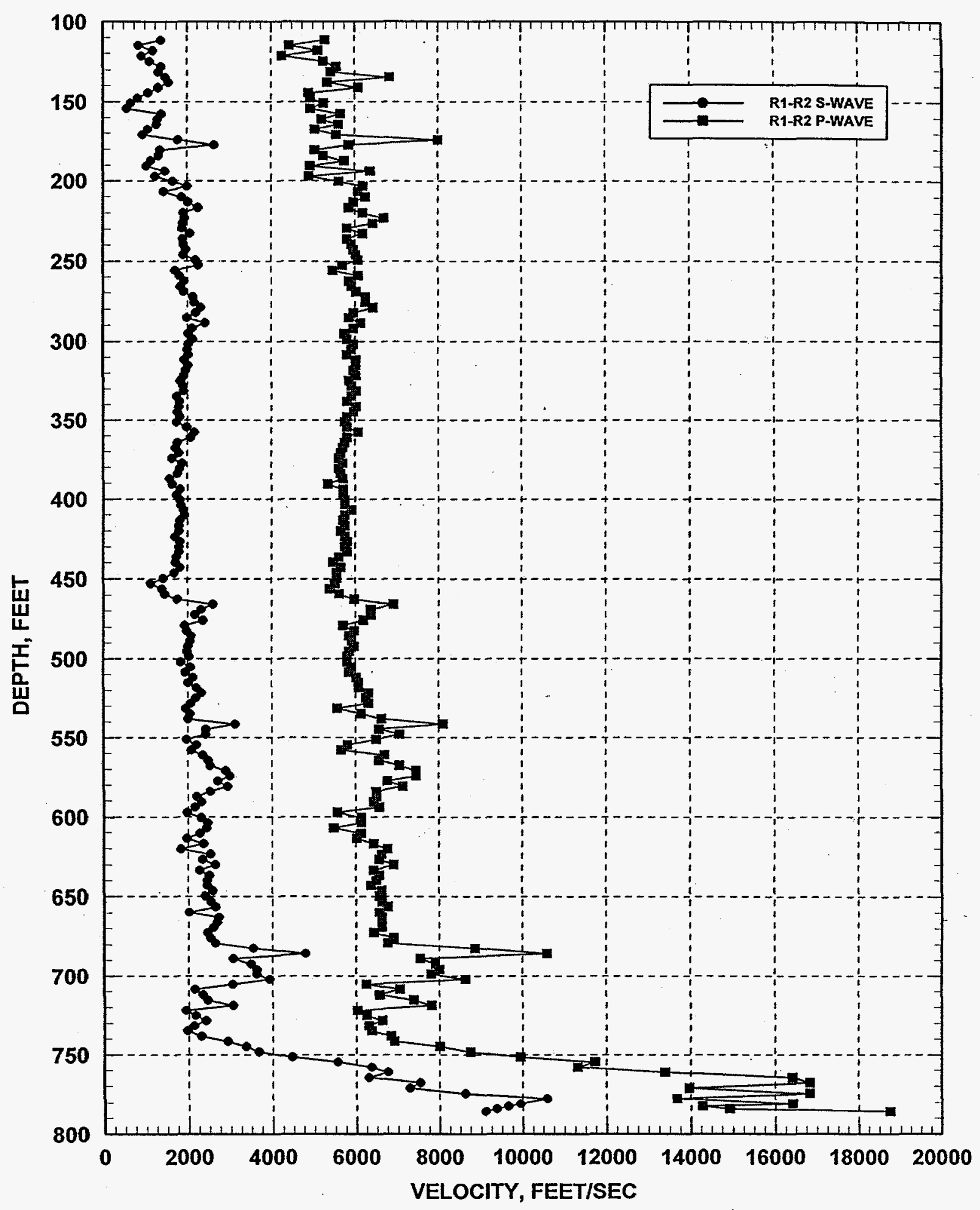

Figure 4. GCB-1 Suspension R1 - R2 P-and $\mathrm{S}_{\mathrm{H}}$-wave velocities 


\section{SRS BOREHOLE GCB-1 OYO SUSPENSION LOGGING}

S-WAVE VELOCITIES, DATA COLLECTED SEPTEMBER 4, 1996

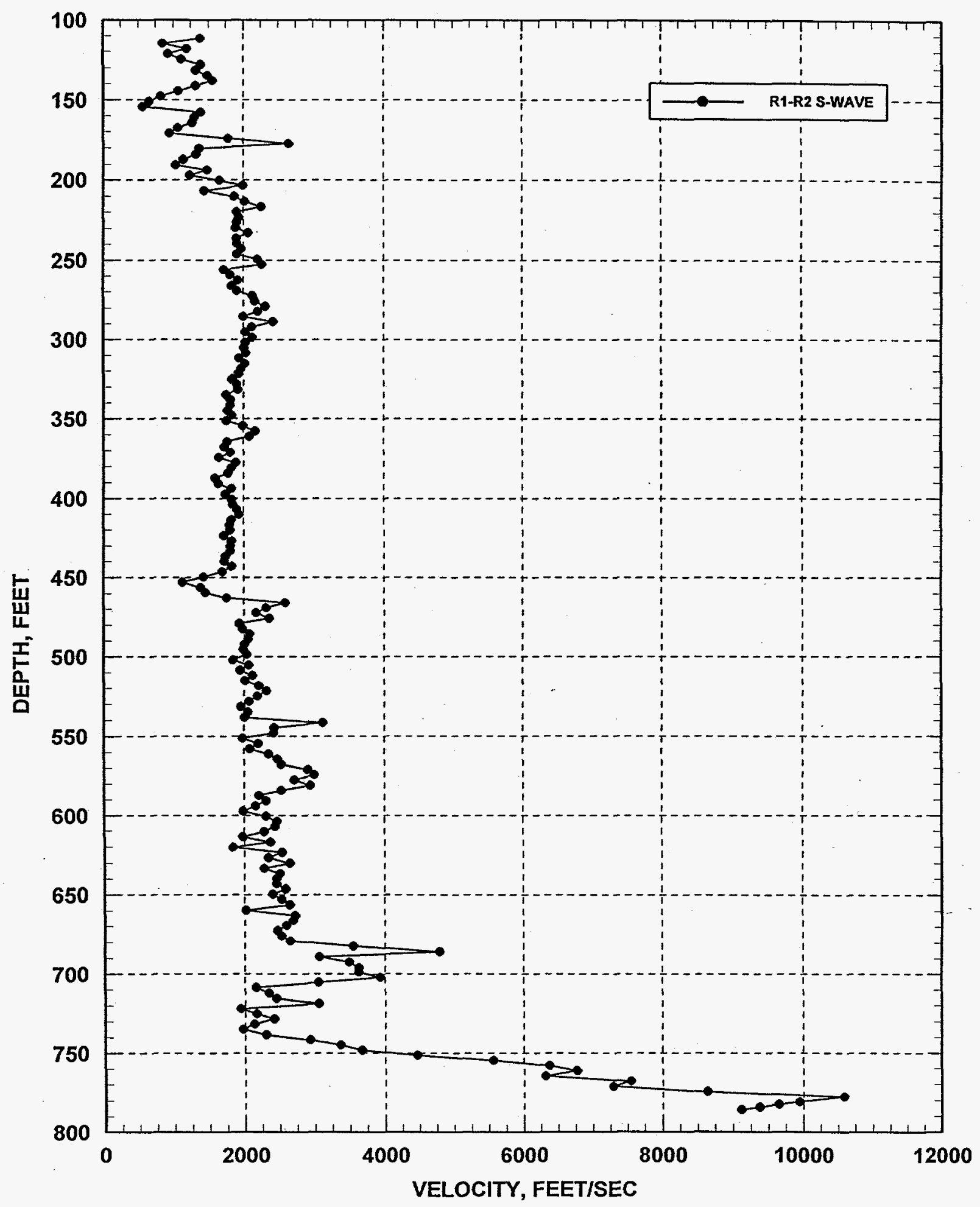

Figure 5. GCB-1 Suspension R1 - R2 $S_{H}$-wave velocities 


\begin{tabular}{|c|c|c|c|c|c|c|c|c|}
\hline \multirow[b]{2}{*}{$\begin{array}{l}\text { Depth, } \\
\text { (feet) }\end{array}$} & \multicolumn{6}{|c|}{ Pick Times } & \multicolumn{2}{|c|}{ Velocity } \\
\hline & $\begin{array}{l}\text { Far-Hn, } \\
\text { (millisec) }\end{array}$ & $\begin{array}{l}\text { Far-Hr, } \\
\text { (millisec) }\end{array}$ & $\begin{array}{c}\text { Far-V, } \\
\text { (millisec) }\end{array}$ & $\begin{array}{l}\text { Near-Hn, } \\
\text { (millisec) }\end{array}$ & $\begin{array}{l}\text { Near-Hr, } \\
\text { (millisec) }\end{array}$ & $\begin{array}{c}\text { Near-V, } \\
\text { (millisec) }\end{array}$ & $\begin{array}{c}\text { Vs, } \\
\text { (ft/sec) }\end{array}$ & $\begin{array}{c}\begin{array}{c}V p \\
(\mathrm{ft} / \mathrm{sec})\end{array}\end{array}$ \\
\hline 111.6 & 14.44 & 14.50 & 6.44 & 12.08 & 12.12 & 5.82 & 1384 & 5292 \\
\hline 114.8 & 15.08 & 15.18 & 6.36 & 11.26 & 11.30 & 5.62 & 852 & 4434 \\
\hline 118.1 & 13.46 & 13.56 & 6.19 & 10.70 & 10.82 & 5.55 & 1193 & 5126 \\
\hline 121.4 & 13.12 & 13.10 & 6.23 & 9.56 & 9.60 & 5.46 & 929 & 4261 \\
\hline 124.7 & 11.94 & 12.00 & 6.06 & 8.94 & 9.12 & 5.44 & 1116 & 5249 \\
\hline 128.0 & 11.54 & 11.72 & 6.07 & 9.22 & 9.32 & 5.48 & 1390 & 5561 \\
\hline 131.2 & 11.90 & 11.96 & 6.10 & 9.38 & 9.50 & 5.49 & 1318 & 5423 \\
\hline 134.5 & 12.40 & 12.48 & 6.00 & 10.18 & 10.28 & 5.52 & 1485 & 6835 \\
\hline 137.8 & 12.80 & 12.92 & 6.07 & 10.70 & 10.80 & 5.45 & 1555 & 5335 \\
\hline 141.1 & 14.36 & 14.50 & 5.98 & 11.92 & 11.96 & 5.44 & 1318 & 6076 \\
\hline 144.4 & 16.12 & 16.20 & 6.12 & 13.06 & 13.14 & 5.45 & 1072 & 4897 \\
\hline 147.6 & 18.04 & 18.16 & 6.14 & 14.06 & 14.18 & 5.48 & 824 & 4934 \\
\hline 150.9 & 18.52 & 18.56 & 6.11 & 13.56 & 13.54 & 5.49 & 657 & 5249 \\
\hline 154.2 & 16.06 & 16.14 & 6.09 & 10.22 & 10.40 & 5.43 & 567 & 4934 \\
\hline 157.5 & 11.46 & 11.58 & 6.04 & 9.02 & 9.30 & 5.46 & 1390 & 5657 \\
\hline 160.8 & 11.86 & 11.94 & 6.02 & 9.32 & 9.44 & 5.39 & 1302 & 5208 \\
\hline 164.0 & 11.88 & 11.96 & 5.92 & 9.28 & 9.40 & 5.33 & 1272 & 5608 \\
\hline 167.3 & 11.22 & 11.36 & 5.89 & 8.14 & 8.30 & 5.24 & 1069 & 5047 \\
\hline 170.6 & 11.40 & 11.38 & 5.80 & 7.88 & 8.00 & 5.21 & 951 & 5561 \\
\hline 173.9 & 9.62 & 9.68 & 5.63 & 7.76 & 7.86 & 5.22 & 1783 & 8002 \\
\hline 177.2 & 10.22 & 10.30 & 5.85 & 8.96 & 9.08 & 5.29 & 2646 & 5859 \\
\hline 180.1 & 11.42 & 11.48 & 6.03 & 9.02 & 9.08 & 5.38 & 1367 & 5047 \\
\hline 183.7 & 12.26 & 12.34 & 6.08 & 9.76 & 9.88 & 5.46 & 1323 & 5249 \\
\hline 187.0 & 12.90 & 13.60 & 5.96 & 10.02 & 10.74 & 5.39 & 1143 & 5756 \\
\hline 190.3 & 12.08 & 12.16 & 6.02 & 8.96 & 8.94 & 5.35 & 1035 & 4934 \\
\hline 193.6 & 10.92 & 11.00 & 5.91 & 8.70 & 8.78 & 5.40 & 1478 & 6371 \\
\hline 196.9 & 10.88 & 11.00 & 6.02 & 8.26 & 8.32 & 5.35 & 1238 & 4897 \\
\hline 200.1 & 10.42 & 10.54 & 5.90 & 8.46 & 8.54 & 5.31 & 1657 & 5608 \\
\hline 203.4 & 10.03 & 10.13 & 5.88 & 8.37 & 8.50 & 5.35 & 1994 & 6190 \\
\hline 206.7 & 9.92 & 9.98 & 5.90 & 7.68 & 7.67 & 5.36 & 1442 & 6076 \\
\hline 210.0 & 9.29 & 9.38 & 5.76 & 7.52 & 7.64 & 5.24 & 1869 & 6249 \\
\hline 213.3 & 9.21 & 9.32 & 5.72 & 7.59 & 7.69 & 5.17 & 2019 & 5965 \\
\hline 216.5 & 9.10 & 9.22 & 5.67 & 7.65 & 7.76 & 5.11 & 2255 & 5859 \\
\hline 219.8 & 9.29 & 9.44 & 5.64 & 7.59 & 7.70 & 5.11 & 1907 & 6190 \\
\hline 223.1 & 9.30 & 10.04 & 5.75 & 7.67 & 8.28 & 5.26 & 1936 & 6696 \\
\hline 226.4 & 9.18 & 9.32 & 5.81 & 7.62 & 7.42 & 5.30 & 1896 & 6433 \\
\hline 229.7 & 9.94 & 9.96 & 5.91 & 8.18 & 8.24 & 5.34 & 1886 & 5807 \\
\hline 232.9 & 9.70 & 9.78 & 5.89 & 8.10 & 8.20 & 5.36 & 2063 & 6190 \\
\hline 236.2 & 9.70 & 9.66 & 5.78 & 7.92 & 7.98 & 5.21 & 1896 & 5807 \\
\hline 239.5 & 9.64 & 9.64 & 5.74 & 7.86 & 7.98 & 5.19 & 1907 & 5911 \\
\hline 242.8 & 9.54 & 9.60 & 5.73 & 7.86 & 7.94 & 5.18 & 1965 & 5965 \\
\hline 246.1 & 10.24 & 10.94 & 5.87 & 8.48 & 9.26 & 5.33 & 1907 & 6020 \\
\hline 249.3 & 9.96 & 10.00 & 5.94 & 8.46 & 8.52 & 5.40 & 2202 & 6076 \\
\hline 252.6 & 10.14 & 10.98 & 6.03 & 8.64 & 9.58 & 5.46 & 2263 & 5706 \\
\hline 255.9 & 10.12 & 11.22 & 6.03 & 8.28 & 9.24 & 5.43 & 1718 & 5468 \\
\hline 259.2 & 9.92 & 10.10 & 5.80 & 8.12 & 8.28 & 5.26 & 1813 & 6076 \\
\hline 262.5 & 9.88 & 9.90 & 5.79 & 8.14 & 8.22 & 5.23 & 1919 & 5859 \\
\hline 265.8 & 9.60 & 9.64 & 5.75 & 7.76 & 7.90 & 5.20 & 1833 & 5911 \\
\hline 269.0 & 9.06 & 9.08 & 5.70 & 7.32 & 7.38 & 5.16 & 1907 & 6020 \\
\hline 272.3 & 8.82 & 8.92 & 5.67 & 7.28 & 7.38 & 5.15 & 2130 & 6249 \\
\hline 275.6 & 8.84 & 8.92 & 5.69 & 7.32 & 7.40 & 5.17 & 2158 & 6249 \\
\hline 278.9 & 8.73 & 8.80 & 5.66 & 7.30 & 7.39 & 5.15 & 2310 & 6433 \\
\hline 282.2 & 8.76 & 8.85 & 5.68 & 7.28 & 7.35 & 5.13 & 2202 & 5965 \\
\hline
\end{tabular}

Table 1. GCB-1 Suspension R1-R2 depth, pick times, and velocities 


\begin{tabular}{|c|c|c|c|c|c|c|c|c|}
\hline \multirow[b]{2}{*}{$\begin{array}{l}\text { Depth, } \\
\text { (feet) }\end{array}$} & \multicolumn{6}{|c|}{ Pick Times } & \multicolumn{2}{|c|}{ Velocity } \\
\hline & $\begin{array}{c}\text { Far-Hn, } \\
\text { (millisec) }\end{array}$ & $\begin{array}{c}\text { Far-Hr, } \\
\text { (millisec) }\end{array}$ & $\begin{array}{c}\text { Far-V, } \\
\text { (millisec) }\end{array}$ & $\begin{array}{l}\text { Near-Hn, } \\
\text { (millisec) }\end{array}$ & $\begin{array}{l}\text { Near-Hr, } \\
\text { (millisec) }\end{array}$ & $\begin{array}{l}\text { Near-V, } \\
\text { (millisec) }\end{array}$ & $\begin{array}{c}\text { Vs, } \\
\text { (ft/sec) }\end{array}$ & $\begin{array}{c}V_{p} \\
(\mathrm{f} / \mathrm{sec})\end{array}$ \\
\hline 285.4 & 8.77 & 8.87 & 5.68 & 7.13 & 7.22 & 5.12 & 1994 & 5859 \\
\hline 288.7 & 8.78 & 8.87 & 5.68 & 7.43 & 7.51 & 5.15 & 2421 & 6132 \\
\hline 292.0 & 9.08 & 9.17 & 5.72 & 7.53 & 7.62 & 5.17 & 2117 & 5965 \\
\hline 295.3 & 9.15 & 9.25 & 5.73 & 7.53 & 7.63 & 5.16 & 2025 & 5756 \\
\hline 298.6 & 9.10 & 9.22 & 5.70 & 7.56 & 7.67 & 5.13 & 2124 & 5807 \\
\hline 301.8 & 9.21 & 9.33 & 5.70 & 7.59 & 7.71 & 5.15 & 2025 & 5965 \\
\hline 305.1 & 9.22 & 9.32 & 5.72 & 7.59 & 7.68 & 5.17 & 2007 & 5911 \\
\hline 308.4 & 9.18 & 9.29 & 5.74 & 7.57 & 7.67 & 5.17 & 2031 & 5807 \\
\hline 311.7 & 9.23 & 9.33 & 5.75 & 7.54 & 7.63 & 5.21 & 1936 & 6020 \\
\hline 315.0 & 9.24 & 9.32 & 5.76 & 7.62 & 7.69 & 5.22 & 2019 & 6020 \\
\hline 318.2 & 9.33 & 9.39 & 5.77 & 7.66 & 7.72 & 5.22 & 1965 & 5965 \\
\hline 321.5 & 9.46 & 9.53 & 5.77 & 7.76 & 7.82 & 5.23 & 1924 & 6020 \\
\hline 324.8 & 9.56 & 9.63 & 5.69 & 7.77 & 7.84 & 5.13 & 1833 & 5859 \\
\hline 328.1 & 9.53 & 9.61 & 5.69 & 7.80 & 7.88 & 5.13 & 1896 & 5911 \\
\hline 331.4 & 9.68 & 9.77 & 5.69 & 7.97 & 8.05 & 5.15 & 1913 & 6020 \\
\hline 334.7 & 9.80 & 9.87 & 5.72 & 7.93 & 7.99 & 5.17 & 1750 & 5911 \\
\hline 337.9 & 9.75 & 9.82 & 5.71 & 7.94 & 8.01 & 5.15 & 1813 & 5807 \\
\hline 341.2 & 9.73 & 9.86 & 5.69 & 7.91 & 8.04 & 5.14 & 1803 & 6020 \\
\hline 344.5 & 9.79 & 9.88 & 5.71 & 7.93 & 8.03 & 5.16 & 1769 & 5965 \\
\hline 347.8 & 9.73 & 9.80 & 5.84 & 7.94 & 8.00 & 5.27 & 1828 & 5807 \\
\hline 351.1 & 9.57 & 9.69 & 5.72 & 7.70 & 7.82 & 5.15 & 1754 & 5756 \\
\hline 354.3 & 9.45 & 9.55 & 5.71 & 7.81 & 7.89 & 5.14 & 1988 & 5807 \\
\hline 357.6 & 9.56 & 9.62 & 5.73 & 8.03 & 8.12 & 5.19 & 2166 & 6076 \\
\hline 360.9 & 9.61 & 9.65 & 5.69 & 8.04 & 8.07 & 5.13 & 2083 & 5807 \\
\hline 364.2 & 9.84 & 9.92 & 5.76 & 7.98 & 8.06 & 5.19 & 1764 & 5756 \\
\hline 367.5 & 9.78 & 9.84 & 5.78 & 7.87 & 7.94 & 5.20 & 1722 & 5706 \\
\hline 370.7 & 9.76 & 9.82 & 5.87 & 7.95 & 7.99 & 5.29 & 1803 & 5657 \\
\hline 374.0 & 9.77 & 9.86 & 5.86 & 7.76 & 7.87 & 5.28 & 1640 & 5608 \\
\hline 377.3 & 9.47 & 9.59 & 5.83 & 7.73 & 7.84 & 5.26 & 1880 & 5706 \\
\hline 380.6 & 9.94 & 10.01 & 5.77 & 8.14 & 8.20 & 5.18 & 1818 & 5608 \\
\hline 383.9 & 10.10 & 10.10 & 5.78 & 8.30 & 8.20 & 5.20 & 1773 & 5657 \\
\hline 387.1 & 10.19 & 10.22 & 5.80 & 8.10 & 8.18 & 5.22 & 1589 & 5706 \\
\hline 390.4 & 10.00 & 10.07 & 5.80 & 7.99 & 8.06 & 5.18 & 1632 & 5335 \\
\hline 393.7 & 9.80 & 9.86 & 5.75 & 7.99 & 8.06 & 5.17 & 1818 & 5706 \\
\hline 397.0 & 9.77 & 9.84 & 5.74 & 7.89 & 7.94 & 5.16 & 1736 & 5706 \\
\hline 400.3 & 9.54 & 9.61 & 5.72 & 7.72 & 7.83 & 5.15 & 1823 & 5756 \\
\hline 403.5 & 9.60 & 9.66 & 5.73 & 7.81 & 7.89 & 5.16 & 1843 & 5756 \\
\hline 406.8 & 9.68 & 9.75 & 5.74 & 7.95 & 8.03 & 5.19 & 1902 & 5911 \\
\hline 410.1 & 9.67 & 9.75 & 5.74 & 7.97 & 8.05 & 5.17 & 1930 & 5756 \\
\hline 413.4 & 9.86 & 9.92 & 5.75 & 8.06 & 8.12 & 5.17 & 1823 & 5706 \\
\hline 416.7 & 9.86 & 9.93 & 5.75 & 8.03 & 8.11 & 5.18 & 1798 & 5756 \\
\hline 420.0 & 9.89 & 9.97 & 5.74 & 8.06 & 8.15 & 5.16 & 1798 & 5657 \\
\hline 423.2 & 9.90 & 9.99 & 5.73 & 7.98 & 8.08 & 5.16 & 1713 & 5756 \\
\hline 426.5 & 9.84 & 9.92 & 5.73 & 8.03 & 8.12 & 5.17 & 1818 & 5807 \\
\hline 429.8 & 9.76 & 9.84 & 5.76 & 7.94 & 8.02 & 5.19 & 1803 & 5756 \\
\hline 433.1 & 10.00 & 10.06 & 5.79 & 8.18 & 8.24 & 5.23 & 1803 & 5807 \\
\hline 436.4 & 10.06 & 10.12 & 5.81 & 8.16 & 8.24 & 5.23 & 1736 & 5608 \\
\hline 439.6 & 10.22 & 10.26 & 5.80 & 8.30 & 8.36 & 5.20 & 1718 & 5468 \\
\hline 442.9 & 10.46 & 10.52 & 5.80 & 8.66 & 8.72 & 5.22 & 1823 & 5657 \\
\hline 446.2 & 11.20 & 11.28 & 5.81 & 9.26 & 9.34 & 5.22 & 1691 & 5561 \\
\hline 449.5 & 11.18 & 11.22 & 5.84 & 8.84 & 8.96 & 5.25 & 1426 & 5561 \\
\hline 452.8 & 11.86 & 11.96 & 5.84 & 8.94 & 9.04 & 5.24 & 1124 & 5514 \\
\hline
\end{tabular}

Table 1 continued. GCB-1 Suspension R1-R2 depth, pick times, and velocities 


\begin{tabular}{|c|c|c|c|c|c|c|c|c|}
\hline \multirow[b]{2}{*}{$\begin{array}{l}\text { Depth, } \\
\text { (feet) }\end{array}$} & \multicolumn{6}{|c|}{ Pick Times } & \multicolumn{2}{|c|}{ Velocity } \\
\hline & $\begin{array}{c}\text { Far-Hn, } \\
\text { (millisec) }\end{array}$ & $\begin{array}{c}\text { Far-Hr, } \\
\text { (millisec) }\end{array}$ & $\begin{array}{c}\text { Far-V, } \\
\text { (millisec) }\end{array}$ & $\begin{array}{l}\text { Near-Hn, } \\
\text { (millisec) }\end{array}$ & $\begin{array}{l}\text { Near-Hr, } \\
\text { (millisec) }\end{array}$ & $\begin{array}{l}\text { Near-V, } \\
\text { (millisec) }\end{array}$ & $\begin{array}{c}\mathrm{Vs} \\
\text { (ft/sec) }\end{array}$ & $\begin{array}{c}V_{p} \\
\text { (ft/sec) }\end{array}$ \\
\hline 456.0 & 10.54 & 10.64 & 5.80 & 8.18 & 8.26 & 5.19 & 1384 & 5378 \\
\hline 459.3 & 9.46 & 9.53 & 5.68 & 7.19 & 7.29 & 5.10 & 1455 & 5608 \\
\hline 462.6 & 8.75 & 8.83 & 5.63 & 6.88 & 6.96 & 5.08 & 1754 & 5965 \\
\hline 465.9 & 8.26 & 8.36 & 5.58 & 7.00 & 7.09 & 5.11 & 2594 & 6907 \\
\hline 469.2 & 8.54 & 8.63 & 5.63 & 7.13 & 7.21 & 5.11 & 2319 & 6371 \\
\hline 472.4 & 8.80 & 8.88 & 5.65 & 7.29 & 7.37 & 5.14 & 2173 & 6371 \\
\hline 475.7 & 8.93 & 9.01 & 5.70 & 7.53 & 7.63 & 5.17 & 2360 & 6190 \\
\hline 479.0 & 9.19 & 9.25 & 5.73 & 7.49 & 7.55 & 5.15 & 1930 & 5706 \\
\hline 482.3 & 9.09 & 9.15 & 5.70 & 7.42 & 7.50 & 5.15 & 1976 & 5965 \\
\hline 485.6 & 9.09 & 9.16 & 5.72 & 7.50 & 7.59 & 5.16 & 2076 & 5859 \\
\hline 488.9 & 9.13 & 9.25 & 5.73 & 7.53 & 7.66 & 5.17 & 2057 & 5911 \\
\hline 492.1 & 9.15 & 9.23 & 5.72 & 7.52 & 7.59 & 5.17 & 2007 & 5965 \\
\hline 495.4 & 9.07 & 9.15 & 5.71 & 7.42 & 7.50 & 5.15 & 1988 & 5859 \\
\hline 498.7 & 9.20 & 9.29 & 5.74 & 7.59 & 7.68 & 5.17 & 2038 & 5807 \\
\hline 502.0 & 9.25 & 9.34 & 5.75 & 7.48 & 7.55 & 5.18 & 1843 & 5807 \\
\hline 505.3 & 9.17 & 9.27 & 5.78 & 7.59 & 7.68 & 5.23 & 2070 & 5911 \\
\hline 508.5 & 9.12 & 9.21 & 5.69 & 7.45 & 7.51 & 5.13 & 1947 & 5859 \\
\hline 511.8 & 9.01 & 9.12 & 5.66 & 7.47 & 7.57 & 5.12 & 2124 & 6020 \\
\hline 515.1 & 8.96 & 9.05 & 5.65 & 7.33 & 7.42 & 5.11 & 2013 & 6076 \\
\hline 518.4 & 8.92 & 8.99 & 5.65 & 7.44 & 7.50 & 5.11 & 2209 & 6076 \\
\hline 521.7 & 9.01 & 9.11 & 5.64 & 7.61 & 7.68 & 5.12 & 2319 & 6309 \\
\hline 524.9 & 9.11 & 9.16 & 5.64 & 7.61 & 7.66 & 5.12 & 2187 & 6249 \\
\hline 528.2 & 9.12 & 9.20 & 5.72 & 7.53 & 7.62 & 5.20 & 2070 & $6309^{\circ}$ \\
\hline 531.5 & 8.90 & 8.99 & 5.80 & 7.22 & 7.31 & 5.21 & 1953 & 5561 \\
\hline 534.8 & 8.30 & 8.41 & 5.64 & 6.70 & 6.81 & 5.11 & 2051 & 6132 \\
\hline 538.1 & 8.14 & 8.24 & 5.58 & 6.53 & 6.58 & 5.09 & 2007 & 6628 \\
\hline 541.3 & 8.11 & 8.20 & 5.56 & 7.08 & 7.13 & 5.15 & 3125 & 8101 \\
\hline 544.6 & 8.49 & 8.56 & 5.63 & 7.14 & 7.21 & 5.13 & 2430 & 6562 \\
\hline 547.9 & 8.72 & 8.78 & 5.64 & 7.36 & 7.43 & 5.17 & 2421 & 7056 \\
\hline 551.2 & 8.84 & 8.89 & 5.68 & 7.16 & 7.25 & 5.17 & 1976 & 6497 \\
\hline 554.5 & 8.50 & 8.59 & 5.66 & 7.01 & 7.10 & 5.09 & 2202 & 5807 \\
\hline 557.7 & 8.33 & 8.42 & 5.63 & 6.75 & 6.85 & 5.05 & 2083 & 5657 \\
\hline 561.0 & 8.02 & 8.11 & 5.54 & 6.62 & 6.72 & 5.05 & 2352 & 6696 \\
\hline 564.3 & 7.72 & 7.81 & 5.49 & 6.40 & 6.48 & 4.99 & 2476 & 6562 \\
\hline 567.6 & 7.62 & 7.70 & 5.44 & 6.32 & 6.40 & 4.98 & 2524 & 7056 \\
\hline 570.9 & 7.45 & 7.52 & 5.42 & 6.32 & 6.39 & 4.98 & 2903 & 7456 \\
\hline 574.2 & 7.51 & 7.63 & 5.43 & 6.42 & 6.53 & 4.99 & 2996 & 7456 \\
\hline 577.4 & 7.83 & 7.90 & 5.53 & 6.62 & 6.69 & 5.04 & 2711 & 6765 \\
\hline .580 .7 & 8.10 & 8.19 & 5.54 & 6.98 & 7.08 & 5.08 & 2942 & 7132 \\
\hline 584.0 & 8.44 & 8.52 & 5.58 & 7.12 & 7.24 & 5.08 & 2524 & 6497 \\
\hline 587.3 & 8.71 & 8.82 & 5.59 & 7.23 & 7.33 & 5.08 & 2209 & 6497 \\
\hline 590.6 & 8.73 & 8.81 & 5.62 & 7.30 & 7.40 & 5.11 & 2310 & 6433 \\
\hline 593.8 & 8.77 & 8.84 & 5.61 & 7.25 & 7.32 & 5.11 & 2158 & 6562 \\
\hline 597.1 & 8.85 & 8.89 & 5.72 & 7.17 & 7.26 & 5.13 & 1982 & 5561 \\
\hline 600.4 & 8.70 & 8.79 & 5.72 & 7.27 & 7.38 & 5.19 & 2310 & 6132 \\
\hline 603.7 & 8.41 & 8.43 & 5.72 & 7.06 & 7.12 & 5.18 & 2467 & 6132 \\
\hline 607.0 & 8.63 & 8.69 & 5.72 & 7.27 & 7.36 & 5.12 & 2439 & 5468 \\
\hline 610.2 & 8.82 & 8.88 & 5.65 & 7.39 & 7.44 & 5.12 & 2286 & 6132 \\
\hline 613.5 & 8.61 & 8.69 & 5.62 & 6.94 & 7.04 & 5.07 & 1976 & 6020 \\
\hline 616.8 & 8.51 & 8.57 & 5.57 & 7.11 & 7.20 & 5.06 & 2369 & 6433 \\
\hline 620.1 & 8.80 & 8.88 & 5.56 & 7.01 & $7.09^{\circ}$ & 5.08 & 1833 & 6765 \\
\hline 623.4 & 8.23 & 8.32 & 5.51 & 6.94 & 7.02 & 5.01 & 2533 & 6628 \\
\hline 626.6 & 8.31 & 8.38 & 5.52 & 6.91 & 6.98 & 5.02 & 2343 & 6562 \\
\hline
\end{tabular}

Table 1 continued. GCB-1 Suspension R1-R2 depth, pick times, and velocities 


\begin{tabular}{|c|c|c|c|c|c|c|c|c|}
\hline \multirow[b]{2}{*}{$\begin{array}{l}\text { Depth, } \\
\text { (feet) }\end{array}$} & \multicolumn{6}{|c|}{ Pick Times } & \multicolumn{2}{|c|}{ Velocity } \\
\hline & $\begin{array}{l}\text { Far-Hn, } \\
\text { (millisec) }\end{array}$ & $\begin{array}{c}\text { Far- } \mathrm{Hr}, \\
\text { (millisec) }\end{array}$ & $\begin{array}{c}\text { Far-V, } \\
\text { (millisec) }\end{array}$ & $\begin{array}{l}\text { Near-Hn, } \\
\text { (millisec) }\end{array}$ & $\begin{array}{l}\text { Near-Hr, } \\
\text { (millisec) }\end{array}$ & $\begin{array}{l}\text { Near-V, } \\
\text { (millisec) }\end{array}$ & $\begin{array}{c}\text { Vs, } \\
\text { (ft/sec) }\end{array}$ & $\begin{array}{c}V p \\
(\mathrm{ft} / \mathrm{sec})\end{array}$ \\
\hline 629.9 & 8.22 & 8.30 & 5.56 & 6.98 & 7.06 & 5.09 & 2646 & 6907 \\
\hline 633.2 & 8.24 & 8.33 & 5.59 & 6.81 & 6.88 & 5.08 & 2278 & 6433 \\
\hline 636.5 & 8.15 & 8.24 & 5.58 & 6.84 & 6.93 & 5.08 & 2504 & 6562 \\
\hline 639.8 & 8.17 & 8.25 & 5.61 & 6.84 & 6.91 & 5.10 & 2458 & 6497 \\
\hline 643.0 & 8.10 & 8.14 & 5.60 & 6.76 & 6.81 & 5.08 & 2458 & 6371 \\
\hline 646.3 & 7.98 & 8.04 & 5.65 & 6.71 & 6.77 & 5.16 & 2583 & 6628 \\
\hline 649.6 & 8.09 & 8.15 & 5.54 & 6.73 & 6.78 & 5.04 & 2404 & 6562 \\
\hline 652.9 & 8.06 & 8.12 & 5.55 & 6.77 & 6.82 & 5.05 & 2533 & 6628 \\
\hline 656.2 & 8.08 & 8.14 & 5.49 & 6.85 & 6.89 & 5.00 & 2646 & 6765 \\
\hline 659.5 & 8.35 & 8.46 & 5.53 & 6.74 & 6.82 & 5.03 & 2019 & 6562 \\
\hline 662.7 & 7.95 & 8.01 & 5.54 & 6.75 & 6.80 & 5.04 & 2723 & 6628 \\
\hline 666.0 & 8.08 & 8.13 & 5.57 & 6.87 & 6.90 & 5.07 & 2689 & 6628 \\
\hline 669.3 & 7.98 & 7.90 & 5.67 & 6.73 & 6.62 & 5.18 & 2594 & 6628 \\
\hline 672.6 & 8.00 & 8.07 & 5.53 & 6.66 & 6.75 & 5.02 & 2467 & 6433 \\
\hline 675.9 & 7.21 & 7.30 & 5.37 & 5.90 & 6.01 & 4.90 & 2524 & 6907 \\
\hline 679.1 & 6.86 & 6.93 & 5.31 & 5.62 & 5.69 & 4.82 & 2646 & 6765 \\
\hline 682.4 & 6.59 & 6.59 & 5.14 & 5.67 & 5.66 & 4.77 & 3547 & 8867 \\
\hline 685.7 & 6.57 & 6.64 & 5.14 & 5.89 & 5.95 & 4.83 & 4790 & 10583 \\
\hline 689.0 & 6.85 & 6.90 & 5.33 & 5.79 & 5.82 & 4.90 & 3066 & 7542 \\
\hline 692.3 & 6.71 & 6.80 & 5.30 & 5.77 & 5.86 & 4.89 & 3490 & 7906 \\
\hline 695.9 & 6.66 & 6.74 & 5.31 & 5.76 & 5.83 & 4.90 & 3625 & 8002 \\
\hline 698.8 & 7.14 & 7.21 & 5.44 & 6.23 & 6.31 & 5.02 & 3625 & 7812 \\
\hline 702.1 & 7.82 & 7.89 & 5.53 & 6.98 & 7.06 & 5.15 & 3929 & 8634 \\
\hline 705.4 & 8.44 & 8.48 & 5.64 & 7.33 & 7.44 & 5.11 & 3052 & 6249 \\
\hline 708.7 & 8.65 & 8.70 & 5.55 & 7.12 & 7.19 & 5.08 & 2158 & 7056 \\
\hline 711.9 & 8.37 & 8.45 & 5.59 & 6.97 & 7.05 & 5.09 & 2343 & 6562 \\
\hline 715.2 & 8.32 & 8.40 & 5.60 & 6.98 & 7.06 & 5.16 & 2448 & 7373 \\
\hline 718.5 & 8.34 & 8.44 & 5.52 & 7.29 & 7.34 & 5.10 & 3052 & 7812 \\
\hline 721.8 & 8.61 & 8.72 & 5.63 & 6.94 & 7.00 & 5.09 & 1936 & 6020 \\
\hline 725.1 & 8.46 & 8.54 & 5.61 & 6.96 & 7.01 & 5.09 & 2166 & 6249 \\
\hline 728.4 & 8.51 & 8.65 & 5.64 & 7.19 & 7.25 & 5.14 & 2412 & 6628 \\
\hline 731.6 & 8.43 & 8.50 & 5.66 & 6.88 & 6.97 & 5.14 & 2130 & 6309 \\
\hline 734.9 & 8.00 & 8.02 & 5.57 & 6.28 & 6.41 & 5.06 & 1970 & 6371 \\
\hline 738.2 & 7.34 & 7.42 & 5.39 & 5.92 & 5.99 & 4.91 & 2302 & 6835 \\
\hline 741.5 & 6.64 & 6.70 & 5.24 & 5.54 & 5.56 & 4.77 & 2929 & 6907 \\
\hline 744.8 & 6.27 & 6.31 & 5.22 & 5.31 & 5.32 & 4.81 & 3365 & 8002 \\
\hline 748.0 & 6.18 & 6.18 & 5.03 & 5.28 & 5.29 & 4.65 & 3666 & 8749 \\
\hline 751.3 & 5.85 & 5.82 & 4.92 & 5.08 & 5.12 & 4.59 & 4464 & 9942 \\
\hline 754.6 & 5.63 & 5.70 & 4.86 & 5.05 & 5.10 & 4.58 & 5561 & 11717 \\
\hline 757.9 & 5,49 & 5.61 & 4.87 & 4.97 & 5.10 & 4.58 & 6371 & 11313 \\
\hline 761.2 & 5.56 & 5.56 & 4.81 & 5.05 & 5.10 & 4.56 & 6765 & 13391 \\
\hline 764.4 & 5.54 & 5.57 & 4.71 & 5.01 & 5.06 & 4.51 & 6309 & 16404 \\
\hline 767.7 & 5.31 & 5.38 & 4.60 & 4.87 & 4.95 & 4.40 & 7542 & 16825 \\
\hline 771.0 & 5.19 & 5.21 & 4.66 & 4.72 & 4.78 & 4.42 & 7291 & 13961 \\
\hline 774.3 & 5.04 & 5.10 & 4.58 & 4.66 & 4.72 & 4.39 & 8634 & 16825 \\
\hline 777.6 & 5.00 & 5.06 & 4.65 & 4.69 & 4.75 & 4.41 & 10583 & 13670 \\
\hline 780.8 & 5.06 & 5.12 & 4.56 & 4.73 & 4.79 & 4.36 & 9942 & 16404 \\
\hline 782.5 & 5.08 & 5.13 & 4.63 & 4.74 & 4.79 & 4.40 & 9650 & 14265 \\
\hline 784.1 & 5.14 & 5.18 & 4.66 & 4.79 & 4.83 & 4.44 & 9374 & 14913 \\
\hline 785.8 & 5.16 & 5.23 & 4.67 & 4.81 & 4.86 & 4.49 & 9113 & 18748 \\
\hline
\end{tabular}

Table 1 continued. GCB-1 Suspension R1-R2 depth, pick times, and velocities 
APPENDIX A

SUSPENSION VELOCITY MEASUREMENT

QUALITY ASSURANCE SUSPENSION SOURCE

TO RECEIVER ANALYSIS RESULTS 
SRS BOREHOLE GCB-1 OYO SUSPENSION LOGGING

P - AND S-WAVE VELOCITIES, DATA COLLECTED SEPTEMBER 4, 1996

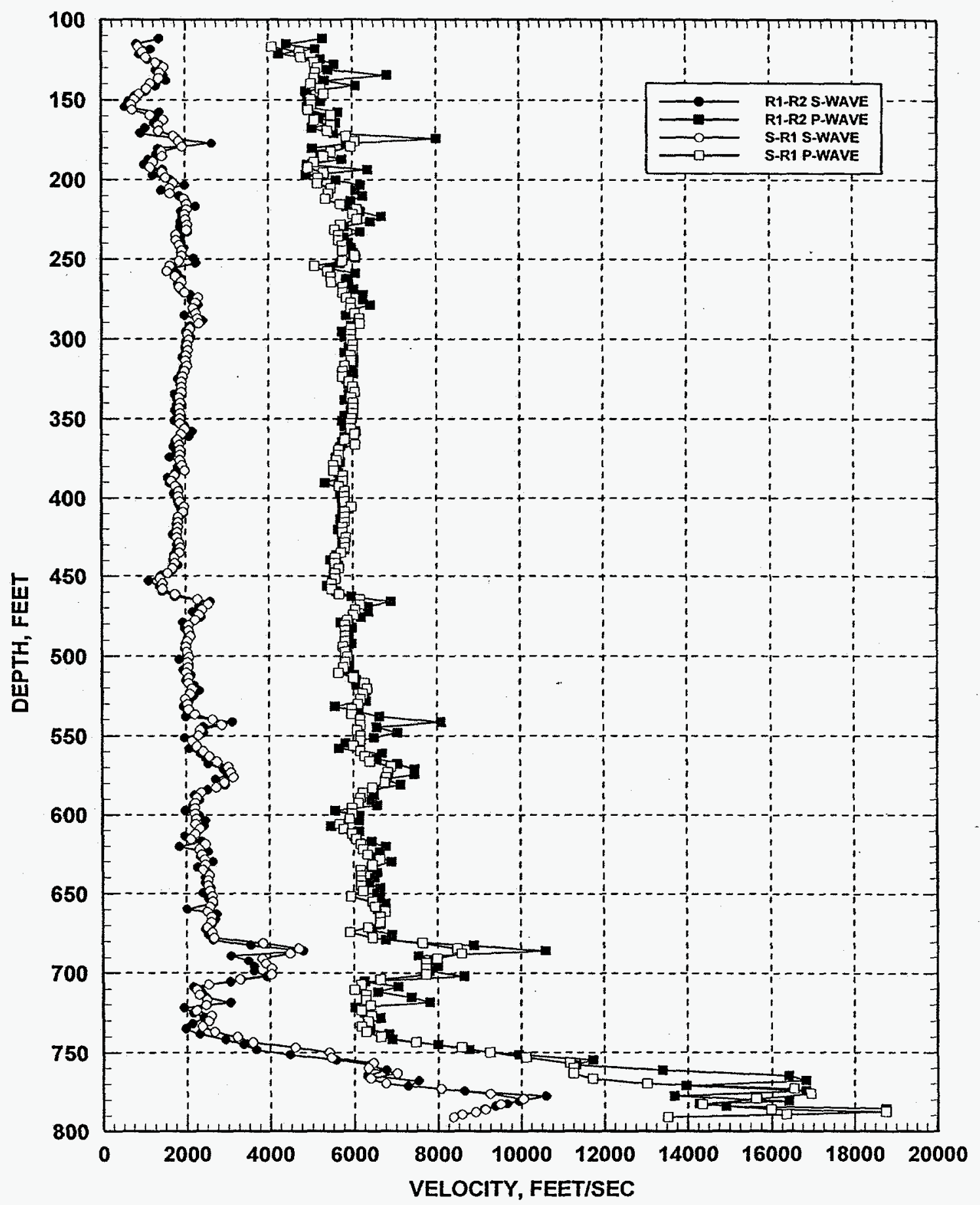

Figure A-1. GCB-1 R1 - R2 high resolution analysis and S-R1 quality assurance analysis $\mathrm{P}$ - and $\mathrm{S}_{\mathrm{H}}$-wave data 


\section{SRS BOREHOLE GCB-1 OYO SUSPENSION LOGGING}

S-WAVE VELOCITIES, DATA COLLECTED SEPTEMBER 4, 1996

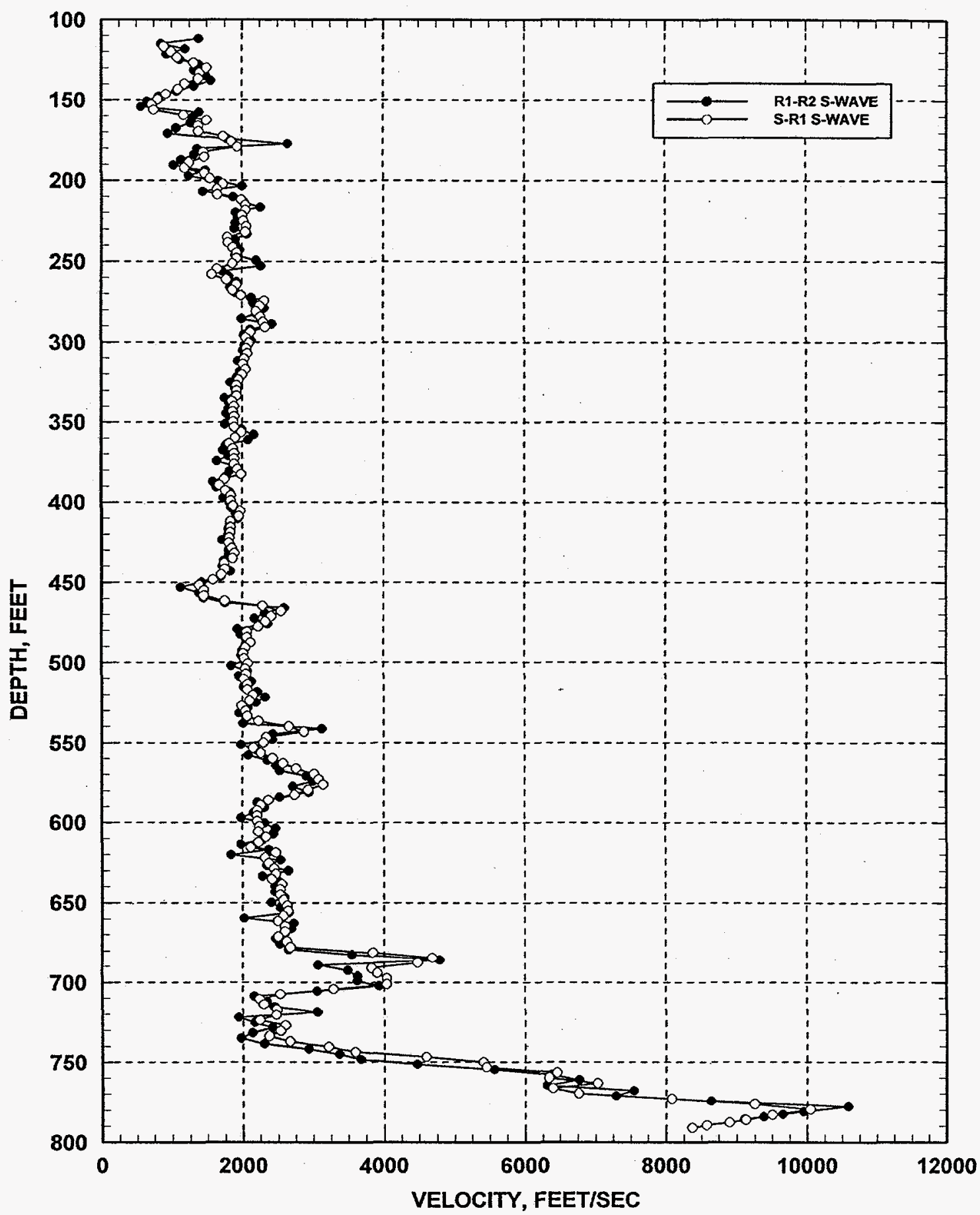

Figure $A-2$. GCB-1 R1 - R2 high resolution analysis and S-R1 quality assurance $\mathrm{S}_{\mathrm{H}}$-wave data 


\begin{tabular}{|c|c|c|}
\hline $\begin{array}{c}\text { Depth } \\
\text { (feet) }\end{array}$ & $\begin{array}{c}\text { S-R1 } \\
\text { Vs (ftsec) }\end{array}$ & $\begin{array}{c}\text { S-R } \text { (ftsec) } \\
\text { V f se }\end{array}$ \\
\hline 116.7 & 892 & 4087 \\
120.0 & 999 & 4750 \\
123.3 & 1082 & 4782 \\
126.5 & 1312 & 5094 \\
129.8 & 1496 & 5169 \\
133.1 & 1396 & 5113 \\
136.4 & 1378 & 5094 \\
139.7 & 1184 & 5021 \\
143.0 & 1092 & 5246 \\
146.2 & 920 & 5326 \\
149.5 & 804 & 5021 \\
152.8 & 725 & 5021 \\
156.1 & 748 & 4951 \\
159.4 & 1172 & 5492 \\
162.6 & 1496 & 5094 \\
165.9 & 1378 & 5492 \\
169.2 & 1384 & 5408 \\
172.5 & 1732 & 5858 \\
175.8 & 1850 & 6060 \\
179.0 & 1931 & 5958 \\
182.3 & 1452 & 5492 \\
185.3 & 1465 & 5326 \\
188.9 & 1251 & 5094 \\
192.2 & 1184 & 4951 \\
195.4 & 1465 & 5326 \\
198.7 & 1535 & 5188 \\
202.0 & 1723 & 5169 \\
205.3 & 1643 & 5492 \\
208.6 & 1644 & 5429 \\
211.8 & 1986 & 5366 \\
215.1 & 2044 & 5715 \\
218.4 & 2044 & 6113 \\
221.7 & 1997 & 6009 \\
225.0 & 2014 & 6113 \\
228.3 & 2056 & 5715 \\
231.5 & 2044 & 5579 \\
234.8 & 1784 & 5669 \\
238.1 & 1793 & 5669 \\
241.4 & 1860 & 5762 \\
244.7 & 1921 & 5762 \\
247.9 & 1921 & 6060 \\
251.2 & 1860 & 5762 \\
254.5 & 1643 & 5094 \\
257.8 & 1569 & 5408 \\
261.1 & 1775 & 5492 \\
264.3 & 1921 & 5492 \\
267.6 & 1860 & 5762 \\
270.9 & 1986 & 5762 \\
274.2 & 2313 & 5858 \\
277.5 & 2253 & 5958 \\
\hline & 2197 & 5958 \\
280.7 & 2260 & 6060 \\
\hline & & 6167 \\
\hline
\end{tabular}

\begin{tabular}{|c|c|c|}
\hline Depth & S-R1 & S-R1 \\
(feet) & Vs (ftsec) & Vp (ftsec) \\
\hline 290.6 & 2328 & 6167 \\
293.9 & 2117 & 5958 \\
297.1 & 2056 & 5958 \\
300.4 & 2092 & 5958 \\
303.7 & 2056 & 6009 \\
307.0 & 2068 & 6009 \\
310.3 & 2026 & 5958 \\
313.6 & 2009 & 5958 \\
316.8 & 2044 & 5810 \\
320.1 & 1997 & 5762 \\
323.4 & 1947 & 5762 \\
326.7 & 1921 & 5908 \\
330.0 & 1921 & 6009 \\
333.2 & 1921 & 6060 \\
336.5 & 1855 & 5958 \\
339.8 & 1880 & 6009 \\
343.1 & 1865 & 6009 \\
346.4 & 1885 & 6009 \\
349.6 & 1875 & 5983 \\
352.9 & 1885 & 5958 \\
356.2 & 1986 & 5958 \\
359.5 & 1897 & 6034 \\
362.8 & 1817 & 5810 \\
366.0 & 1865 & 6060 \\
369.3 & 1885 & 5669 \\
372.6 & 1885 & 5669 \\
375.9 & 1885 & 5624 \\
379.2 & 1931 & 5535 \\
382.5 & 1986 & 5535 \\
385.7 & 1744 & 5762 \\
389.0 & 1678 & 5762 \\
392.3 & 1762 & 5669 \\
395.6 & 1840 & 5810 \\
398.9 & 1840 & 5810 \\
402.1 & 1875 & 5810 \\
405.4 & 1969 & 5958 \\
408.7 & 1947 & 5810 \\
412.0 & 1826 & 5810 \\
415.3 & 1831 & 5810 \\
418.5 & 1826 & 5762 \\
421.8 & 1817 & 5762 \\
425.1 & 1805 & 5834 \\
428.4 & 1850 & 5810 \\
431.7 & 1885 & 5810 \\
434.9 & 1862 & 5715 \\
438.2 & 1740 & 5579 \\
441.5 & 1749 & 5579 \\
444.8 & 1698 & 5669 \\
448.1 & 1583 & 5579 \\
451.3 & 1389 & 5579 \\
454.6 & 1452 & 5492 \\
457.9 & 1452 & 5492 \\
461.2 & 1749 & 5669 \\
\hline & & \\
\hline
\end{tabular}

\begin{tabular}{|c|c|c|}
\hline $\begin{array}{l}\text { Depth } \\
\text { (feet) }\end{array}$ & $\begin{array}{c}\text { S-R1 } \\
\text { Vs }(\mathrm{ft} / \mathrm{sec})\end{array}$ & $\begin{array}{c}\text { S-R1 } \\
\mathrm{Vp} \text { (ft/sec) }\end{array}$ \\
\hline 464.5 & 2282 & 6167 \\
\hline 467.8 & 2547 & 6167 \\
\hline 471.0 & 2408 & 6060 \\
\hline 474.3 & 2328 & 6009 \\
\hline 477.6 & 2225 & 5858 \\
\hline 480.9 & 2068 & 5810 \\
\hline 484.2 & 2068 & 5810 \\
\hline 487.4 & 2117 & 5810 \\
\hline 490.7 & 2044 & 5810 \\
\hline 494.0 & 2014 & 5762 \\
\hline 497.3 & 2026 & 5810 \\
\hline 500.6 & 2080 & 5858 \\
\hline 503.8 & 2044 & 5810 \\
\hline 507.1 & 2056 & 5762 \\
\hline 510.4 & 2020 & 5647 \\
\hline 513.7 & 2068 & 6009 \\
\hline 517.0 & 2068 & 6277 \\
\hline 520.2 & 2143 & 6333 \\
\hline 523.5 & 2099 & 6221 \\
\hline 526.8 & 1986 & 6167 \\
\hline 530.1 & 2044 & 6113 \\
\hline 533.4 & 2068 & 5958 \\
\hline 536.7 & 2225 & 5958 \\
\hline 539.9 & 2653 & 6167 \\
\hline 543.2 & 2869 & 6167 \\
\hline 546.5 & 2336 & 6087 \\
\hline 549.8 & 2297 & 6167 \\
\hline 553.1 & 2150 & 6167 \\
\hline 556.3 & 2260 & 6009 \\
\hline 559.6 & 2424 & 6167 \\
\hline 562.9 & 2575 & 6277 \\
\hline 566.2 & 2757 & 6391 \\
\hline 569.5 & 3017 & 6892 \\
\hline 572.7 & 3083 & 6825 \\
\hline 576.0 & 3145 & 6792 \\
\hline 579.3 & 2929 & 6760 \\
\hline 582.6 & 2735 & 6450 \\
\hline 585.9 & 2367 & 6221 \\
\hline 589.1 & 2260 & 6167 \\
\hline 592.4 & 2211 & 6113 \\
\hline 595.7 & 2211 & 5958 \\
\hline 599.0 & 2211 & 5958 \\
\hline 602.3 & 2239 & 5908 \\
\hline 605.5 & 2225 & 5669 \\
\hline 608.8 & 2336 & 5762 \\
\hline 612.1 & 2225 & 5958 \\
\hline 615.4 & 2111 & 6060 \\
\hline 618.7 & 2467 & 6167 \\
\hline 622.0 & 2313 & 6221 \\
\hline 625.2 & 2367 & 6333 \\
\hline 628.5 & 2441 & 6632 \\
\hline 631.8 & 2467 & 6450 \\
\hline 635.1 & 2408 & 6167 \\
\hline
\end{tabular}

Table A-1. GCB-1 S - R1 quality assurance analysis $P$ - and $S_{H}$-wave data 


\begin{tabular}{|c|c|c|}
\hline $\begin{array}{l}\text { Depth } \\
\text { (feet) }\end{array}$ & $\begin{array}{c}\text { S-R1 } \\
\text { Vs (ft/sec) }\end{array}$ & $\begin{array}{c}\text { S-R1 } \\
\mathrm{Vp}(\mathrm{ft} / \mathrm{sec})\end{array}$ \\
\hline $\begin{array}{l}638.4 \\
641.6\end{array}$ & $\begin{array}{l}2556 \\
2529\end{array}$ & $\begin{array}{l}6167 \\
6167\end{array}$ \\
\hline 644.9 & 2529 & 6167 \\
\hline 648.2 & 2575 & 6221 \\
\hline 651.5 & 2628 & 5932 \\
\hline 654.8 & 2643 & 6450 \\
\hline 658.0 & 2575 & 6509 \\
\hline 661.3 & 2497 & 6760 \\
\hline 664.6 & 2594 & 6632 \\
\hline 667.9 & 2594 & 6632 \\
\hline 671.2 & 2502 & 6333 \\
\hline 674.4 & 2623 & 5908 \\
\hline 677.7 & 2673 & 6450 \\
\hline 681.0 & 3842 & 7641 \\
\hline 684.3 & 4687 & 8470 \\
\hline 687.6 & 4478 & 8573 \\
\hline 690.9 & 3821 & 7989 \\
\hline 694.1 & 3906 & 7725 \\
\hline 697.4 & 4040 & 7725 \\
\hline 701.0 & 4040 & 7725 \\
\hline 704.0 & 3285 & 6632 \\
\hline 707.3 & 2529 & 6167 \\
\hline 710.5 & 2239 & 6009 \\
\hline 713.8 & 2297 & 6277 \\
\hline 717.1 & 2484 & 6277 \\
\hline 720.4 & 2467 & 6391 \\
\hline 723.7 & 2239 & 6167 \\
\hline 726.9 & 2594 & 6420 \\
\hline 730.2 & 2529 & 6333 \\
\hline 733.5 & 2367 & 6167 \\
\hline 736.8 & 2663 & 6277 \\
\hline 740.1 & 3210 & 6632 \\
\hline 743.3 & 3587 & 7479 \\
\hline 746.6 & 4595 & 8573 \\
\hline 749.9 & 5408 & 9250 \\
\hline 753.2 & 5450 & 10115 \\
\hline 756.5 & 6450 & 11159 \\
\hline 759.7 & 6333 & 11248 \\
\hline 763.0 & 7030 & 11248 \\
\hline 766.3 & 6391 & 11717 \\
\hline 769.6 & 6760 & 13019 \\
\hline 772.9 & 8080 & 16541 \\
\hline 776.2 & 9250 & 16940 \\
\hline 779.4 & 10043 & 15622 \\
\hline 782.7 & 9500 & 14347 \\
\hline 786.0 & 9130 & 15977 \\
\hline 787.6 & 8899 & 18747 \\
\hline 789.3 & 8573 & 16349 \\
\hline 790.9 & 8369 & 13519 \\
\hline
\end{tabular}

Table A-1 continued. GCB-1 S - R1 quality assurance analysis $\mathrm{P}$ - and $\mathrm{S}_{\mathrm{H}}$-wave data 


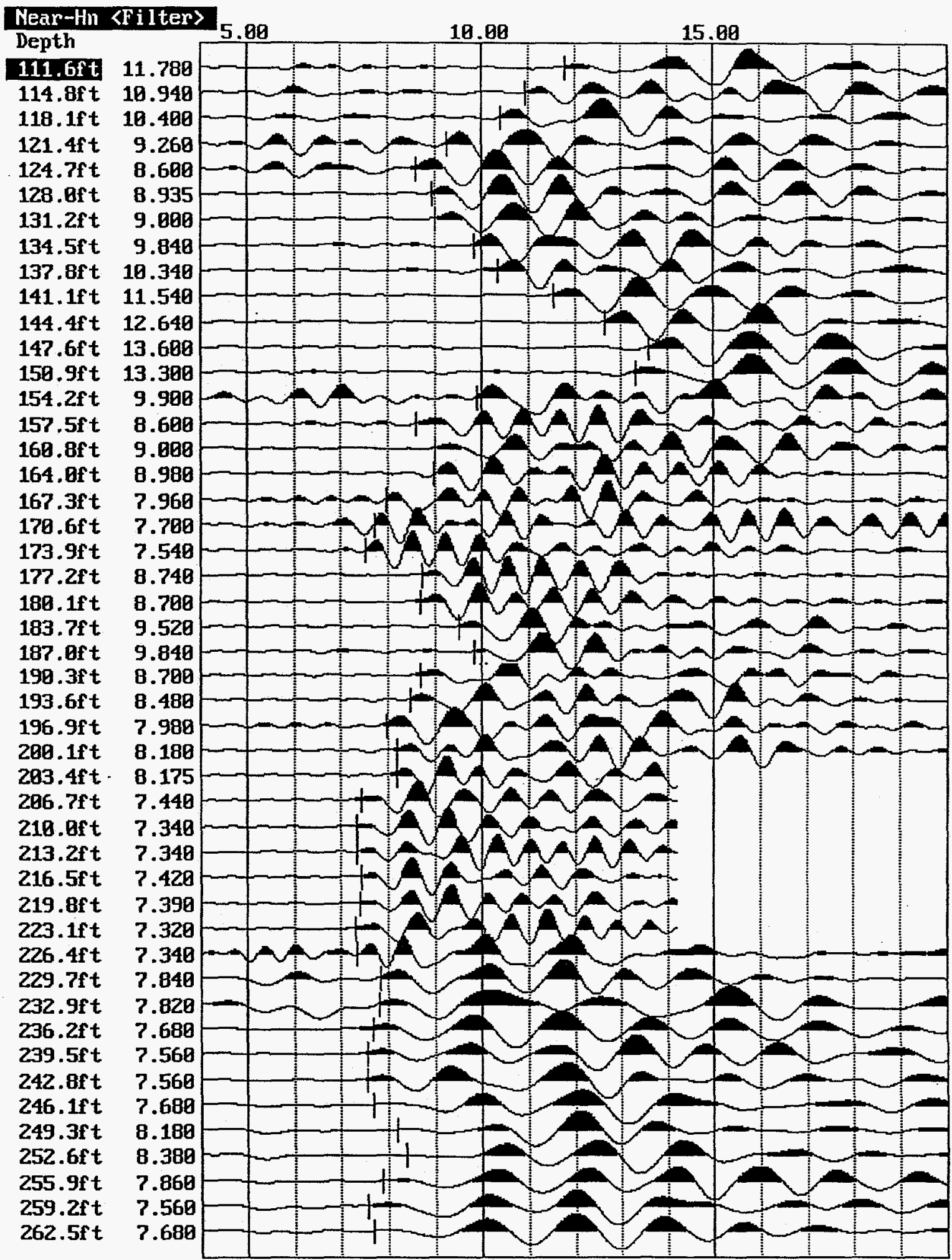

Figure B-1. GCB-1 Suspension $\mathrm{S}_{\mathrm{H}}$-wave $\mathrm{R} 1$ records, "Normal" direction, filtered with S - R1 first arrival picks in milliseconds 


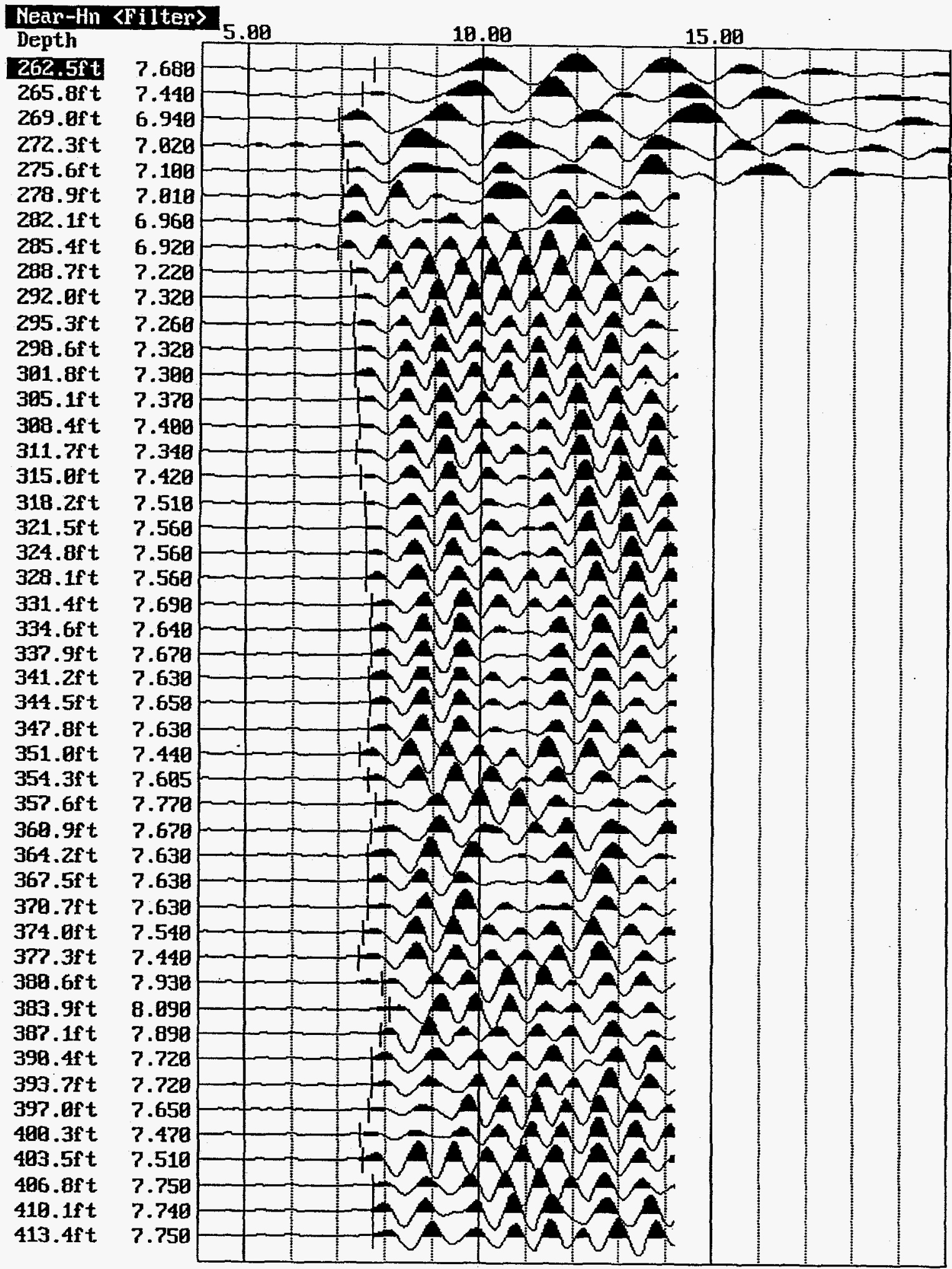

Figure B-1 continued. GCB-1 Suspension $S_{H}$-wave $R 1$ records, "Normal" direction, filtered with S - R1 first arrival picks in milliseconds 


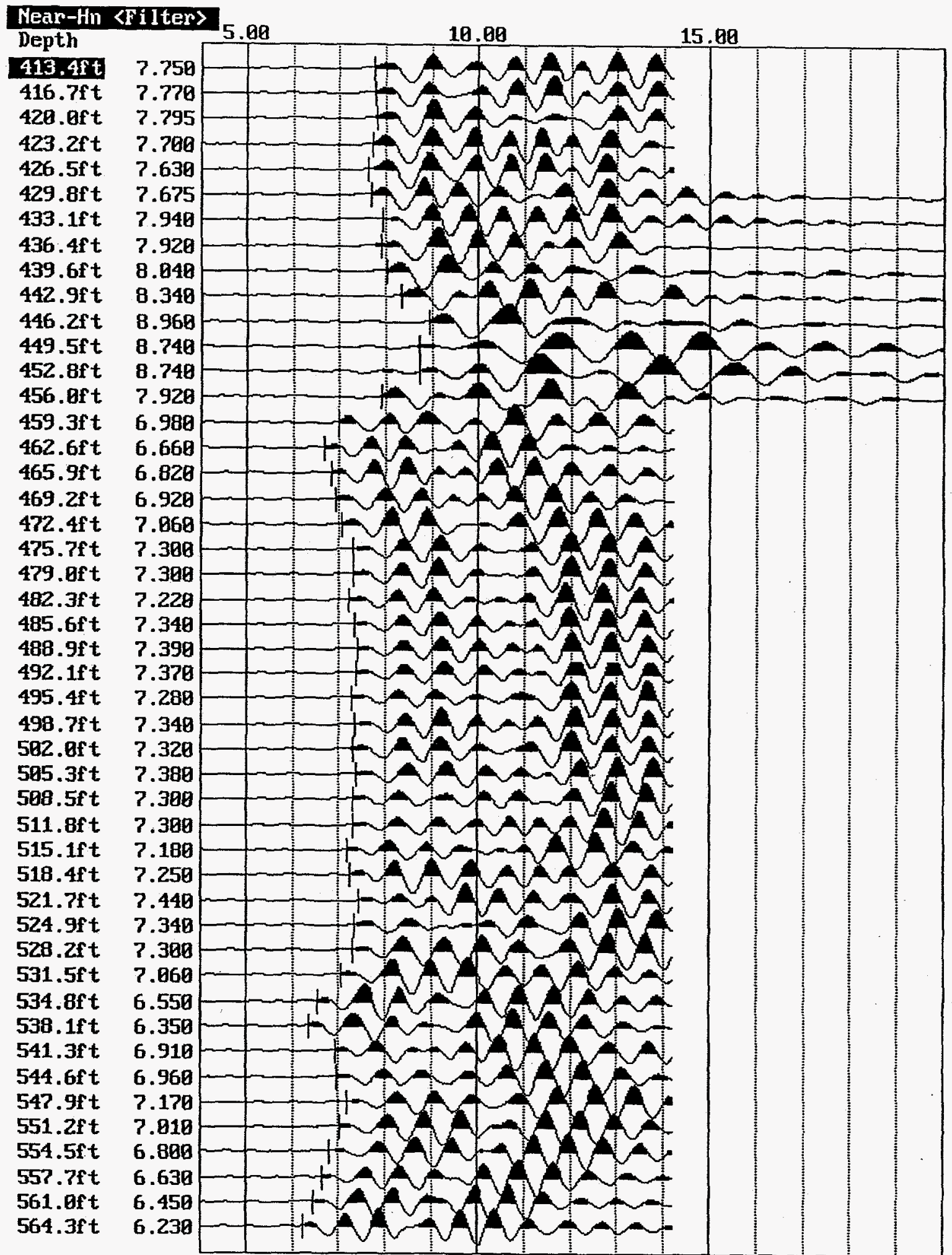

Figure B-1 continued. GCB-1 Suspension $\mathrm{S}_{\mathrm{H}}$-wave $\mathrm{R} 1$ records, "Normal" direction, filtered with S - R1 first arrival picks in milliseconds 


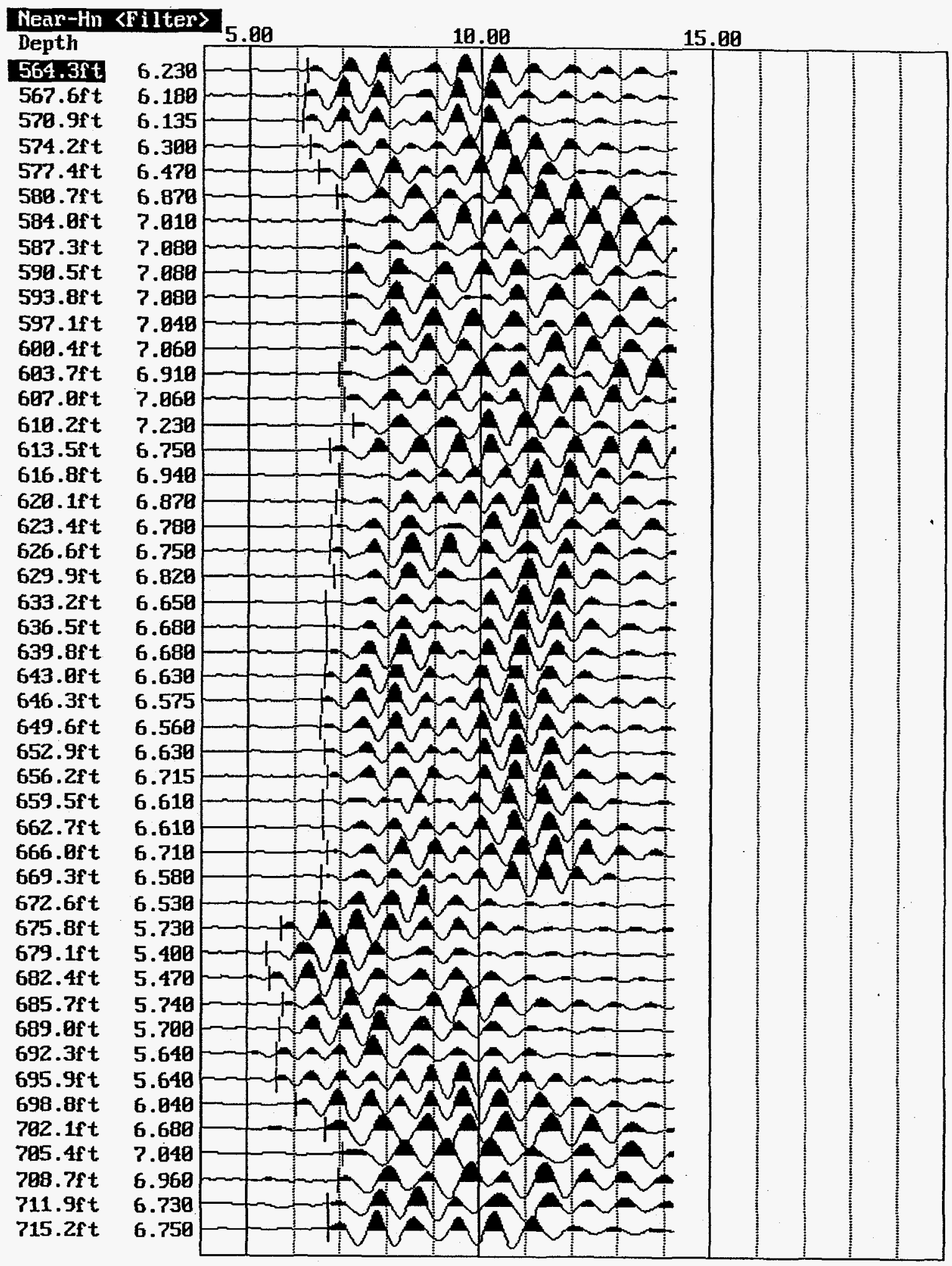

Figure B-1 continued. GCB-1 Suspension $\mathrm{S}_{\mathrm{H}}$-wave $\mathrm{R} 1$ records, "Normal" direction, filtered with S - R1 first arrival picks in milliseconds .

B-5 
718.5ft

721.8ft

725.1ft

728.3ft

731.6ft

$734.9 f t$

738.2ft

$741.5 f t$

P44.8ft

748. Bft

751.3ft

754.6ft

$757.9 f t$

$761.2 \mathrm{ft}$

$764.4 f t$

76?.7ft

771. aft

774.3f $t$

777.6ft

780. Bft

$782.5 \mathrm{ft}$

784. Ift

785.8ft

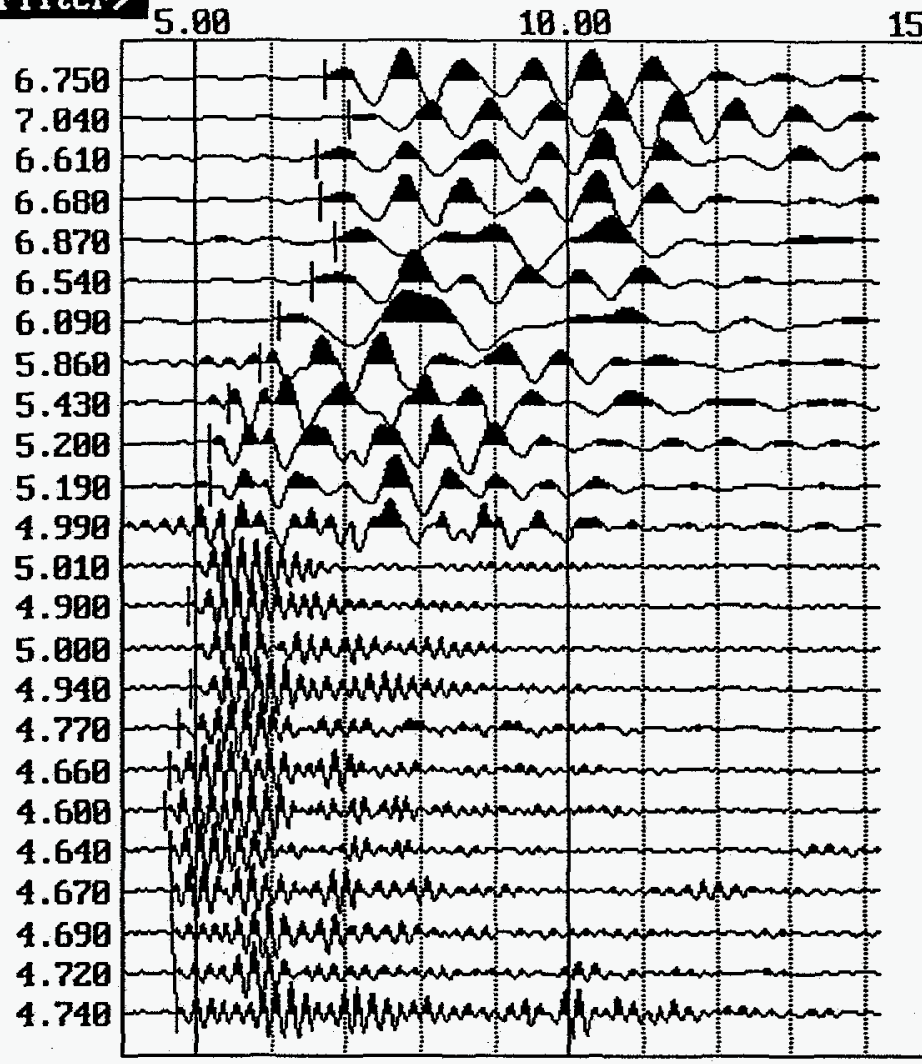

15.00

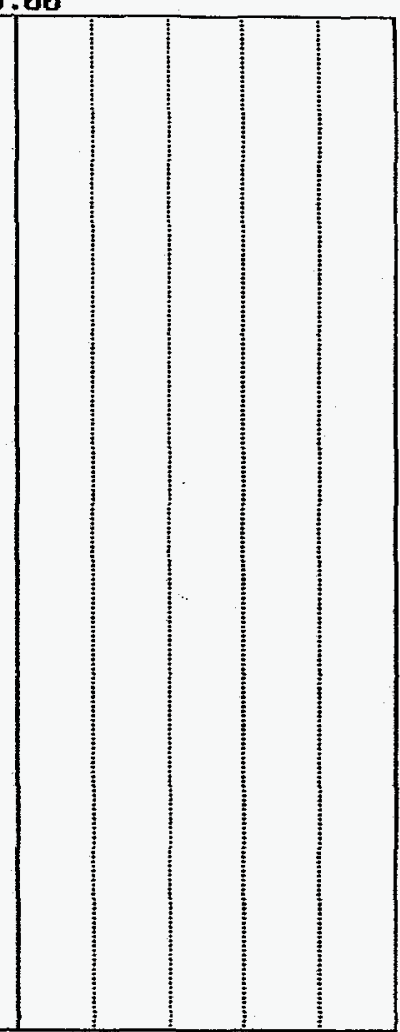

Figure B-1 continued. GCB-1 Suspension $S_{H}$-wave R.1 records, "Normal" direction, filtered with S - R1 first arrival picks in milliseconds 
APPENDIX C

\section{SUSPENSION P-WAVE R1 WAVEFORM} RECORDS WITH S - R1 FIRST ARRIVAL PICKS 


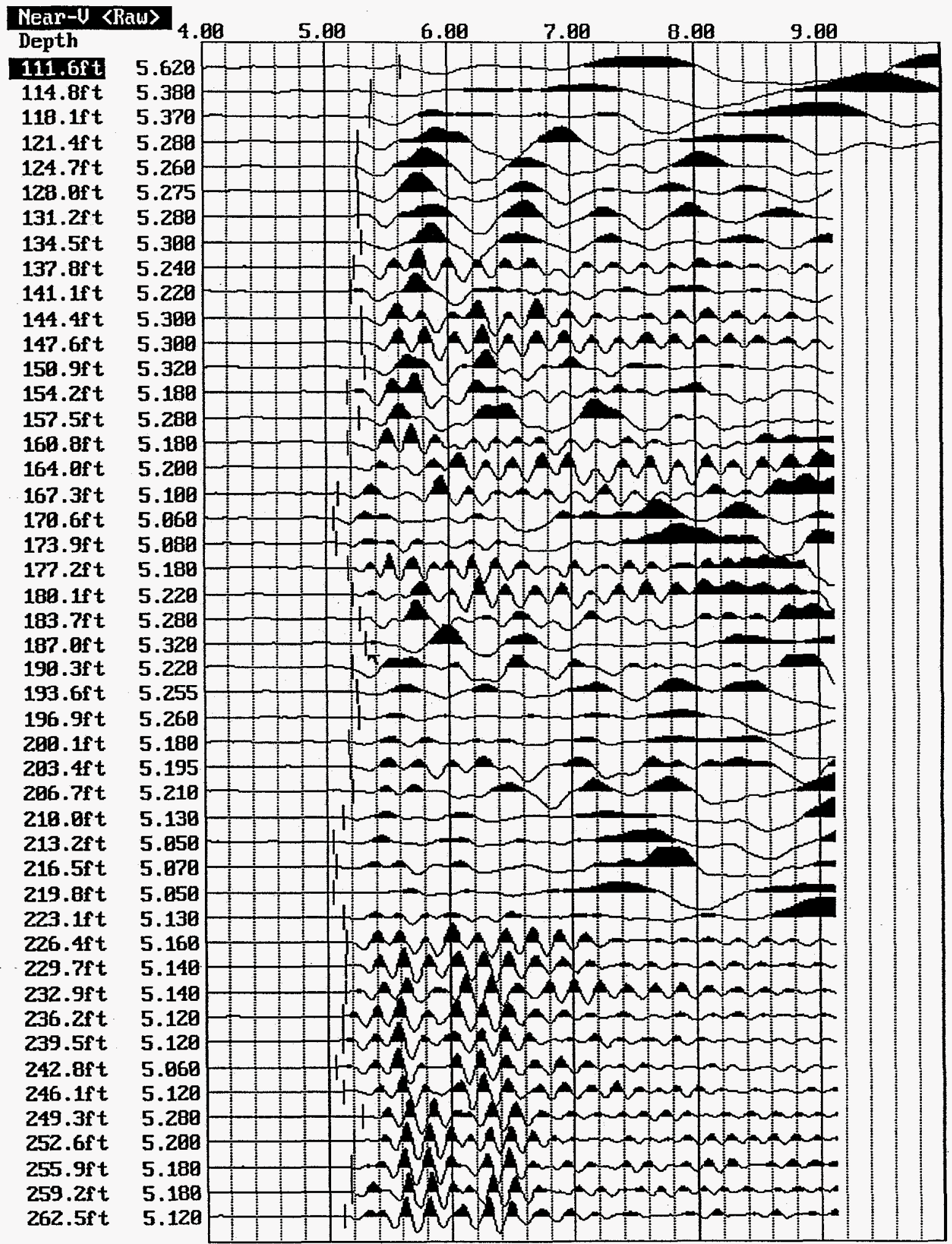

Figure C-1. GCB-1 Suspension P-wave R1 records, Vertical, unfiltered with S - R1 first arrival picks in milliseconds 


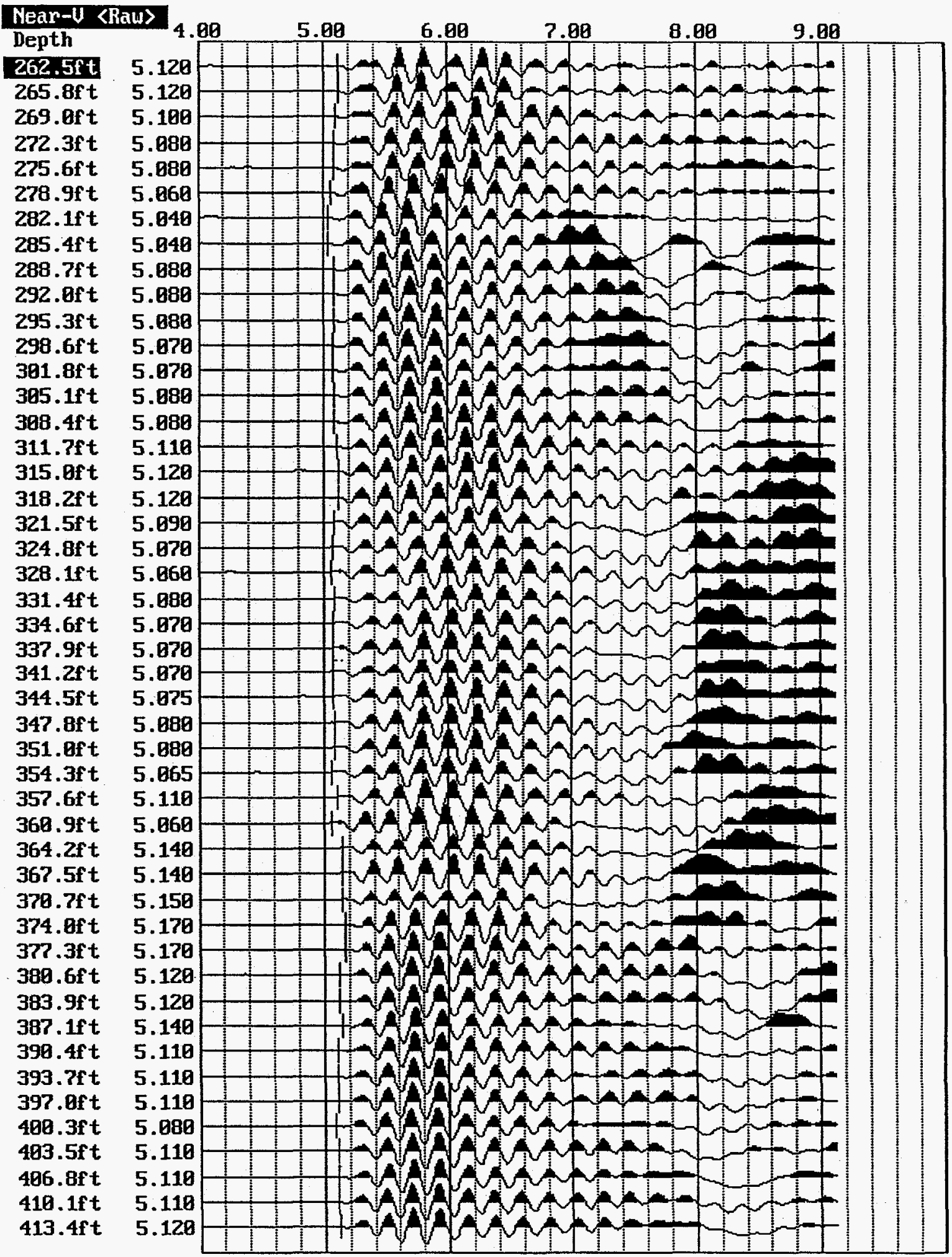

Figure C-1 continued. GCB-1 Suspension P-wave R1 records, Vertical, unfiltered with $S-R 1$ first arrival picks in milliseconds 


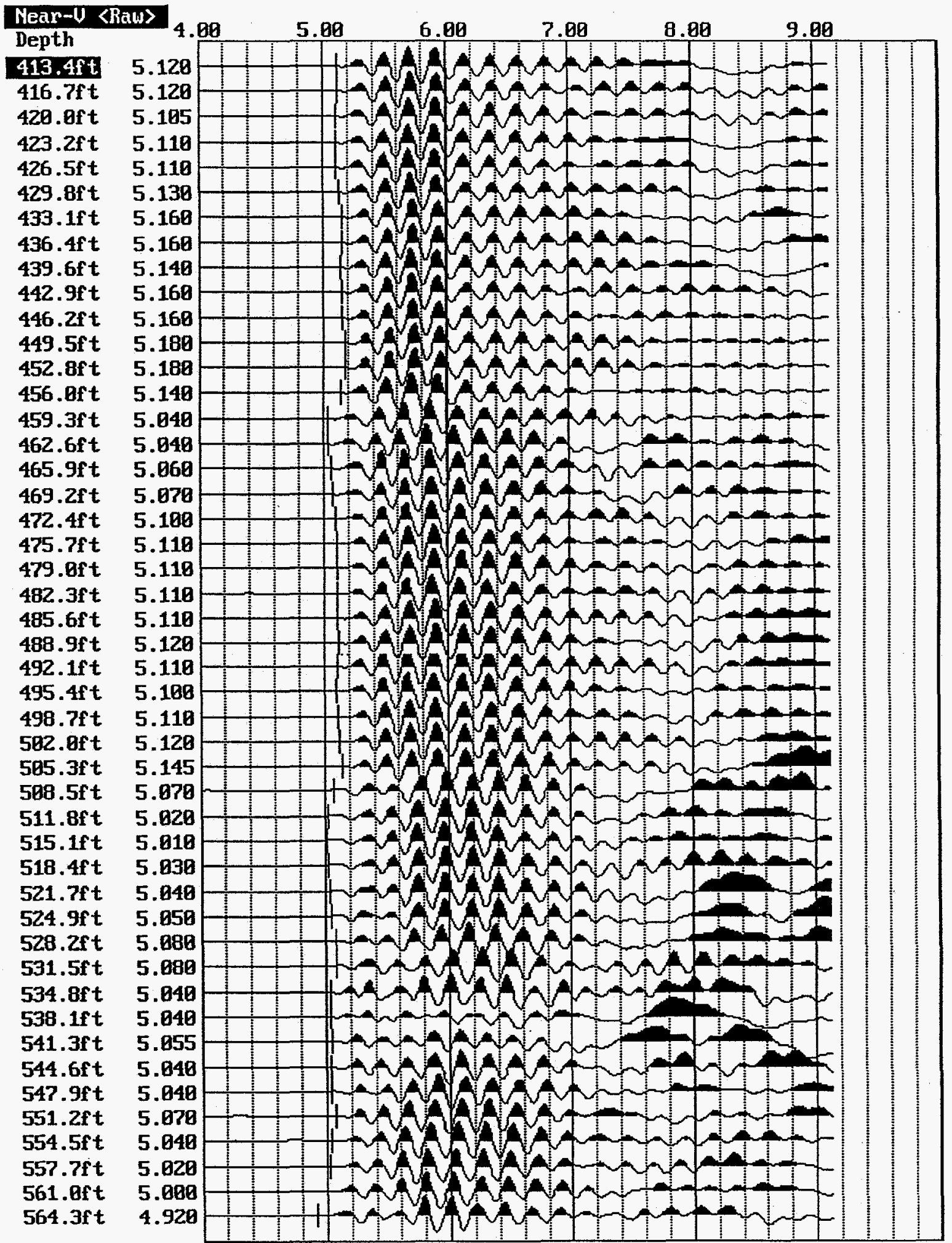

Figure C-1 continued. GCB-1 Suspension P-wave R1 records, Vertical, unfiltered with $S$ - R1 first arrival picks in milliseconds 


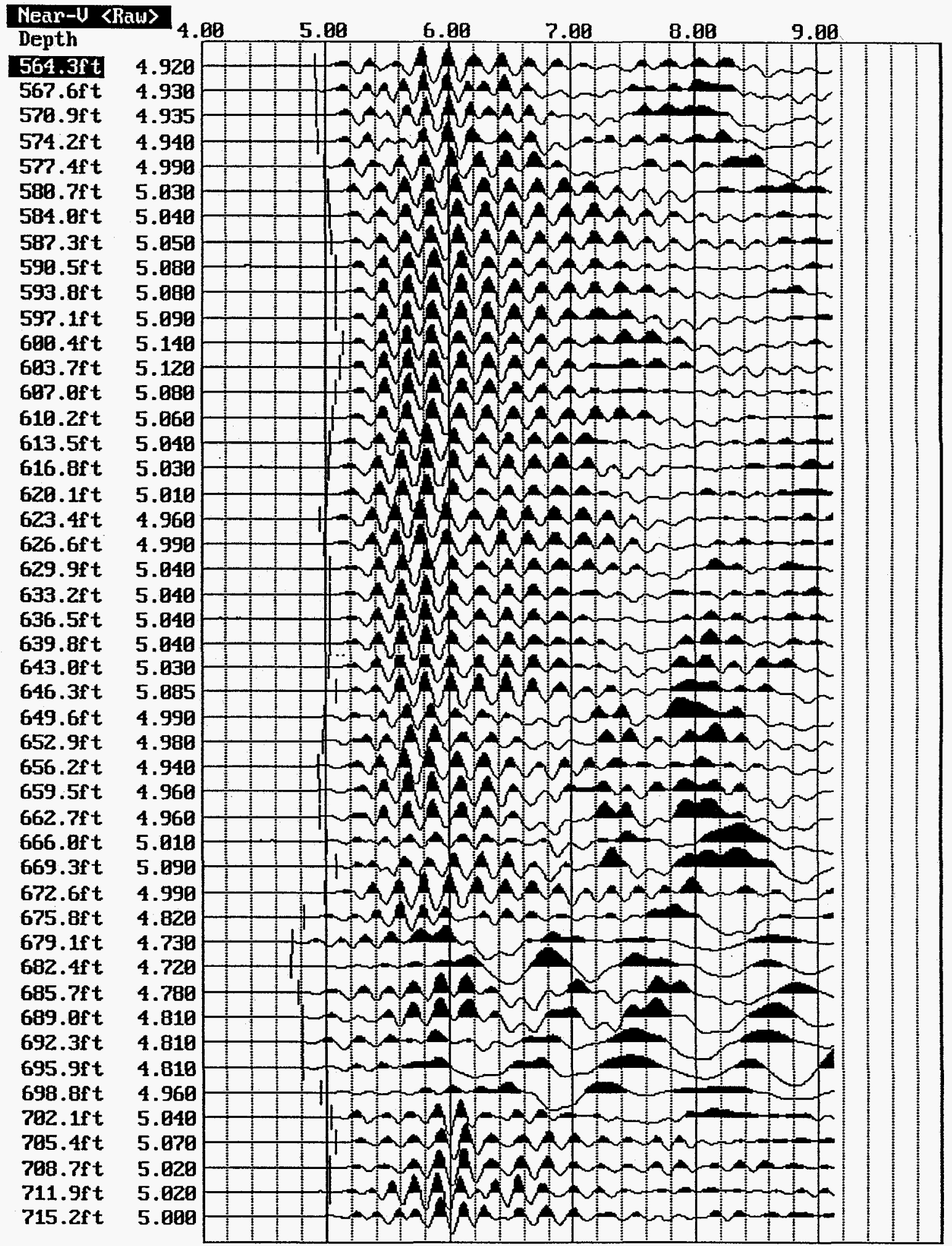

Figure $\mathrm{C}-1$ continued. GCB-1 Suspension P-wave R1 records, Vertical, unfiltered with $S$ - R1 first arrival picks in milliseconds 


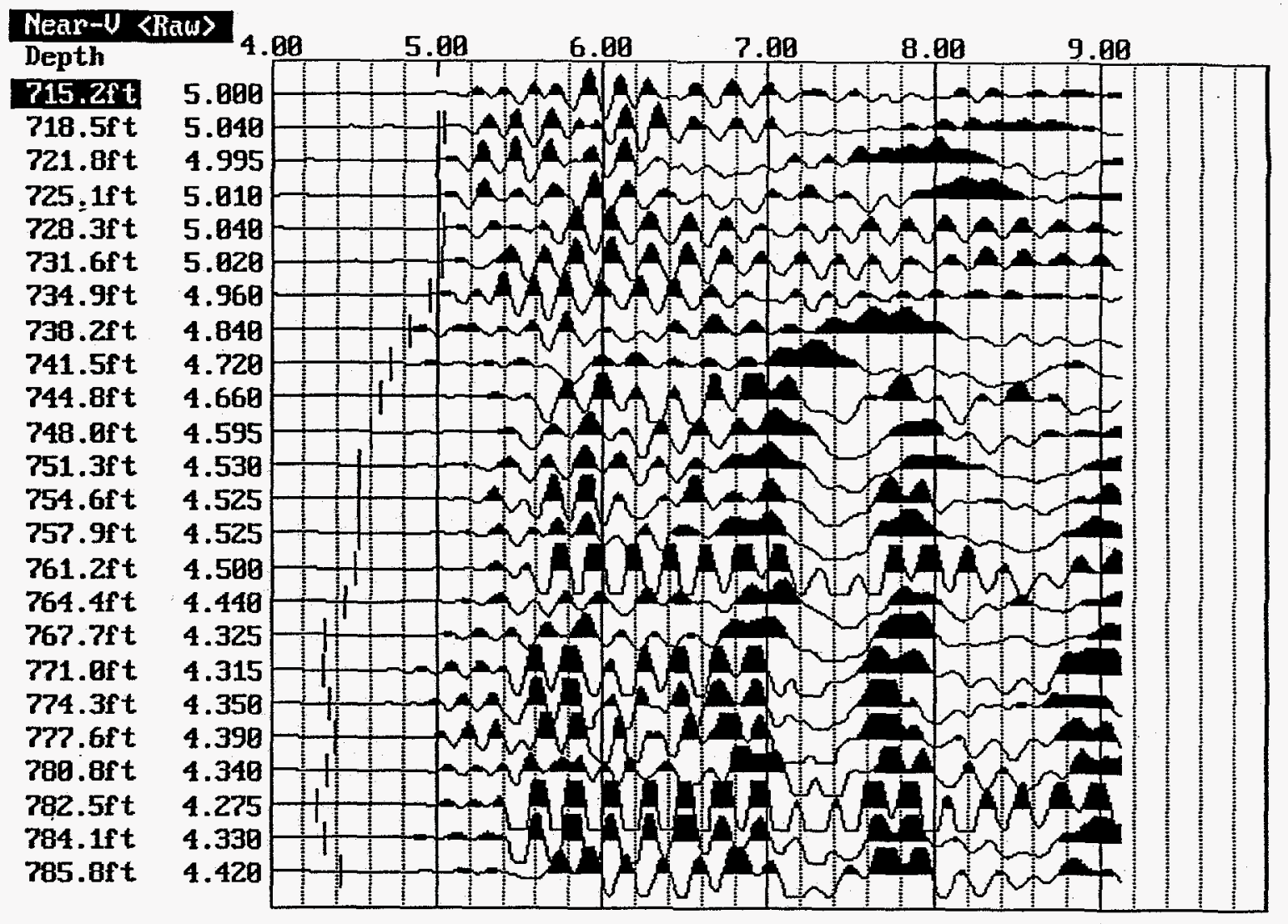

Figure $\mathrm{C}-1$ continued. GCB-1 Suspension P-wave R1 records, Vertical, unfiltered with $S$ - R1 first arrival picks in milliseconds 


\title{
SUSPENSION VELOCITY MEASUREMENTS
}

\author{
AT THE \\ SAVANNAH RIVER SITE \\ BOREHOLE GCB-2
}

July 31,1996 


\title{
SUSPENSION VELOCITY MEASUREMENTS
}

\author{
AT THE \\ SAVANNAH RIVER SITE \\ BOREHOLE GCB-2
}

\author{
Prepared for \\ University of South Carolina \\ Earth Sciences and Resources Institute \\ Columbia, South Carolina 29208 \\ (803) 331-6003 \\ Prepared by \\ Agbabian Associates, Inc.: \\ 1111 South Arroyo Parkway, Suite 470 \\ Pasadena, California 91105 \\ (818) 441-1060 \\ Project 96227
}

July 31, 1996

Report.96227-6611 


\section{TABLE OF CONTENTS}

INTRODUCTION

SCOPE OF WORK

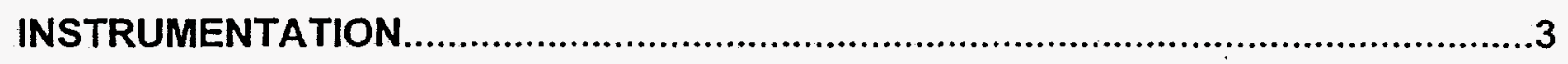

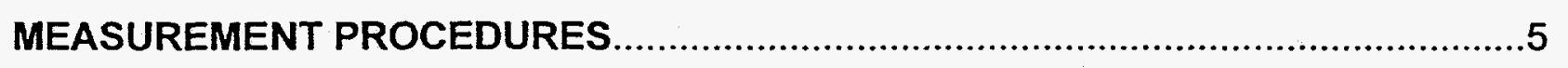

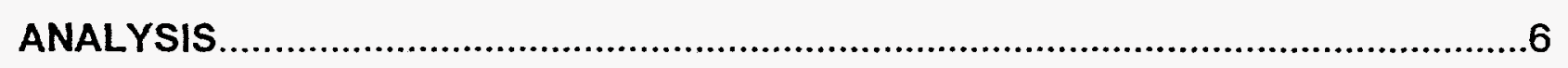

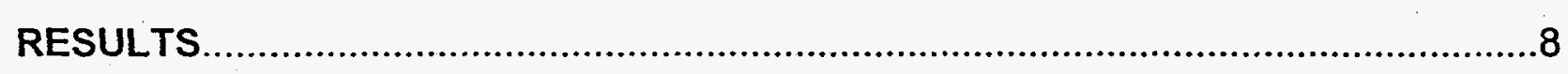

SUMMARY

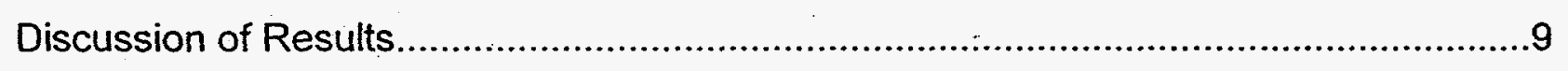

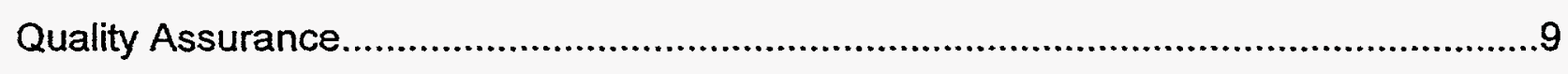

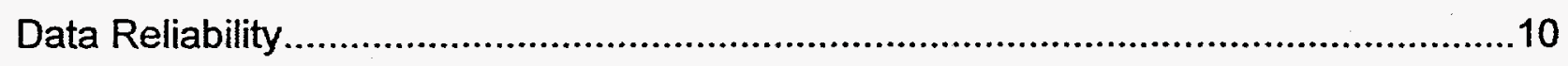

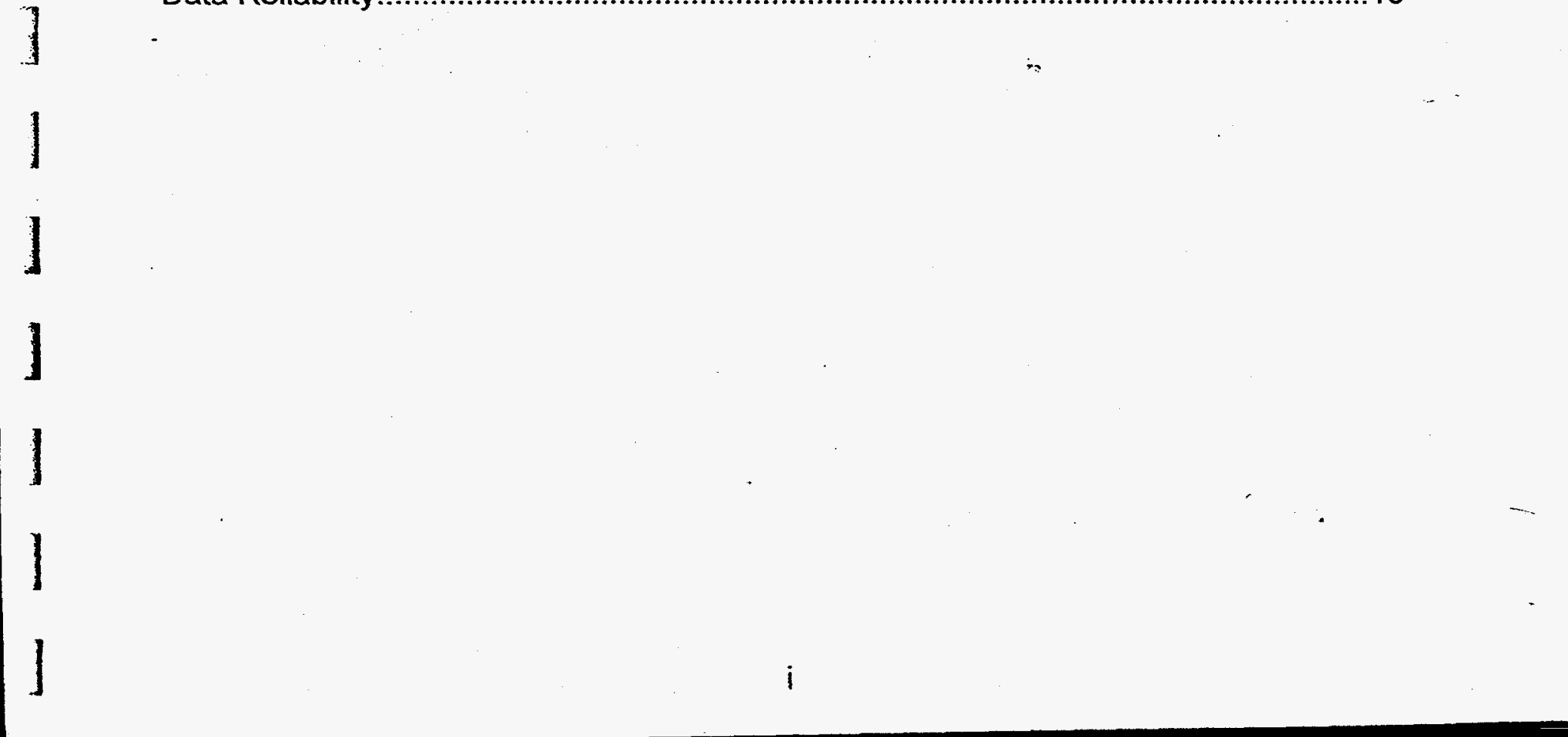




\section{FIGURES}

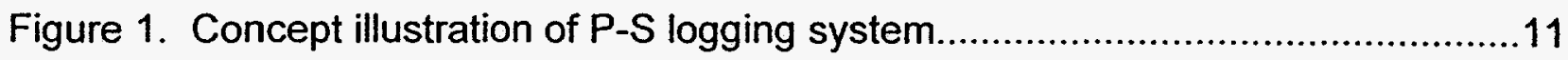

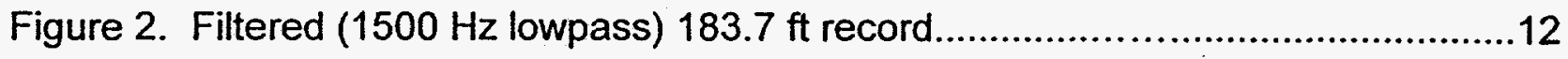

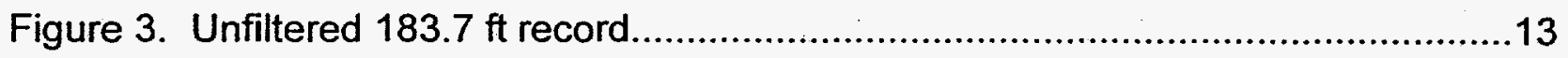

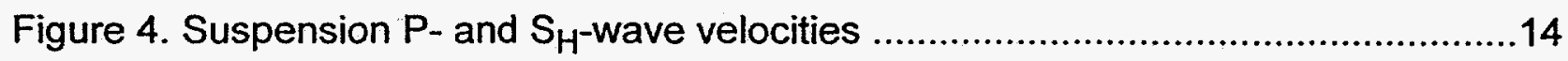

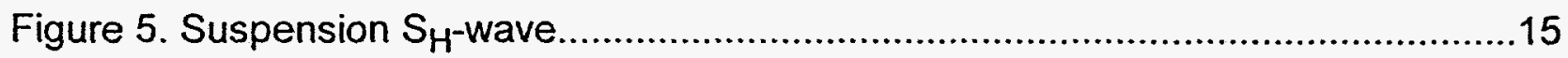

TABLES

Table 1. Suspension R1 - R2 depth, pick times, and velocities................................17

\section{APPENDICES}

APPENDIX A: Suspension velocity measurement quality assurance suspension source to receiver analysis results

APPENDIX B: Suspension $S_{H}$-wave $R 1$ waveform records with S-R1 first arrival picks

APPENDIX C: Suspension P-wave R1 waveform records with S-R1 first arrival picks

APPENDIX D: OYO Model 170 suspension velocity logging system NIST traceable calibration procedure 


\section{INTRODUCTION}

Suspension velocity measurements were performed in borehole GCB-2 at the Savannah River Site, near Aiken, South Carolina. Suspension P-and $\mathrm{S}_{\mathrm{H}}$-velocity measurements were collected to a maximum depth of $668.3 \mathrm{ft}$. Data acquisition was performed on June 30, 1996. The work was conducted by Rob Steller of Agbabian Associates for the University of South Carolina and the Westinghouse Savannah River Company. Mr. Mike Waddell was the technical liaison for the University of South Carolina, with Mr. Doug Wyatt serving as project liaison for Westinghouse.

This report describes the field measurements, data analysis, and results of this work. 


\section{SCOPE OF WORK}

This report presents the results of suspension velocity measurements on June 30, 1996, between depths of 144.4 and $668.3 \mathrm{ft}$ in the nominal 5 inch uncased borehole designated GCB-2, located at the Savannah River Site, near Aiken, South Carolina. The purpose of these measurements was to acquire shear wave velocities and compressional wave velocities as a function of depth which, in turn, will be used to characterize ground response to earthquake motion.

The OYO Model 170 Suspension Logging Recorder and Suspension Logging Probe were used to obtain in-situ horizontal shear and compressional wave velocity measurements at 3.28 or $1.64 \mathrm{ft}$ intervals. The acquired data was analyzed and a profile of velocity versus depth was produced for both compressional and horizontally polarized shear waves.

A detailed reference for the velocity measurement techniques used in this study is:

Guidelines for Determining Design Basis Ground Motions, Report TR-102293, Electric Power Research Institute, Palo Alto, California, November 1993, Sections 7 and 8. 


\section{INSTRUMENTATION}

Suspension soil velocity measurements were performed using the Model 170 Suspension Logging system, manufactured by OYO Corporation. This system directly determines the average velocity of a $3.28 \mathrm{ft}$ high segment of the soil column surrounding the borehole of interest by measuring the elapsed time between arrivals of a wave propagating upward through the soil column. The geophones that detect the wave, and the source that generates the wave, are moved as a unit in the borehole producing relatively constant amplitude signals at all depths.

The suspension system probe consists of a combined reversible polarity solenoid horizontal shear-wave generator $\left(\mathrm{S}_{\mathrm{H}}\right)$ and compressional-wave generator $(\mathrm{P})$, joined to two biaxial geophones by a flexible isolation cylinder, as shown in Figure 1. The separation of the two geophones is $3.28 \mathrm{ft}$, allowing average wave velocity in the region between the geophones to be determined by inversion of the wave travel time between the two geophones. The total length of the probe as used in this survey is approximately $22.0 \mathrm{ft}$; the center point of the geophones is approximately $14.8 \mathrm{ft}$ above the bottom end of the probe. The probe receives control signals from, and sends the amplified geophone signals to, instrumentation on the surface via an armored 7 conductor cable. The cable is wound onto the drum of a winch and is used to support the probe. Cable travel is measured to provide probe depth data.

The entire probe is suspended by the cable and centered in the borehole by nylon "whiskers", therefore, source motion is not coupled directly to the borehole walls; rather, the source motion creates a horizontally propagating impulsive pressure wave in the fluid filling the borehole and surrounding the source. This pressure wave is converted to $\mathrm{P}$ and $\mathrm{S}_{\mathrm{H}^{-}}$waves in the surrounding soil and rock as it impinges upon the borehole wall. These waves propagate through the soil and rock surrounding the borehole, in turn causing a pressure wave to be generated in the fluid surrounding the geophones as the soil waves pass their location.

In operation, a distinct, repeatable pattern of impulses is generated at each depth as follows:

1. The source is fired in one direction producing dominantly horizontal shear with some vertical compression, and the signals from the horizontal geophones situated parallel to the axis of motion of the source are recorded. 
2. The source is fired again in the opposite direction and the horizontal geophone signals are recorded.

3. The source is fired again and the vertical geophone signals are recorded. The repeated source pattern facilitates the picking of the $\mathrm{P}$ and $\mathrm{S}_{\mathrm{H}}$-wave arrivals; reversal of the source changes the polarity of the $\mathrm{S}_{\mathrm{H}}$-wave pattern but not the $\mathrm{P}$-wave pattern.

The data from each geophone during each source activation is recorded as a different channel on the recording system. The Model 170 has six channels (two simultaneous recording channels), each with a 12 bit 1024 sample record. The recorded data is displayed on a CRT display and on paper tape output as six channels with a common time scale. Data is stored on 3.5 inch floppy diskettes for further processing. Up to 8 sampling sequences can be summed to improve the signal to noise ratio of the signals.

Review of the displayed data on the CRT or paper tape allows the operator to set the gains, filters, delay time, pulse length (energy), sample rate, and summing number in order to optimize the quality of the data before recording. Calibration of the Model 170 digital recorder is performed every six months, using a NITS traceable frequency source and counter, as outlined in Appendix D. 


\section{MEASUREMENT PROCEDURES}

The borehole was logged as a $684 \mathrm{ft}$ deep, nominal 5 inch diameter uncased borehole filled with bentonite based drilling mud. The suspension probe was positioned with the mid-point of the receiver spacing at grade level, and mechanical and electronic depth counters were set to zero. The probe was lowered to the bottom of the borehole, then returned to the bottom of the steel conductor casing at $144.4 \mathrm{ft}$, stopping at 3.28 or $1.64 \mathrm{ft}$ intervals to collect data. During this procedure, the deepest measurement was taken with the probe depth reference point located at $668.3 \mathrm{ft}$, placing the tip of the probe within $1 \mathrm{ft}$ of the bottom of the borehole at approximately $683.1 \mathrm{ft}$.

At each measurement depth the measurement sequence of two opposite horizontal records and one vertical record was performed, and the gains were adjusted as required. The data from each depth was printed on paper tape, checked, and recorded on diskette before moving to the next depth.

Upon completion of the measurement run, the probe zero depth indication was verified prior to removal from the borehole. 


\section{DATA ANALYSIS}

The recorded digital records were analyzed to locate the first minima on the vertical axis records, indicating the arrival of P-wave energy. The difference in travel time between receiver 1 and receiver $2(\mathrm{R} 1-\mathrm{R} 2)$ arrivals was used to calculate the P-wave velocity for that $3.28 \mathrm{ft}$ interval. When observable, P-wave arrivals on the horizontal axis records were used to verify the velocities determined from the vertical axis data.

The P-wave velocity calculated from the travel time over the $10.3 \mathrm{ft}$ interval from source to receiver $\mathrm{l}(\mathrm{S}-\mathrm{R} \mathrm{l})$ was calculated and plotted for quality assurance of the velocity derived from the travel time between receivers. In this analysis, the depth values as recorded were increased by $6.8 \mathrm{ft}$ to correspond to the mid-point of the $10.3 \mathrm{ft} \mathrm{S-R1}$ interval. Travel times were obtained by picking the first break of the P-wave signal at the near receiver and subtracting 3.90 milliseconds, the calculated and experimentally verified delay from source trigger pulse (beginning of record) to source impact. This delay corresponds to the duration of acceleration of the solenoid before impact.

The recorded digital records were studied to establish the presence of clear $\mathrm{S}_{\mathrm{H}}$-wave pulses, as indicated by the presence of opposite polarity pulses on each pair of horizontal records. Ideally, the $\mathrm{S}_{\mathrm{H}}$-wave signals from the 'normal' and 'reverse' source pulses are very nearly inverted images of each other. Digital FFT - IFFT lowpass filtering was used to remove the higher frequency Pwave signal from the $\mathrm{S}_{\mathrm{H}^{-}}$-wave signal. Different filter cutoffs were used to separate $\mathrm{P}$ - and $\mathrm{S}_{\mathrm{H}^{-}}$. -waves at different depths, ranging from $1000 \mathrm{~Hz}$ in the slowest zones to $2500 \mathrm{~Hz}$ in the regions of highest velocity.

Generally, the first maxima was picked for the 'normal' signals and the first minima for the 'reverse' signals, although other points on the waveform were used if the first pulse was distorted. The absolute arrival time of the 'normal' and 'reverse' signals may vary by $+/-0.2$ milliseconds, due to differences in the actuation time of the solenoid source caused by constant mechanical bias in the source or by borehole inclination. This variation does not affect the R1-R2 velocity determinations, as the differential time is measured between arrivals of waves created by the 
same source actuation. The final velocity value is the average of the values obtained from the 'normal' and 'reverse' source actuations.

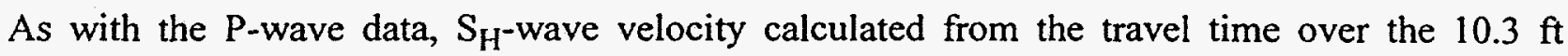
interval from source to first receiver was calculated and plotted for verification of the velocity derived from the travel time between receivers. In this analysis, the depth values were increased by $6.8 \mathrm{ft}$ to correspond to the mid-point of the $10.3 \mathrm{ft} \mathrm{S}-\mathrm{R} 1$ interval. Travel times were obtained by picking the first break of the $\mathrm{S}_{\mathrm{H}}$-wave signal at the near receiver and subtracting 3.90 milliseconds, the calculated and experimentally verified delay from the beginning of the record at the source trigger pulse to source impact.

Figure 2 shows an example of R1 - R2 measurements on the filtered record for a depth of 183.7 $\mathrm{ft}$. In Figure 2, the time difference over the $3.28 \mathrm{ft}$ interval of 1.68 millisecond is equivalent to a $\mathrm{S}_{\mathrm{H}}$-wave velocity of $1953 \mathrm{ft} / \mathrm{sec}$. Whenever possible, time differences were determined from several phase points on the $\mathrm{S}_{\mathrm{H}^{-}}$-waveform records to verify the data obtained from the first arrival of the $\mathrm{S}_{\mathrm{H}}$-wave pulse. Figure 3 displays the same record before filtering with a $1500 \mathrm{~Hz} F F T$ IFFT digital lowpass filter. 


\section{RESULTS}

Suspension R1-R2 P- and $\mathrm{S}_{\mathrm{H}^{-}}$wave velocity are plotted in Figure 4. The $\mathrm{S}_{\mathrm{H}^{-}}$-wave velocity data are presented at an expanded scale in Figure 5. The suspension velocity data presented in Figures 4 and 5 are presented in Table 1 . $\mathrm{P}$ - and $\mathrm{S}_{\mathrm{H}^{-}}$-wave velocity data from $\mathrm{R} 1-\mathrm{R} 2$ analysis and quality assurance analysis of S-R1 data are plotted together in Figures A1 and A2 to aid in visual comparison. It must be noted that R1-R2 data is an average velocity over a $3.28 \mathrm{ft}$ segment of the soil column; $\mathrm{S}-\mathrm{R} 1$ data is an average over $10.3 \mathrm{ft}$, creating a significant smoothing relative to the R1-R2 plots. S-R1 data are presented in tabular format in Table A1. Good correspondence between the shape of the $\mathrm{P}$ - and $\mathrm{S}_{\mathrm{H}}$-wave velocity curves is observed for all data sets. The velocities derived from $S-R 1$ and $R 1-R 2$ data are in close agreement, providing verification of the higher resolution $R 1-R 2$ data.

Depth sequential $\mathrm{S}_{\mathrm{H}^{-}}$and $\mathrm{P}$-wave records with picks from S-R1 analysis used for quality assurance are presented in Appendices B and C. Calibration procedures and records for the measurement system are presented in Appendix D. 


\section{SUMMARY}

\section{Discussion of Results}

Both $\mathrm{P}$ - and $\mathrm{S}_{\mathrm{H}}$-wave velocities were measured using the Suspension Method between depths of $144.4 \mathrm{ft}$ and $668.3 \mathrm{ft}$ in borehole GCB-2, at the Savannah River Site. Data was not collected above $144.4 \mathrm{ft}$ due to the presence of steel conductor casing.

The site was located in a forest, with no apparent sources of ambient vibration nearby.

A significant decrease in $\mathrm{S}_{\mathrm{H}}$-wave velocity is observed between depths of 320 and $340 \mathrm{ft}$, indicating a soft soil layer, possibly clean sands. Another significant change in soil composition is observed between 590 and $620 \mathrm{ft}$, where the rapid increase in $\mathrm{P}$ - and $\mathrm{S}_{\mathrm{H}^{-}}$-wave velocity indicates a rock like layer. Basement rock appears to be encountered at a depth of approximately $660 \mathrm{ft}$.

\section{Quality Assurance}

These velocity measurements at the Savannah River Site, borehole GCB-2, were performed by highly qualified professional staff members of Agbabian Associates, Inc. under the supervision and quality assurance of Dr. Robert L. Nigbor, P.E.. Industry-standard or better methods and levels of care were employed for both measurements and analyses. All work was performed under Agbabian Associates quality assurance procedures, which include:

- Use of NIST-traceable calibrations, where applicable, for field and laboratory instrumentation

- Use of standard field data logs

- Where applicable, use of independent verification of data (for example, comparison of receiver-to-receiver and source-to-receiver velocities for the suspension velocity measurements) 
- Independent review of calculations and results by a registered professional engineer, geologist, or geophysicist.

\section{Data Reliability}

$\mathrm{P}$ - and $\mathrm{S}_{\mathrm{H}^{-}}$wave velocity measurement using the Suspension Method gives average velocities over a $3.28 \mathrm{ft}$ interval of depth. This high resolution results in the scatter of values shown in the graphs. Individual measurements are very reliable; the estimated precision is $+/-5 \%$. Good field procedures and quality assurance add to the high reliability of these data. 


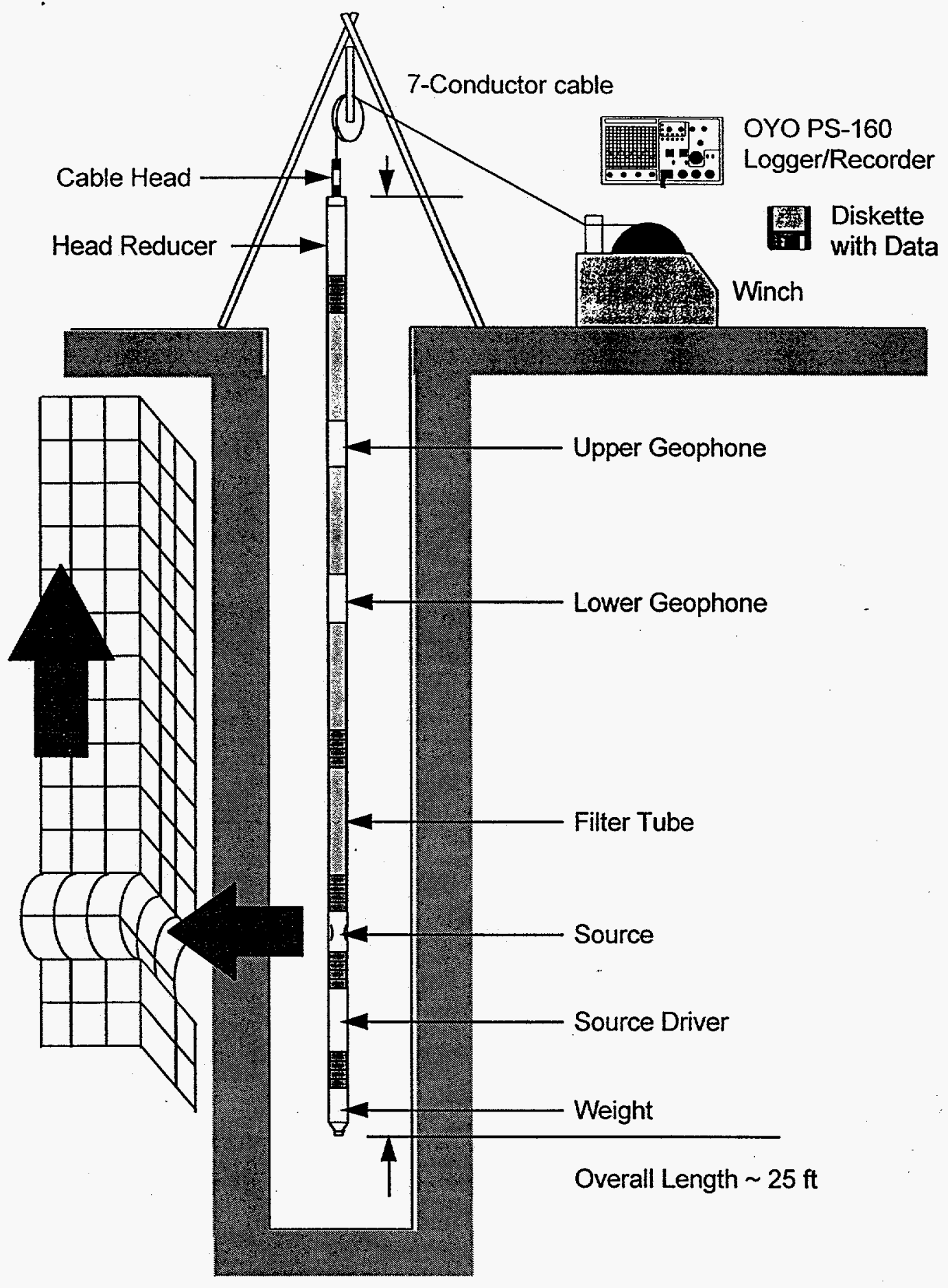

Figure 1. Concept illustration of P-S logging system 


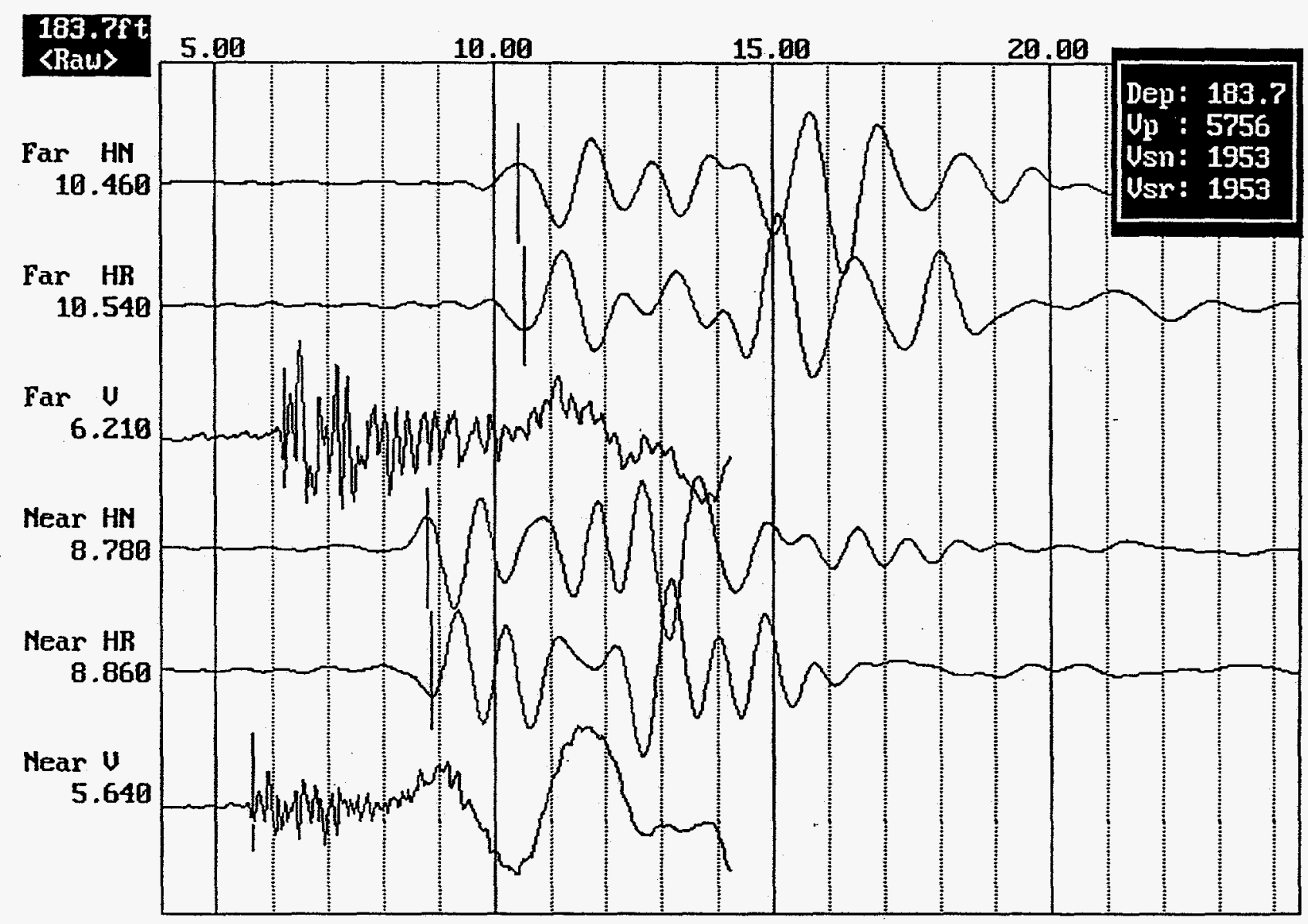

Figure 2. Filtered ( $1500 \mathrm{~Hz}$ lowpass) $183.7 \mathrm{ft}$ record 


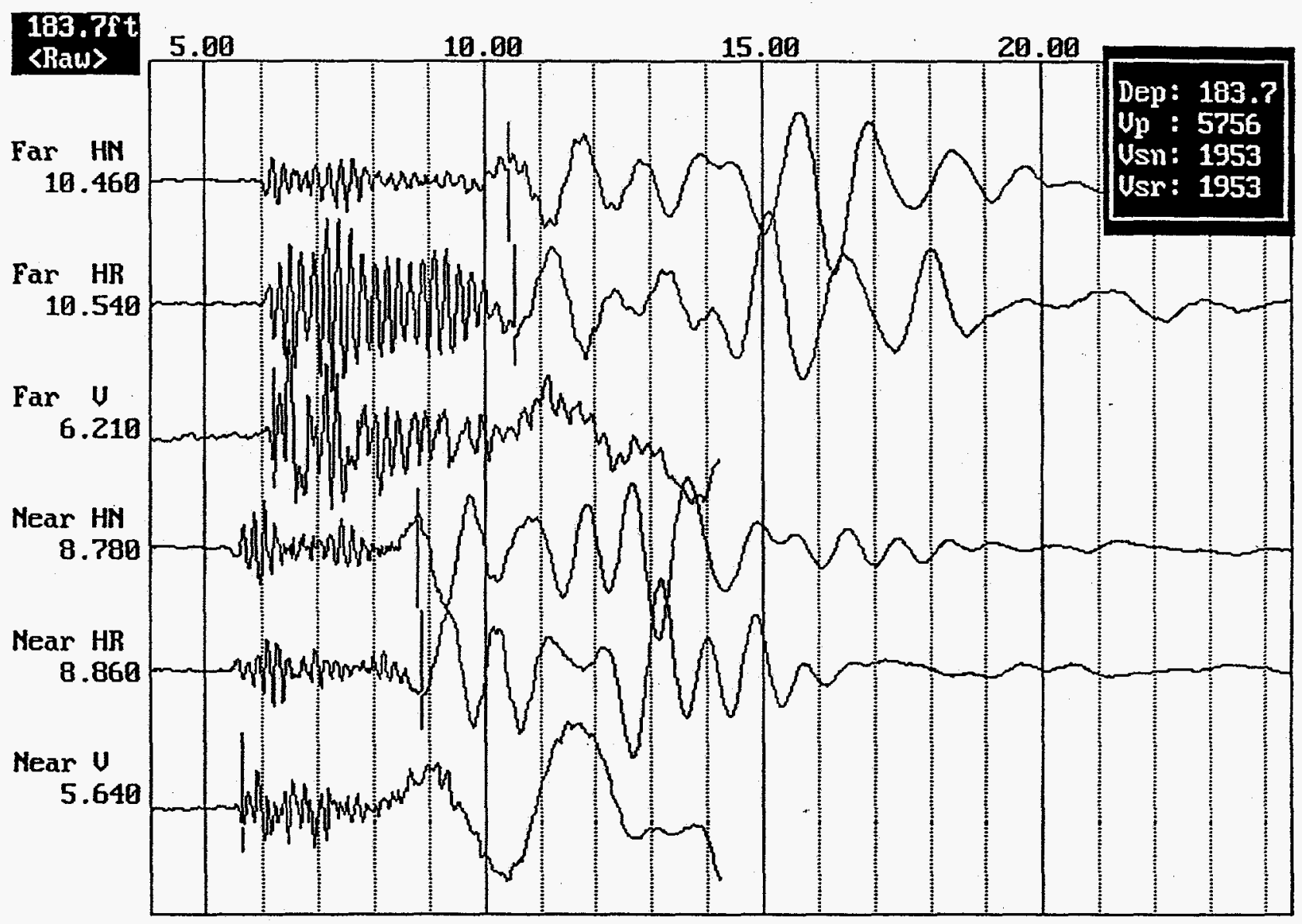

Figure 3. Unfiltered $183.7 \mathrm{ft}$ record 


\section{SRS BOREHOLE GCB-2 OYO SUSPENSION LOGGING}

P - AND S-WAVE VELOCITIES, DATA COLLECTED JUNE 30, 1996

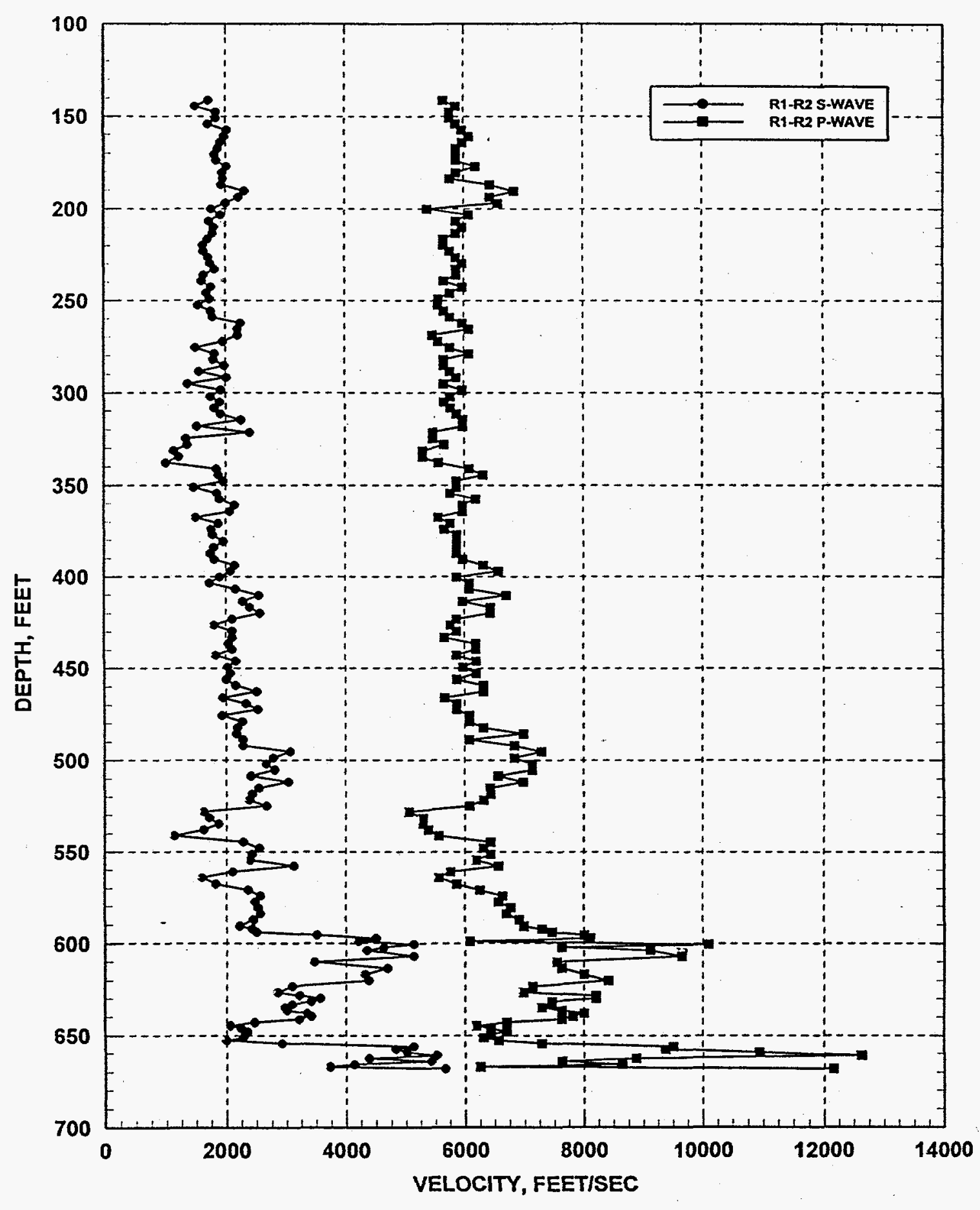

Figure 4. Suspension R1 - R2 P- and $S_{\mathrm{H}}$-wave velocities 
SRS BOREHOLE GCB-2 OYO SUSPENSION LOGGING

S-WAVE VELOCITIES, DATA COLLECTED JUNE 30, 1996

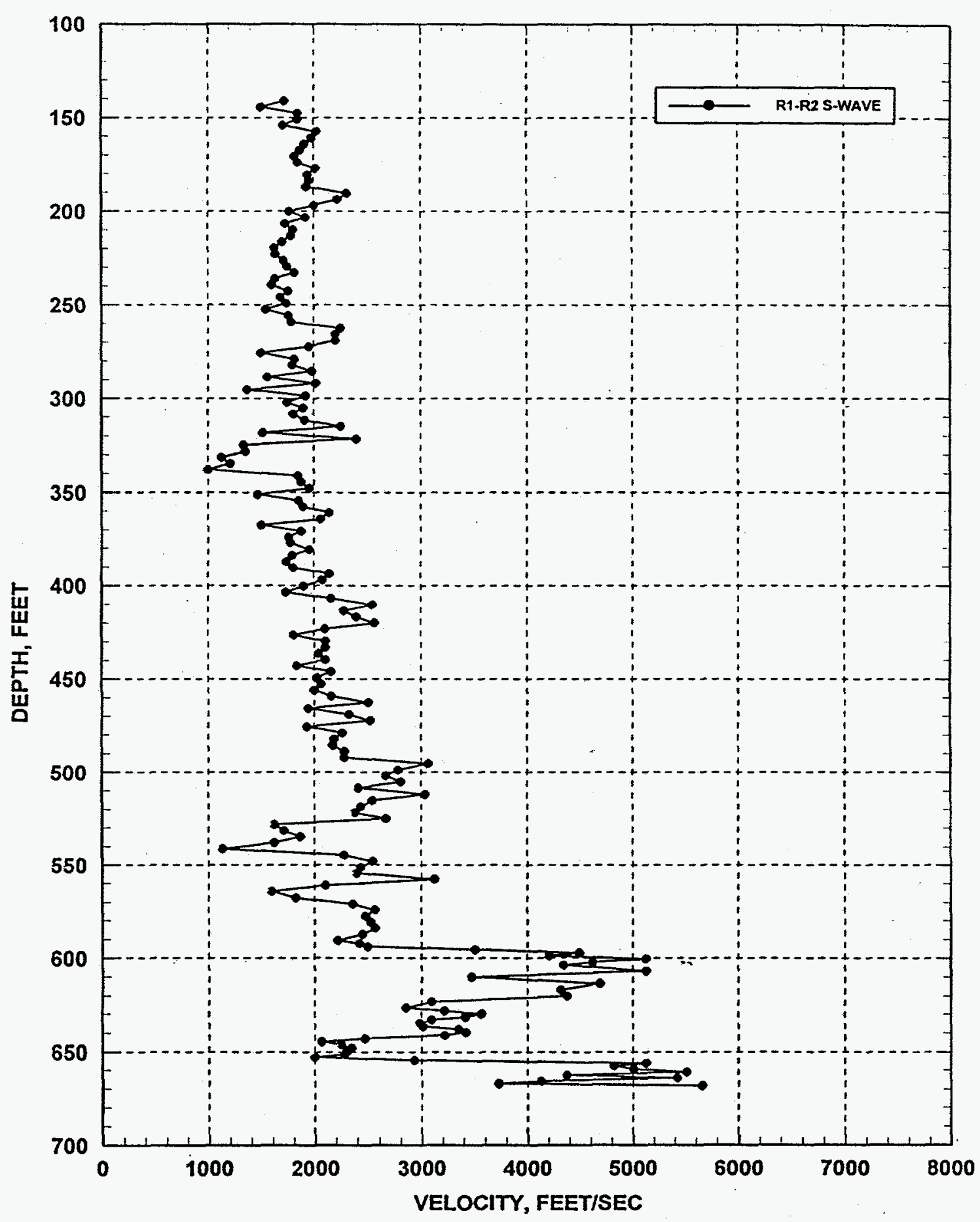

Figure 5. Suspension R1 - R2 $S_{H^{-}}$-wave velocities 


\begin{tabular}{|c|c|c|c|c|c|c|c|c|}
\hline \multirow[b]{2}{*}{$\begin{array}{l}\text { Depth } \\
\text { (feet) }\end{array}$} & \multicolumn{6}{|c|}{ Pick Times } & \multicolumn{2}{|c|}{ Velocity } \\
\hline & $\begin{array}{c}\text { Far-Hn } \\
\text { (millisec) }\end{array}$ & $\begin{array}{c}\text { Far-Hr } \\
\text { (millisec) }\end{array}$ & $\begin{array}{c}\text { Far-V } \\
\text { (millisec) }\end{array}$ & $\begin{array}{l}\text { Near-Hn } \\
\text { (millisec) }\end{array}$ & $\begin{array}{l}\text { Near-Hr } \\
\text { (millisec) }\end{array}$ & $\begin{array}{c}\text { Near-V } \\
\text { (millisec) }\end{array}$ & $\begin{array}{c}\text { Vs } \\
\text { (ft/sec) }\end{array}$ & $\begin{array}{c}V p \\
\text { (ft/sec) }\end{array}$ \\
\hline 141.1 & 11.70 & 11.78 & 6.33 & 9.80 & 9.86 & 5.75 & 1718 & 5657 \\
\hline 144.4 & 11.72 & 11.74 & 6.30 & 9.54 & 9.54 & 5.74 & 1498 & 5859 \\
\hline 147.6 & 11.24 & 11.34 & 6.27 & 9.46 & 9.56 & 5.70 & 1843 & 5756 \\
\hline 150.9 & 11.12 & 11.26 & 6.27 & 9.34 & 9.48 & 5.70 & 1843 & 5756 \\
\hline 154.2 & 11.16 & 11.26 & 6.25 & 9.24 & 9.34 & 5.69 & 1709 & 5859 \\
\hline 157.5 & 10.96 & 11.02 & 6.26 & 9.32 & 9.42 & 5.71 & 2025 & 5965 \\
\hline 160.8 & 11.06 & 11.16 & 6.25 & 9.40 & 9.50 & 5.71 & 1976 & 6076 \\
\hline 164.0 & 11.16 & 11.26 & 6.27 & 9.44 & 9.54 & 5.72 & 1907 & 5965 \\
\hline 167.3 & 11.16 & 11.24 & 6.27 & 9.40 & 9.48 & 5.71 & 1864 & 5859 \\
\hline 170.6 & 11.24 & 11.30 & 6.27 & 9.44 & 9.48 & 5.71 & 1813 & 5859 \\
\hline 173.9 & 11.06 & 11.14 & 6.24 & 9.28 & 9.36 & 5.68 & 1843 & 5859 \\
\hline 177.2 & 10.86 & 10.90 & 6.28 & 9.20 & 9.30 & 5.75 & 2013 & 6190 \\
\hline 180.5 & 10.60 & 10.68 & 6.23 & 8.90 & 9.00 & 5.67 & 1941 & 5859 \\
\hline 183.7 & 10.46 & 10.54 & 6.21 & 8.78 & 8.86 & 5.64 & 1953 & 5756 \\
\hline 187.0 & 10.36 & 10.52 & 6.16 & 8.76 & 8.72 & 5.65 & 1930 & 6433 \\
\hline 190.3 & 11.02 & 11.10 & 6.22 & 9.62 & 9.66 & 5.74 & 2310 & 6835 \\
\hline 193.6 & 11.08 & 11.16 & 6.24 & 9.60 & 9.68 & 5.73 & 2217 & 6433 \\
\hline 196.9 & 11.40 & 11.26 & 6.27 & 9.78 & 9.60 & 5.77 & 2001 & 6562 \\
\hline 200.1 & 11.54 & 11.60 & 6.30 & 9.68 & 9.74 & 5.69 & 1764 & 5378 \\
\hline 203.4 & 11.56 & 11.62 & 6.25 & 9.84 & 9.92 & 5.71 & 1919 & 6076 \\
\hline 206.7 & 11.84 & 11.90 & 6.28 & 9.94 & 10.00 & 5.72 & 1727 & 5859 \\
\hline 210.0 & 11.94 & 12.02 & 6.30 & 10.12 & 10.20 & 5.75 & 1803 & 5965 \\
\hline 213.3 & 12.06 & 12.12 & 6.33 & 10.22 & 10.28 & 5.77 & 1783 & 5859 \\
\hline 216.5 & 12.10 & 12.18 & 6.33 & 10.16 & 10.26 & 5.75 & 1700 & 5657 \\
\hline 219.8 & 12.06 & 12.14 & 6.31 & 10.04 & 10.12 & 5.73 & 1624 & 5657 \\
\hline 223.1 & 12.06 & 12.12 & 6.33 & 10.06 & 10.10 & 5.76 & 1632 & 5756 \\
\hline 226.4 & 12.04 & 12.12 & 6.36 & 10.12 & 10.20 & 5.80 & 1709 & 5859 \\
\hline 229.7 & 11.98 & 12.04 & 6.32 & 10.08 & 10.18 & 5.77 & 1745 & 5965 \\
\hline 232.9 & 12.10 & 12.18 & 6.34 & 10.28 & 10.38 & 5.78 & 1813 & 5859 \\
\hline 236.2 & 12.28 & 12.32 & 6.47 & 10.26 & 10.32 & 5.91 & 1632 & 5859 \\
\hline 239.5 & 12.48 & 12.48 & 6.37 & 10.42 & 10.44 & 5.79 & 1600 & 5657 \\
\hline 242.8 & 12.32 & 12.36 & 6.40 & 10.44 & 10.50 & 5.85 & 1754 & 5965 \\
\hline 246.1 & 12.04 & 12.08 & 6.40 & 10.08 & 10.14 & 5.83 & 1682 & 5756 \\
\hline 249.3 & 11.78 & 11.84 & 6.41 & 9.88 & 9.96 & 5.82 & 1736 & 5561 \\
\hline 252.6 & 11.38 & 11.38 & 6.32 & 9.20 & 9.30 & 5.73 & 1540 & 5561 \\
\hline 255.9 & 10.82 & 10.94 & 6.36 & 8.96 & 9.06 & 5.78 & 1754 & 5657 \\
\hline 259.2 & 11.86 & 11.96 & 6.41 & 10.02 & 10.12 & 5.84 & 1783 & 5756 \\
\hline 262.5 & 12.36 & 12.48 & 6.40 & 10.90 & 11.02 & 5.85 & 2247 & 5965 \\
\hline 265.8 & 11.78 & 11.84 & 6.41 & 10.30 & 10.34 & 5.87 & 2202 & 6076 \\
\hline 269.0 & 12.02 & 12.08 & 6.38 & 10.56 & 10.56 & 5.78 & 2202 & 5468 \\
\hline 272.3 & 12.60 & 12.68 & 6.36 & 10.94 & 10.98 & 5.77 & 1953 & 5561 \\
\hline 275.6 & 12.52 & 12.66 & 6.35 & 10.34 & 10.46 & 5.78 & 1498 & 5756 \\
\hline 278.9 & 12.16 & 12.18 & 6.35 & 10.30 & 10.42 & 5.81 & 1813 & 6076 \\
\hline 282.2 & 12.44 & 12.48 & 6.36 & 10.62 & 10.64 & 5.78 & 1793 & 5657 \\
\hline 285.4 & 12.26 & 12.32 & 6.38 & 10.58 & 10.68 & 5.80 & 1976 & 5657 \\
\hline 288.7 & 12.34 & 12.38 & 6.32 & 10.24 & 10.26 & 5.75 & 1555 & 5756 \\
\hline 292.0 & 12.00 & 12.06 & 6.31 & 10.36 & 10.44 & 5.75 & 2013 & 5859 \\
\hline 295.3 & 12.26 & 12.28 & 6.32 & 9.84 & 9.90 & 5.74 & 1367 & 5657 \\
\hline 298.6 & 11.72 & 11.80 & 6.31 & 10.00 & 10.10 & 5.76 & 1919 & 5965 \\
\hline 302.2 & 11.54 & 11.60 & 6.31 & 9.66 & 9.72 & 5.74 & 1745 & 5756 \\
\hline 305.1 & 11.32 & 11.38 & 6.30 & 9.56 & 9.68 & 5.72 & 1896 & 5657 \\
\hline 308.4 & 11.36 & 11.42 & 6.28 & 9.52 & 9.62 & 5.71 & 1803 & 5756 \\
\hline 311.7 & 11.82 & 11.92 & 6.27 & 10.12 & 10.18 & 5.71 & 1907 & 5859 \\
\hline
\end{tabular}

Table 1. Suspension R1-R2 depth, pick times, and velocities 


\begin{tabular}{|c|c|c|c|c|c|c|c|c|}
\hline \multirow[b]{2}{*}{$\begin{array}{l}\text { Depth } \\
\text { (feet) }\end{array}$} & \multicolumn{6}{|c|}{ Pick Times } & \multicolumn{2}{|c|}{ Velocity } \\
\hline & $\begin{array}{c}\text { Far-Hn } \\
\text { (millisec) }\end{array}$ & $\begin{array}{c}\mathrm{Far}-\mathrm{Hr} \\
\text { (millisec) }\end{array}$ & $\begin{array}{c}\text { Far-V } \\
\text { (millisec) }\end{array}$ & $\begin{array}{l}\text { Near-Hn } \\
\text { (millisec) }\end{array}$ & $\begin{array}{l}\text { Near-Hr } \\
\text { (millisec) }\end{array}$ & $\begin{array}{c}\text { Near-V } \\
\text { (millisec) }\end{array}$ & $\begin{array}{c}V_{s} \\
\text { (ftsec) }\end{array}$ & $\begin{array}{c}\mathrm{Vp} \\
(\mathrm{ft} / \mathrm{sec})\end{array}$ \\
\hline 315.0 & 12.92 & 12.98 & 6.32 & 11.46 & 11.52 & 5.77 & 2247 & 5965 \\
\hline 318.2 & 15.54 & 15.72 & 6.35 & 13.44 & 13.48 & 5.80 & 1512 & 5965 \\
\hline 321.5 & 15.94 & 15.98 & 6.49 & 14.60 & 14.58 & 5.89 & 2395 & 5468 \\
\hline 324.8 & 16.64 & 16.72 & 6.48 & 14.18 & 14.26 & 5.88 & 1334 & 5468 \\
\hline 328.1 & 16.02 & 16.10 & 6.46 & 13.62 & 13.64 & 5.88 & 1350 & 5657 \\
\hline 331.4 & 15.28 & 15.44 & 6.47 & 12.44 & 12.46 & 5.85 & 1127 & 5292 \\
\hline 334.7 & 14.28 & 14.32 & 6.37 & 11.52 & 11.66 & 5.75 & 1211 & 5292 \\
\hline 337.9 & 13.66 & 13.72 & 6.32 & 10.42 & 10.40 & 5.73 & 1000 & 5561 \\
\hline 341.2 & 11.96 & 12.00 & 6.28 & 10.18 & 10.22 & 5.74 & 1843 & 6076 \\
\hline 344.5 & 11.94 & 11.98 & 6.29 & 10.18 & 10.24 & 5.77 & 1875 & 6309 \\
\hline 347.8 & 11.70 & 11.76 & 6.29 & 9.98 & 10.12 & 5.73 & 1953 & 5859 \\
\hline 351.1 & 11.86 & 11.98 & 6.28 & 9.60 & 9.76 & 5.72 & 1465 & 5859 \\
\hline 354.3 & 11.66 & 11.72 & 6.29 & 9.88 & 9.96 & 5.72 & 1854 & 5756 \\
\hline 357.6 & 11.44 & 11.50 & 6.28 & 9.68 & 9.80 & 5.75 & 1896 & 6190 \\
\hline 360.9 & 11.42 & 11.50 & 6.30 & 9.90 & 9.96 & 5.75 & 2144 & 5965 \\
\hline 364.2 & 11.86 & 11.92 & 6.31 & 10.26 & 10.34 & 5.76 & 2063 & 5965 \\
\hline 367.5 & 11.94 & 12.02 & 6.32 & 9.74 & 9.84 & 5.73 & 1498 & 5561 \\
\hline 370.7 & 11.58 & 11.66 & 6.29 & 9.82 & 9.92 & 5.72 & 1875 & 5756 \\
\hline 374.0 & 11.72 & 11.80 & 6.44 & 9.86 & 9.92 & 5.86 & 1754 & 5657 \\
\hline 377.0 & 11.68 & 11.74 & 6.37 & 9.82 & 9.90 & 5.81 & 1773 & 5859 \\
\hline 380.6 & 11.40 & 11.48 & 6.36 & 9.72 & $9: 80$ & 5.80 & 1953 & 5859 \\
\hline 383.9 & 11.34 & 11.42 & 6.29 & 9.52 & 9.58 & 5.73 & 1793 & 5859 \\
\hline 387.1 & 11.26 & 11.30 & 6.27 & 9.36 & 9.42 & 5.71 & 1736 & 5859 \\
\hline 390.4 & 11.18 & 11.20 & 6.31 & 9.34 & 9.40 & 5.76 & 1803 & 5965 \\
\hline 393.7 & 10.86 & 10.88 & 6.29 & 9.32 & 9.36 & 5.77 & 2144 & 6309 \\
\hline 397.0 & 10.70 & 10.78 & 6.24 & 9.12 & 9.20 & 5.74 & 2076 & 6562 \\
\hline 400.3 & 10.46 & 10.54 & 6.22 & 8.74 & 8.80 & 5.66 & 1896 & 5859 \\
\hline 403.5 & 10.20 & 10.24 & 6.17 & 8.28 & 8.36 & 5.63 & 1727 & 6076 \\
\hline 406.8 & 9.68 & 9.76 & 6.15 & 8.16 & 8.24 & 5.61 & 2158 & 6076 \\
\hline 410.1 & 9.66 & 9.76 & 6.10 & 8.38 & 8.46 & 5.61 & 2543 & 6696 \\
\hline 413.4 & 9.98 & 10.08 & 6.17 & 8.54 & 8.64 & 5.62 & 2278 & 5965 \\
\hline 416.7 & 10.06 & 10.18 & 6.19 & 8.70 & $8.80^{\circ}$ & 5.68 & 2395 & 6433 \\
\hline 420.0 & 10.32 & 10.40 & 6.22 & 9.04 & 9.12 & 5.71 & 2563 & 6433 \\
\hline 423.2 & 10.58 & 10.68 & 6.27 & 9.02 & 9.12 & 5.71 & 2103 & 5859 \\
\hline 426.5 & 10.68 & 10.76 & 6.27 & 8.86 & 8.94 & 5.70 & 1803 & 5756 \\
\hline 429.8 & 10.64 & 10.72 & 6.26 & 9.08 & 9.16 & 5.70 & 2103 & 5859 \\
\hline 433.1 & 10.72 & 10.82 & 6.26 & 9.16 & 9.26 & 5.68 & 2103 & 5657 \\
\hline 436.4 & 10.72 & 10.82 & 6.21 & 9.10 & 9.22 & 5.68 & 2038 & 6190 \\
\hline 439.6 & 10.80 & 10.88 & 6.22 & 9.22 & 9.34 & $5: 69$ & 2103 & 6190 \\
\hline 442.9 & 10.76 & 10.88 & 6.23 & 8.96 & 9.10 & 5.67 & 1833 & 5859 \\
\hline 446.2 & 10.64 & 10.72 & 6.23 & 9.12 & 9.20 & 5.70 & 2158 & 6190 \\
\hline 449.5 & 10.48 & 10.56 & 6.21 & 8.86 & 8.94 & 5.66 & 2025 & 5965 \\
\hline 452.8 & 10.48 & 10.54 & 6.18 & 8.90 & 8.94 & 5.65 & 2063 & 6190 \\
\hline 456.0 & 10.60 & 10.64 & 6.22 & 8.96 & 9.00 & 5.66 & 2001 & 5859 \\
\hline 459.3 & 10.48 & 10.54 & 6.18 & 8.96 & 9.02 & 5.66 & 2158 & 6309 \\
\hline 462.6 & 10.64 & 10.72 & 6.36 & 9.34 & 9.40 & 5.84 & 2504 & 6309 \\
\hline 465.9 & 10.72 & 10.76 & 6.37 & 9.02 & 9.08 & 5.79 & 1941 & 5657 \\
\hline 469.2 & 10.44 & 10.48 & 6.31 & 9.00 & 9.10 & 5.75 & 2327 & 5859 \\
\hline 472.4 & 10.28 & 10.34 & 6.29 & 8.98 & 9.04 & 5.73 & 2524 & 5859 \\
\hline 475.7 & 10.24 & 10.34 & 6.29 & 8.54 & 8.64 & 5.75 & 1930 & 6076 \\
\hline 479.0 & 10.16 & 10.22 & 6.28 & 8.70 & 8.78 & 5.74 & 2263 & 6076 \\
\hline 482.3 & 9.82 & 9.88 & 6.10 & 8.32 & 8.38 & 5.58 & 2187 & 6309 \\
\hline 485.6 & 9.52 & 9.60 & 6.16 & 8.00 & 8.10 & 5.69 & 2173 & 6981 \\
\hline
\end{tabular}

Table 1 continued. Suspension R1-R2 depth, pick times, and velocities 


\begin{tabular}{|c|c|c|c|c|c|c|c|c|}
\hline \multirow[b]{2}{*}{$\begin{array}{l}\text { Depth } \\
\text { (feet) }\end{array}$} & \multicolumn{6}{|c|}{ Pick Times } & \multicolumn{2}{|c|}{ Velocity } \\
\hline & $\begin{array}{c}\text { Far-Hn } \\
\text { (millisec) }\end{array}$ & $\begin{array}{c}\text { Far-Hr } \\
\text { (millisec) }\end{array}$ & $\begin{array}{c}\text { Far-V } \\
\text { (millisec) }\end{array}$ & $\begin{array}{l}\text { Near-Hn } \\
\text { (millisec) }\end{array}$ & $\begin{array}{l}\text { Near-Hr } \\
\text { (millisec) }\end{array}$ & $\begin{array}{c}\text { Near-V } \\
\text { (millisec) }\end{array}$ & $\begin{array}{c}\text { Vs } \\
\text { (ft/sec) }\end{array}$ & $\begin{array}{c}V p \\
(\mathrm{ft} / \mathrm{sec})\end{array}$ \\
\hline 488.9 & 9.16 & 9.42 & 6.07 & 7.72 & 7.98 & 5.53 & 2278 & 6076 \\
\hline 492.1 & 8.94 & 9.02 & 5.98 & 7.50 & 7.58 & 5.50 & 2278 & 6835 \\
\hline 495.4 & 8.88 & 8.96 & 6.01 & 7.82 & 7.88 & 5.56 & 3066 & 7291 \\
\hline 498.7 & 8.94 & 9.04 & 5.95 & 7.76 & 7.86 & 5.47 & 2780 & 6835 \\
\hline $502.0^{\circ}$ & 8.92 & 9.00 & 5.99 & 7.68 & 7.78 & 5.53 & 2667 & 7132 \\
\hline 505.3 & 9.06 & 9.18 & 5.95 & 7.90 & 8.00 & 5.49 & 2804 & 7132 \\
\hline 508.5 & 9.26 & 9.34 & 6.05 & 7.90 & 7.98 & 5.55 & 2412 & 6562 \\
\hline 511.8 & 9.46 & 9.54 & 6.07 & 8.40 & 8.44 & 5.60 & 3038 & 6981 \\
\hline 515.1 & 10.00 & 10.04 & 6.13 & 8.70 & 8.76 & 5.62 & 2543 & 6433 \\
\hline 518.4 & 10.98 & 11.06 & 6.21 & 9.64 & 9.70 & 5.70 & 2430 & 6433 \\
\hline 521.7 & 11.12 & 11.22 & 6.26 & 9.74 & 9.84 & 5.74 & 2377 & 6309 \\
\hline 524.9 & 12.52 & 12.40 & 6.34 & 11.30 & 11.16 & 5.80 & 2667 & 6076 \\
\hline 528.2 & 12.76 & 12.90 & 6.51 & 10.72 & 10.88 & 5.86 & 1616 & 5047 \\
\hline 531.5 & 12.06 & 12.10 & 6.49 & 10.14 & 10.18 & 5.87 & 1709 & 5292 \\
\hline 534.8 & 12.28 & 12.24 & 6.38 & 10.46 & 10.54 & 5.76 & 1864 & 5292 \\
\hline 538.1 & 11.76 & 11.82 & 6.26 & 9.72 & 9.80 & 5.65 & 1616 & 5378 \\
\hline 541.3 & 11.16 & 11.20 & 6.17 & 8.26 & 8.30 & 5.58 & 1131 & 5561 \\
\hline 544.6 & 9.76 & 9.86 & 6.32 & 8.36 & 8.38 & 5.81 & 2278 & 6433 \\
\hline 547.9 & 10.02 & 10.10 & 6.21 & 8.76 & 8.78 & 5.69 & 2543 & 6309 \\
\hline 551.2 & 11.54 & 11.62 & 6.27 & 10.20 & 10.26 & 5.76 & 2430 & 6433 \\
\hline 554.5 & 12.68 & 12.76 & 6.27 & 11.30 & 11.40 & 5.74 & 2395 & 6190 \\
\hline 557.7 & 11.10 & 11.12 & 6.25 & 10.04 & 10.08 & 5.75 & 3125 & 6562 \\
\hline 561.0 & 11.12 & 11.12 & 6.27 & 9.56 & 9.56 & 5.70 & 2103 & 5756 \\
\hline 564.3 & 10.62 & 12.54 & 6.29 & 9.48 & 9.56 & 5.70 & 1593 & 5561 \\
\hline 567.6 & 10.06 & 10.12 & 6.22 & 8.26 & 8.32 & 5.66 & 1823 & 5859 \\
\hline 570.9 & 9.44 & 9.56 & 6.29 & 8.06 & 8.16 & 5.77 & 2360 & 6249 \\
\hline 574.2 & 9.27 & 9.38 & 6.25 & 8.00 & 8.09 & 5.75 & 2563 & 6628 \\
\hline 577.4 & 9.39 & 9.50 & 6.18 & 8.08 & 8.16 & 5.68 & 2476 & 6562 \\
\hline 580.7 & 9.28 & 9.35 & 6.14 & 7.98 & 8.05 & 5.65 & 2524 & 6765 \\
\hline 584.0 & 9.07 & 9.15 & 6.14 & 7.79 & 7.87 & 5.65 & 2563 & 6696 \\
\hline 587.3 & 8.46 & 8.54 & 6.01 & 7.12 & 7.20 & 5.54 & 2448 & 6907 \\
\hline 590.6 & 8.69 & 8.82 & 5.71 & 7.22 & 7.33 & 5.24 & 2217 & 6981 \\
\hline 592.2 & 8.26 & 8.50 & 5.63 & 6.93 & 7.12 & 5.18 & 2421 & 7291 \\
\hline 593.8 & 8.06 & 8.24 & 5.58 & 6.75 & 6.92 & 5.14 & 2495 & 7456 \\
\hline 595.5 & 7.01 & 7.12 & 5.54 & 6.10 & 6.16 & 5.13 & 3509 & 8002 \\
\hline 597.1 & 6.88 & 6.92 & 5.64 & 6.15 & 6.19 & 5.24 & 4494 & 8101 \\
\hline 598.8 & 6.89 & 8.17 & 5.60 & 6.20 & 7.30 & 5.06 & 4206 & 6076 \\
\hline 600.4 & 6.93 & 7.01 & 5.53 & 6.29 & 6.37 & 5.20 & 5126 & 10095 \\
\hline 602.0 & 6.91 & 6.99 & 5.44 & 6.20 & 6.28 & 5.01 & 4621 & 7630 \\
\hline 603.7 & 6.93 & 6.99 & 5.45 & 6.17 & 6.24 & 5.09 & 4345 & 9113 \\
\hline 607.0 & 7.03 & 7.03 & 5.56 & 6.38 & 6.40 & 5.22 & 5126 & 9650 \\
\hline 610.2 & 7.16 & 7.23 & 5.63 & 6.21 & 6.29 & 5.19 & 3472 & 7542 \\
\hline 613.5 & 7.44 & 7.46 & 5.69 & 6.70 & 6.80 & 5.26 & 4687 & 7630 \\
\hline 616.8 & 7.80 & 7.90 & 5.74 & 7.04 & 7.14 & 5.33 & 4317 & 8002 \\
\hline 620.1 & 8.00 & 8.10 & 5.94 & 7.24 & 7.36 & 5.55 & 4374 & 8412 \\
\hline 623.4 & 8.40 & 8.46 & 5.87 & 7.34 & 7.40 & 5.41 & 3095 & 7132 \\
\hline 626.6 & 8.36 & 8.44 & 5.88 & 7.22 & 7.28 & 5.41 & 2853 & 6981 \\
\hline 628.3 & 8.26 & 8.34 & 5.84 & 7.24 & 7.32 & 5.44 & 3217 & 8202 \\
\hline 629.9 & 8.34 & 8.42 & 5.84 & 7.42 & 7.50 & 5.44 & 3566 & 8202 \\
\hline 631.6 & 8.46 & 8.54 & 5.88 & 7.50 & 7.58 & 5.44 & 3418 & 7456 \\
\hline 633.2 & 8.80 & 8.84 & 5.91 & 7.72 & 7.80 & 5.47 & 3095 & 7456 \\
\hline 634.8 & 8.86 & 8.94 & 5.94 & 7.76 & 7.84 & 5.49 & 2983 & 7291 \\
\hline 636.5 & 8.98 & 9.06 & 5.97 & 7.90 & 7.96 & 5.54 & 3010 & 7630 \\
\hline
\end{tabular}

Table 1 continued. Suspension R1-R2 depth, pick times, and velocities 


\begin{tabular}{|c|c|c|c|c|c|c|c|c|}
\hline \multirow{2}{*}{$\begin{array}{c}\text { Depth } \\
\text { (feet) }\end{array}$} & \multicolumn{9}{|c|}{$\begin{array}{c}\text { Far-Hn } \\
\text { (millisec) }\end{array}$} & $\begin{array}{c}\text { Far-Hr } \\
\text { (millisec) }\end{array}$ & $\begin{array}{c}\text { Far-V } \\
\text { (millisec) }\end{array}$ & $\begin{array}{c}\text { Near-Hn } \\
\text { (millisec) }\end{array}$ & $\begin{array}{c}\text { Near-Hr } \\
\text { (millisec) }\end{array}$ & $\begin{array}{c}\text { Near-V } \\
\text { (millisec) }\end{array}$ & $\begin{array}{c}\text { Vs } \\
\text { (ftsec) }\end{array}$ & $\begin{array}{c}\text { Vp } \\
\text { (ft/sec) }\end{array}$ \\
\hline 638.1 & 9.16 & 9.22 & 5.99 & 8.16 & 8.26 & 5.58 & 3348 & 8002 \\
639.8 & 9.42 & 9.44 & 6.06 & 8.44 & 8.50 & 5.64 & 3418 & 7812 \\
641.4 & 9.78 & 9.80 & 6.13 & 8.74 & 8.80 & 5.70 & 3217 & 7630 \\
643.0 & 9.94 & 10.02 & 6.17 & 8.64 & 8.66 & 5.68 & 2467 & 6696 \\
644.7 & 9.72 & 9.82 & 6.16 & 8.16 & 8.20 & 5.63 & 2063 & 6190 \\
646.3 & 9.22 & 9.34 & 5.98 & 7.80 & 7.84 & 5.47 & 2247 & 6433 \\
648.0 & 8.78 & 8.84 & 5.85 & 7.42 & 7.40 & 5.36 & 2343 & 6696 \\
649.6 & 8.48 & 8.52 & 5.78 & 7.06 & 7.10 & 5.27 & 2310 & 6433 \\
651.3 & 7.98 & 8.10 & 5.80 & 6.56 & 6.64 & 5.28 & 2278 & 6309 \\
652.9 & 7.65 & 7.67 & 5.71 & 6.00 & 6.04 & 5.21 & 2001 & 6562 \\
654.5 & 7.13 & 7.10 & 5.51 & 5.98 & 6.01 & 5.06 & 2929 & 7291 \\
656.2 & 6.49 & 6.60 & 5.57 & 5.87 & 5.94 & 5.22 & 5126 & 9510 \\
657.8 & 6.45 & 6.48 & 5.28 & 5.76 & 5.81 & 4.93 & 4825 & 9374 \\
659.5 & 6.44 & 6.47 & 5.27 & 5.77 & 5.83 & 4.97 & 5009 & 10936 \\
661.1 & 6.35 & 6.42 & 5.27 & 5.76 & 5.82 & 5.01 & 5514 & 12619 \\
662.7 & 6.41 & 6.40 & 5.18 & 5.62 & 5.69 & 4.81 & 4374 & 8867 \\
664.4 & 6.36 & 6.41 & 5.43 & 5.72 & 5.84 & 5.00 & 5423 & 7630 \\
666.0 & 6.57 & 6.64 & 5.33 & 5.77 & 5.85 & 4.95 & 4127 & 8634 \\
667.3 & 6.49 & 6.32 & 5.42 & 5.51 & 5.54 & 4.90 & 3728 & 6249 \\
668.3 & 6.05 & 6.10 & 5.24 & 5.47 & 5.52 & 4.97 & 5657 & 12151 \\
\hline
\end{tabular}

Table 1 continued. Suspension R1-R2 depth, pick times, and velocities 


\section{APPENDIX A}

\section{SUSPENSION VELOCITY MEASUREMENT \\ QUALITY ASSURANCE SUSPENSION SOURCE \\ TO RECEIVER ANALYSIS RESULTS}




\section{SRS BOREHOLE GCB-2 OYO SUSPENSION LOGGING}

$P$ - AND S-WAVE VELOCITIES, DATA COLLECTED JUNE 30, 1996

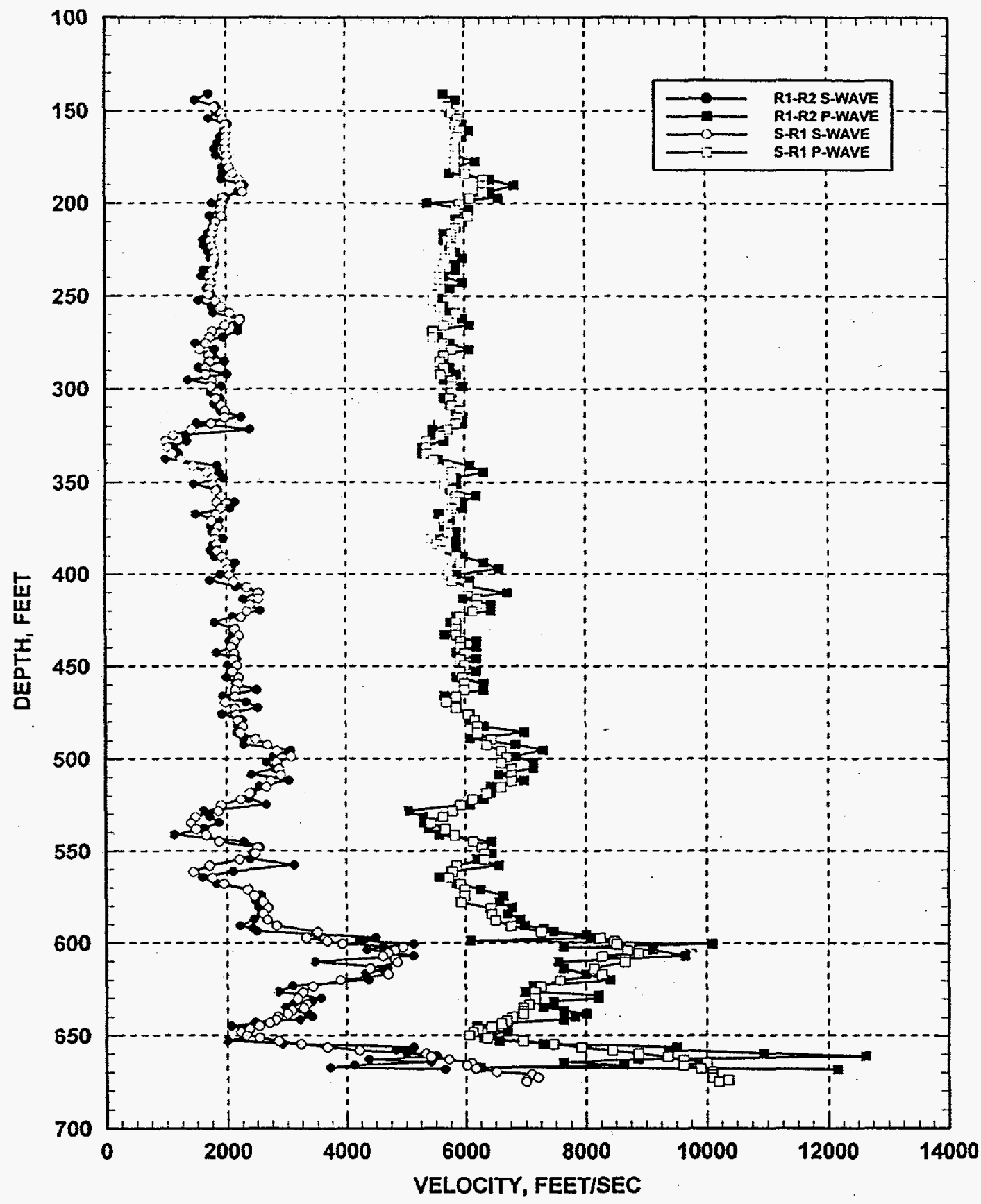

Figure A-1. R1 - R2 high resolution analysis and S-R1 quality assurance analysis $\mathrm{P}$-.and $\mathrm{S}_{\mathrm{H}}$-wave data 


\section{SRS BOREHOLE GCB-2 OYO SUSPENSION LOGGING}

S-WAVE VELOCITIES, DATA COLLECTED JUNE 30, 1996

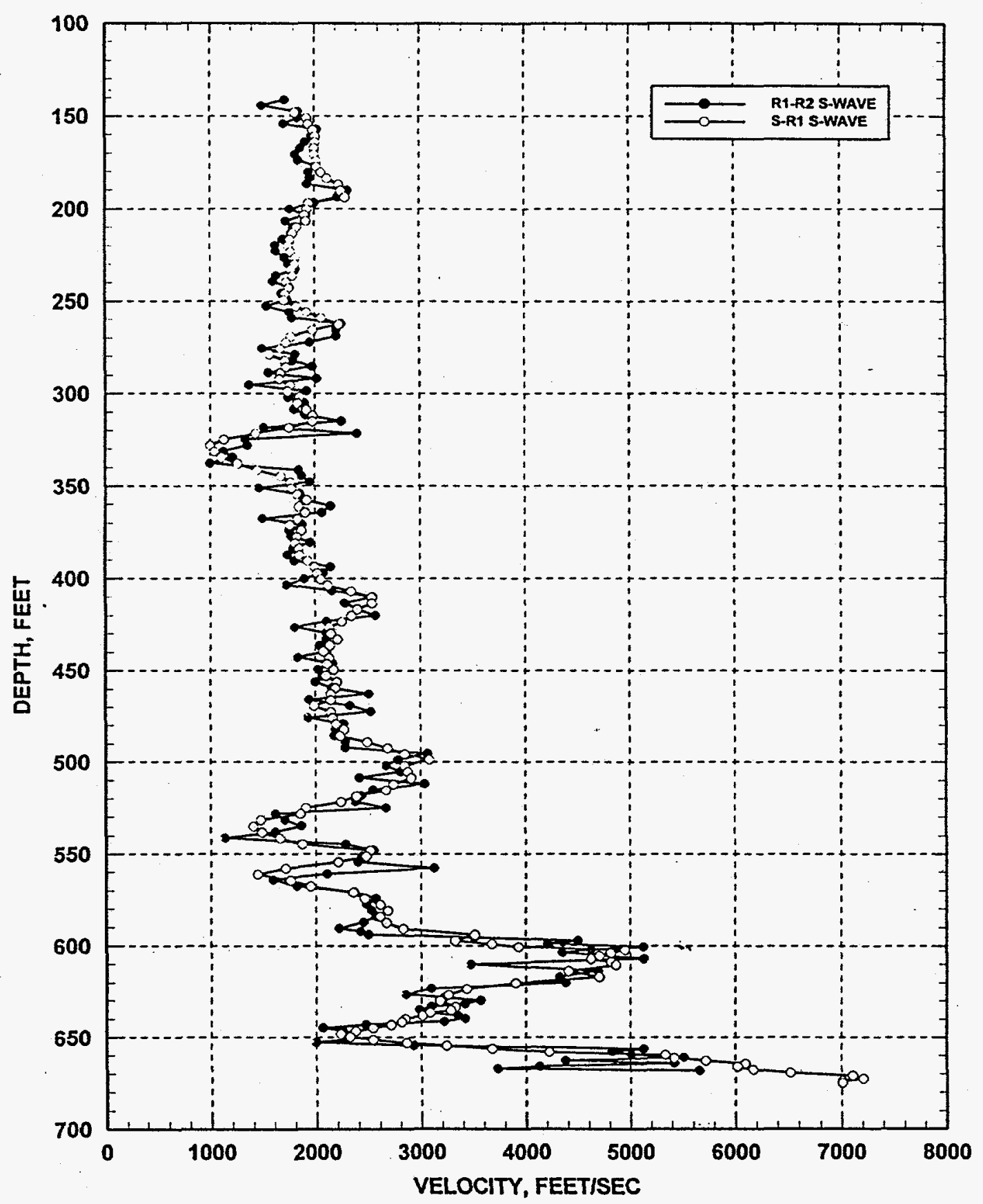

Figure A-2. R1 - R2 high resolution analysis and S-R1 quality assurance $S_{\mathrm{H}}$-wave data 


\begin{tabular}{|c|c|c|}
\hline DeptI & J-R & S-RT \\
(feet) & vs (tt/sec) & V (ftsec) \\
\hline 147.07 & 1813 & 5722 \\
151.15 & 1929 & 5787 \\
154.43 & 1943 & 5886 \\
157.71 & 1988 & 5852 \\
160.99 & 2012 & 5920 \\
164.27 & 2004 & 5886 \\
167.55 & 1996 & 5852 \\
170.83 & 1996 & 5852 \\
174.11 & 2020 & 5852 \\
177.39 & 2020 & 5852 \\
180.67 & 2060 & 5852 \\
183.96 & 2119 & 6023 \\
187.24 & 2229 & 6319 \\
190.52 & 2249 & 6319 \\
193.80 & 2289 & 6319 \\
197.08 & 1943 & 6095 \\
200.36 & 1929 & 5920 \\
203.64 & 1907 & 5852 \\
206.92 & 1922 & 6059 \\
210.20 & 1833 & 5920 \\
213.48 & 1794 & 5852 \\
216.76 & 1770 & 5787 \\
220.04 & 1752 & 5722 \\
223.33 & 1776 & 5787 \\
226.61 & 1813 & 5787 \\
229.89 & 1813 & 5691 \\
233.17 & 1776 & 5659 \\
236.45 & 1788 & 5598 \\
239.73 & 1728 & 5598 \\
243.01 & 1752 & 5598 \\
246.29 & 1717 & 5598 \\
249.57 & 1705 & 5479 \\
252.85 & 1826 & 5479 \\
256.13 & 1922 & 5598 \\
259.41 & 2060 & 5852 \\
262.70 & 2229 & 5722 \\
265.98 & 1981 & 5659 \\
269.26 & 1776 & 5479 \\
272.54 & 1728 & 5479 \\
275.82 & 1672 & 5659 \\
279.10 & 1570 & 5787 \\
282.38 & 1717 & 5659 \\
285.66 & 1728 & 5598 \\
288.94 & 1672 & 5659 \\
292.22 & 1661 & 5598 \\
295.50 & 1764 & 5787 \\
298.78 & 1740 & 5787 \\
302.07 & 1853 & 5787 \\
305.35 & 1833 & 5754 \\
308.96 & 1922 & 5787 \\
311.91 & 1981 & 5920 \\
315.19 & 1977 & 5886 \\
318.47 & 1752 & 5852 \\
321.75 & 1431 & 5722 \\
325.03 & 1132 & 5598 \\
328.31 & 1002 & 5365 \\
331.59 & 1040 & 5365 \\
334.87 & 1108 & 5365 \\
338.15 & 1262 & 5479 \\
341.44 & 1451 & 5722 \\
\hline
\end{tabular}

\begin{tabular}{|c|c|c|}
\hline $\begin{array}{l}\text { DeptI } \\
\text { (feet) }\end{array}$ & $\begin{array}{l}\text { S-FT (ft/sec) } \\
\text { Vs }\end{array}$ & $\begin{array}{c}\text { S-RT } \\
V_{p}(f / s e c)\end{array}$ \\
\hline 344.72 & 7018 & गम्ठा \\
\hline 348.00 & 1770 & 5787 \\
\hline 351.28 & 1776 & 5659 \\
\hline 354.56 & 1826 & 5787 \\
\hline 357.84 & 1922 & 5852 \\
\hline 361.12 & 1846 & 5852 \\
\hline 364.40 & 1907 & 5787 \\
\hline 367.68 & 1833 & 5722 \\
\hline 370.96 & 1758 & 5659 \\
\hline 374.24 & 1873 & 5722 \\
\hline 377.52 & 1826 & 5722 \\
\hline 380.81 & 1810 & 5450 \\
\hline 383.76 & 1846 & 5538 \\
\hline 387.37 & 1853 & 5598 \\
\hline 390.65 & 1929 & 5852 \\
\hline 393.93 & 1996 & 5920 \\
\hline 397.21 & 2020 & 5722 \\
\hline 400.49 & 2052 & 5722 \\
\hline 403.77 & 2119 & 5787 \\
\hline 407.05 & 2341 & 6059 \\
\hline 410.33 & 2537 & 6205 \\
\hline 413.61 & 2537 & 6205 \\
\hline 416.89 & 2395 & 6205 \\
\hline 420.18 & 2341 & 6131 \\
\hline 423.46 & 2249 & 5920 \\
\hline 426.74 & 2128 & 5852 \\
\hline 430.02 & 2146 & 5852 \\
\hline 433.30 & 2210 & 5852 \\
\hline 436.58 & 2137 & 5920 \\
\hline 439.86 & 2077 & 5920 \\
\hline 443.14 & 2128 & 5988 \\
\hline 446.42 & 2111 & 5920 \\
\hline 449.70 & 2173 & 5988 \\
\hline 452.98 & 2102 & 5920 \\
\hline 456.27 & 2206 & 5954 . \\
\hline 459.55 & 2191 & 5988 \\
\hline 462.83 & 2146 & 5988 \\
\hline 466.11 & 2146 & 5852 \\
\hline 469.39 & 1988 & 5691 \\
\hline 472.67 & 2146 & 5852 \\
\hline 475.95 & 2164 & 6059 \\
\hline 479.23 & 2191 & 6168 \\
\hline 482.51 & 2269 & 6205 \\
\hline 485.79 & 2229 & 6205 \\
\hline 489.07 & 2488 & 6438 \\
\hline 492.35 & 2682 & 6358 \\
\hline 495.64 & 2845 & 6603 \\
\hline 498.92 & 3084 & 6688 \\
\hline 502.20 & 2845 & 6603 \\
\hline 505.48 & 2877 & 6776 \\
\hline 508.76 & 2910 & 6776 \\
\hline 512.04 & 2739 & 6776 \\
\hline 515.32 & 2668 & 6603 \\
\hline 518.60 & 2384 & 6358 \\
\hline 521.88 & 2239 & 6131 \\
\hline 525.16 & 1907 & 5920 \\
\hline 528.44 & 1859 & 5787 \\
\hline 531.72 & 1478 & 5628 \\
\hline 535.01 & 1407 & 5421 \\
\hline 538.29 & 1488 & 5659 \\
\hline
\end{tabular}

\begin{tabular}{|c|c|c|}
\hline $\begin{array}{l}\text { Dephit } \\
\text { (feet) }\end{array}$ & $\begin{array}{c}s-k 1 \\
V s \text { (ft/sec) }\end{array}$ & $\begin{array}{c}S-k T \\
V p(f t / s e c)\end{array}$ \\
\hline 341.51 & T1009 & 3815 \\
\hline 544.85 & 1873 & 6131 \\
\hline 548.13 & 2512 & 6280 \\
\hline 551.41 & 2476 & 6319 \\
\hline 554.69 & 2210 & 6319 \\
\hline 557.97 & 1711 & 5852 \\
\hline 561.25 & 1443 & 5787 \\
\hline 564.53 & 1758 & 5722 \\
\hline 567.81 & 1951 & 5920 \\
\hline 571.09 & 2352 & 5988 \\
\hline 574.38 & 2458 & 5988 \\
\hline 577.66 & 2608 & 5920 \\
\hline 580.94 & 2682 & 6417 \\
\hline 584.22 & 2608 & 6438 \\
\hline 587.50 & 2668 & 6498 \\
\hline 590.78 & 2830 & 6754 \\
\hline 594.06 & 3515 & 7254 \\
\hline 597.34 & 3323 & 8240 \\
\hline 598.98 & 3679 & 8477 \\
\hline 600.62 & 3931 & 8512 \\
\hline 602.26 & 4952 & 8729 \\
\hline 603.90 & 4813 & 8692 \\
\hline 605.54 & 4703 & 8879 \\
\hline 607.18 & 4619 & 8273 \\
\hline 608.82 & 4813 & 8655 \\
\hline 610.46 & 4858 & 8655 \\
\hline 613.75 & 4402 & 8142 \\
\hline 617.03 & 4703 & 8273 \\
\hline 620.31 & 3902 & 7574 \\
\hline 623.59 & 3433 & 7254 \\
\hline 626.87 & 3259 & 7153 \\
\hline 630.15 & 3179 & 7153 \\
\hline 633.43 & 3323 & 7055 \\
\hline 635.07 & 3280 & 6959 \\
\hline 636.71 & 3084 & 6959 \\
\hline 638.35 & 3012 & 6959 \\
\hline 639.99 & 2845 & 6776 \\
\hline 641.63 & 2814 & 6688 \\
\hline 643.27 & 2711 & 6603 \\
\hline 644.91 & 2537 & 6438 \\
\hline 646.55 & 2373 & 6205 \\
\hline 648.19 & 2229 & 6131 \\
\hline 649.83 & 2320 & 6059 \\
\hline 651.48 & 2537 & 6358 \\
\hline 653.12 & 2861 & 6959 \\
\hline 654.76 & 3239 & 7464 \\
\hline 656.40 & 3679 & 7923 \\
\hline 658.04 & 4221 & 8443 \\
\hline 659.68 & 5337 & 8879 \\
\hline 661.32 & 5421 & 9364 \\
\hline 662.96 & 5722 & 9626 \\
\hline 664.60 & 6095 & 10000 \\
\hline 666.24 & 6023 & 9626 \\
\hline 667.88 & 6168 & 9904 \\
\hline 669.52 & 6519 & 10098 \\
\hline 671.16 & 7103 & 10098 \\
\hline 672.80 & 7203 & 10098 \\
\hline 674.11 & 7007 & 10352 \\
\hline 675.10 & 7007 & 10198 \\
\hline
\end{tabular}

Table A-1. S - R1 quality assurance analysis $\mathrm{P}$ - and $\mathrm{S}_{\mathrm{H}}$-wave data 


\section{APPENDIX B}

\section{SUSPENSION $\mathbf{S}_{\mathrm{H}}$-WAVE R1 WAVEFORM RECORDS WITH S - R1 FIRST ARRIVAL PICKS}




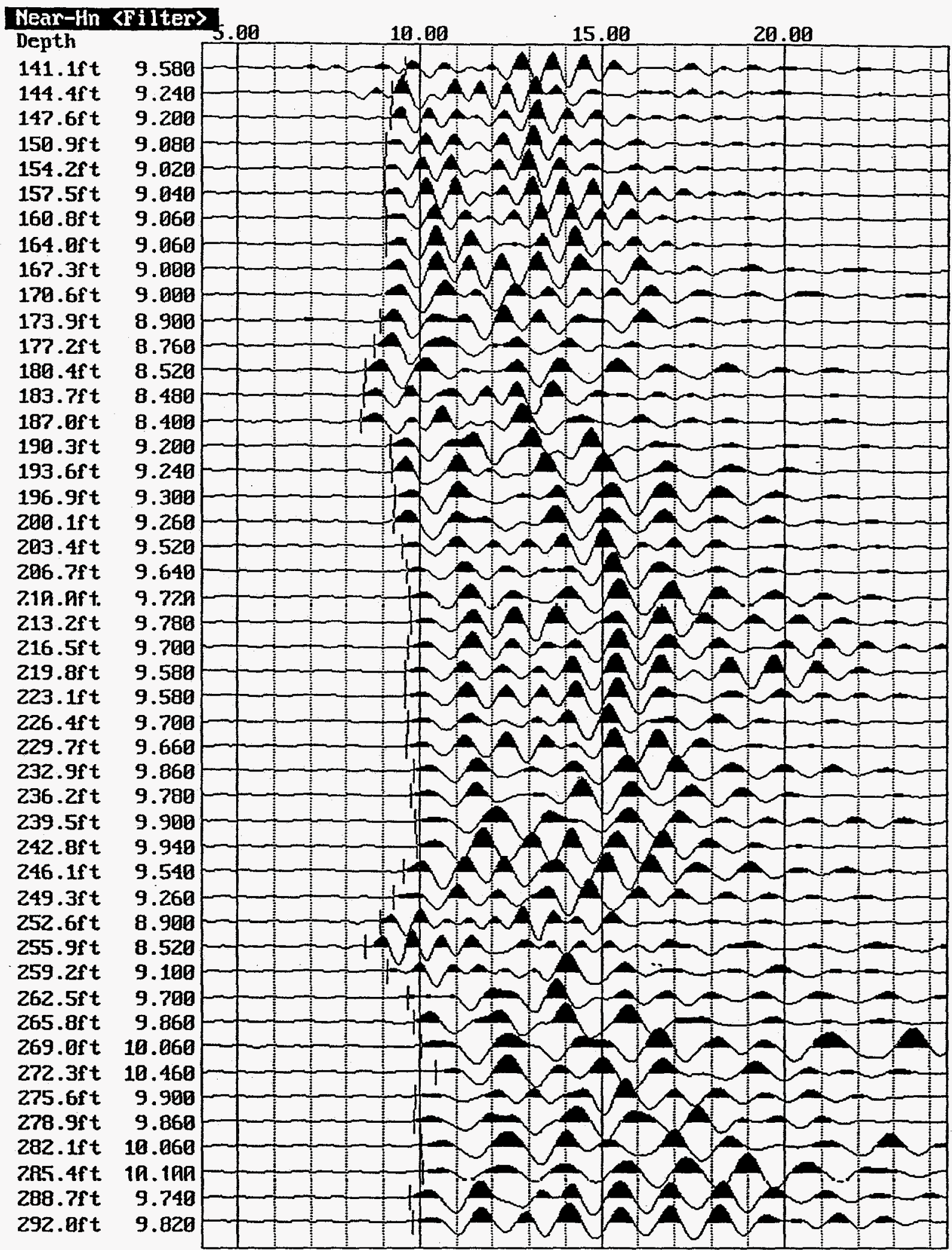

Figure B-1. Suspension $S_{H}$-wave R1 records, "Normal" direction, filtered with S - R1 first arrival picks in milliseconds . 


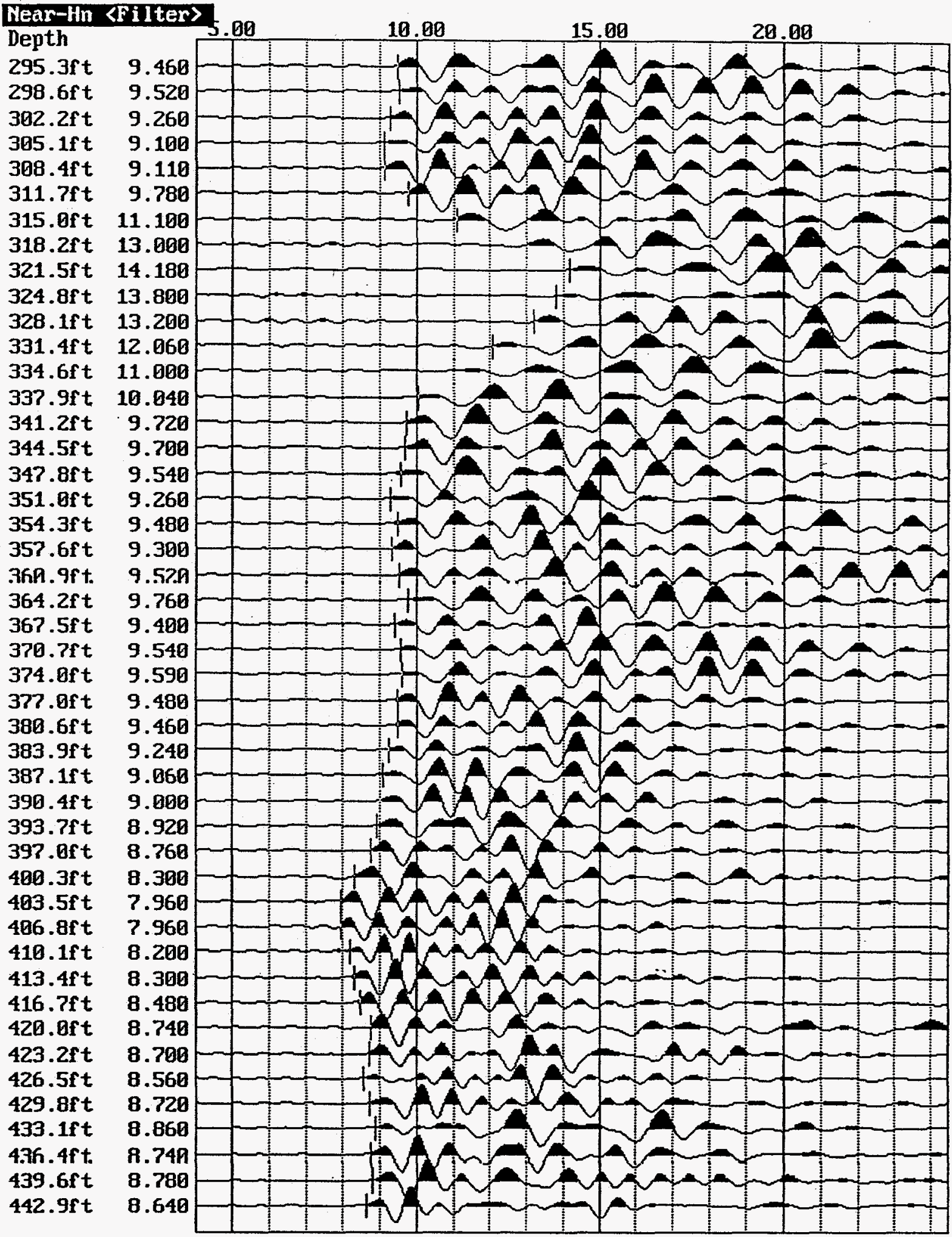

Figure B-1 continued. Suspension $S_{H^{-}}$-wave R1 records 


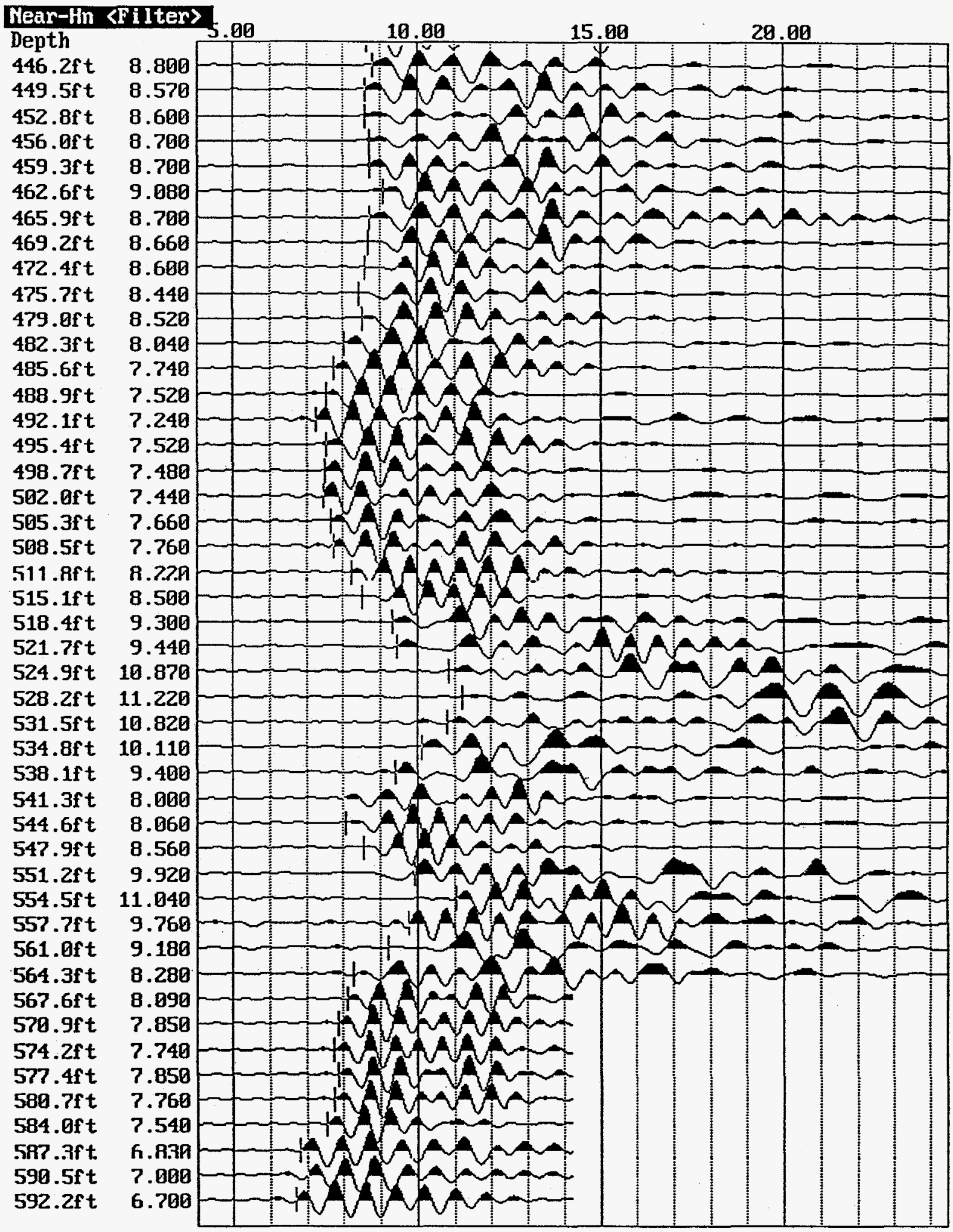

Figure B-1 continued. Suspension $\mathrm{S}_{\mathrm{H}}$-wave $\mathrm{R} 1$ records 


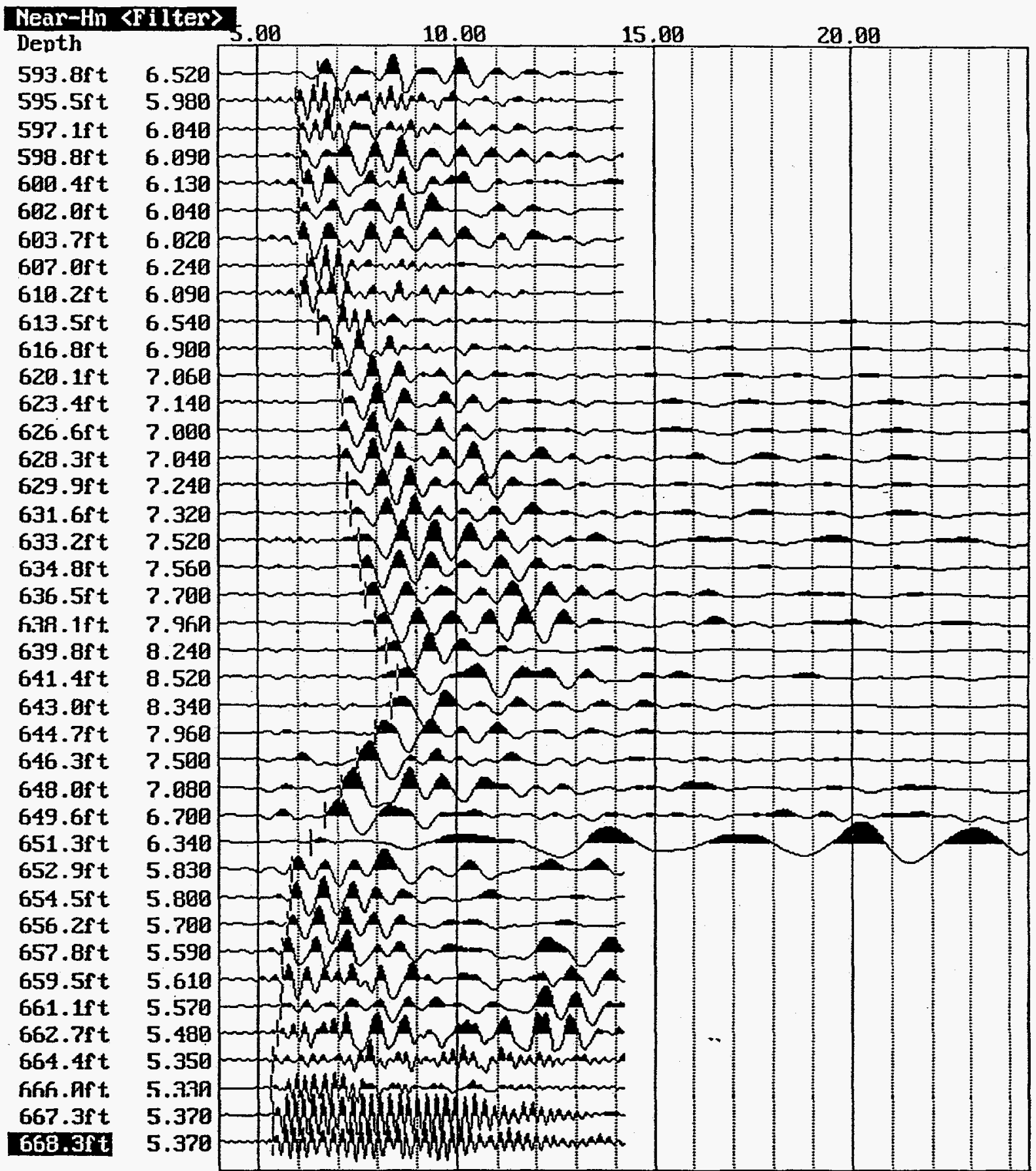

Figure B-1 continued. Suspension $\mathrm{S}_{\mathrm{H}}$-wave $\mathrm{R} 1$ records 


\section{APPENDIX C}

\section{SUSPENSION P-WAVE R1 WAVEFORM RECORDS WITH S - R1 FIRST ARRIVAL PICKS}


hear-U 〈haw

Depth

141.1ft

144.4ft

$147.6 \mathrm{ft}$

$150.9 \mathrm{ft}$

154.2ft

$157.5 f t$

160.8ft

164. Oft

167.3ft

170.6ft

$173.9 \mathrm{ft}$

$177.2 \mathrm{ft}$

$180.4 \mathrm{ft}$

$183.7 \mathrm{ft}$

187. Oft

190.3ft

$193.6 f t$

196.9ft

200.1ft

$203.4 \mathrm{ft}$

$206.7 \mathrm{ft}$

2.1ค.คft.

213.2ft

$216.5 f t$

219.8ft

223.1ft

$226.4 \mathrm{ft}$

$229.7 \mathrm{ft}$

$232.9 \mathrm{ft}$

$236.2 \mathrm{ft}$

$239.5 f t$

242.8ft

246.1ft

249.3ft

$252.6 f t$

255.9ft

259.2ft

$262.5 f t$

265.8ft

269.0ft

272.3ft

$275.6 \mathrm{ft}$

278.9ft

282.1ft

7.R5. $4 \mathrm{ft}$.

288.7ft

292. Bft
5.700

5.680

5.650

5.660

5.640

5.650

5.660

5.660

5.660

5.660

5.660

5.610

5.530

5.530

5.530

5.590

5.648

5.660

5.600

5.640

5.660

ร. КRค

5.700

5.680

5.680

5.710

5.720

5.740

5.740

5.740

5.740

5.780

5.780

5.740

5.660

5.700

5.720

5.780

5.780

5.720

5.680

5.720

5.740

5.720

5.749

5.680

5.680
6.00

8.00

10.00

12.0B

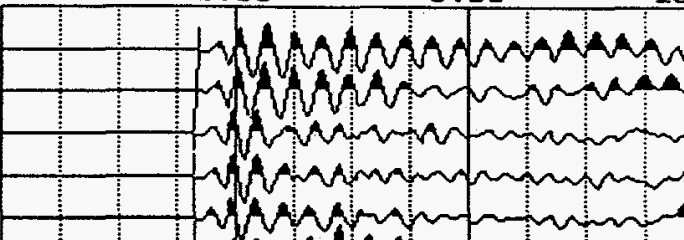

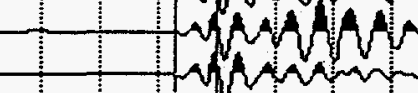

HWhan
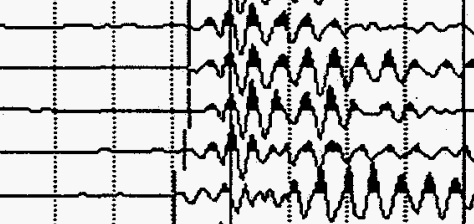

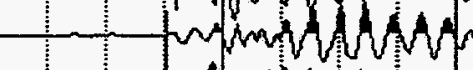
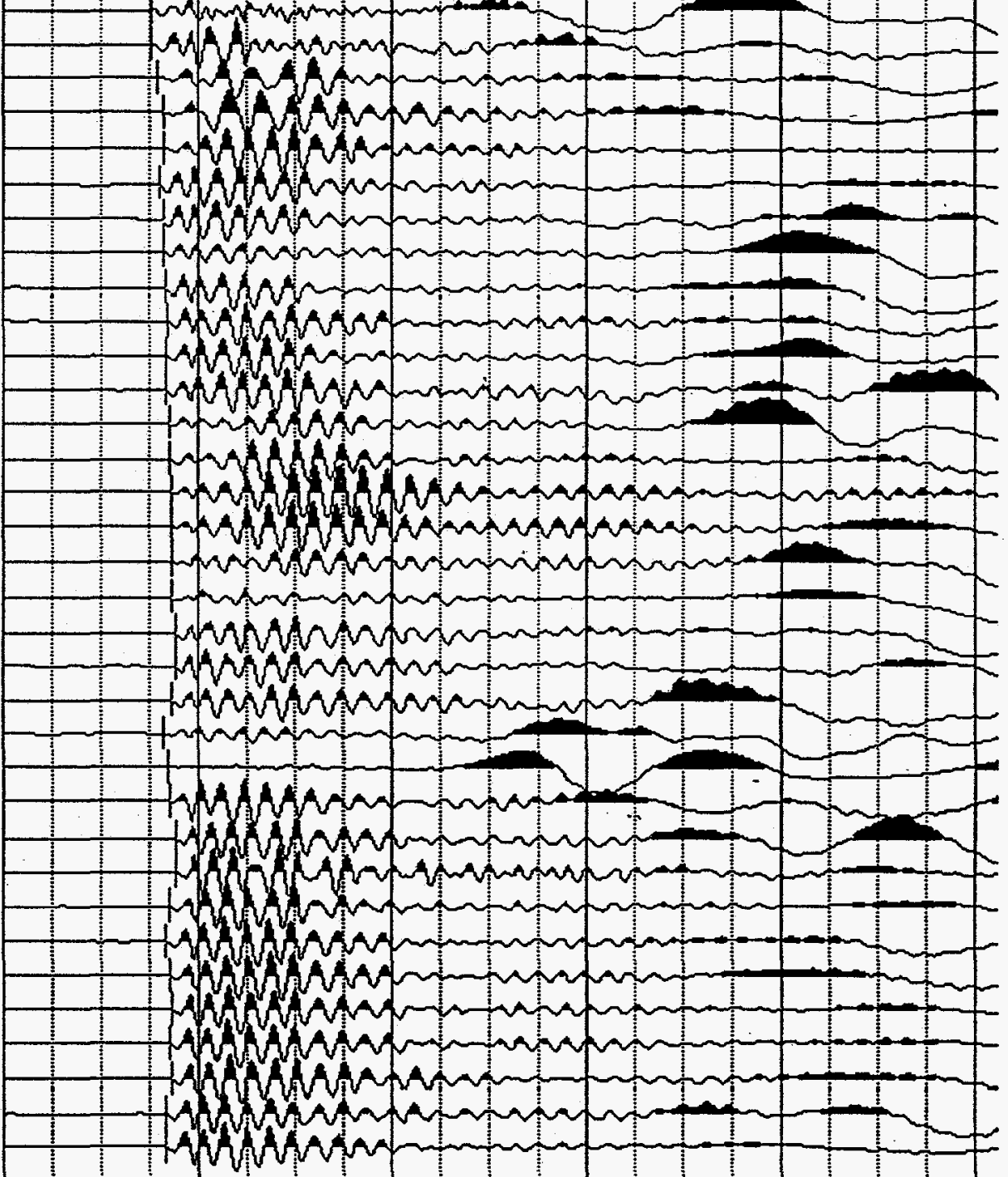

Figure $\mathrm{C}-1$. Suspension $\mathrm{S}_{\mathrm{H}}$-wave R1 records, Vertical, unfiltered with $S$ - R1 first arrival picks in milliseconds 


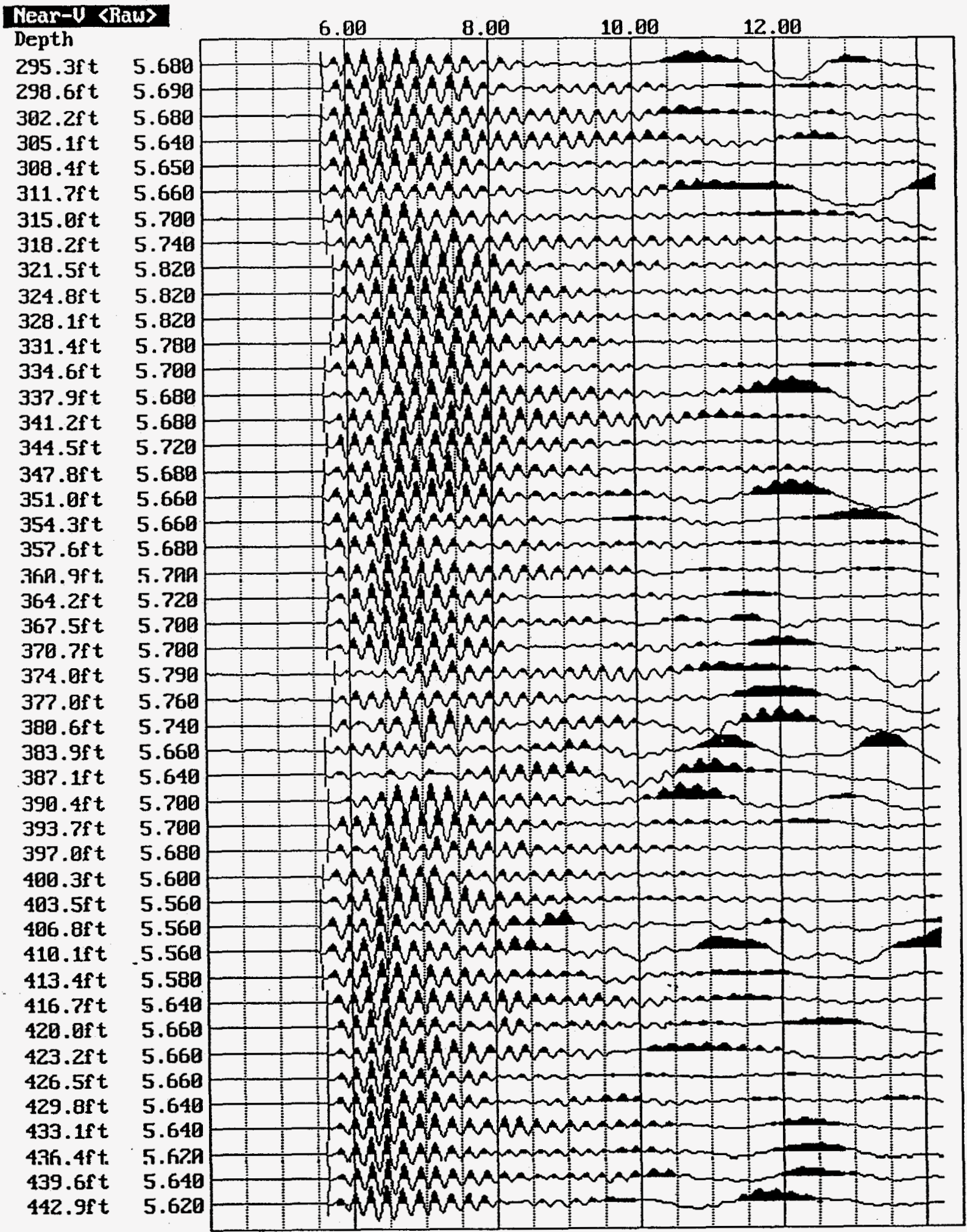

Figure $\mathrm{C}-1$ continued. Suspension $\mathrm{S}_{\mathrm{H}}$-wave $\mathrm{R} 1$ records 
Rear-U Riaus

Depth

$446.2 \mathrm{ft}$

449.5f $t$

452. Bf $t$

456. Bft

459.3ft

$462.6 f t$

465.9f $t$

469.2f t

472. $4 \mathrm{ft}$

475.7ft

479.0ft

$482.3 \mathrm{ft}$

$485.6 f t$

$488.9 f t$

492.1ft

$495.4 f t$

498.7ft

502. 0 ft

$505.3 \mathrm{ft}$

$508.5 \mathrm{ft}$

511.Rft

515.1ft

518.4ft

$521.7 f t$

$524.9 \mathrm{ft}$

$528.2 \mathrm{ft}$

$531.5 f t$

$534.8 f t$

538.1f $t$

541.3ft

$544.6 f t$

$547.9 \mathrm{ft}$

$551.2 \mathrm{ft}$

$554.5 \mathrm{ft}$

$557.7 \mathrm{ft}$

561. Oft

564.3ft

$567.6 \mathrm{ft}$

$570.9 f t$

$574.2 \mathrm{ft}$

$577.4 \mathrm{ft}$

$580.7 \mathrm{ft}$

584. Bft

$587.3 \mathrm{ft}$

$590.5 f t$

592.2ft $\begin{array}{lll}6.00 & 8.00 & 10.00\end{array}$ 12.00

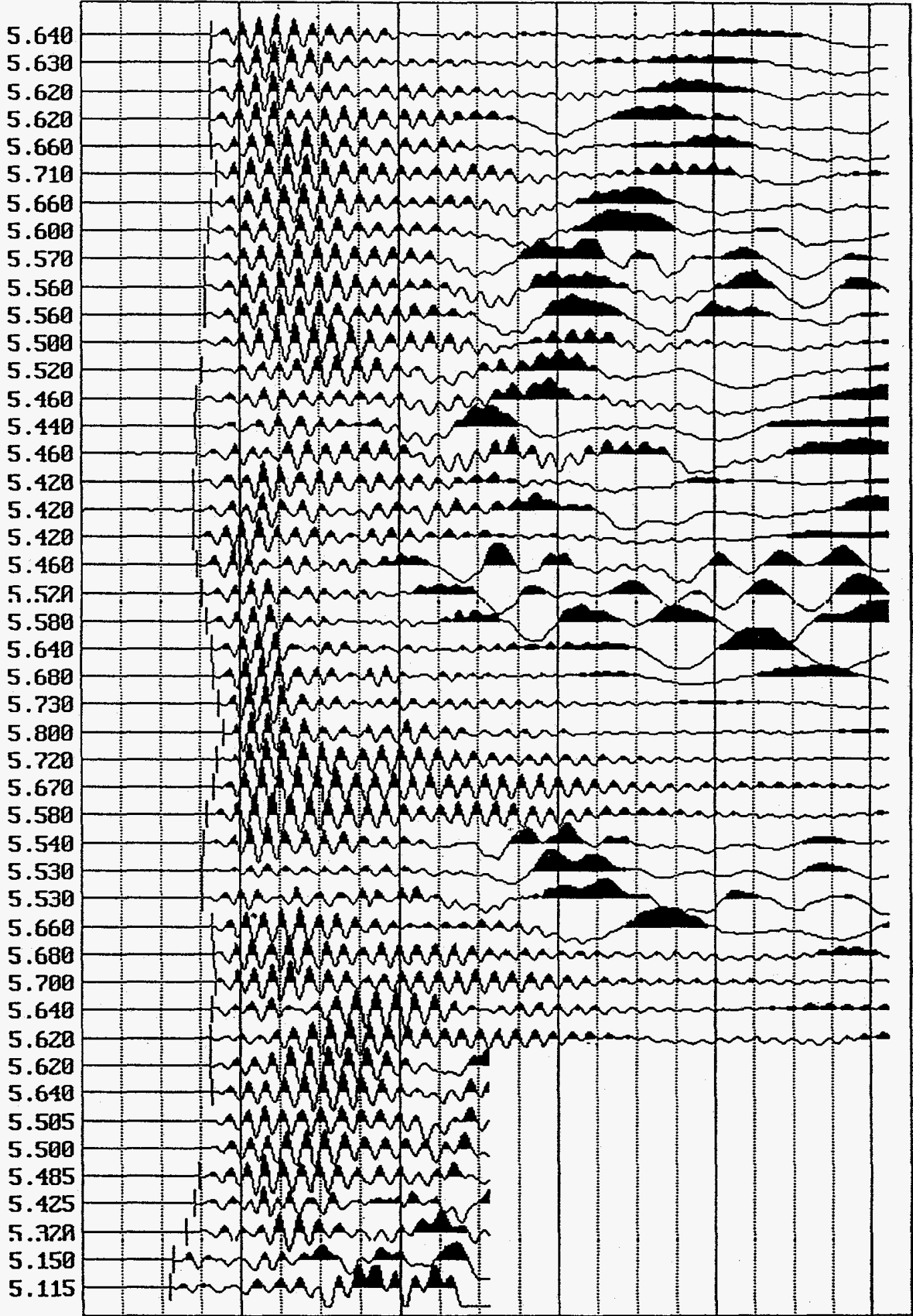

Figure $\mathrm{C}-1$ continued. Suspension $\mathrm{S}_{\mathrm{H}}$-wave $\mathrm{R} 1$ records 


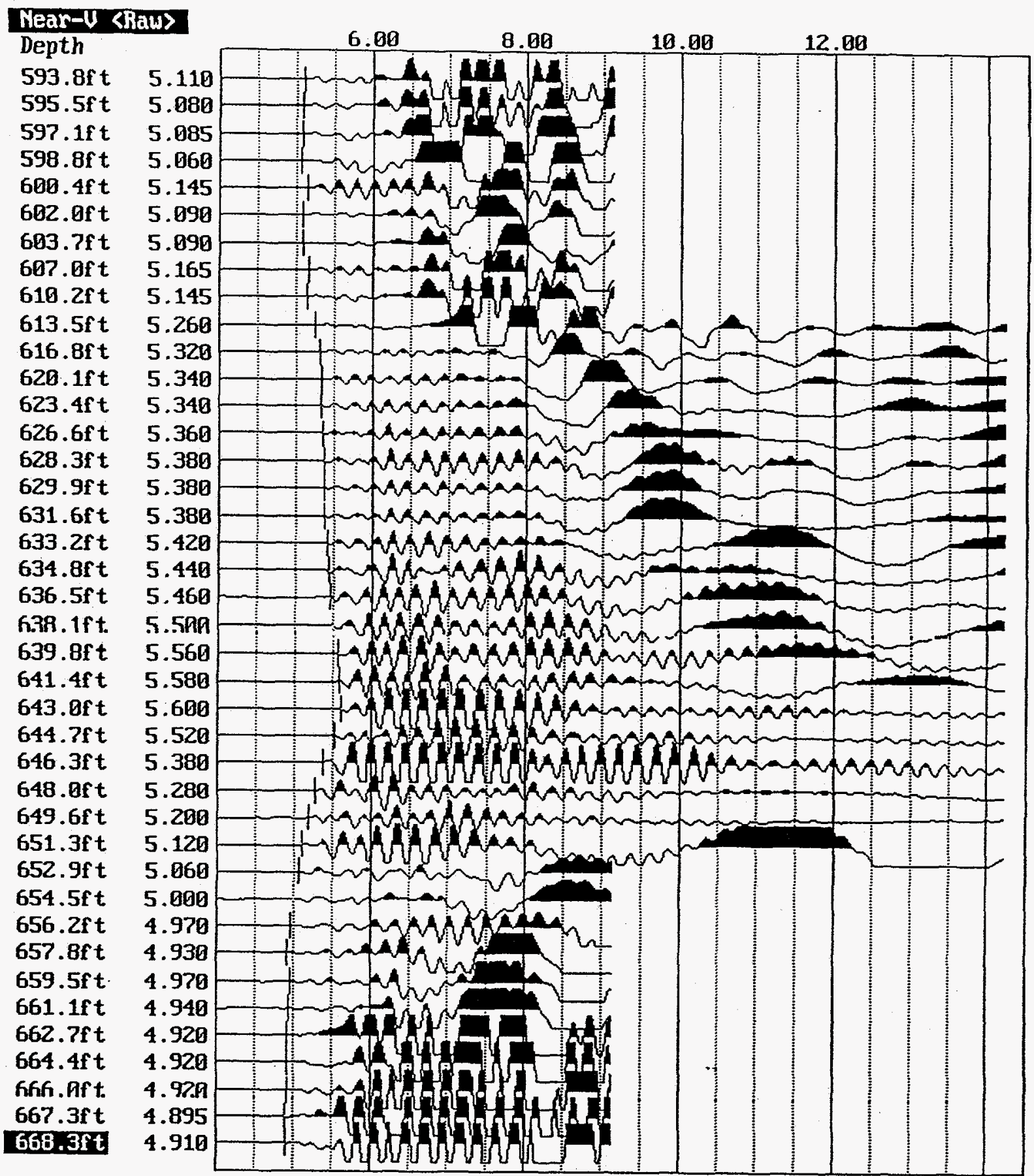

Figure $\mathrm{C}-1$ continued. Suspension $\mathrm{S}_{\mathrm{H}}$-wave $\mathrm{R} 1$ records 


\section{APPENDIX D}

\section{OYO 170 VELOCITY LOGGING SYSTEM NIST TRACEABLE}

CALIBRATION PROCEDURE 


\section{TABLE D1}

\section{AGBABIAN ASSOCIATES' VELOCITY LOGGING EQUIPMENT DESCRIPTION AND CALIBRATION PROCEDURES}

\begin{tabular}{|c|c|c|c|}
\hline EQUIPMENT & FUNCTION & $\begin{array}{c}\text { CALIBRATION } \\
\text { REQUIREMENTS }\end{array}$ & $\begin{array}{c}\text { MAINTENANCE } \\
\text { REQUIREMENTS }\end{array}$ \\
\hline $\begin{array}{c}\text { OYspension Logging } \\
\text { Data Logger }\end{array}$ & $\begin{array}{c}\text { Records data from } \\
\text { probe and sends } \\
\text { control signals to } \\
\text { probe }\end{array}$ & $\begin{array}{c}\text { Every six months, calibrate } \\
\text { sample clock using an } \\
\text { NTIS-traceable external } \\
\text { signal counter and signal } \\
\text { generator per attached } \\
\text { procedure. } \\
\text { (see Attachment D2) }\end{array}$ & $\begin{array}{c}\text { Diagnose and repair by } \\
\text { manufacturer's authorized } \\
\text { representative if sample } \\
\text { clock is out of } \\
\text { specification or } \\
\text { instrument fails. }\end{array}$ \\
$\begin{array}{c}\text { OYO Model 170 } \\
\text { Suspension Logging } \\
\text { Probe }\end{array}$ & $\begin{array}{c}\text { Suspended in } \\
\text { borehole to provide } \\
\text { both seismic source } \\
\text { and sense wave } \\
\text { arrivals at two } \\
\text { locations l meter } \\
\text { apart }\end{array}$ & $\begin{array}{c}\text { Not sensor calibration is } \\
\text { not important to the velocity } \\
\text { measurement. }\end{array}$ & $\begin{array}{c}\text { Repair as needed by } \\
\text { manufacturer-trained } \\
\text { personnel. }\end{array}$ \\
\hline $\begin{array}{c}\text { Winch System } \\
\text { (several } \\
\text { interchangeable } \\
\text { models available) }\end{array}$ & $\begin{array}{c}\text { The winch and cable } \\
\text { suspend the probe in } \\
\text { the borehole and } \\
\text { connect it to the data } \\
\text { logger }\end{array}$ & No calibration required & $\begin{array}{c}\text { Repair as needed. } \\
\text { Lubricate moving parts } \\
\text { frequently, and keep cable } \\
\text { clean. }\end{array}$ \\
\hline
\end{tabular}




\section{ATTACHMENT D2}

\section{CALIBRATION PROCEDURE FOR AGBABIAN ASSOCIATES' VELOCITY LOGGING SYSTEM}

\subsection{OYO Model 170 Data Logger Unit \\ $1.1 \quad$ Purpose}

The purpose of this calibration procedure is to verify that the sample clock of the OYO Model 170 is accurate to within $1 \%$.

\subsection{Calibration Frequency}

The calibration described in this procedure shall be performed every six months minimum.

\subsection{Test Equipment}

- Function Generator, Krohn Hite $5400 \mathrm{~B}$ or equivalent

- Frequency Counter, HP 5315A or equivalent, current NIST traceable calibration

- Test cable, function generator to OYO 170 Data Logger input channels

\subsection{Procedure}

- Connect function generator to OYO Model 170 data logger using test cable

- Set up function generator to produce a $100.0 \mathrm{~Hz}, 0.250$ volt peak square wave

- Record a data record with 100 microsecond sample period

- Measure the square wave frequency in the digital data using the data logger's screen display or utility software

\subsection{Calibration Criteria}

The measured square wave frequency in the digital data must fall between 99.0 and 101.0 $\mathrm{Hz}$ to be deemed acceptable. If outside this range, the data logger must be repaired and retested. 


\section{AgBABIAN ASSOCIATES}

engineers and consuitants

1111 South Arroyo Parkway. Sulte 470. Pasadena, CA 91105 • (818) 441-1060 • FAX: (818) 441-0757

\section{OYO 170 SUSPENSION PS LOG RECORDER CALIBRATION SHEET mamios}

SYSTEM MFG.:_OYO CORPORATION

SERIAL NO: 12004

BY: R. Steuze RH Su

COUNTER MFG.: HEWUET AREKARD

SERIAL NO: 2536 A 16357

BY:

$\operatorname{sinco}$

SIG GEN MFG: HEWLET PACKAND

SERIAL NO: $115 \perp A 12168$

BY:

simco

SYSTEM SETTINGS:

GAIN: ALL CHANNELS TO 10

FILTER: ALL LOW TO $5 \mathrm{HZ}, \mathrm{ALL}$ HI TO 25KHZ

RANGE: 10OMICROSEC

DELAY: 4MILLISEC (STD)

STACK: 1 (STD)

PULSE: 1.6MILLISEC (STD)

DISPLAY: VARIABLE (STD)

PRINT: SHORTNERT SIZE=1 (STD)

SYSTEM: MODE =SEMI AUTO (STD)

SYSTEM: DATA FORMAT =HIGH-LOW (STD)

SYSTEM: SHEAVE $=500$ PULSEM (STD)

SYSTEM: DATE $=$ CORRECT DATE \& TIME
MODEL NO:: 3331

CALIBRATION DATE: $\quad 4 / 7 / 96$

CAL DUE DATE: $10 / 2 / 96$

MODEL NO:

$53,5 A$

CALIBRATION DATE: $12 / 5 / 95$

CAL DUE DATE:

$6 / 5 / 96$

MODEL NO: $\quad 3310$ A

CALIBRATION DATE: $12 / 5 / 45$

CAL DUE DATE:

$12 / 5 / 96$

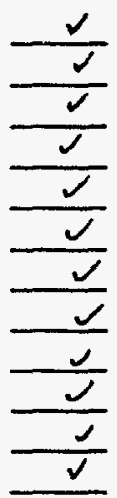

SET FREQUENCY TO $100.0 \mathrm{HZ}$, SINE WAVE AMPLITUDE TO 0.250 VOLTS PEAK $(0.1768$ VOLTS RMS). SET SQUARE WAVE AMPLITUDE TO 0.250 VOLTS PEAK. RECORD BOTH ON DISKETTE AND PAPER TAPE. ANALYZE AND PRINT WAVEFORMS FROM ANALYSIS UTILITY. ATTACH PAPER COPIES OF PRINTOUT AND PAPER TAPES TO THIS FORM. AVERAGE FREQUENCY MUST BE BETWEEN 99.0 AND 101.0 HZ.

\begin{tabular}{|l|l|l|l|l|l|l|}
\hline WAVEFORM & FILE NO & FREQ. & $\begin{array}{l}\text { TIME FOR 9 } \\
\text { CYCLES } \\
\text { Hn }\end{array}$ & $\begin{array}{l}\text { TIME FOR 9 } \\
\text { CYCLES } \\
\mathrm{Hr}\end{array}$ & $\begin{array}{l}\text { TIME FOR 9 } \\
\text { CYCLES } \\
\text { V }\end{array}$ & $\begin{array}{l}\text { AVG } \\
\text { FREQ. }\end{array}$ \\
\hline SINE & & 100.01 & 90.00 & 90.01 & 90.00 & 100.00 \\
\hline SINE & & 100.00 & 90.00 & 90.00 & 90.00 & 100.00 \\
\hline SQUARE & & 100.00 & 90.00 & 90.00 & 90.00 & 100.00 \\
\hline SQUARE & & 100.00 & 90.00 & 90.00 & 90.00 & 100.00 \\
\hline
\end{tabular}




\section{AGBABIAN ASSOCIATES}

engineers and consultants

1111 South Arroyo Parkway, Sulte 470. Pasadena, CA 91105 • (818) 441-1060 • FAX: (818) 441-0757

\section{OYO 170 SUSPENSION PS LOG RECORDER CALIBRATION SHEET im.mins}

SYSTEM MFG.:_OYO CORPORATION

SERIAL NO.: 15014

BY: 2. STEuer fuf Sh

COUNTER MFG.: HEWUETT-PACUARD

SERIAL NO.:2536A 16357

BY:

SIG GEN MFG.: HEURGT-PACKARD

SERIAL NO: 1151 A 12168

BY:

SYSTEM SETTINGS:

GAIN: ALL CHANNELS TO 10

FILTER: ALL LOW TO $5 \mathrm{HZ}$, ALL HI TO $25 \mathrm{KHZ}$

RANGE: 1OOMICROSEC

DELAY: 4MILLISEC (STD)

STACK: 1 (STD)

PULSE: 1.6MILLISEC (STD)

DISPLAY: VAAIABLE (STD)

PRINT: SHORTNERT SIZE=1 (STD)

SYSTEM: MODE=SEMI AUTO (STD)

SYSTEM: DATA FORMAT =HIGH-LOW (STD)

SYSTEM: SHEAVE $=500$ PULSEM (STD)

SYSTEM: DATE $=$ CORRECT DATE \& TIME
MODEL NO.: $\quad 3331$

CALIBRATION DATE: $4 / 2 / 96$

CAL DUE DATE: $\quad 10 / 1 / 96$

MODEL NO.: 5315 A

CALIBRATION DATE: $12 / 5 / 95$

CAL DUE DATE: $\quad 6 / 5 / 96$

MODEL NO:: $\quad 3310$ A

CALIBRATION DATE: $12 / 5 / 15$

CALDUE DATE: $\quad 12 / 5 / 96$

SET FREQUENCY TO 100.0 HZ, SINE WAVE AMPLITUDE TO 0.250 VOLTS PEAK $(0.1768$ VOLTS RMS). SET SQUARE WAVE AMPLITUDE TO 0.250 VOLTS PEAK. RECORD BOTH ON DISKETTE AND PAPER TAPE. ANALYZE AND PRINT WAVEFORMS FROM ANALYSIS UTILITY. ATTACH PAPER COPIES OF PRINTOUT AND PAPER TAPES TO THIS FORM. AVERAGE FREQUENCY MUST BE BETWEEN 99.0 AND 101.0 $\mathrm{HZ}:$

\begin{tabular}{|l|l|l|l|l|l|l|}
\hline WAVEFORM & FILE NO & FREQ. & $\begin{array}{l}\text { TIME FOR 9 } \\
\text { CYCLES } \\
\mathrm{Hn}\end{array}$ & $\begin{array}{l}\text { TIME FOR 9 } \\
\text { CYCLES } \\
\mathrm{Hr}\end{array}$ & $\begin{array}{l}\text { TIME FOR9 } \\
\text { CYCLES } \\
\text { V }\end{array}$ & $\begin{array}{l}\text { AVG } \\
\text { FREQ. }\end{array}$ \\
\hline SINE & 101 & 100.00 & 90.00 & 90.00 & 90.00 & 100.00 \\
\hline SINE & 102 & 100.00 & 90.00 & 90.00 & 90.00 & 100.00 \\
\hline SQunRe & 201 & 100.00 & 99.00 & 90.00 & 90.00 & 100.00 \\
\hline SQuPRE & 202 & 100.00 & 90.00 & 90.00 & 90.00 & 100.00 \\
\hline
\end{tabular}


Simex......

10200 PIONEER BOULEVARD, SANTA FE SPRINGS, CA 90670

CERTIFICATE OF CALIBRATION

for

KINEMETRICS

HEWLETT-PACKARD， 5315A， FREQUENCY COUNTER

I.D. NO.:5149-158 S/N:2536A16337

DEPT. NO.: TEST ASSET NO.: 0169 PO: 28508

CALIBRATION DATE: 12/05/95

NEXT CAL: 06/05/96

REASON FOR SERVICE: PERIODIC CHECK

CONDITION ON ARRIVAL: MEETS MANUFACTURER'S SPEC'S.

DISPOSITION: CLEAN/CALIBRATE TO MFR'S SPEC

TEMPERATURE: 72 Degrees Fahrenheit

RELATIVE HUMIDITY: 43 \&

STANDARDS USED:

TYPE/MODEI

FUNCTION GENERATOR/FG502

CONT-ITEM

$1004 * 713$

$1005 * 530$

$1005 * 530$

SINEWAVE GENERATOR/SG503
DUE DATE/INT ACCY .

$08 / 01 / 96$ A $\angle 5 X 10 E-10$

$10 / 10 / 96$

$10 / 10 / 96$

$05 / 16 / 96$
A FREQ+-.3PQM

A DIST<0.58

$S+/-38$ FLAT
NIST No.

FREQ WWVB

WWVB

WWVB

253135

I, JESUS PARDO, TECHNICIAN

CERTIFY THAT THE ABOVE WORK WAS COMPLETED IN ACCORDANCE WITH INDUSTRY ACCEPTED METHODS. SIMCO'S CALIBRATION SYSTEM COMPLIES WITH MIL-STD-45662A AND CALIBRATION WAS PERFORMED USING STANDARDS THAT ARE TRACEABLE TO THE NATIONAL INSTITUTE OF STANDARDS AND TECHNOLOGY.

I, PAUL KEEP, SUPERVISOR

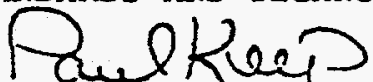

ACCEPT THIS INSTRUMENT AS CONFORMING TO CUSTOMER REQUIREMENTS.

DATED : $12 / 05 / 95$ 
SIMCO ELECTRONICS
PIONEER BOULEVARD, SANTA FE SPRINGS, CA 90670

CERTIFICATE OF CALIBRATION

for

KINEMETRICS.

HEWLETT-PACKARD, 3310A, FUNCTION GENERATOR

I.D. NO.: 5149-54 S/N:1151A12168

DEPT. NO.: TEST ASSET NO.: 0563 PO: 28508

CALIBRATION DATE: $12 / 05 / 95$

NEXT CAL: 12/05/96

REASON FOR SERVICE: PERIODIC CHECK

CONDITION ON ARRIVAL: MEETS MANUFACTURER'S SPEC'S.

DISPOSITION: CLEAN/CALIBRATE TO MFR'S SPEC

DETAIL OF WORK PERFORMED:

POWER LIGHT DOES NOT WORK.

TEMPERATURE: 72 Degrees Fahrenheit

RELATIVE HUMIDITY: $43 \%$

STANDARDS USED:

TYPE/MODEL

SCOPE/SC504

SCOPE/SC504

SCOPE/SC504

CONT-ITEM

$1004 * 194$

$1004 * 194$

$1004 * 194$

UNIVERSAL COUNTER/5335A

$1004 * 713$

DISTORTION ANALYZER/AA501 $1004 * 187$

DISTORTION ANALYZER/AA5OI $1004 * 187$

DUE DATE/INT $01 / 18 / 96 \mathrm{~S}$

$01 / 18 / 96 \mathrm{~S}$

$01 / 18 / 96$ S

$08 / 01 / 96$ A

$05 / 07 / 96$

$05 / 07 / 96 \mathrm{~S}$

ACCY.

$+/-2$ \& AMP

$+/-2 \&$ TIME

$\mathrm{RT}=<4.4 \mathrm{nS}$

$<5 X 10 \mathrm{E}-10$

$+/-1 \mathrm{~dB}$ DIST

$+/-28$ FREQ
NIST NO.

253135

WWVB

250671

FREQ WWVB

$0183 / 3393$

WWVB

I, JESUS PARDO, TECHNICIAN

CERTIFY THAT THE ABOVE WORK WAS COMPLETED IN ACCORDANCE WITH INDUSTRY ACCEPTED METHODS. SIMCO'S CALIBRATION SYSTEM COMPLIES WITH MIL-STD-45662A AND CALIBRATION WAS PERFORMED USING STANDARDS THAT ARE TRACEABLE TO THE NATIONAL INSTITUTE OF STANDARDS AND TECHNOLOGY.

I, DAUL KEEP, SUPERVISOR

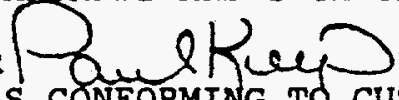
ACCEPT THIS INSTRUMENT AS CONFORMING TO CUSTOMER REQUIREMENTS. DATED : $12 / 05 / 95$

Page 1 of 1 
WSRC-RP-97-0185

Revision 0

June, 1997

Appendix C

Palynology Correspondence for GCB-1 and 2 
WSRC-RP-97-0185

Revision 0

June, 1997

This page intentionally left blank. 
Dear Doug,

I laid out the GCB2 core yesterday and selected 16 samples for paly processing. I'm not too confident that we'll recover any fossils from these samples (pretty well oxidized), but the clay between 409 and $416-6$ is a medium gray, and might, just might, yield a useable microflora. Enclosed is a copy of the transmittal letter that accompanied the samples to the processing lab, and a spreadsheet of the boxes, associated depths, and samples taken from GCB2; these are for your files.

The GCB1A sample @ 440' should be here any day, and I'm pretty excited about looking at it. I spoke with the technician who's handling the processing, and he said it looked "loaded" with fossils. When it reaches my desk and l've had a chance to examine the slides, l'll call you with my results and follow that up with a written report (again for the files).

Thanks for all you help, Doug. I'm looking forward to getting this summer's cores analyzed and to sitting down and arguing over the results.

Regards,

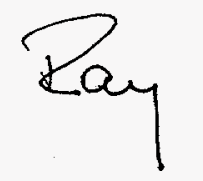




\title{
REPORT ON THE PALYNOSTRATIGRAPHY OF THE GCB2 WELL, SAVANNAH RIVER SITE
}

\author{
submitted by R. A. Christopher \\ October 8, 1996
}

\section{SUMMARY}

Twenty samples from the GCB-2 well were sampled, processed, and analyzed for palynomorphs; no age significant fossils were recovered from any of the samples, and no age determinations can be made.

Extremely rare palynomorphs and/or palynomorph fragments were observed in 5 of the samples; in addition, the organic material recovered from an additional 9 of the 20 samples was so low that only 1 kerogen slide and no biostratigraphic slides could be made for these samples. Both kerogen and biostratigraphic slides were made for the remaining 6 samples, all of which were barren of palynomorphs.

\section{BIOSTRATIGRAPHY}

The samples taken from the GCB-2 well and the corresponding results are as follows:

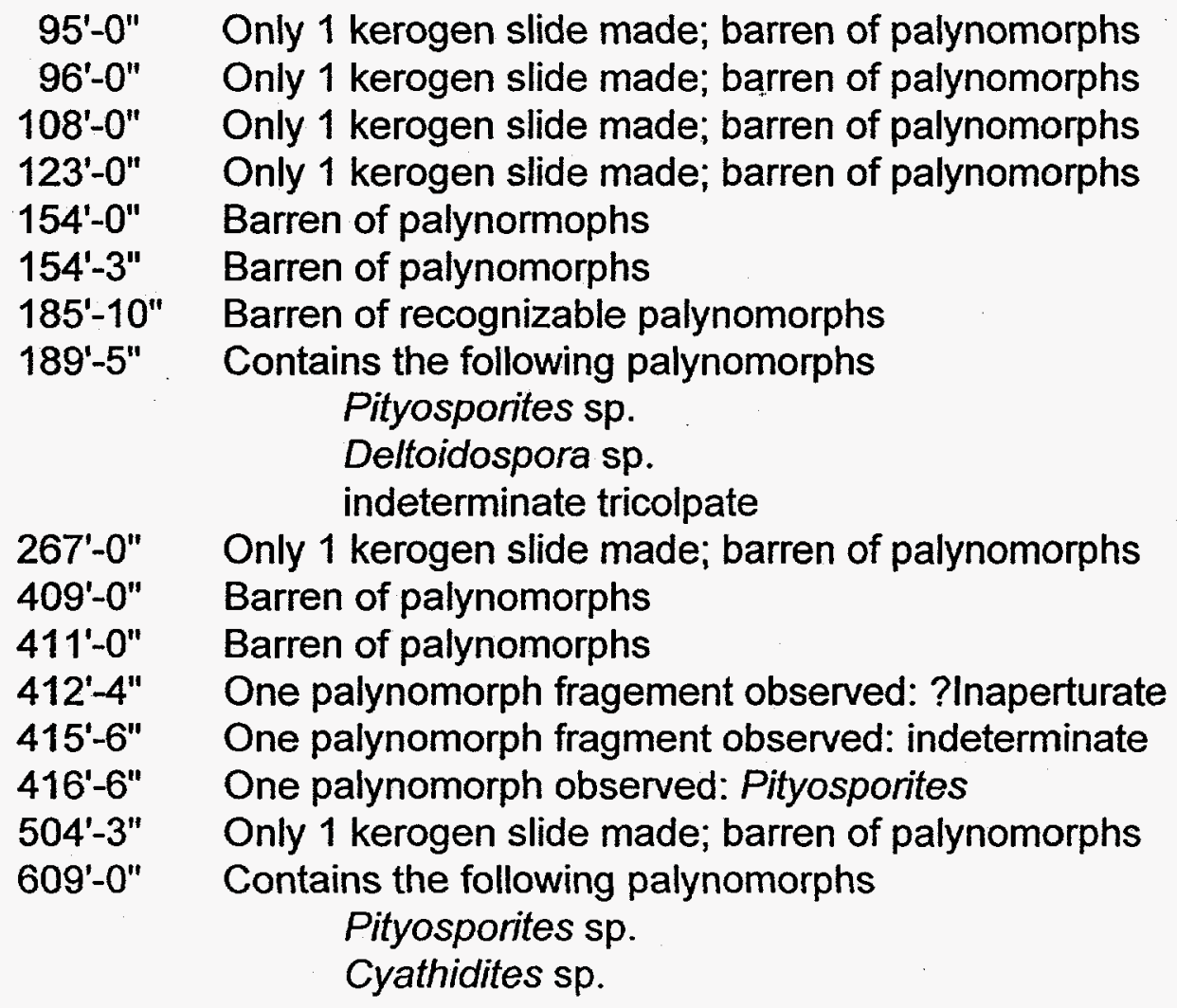


614'-2" Only 1 kerogen slide made; barren of palynomorphs 628'-3" Only 1 kerogen slide made; barren of palynomorphs 630'-6" Only 1 kerogen slide made; barren of palynomorphs 643'-0" Only 1 kerogen slide made; barren of palynomorphs

Please. call if you have any questions regarding this report.

Respectfully submitted,
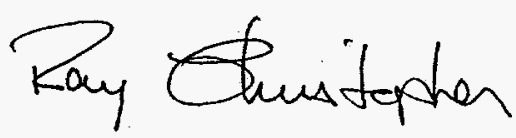

Raymond A. Christopher

cc: R. S. Van Pelt 


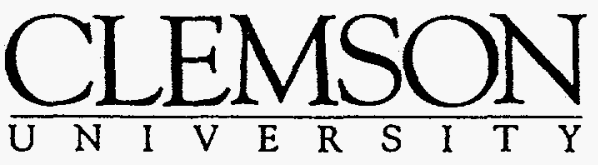

September 11, 1996

Mr. Mitch Alberts

ARCO Paleo Lab

1601 Summit Avenue

Plano, Texas 75074

Dear Mitch:

Enclosed are 16 samples from the Cretaceous section of a recently cored well located in South Carolina. Please process the samples for palynomorphs, bearing in mind that any microflora recovered will most likely contain small (10 micrometer) angiosperm pollen.

The well designation is GCB-2, and the depths at which the samples were taken are:

$\begin{array}{llll}154^{\prime}-0^{\prime \prime} & 267^{\prime}-0^{\prime \prime} & 415^{\prime}-6^{\prime \prime} & 614^{\prime}-2^{\prime \prime} \\ 154^{\prime}-3^{\prime \prime} & 409^{\prime}-0^{\prime \prime} & 416^{\prime}-6^{\prime \prime} & 628^{\prime}-3^{\prime \prime} \\ 185^{\prime}-10^{\prime \prime} & 411^{\prime}-0^{\prime \prime} & 504^{\prime}-3^{\prime \prime} & 630^{\prime}-6^{\prime \prime} \\ 189^{\prime}-5^{\prime \prime} & 412^{\prime}-4^{\prime \prime} & 609^{\prime}-0^{\prime \prime} & 643^{\prime}-0^{\prime \prime}\end{array}$

Please include the well designation and sample depth on the slide labels. For each sample, I will need two slides prepared for biostratigraphic analysis, and one slide for kerogen/maturation analysis.

There is no need to return the unprocessed sample material, but I am required to return the processed residue and prepared slides to Westinghouse Savannah River Company, so please forward the residues together with the slides when you've completed the processing.

Thanks for your help, Mitch. Please give my best to Steve, Frank, and Charles.

Regards,

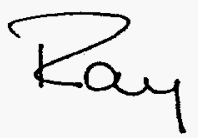

Raymond A. Christopher

enclosures

cc: R. S. Van Pelt

D. Wyatt

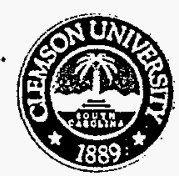

DEPARTMENT OF GEOLOGICAL SCIENCES

College of Engineering \& Science School of the Environment 340 Brackett Hall Box 341908 Clemson, SC 29634-1908 


\begin{tabular}{|c|c|c|}
\hline BOX & DEPTH RANGE & COMMENTS \\
\hline NUMBER & (ft.) & \\
\hline & 141.150 & \\
\hline $\begin{array}{l}14 \\
15\end{array}$ & $\frac{141-150}{150-160}$ & Palv sample at: 1540.15425 \\
\hline 16 & $160-170$ & \\
\hline 17 & $170-180$ & \\
\hline 18 & $180-189^{\prime}-5^{\prime \prime}$ & Paly sample at: 185.8 \\
\hline 19 & $189^{\prime}-5^{\prime \prime}-197$ & Paly sample at: 189.4 \\
\hline 20 & 197-206'-6" & \\
\hline 21 & $206^{\prime}-6^{\prime \prime}-216^{\prime}-6^{\prime \prime}$ & \\
\hline 22 & $216^{\prime}-6^{\prime \prime}-226^{\prime}-6^{\prime \prime}$ & \\
\hline 23 & $226^{\prime}-6^{\prime \prime}-236^{\prime}-6^{\prime \prime}$ & \\
\hline 24 & $236^{\prime}-6^{\prime \prime}-246^{\prime}-6^{\prime \prime}$ & \\
\hline 25 & $246^{\prime}-6^{\prime \prime}-256^{\prime}-2^{\prime \prime}$ & \\
\hline $26 \mathrm{~A}$ & $256^{\prime}-2 "-266$ & \\
\hline 26 & $266-276$ & Paly sample at: 267.0 \\
\hline 27 & $276-286$ & \\
\hline 28 & $286-296$ & \\
\hline 29 & $296-306$ & \\
\hline 30 & $306-316$ & \\
\hline 31 & $316-326$ & \\
\hline 32 & $326-336$ & \\
\hline 33 & $336-346$ & \\
\hline 34 & $346-356$ & \\
\hline 35 & $356-366$ & \\
\hline 36 & $366-376$ & \\
\hline 37 & $376-386$ & \\
\hline 38 & $386-396$ & \\
\hline 39 & $396-406$ & \\
\hline 40 & $406-416$ & Paly sample at: $409.0,411.0,412.3,415.5$ \\
\hline 41 & $416-426$ & Paly sample at: 416.5 \\
\hline 42 & $426-436$ & \\
\hline 43 & $436-446$. & \\
\hline 44 & $446-456$ & \\
\hline 45 & $456-466$ & \\
\hline 46 & $466-476$ & \\
\hline 47 & $476-486$ & \\
\hline 48 & $486-495$ & \\
\hline 49 & $495-505$ & Paly sample at: 504.25 \\
\hline 50 & $505-515$ & \\
\hline 51 & $515-525$ & \\
\hline $52^{*}$ & $525-535$ & \\
\hline $52^{*}$ & $535-545$ & \\
\hline 54 & $545-555$ & \\
\hline 55 & $555-565$ & \\
\hline 56 & $565-575$ & \\
\hline
\end{tabular}


Clemson Contact: Ray Christopher

\begin{tabular}{|l|c|l|}
\hline 57 & $575-585$ & \\
\hline 58 & $585-595$ & \\
\hline 59 & $595-605$ & \\
\hline 60 & $605-614^{\prime}-2^{\prime \prime}$ & Paly sample at: 609.0 \\
\hline 61 & $614^{\prime}-2^{\prime \prime}-624$ & Paly sample at: 614.2 \\
\hline 62 & $624-634$ & Paly sample at: $628.25,630.6$ \\
\hline 63 & $634-644$ & Paly sample at: 634.0 \\
\hline 64 & $644-654$ & Thin sections at: $644.0-644.2,645.2-645.4,650.5-650.8$ \\
\hline 65 & $654-664$ & \\
\hline 66 & $664-674$ & \\
\hline 67 & $674-683$ & \\
\hline 68 & $683-684$ & \\
\hline & & \\
\hline
\end{tabular}




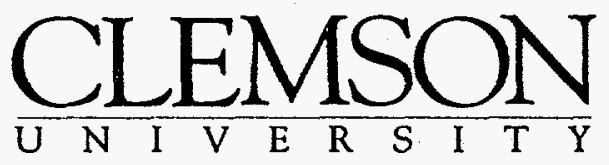

September 24, 1996

Doug Wyatt

Westinghouse Savannah River Company

Building 720-2B Room 1076

Aiken, South Carolina 29808

Dear Doug,

Enclosed is my report on the GCB1A sample $\left(439^{\prime}-6^{\prime \prime}\right)$. It's unfortunate that we have only one sample from the well, especially since this one is apparently "unique" in its stratigraphic position.

l expect the processing of the GCB2 samples to be completed in a couple of weeks, and I will let you know the schedule for the analysis and reporting of these samples when the prepared slides are returned.

Katherine informed me that you recovered a bone fragment from one of the wells, and that you're interested in identifying someone who might be able to help. There's a guy at NC State who's quite a renowned dinosaur expert, and l've been corresponding with him these past few months. I haven't been able to contact him as yet, but I'm confident that he'd be interested in looking at the sample. When l've talked with him, l'll get back to you.

Please let me know if you have any questions about the enclosed report.

Regards,

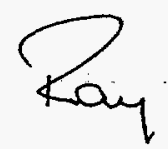

Raymond A. Christopher

enclosure

cc: $\quad$ R. S. Van Pelt

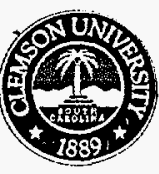

DEPARTMENT OF GEQLOGICAL SCIENCES

College of Engineering \& Science School of the Environment 340 Brackett Hall Box 341908 Clemson, SC 29634-1908 


\section{REPORT ON THE PALYNOSTRATIGRAPHY OF A SAMPLE FROM 439'-6", GCB1A WELL, SAVANNAH RIVER SITE

\author{
submitted by R. A. Christopher \\ September 23, 1996
}

\section{SUMMARY}

One sample from the GCB1A well was taken from a 4-inch thick, dark gray, laminated, slightly micaceous silty clay; the sample was processed and analyzed for palynomorphs with the following results:

- The unit is late Campanian to early Maastrichtian in age, equivalent to calcareous nannofossil zones CC23/24. As such, the unit occupies a stratigraphic position that is represented by a major unconformity in downdip wells (e.g., by an unconformity that occurs between $586 \mathrm{ft}$ and $601 \mathrm{ft}$ in the P21TA well, and between 886 and $94 \mathrm{ft}$ in the C10 well).

- The depositional environment of the unit is nonmarine, as evidenced by the abundance of nonmarine spores, pollen, plant cuticle, wood fibers, and inertinite, and by the absence of marine algae (dinoflagellates) and amorphous kerogen.

\section{BIOSTRATIGRAPHY}

\section{Age Assignment}

A late Campanian to early Maastrichtian age for the sample from 439'-6" in the GCB1A well is based on a comparison of its contained assemblage of spores and pollen with the unpublished palynological zonation developed by the writer for the Upper Cretaceous Series of the southeastern U.S. This zonation and associated ages, calcareous nannofossil zones, lithostratigraphic units as identified in the Clubhouse Crossroads \#1 core, and palynomorph markers, is illustrated in Figure 1.

The GCB1A sample at 439'-6" appears to occupy a stratigraphic position between zones $A$ and $B$ (see Figure 1). In downdip (i.e., coastal) wells, calcareous nannofossil data indicate the consistent presence of an unconformity at this horizon (Self-Trail, oral and written communication; Gohn, oral communication). Palynologically, the differences between zones $A$ and $B$ in downdip wells is readily apparent, based on the marker fossils indicated in Figure 1, and by additional criteria (i.e., other marker fossils, abundance changes, changes in the dominant pollen type or group).

The pollen assemblage from the GCB1A well, however, contains elements of both zones $A$ and $B$. Absent from the sample are the markers for both zone A (Plicatopollis cretacea, N15-31a) and zone B (?Holkopollenites sp., Osculapollis aequala). But forms that range through both zones are present in the sample (e.g., Plicapollis usitata late 
form, Baculostephanocolpites sp. A). In addition, a common to frequent element of the lower zone $A$ assemblage is an unpublished species of Momipites that is cataloged as $\mathrm{N} 15-30$. This form is smooth, both tumescent and annulate, and is easily identified even under low magnification. In the GCB1A sample, two specimens of N15-30 were observed, and in both specimens, the tunescence and annuli were weakly developed, and the surface was coarsely scabrate; these forms were interpreted as "early" forms of $\mathrm{N} 15-30$. Also present in the GCB1A sample is an unusual pollen type (undescribed pollen type, Table 1) that was also observed in the basal zone A sample in the P21TA well (at $586 \mathrm{ft})$.

\section{Depositional Environment}

The palynomoprh taxa observed in the GCB1A sample from 439'-6", and their relative abundances (based on a count of more than 350 palynomorphs), is presented in Table 1. All palynomrophs are of terrestrial origin, indicating a nonmarine depositional environment. This interpretation is supported by the presence of large fragments of plant cuticle, wood fibers, isolated bordered pits, and inertinite, which comprises approximately 50 per cent of the non-palynomorph kerogen types.

As evidenced by the data in Table 1, the assemblage is dominated by gymnosperm pollen ( $>50 \%$ of the assemblage), with the Cretaceous index fossil Rugubivesiculites spp. comprising nearly one-quarter of the entire assemblage. Angiosperm pollen comprise an additional one-quarter of the assemblage, and this microflora is diverse but lacks an overwhelmingly dominant form. Fern spores (10\%) and fungal spores (13\%) comprise the remainder of the assemblage.

The absence of marine elements, the dominance of gymnosperm pollen, the low percentage of fern spores, and the diverse angiosperm pollen suggests a terrestrial depositional environment that was well drained, and one that most likely received both air- and water-borne elements that were subject to long-distance transport.

Please contact me if you should have any questions regarding this report.

Respectfully submitted,

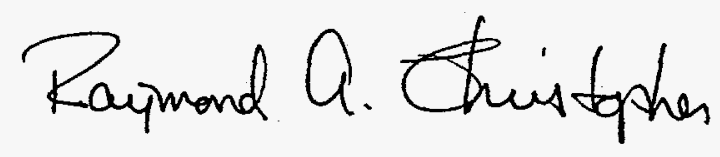

Raymond A. Christopher

Department of Geological Sciences

Clemson University

Clemson, SC 29634-1908

(864) 656-1897 
Figure 1. Palynologic zonation of the Upper Cretaceous Series of the southeastern U.S. The ages, position and duration of unconformities, and lithostratigraphic equivalents are based on calcareous nannofossil data from several downdip wells. It should be noted that each pollen zone is characterized by additional taxa and by abundance data that are not included on the figure (refer to text for a discussion of some of these additional characteristics). Alphanumeric characters in parentheses following a binomen refer either to the code(s) used by Wolfe (1976) or by the writer (unpublished). Biostratigraphic datums identified only by alphanumeric codes refer to taxa that have not been cited in the published literature. 
Figure 1.

CRETACEOUS POLLEN MARKERS, COASTAL PLAIN PROVINCE, S.E. UNITED STATES

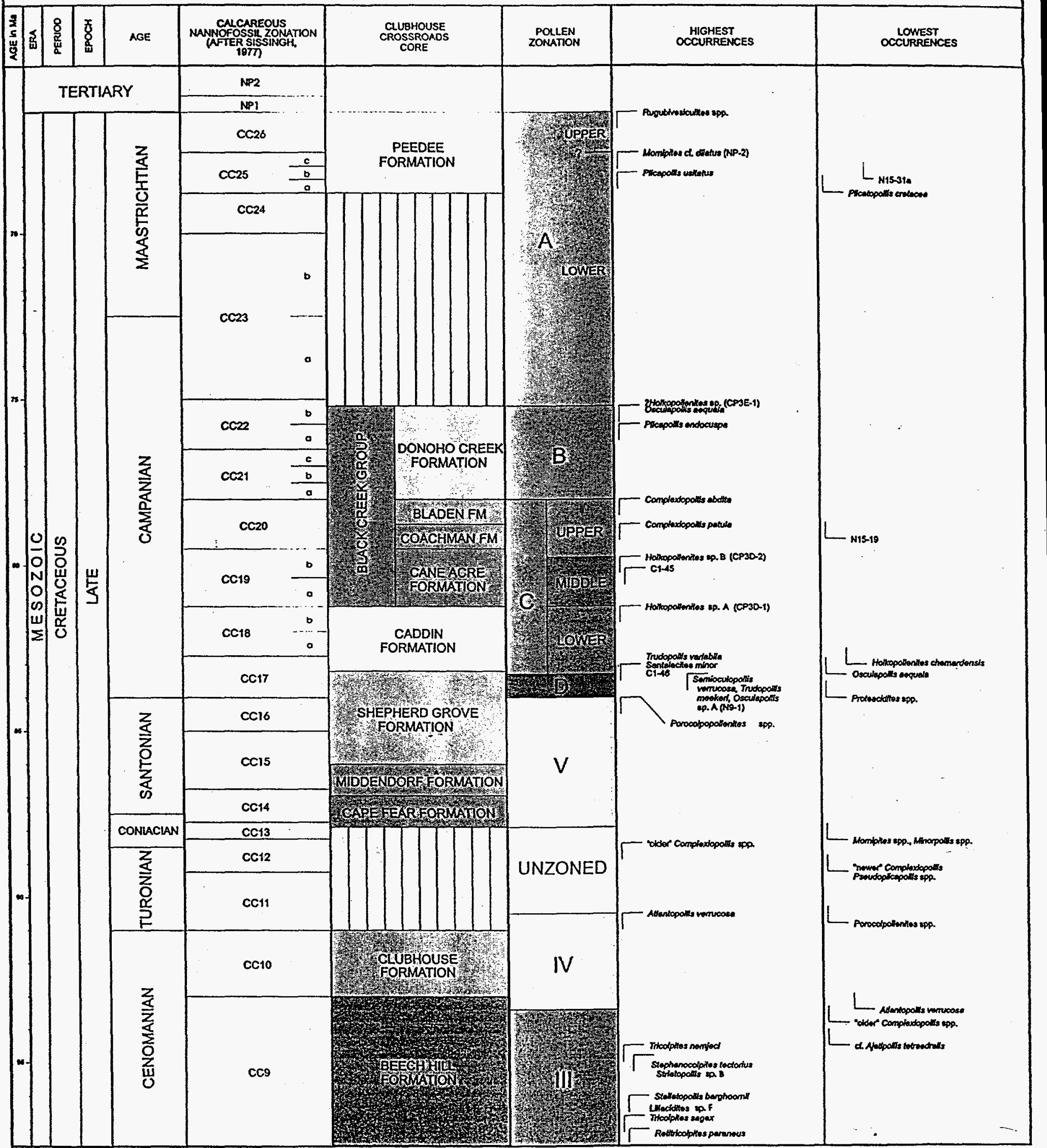


Table 1. Relative frequency of palynomorphs observed in the GCB1A sample at 439'6". Relative frequencies are presented as percentage data, based on a count of 360 palynomorphs. Those taxa whose abundance is represented by an "X" are present in the sample, but were not observed during the count of 360 grains. 
Asteropollis sp. (small form)

Undifferentiated tricolpates (C3)

Undifferentiated tricolporates (CP3)

Undescribed tricolporate form CP3-107

Nyssapollenites sp. A (CP3-20)

Holkopollenites sp. (CP3-47a)

aff. Holkopollenites sp. (CP3-85)

Undescried tricolporate form CP3-108

Holkopollenites ?chemardensis (?CP3D-3)

Brevicolporites sp. B

Ilexpollenites spp.

Libopollis jarzenii (CP3-124)

Margocolporites aff. M. cribellatus (CP3-41)

Baculostephanocolpites sp. A

Baculostephanocolpites sp. B

Nyssapollenites spp.

Minorpollis sp. (N11-2)

?Momipites sp. (N15-30)

Casuarinidites sp. (N20-2)

Pseudoplicapollis serena (NC-3)

Plicapollis usitata (NE-3 late form)

Osculapollis aff. O. perspecta (NO-4)

Proteacidites spp. (PR)

Sabalpollenites sp.

Undescribed tetracolporate form

Gymnosperm pollen

Bisaccates

Cedripites spp.

Parvisaccites spp.

Phyllocladidites spp.

Pityosporites spp.

Podocarpidites spp.

Rugubivesiculites spp.

Nonbisaccates

Araucariacites aff. A. australis

Classopollis sp.

Inaerturopollenites spp.

Sequoiaeapollenites $s p$.

Taxodiaceaepollenites hiatus

Spores

Camarozonosporites spp.

Cicatricosisporites spp.

Cyathidites major

Cyathidites minor

Deltoidospora hallii

Dictyophyllidites sp.

Laevigatosporites spp.

aff. Aratrisporites monosaccatus (M-9)

Nevesisporites sp.

Stereisporites sp.

Indeterminate affinity

Undescribed monocolpate

Perinopollenites sp.

Fungal spores

Fungal spores

$10.03 \%$

$51.67 \%$

$34.65 \%$

$0.61 \%$

$2.74 \%$

$6.38 \%$

$\mathrm{x}$

$0.30 \%$

$5.17 \%$

$0.30 \%$

$0.61 \%$

$x$

$0.30 \%$

$1.22 \%$

$x$

$3.04 \%$

$0.61 \%$

$x$

$0.91 \%$

$x$

$0.30 \%$

$x$

$x$

$x$

$1.52 \%$

$0.61 \%$

$1.52 \%$

$0.61 \%$

$6.69 \%$

$0.30 \%$

$22.80 \%$

$17.02 \%$

$17.02 \%$

$\mathrm{x}$

$7.90 \%$

$x$

$7.90 \%$

$x$

$0.30 \%$

$1.82 \%$

$x$

$x$

$2.74 \%$

$4.86 \%$

$x$

$x$

$0.30 \%$

$0.61 \%$

$x$

$0.61 \%$

$13.07 \%$

( $x=$ present, but outside the count) 


\section{REFERENCES CITED}

Wolfe, J. A., 1976, Stratigraphic distribution of some pollen types from the Campanian and lower Maestrichtian rocks (Upper Cretaceous) of the Middle Atlantic States: U.S. Geological Survey Professional Paper 977, 18p., 4 pls., 3 textfigs., 2 tables. 
WSRC-RP-97-0185

Revision 0

June, 1997

Appendix D

Core Plug VOC Data for GCB-1, 2, 3 
WSRC-RP-97-0185

Revision 0

June, 1997

This page intentionally left blank. 
MICROSEEPS

961808

.... SAVANNAH RIVER SITE .....

.... PROJECT: RELEASE 8 ....

..... SOIL CONCENTRAIONS IN (NG/G) ....

\begin{tabular}{|c|c|c|c|c|c|c|c|c|c|c|c|c|c|c|}
\hline \multirow[b]{2}{*}{ SAMPLE } & \multirow[b]{3}{*}{ BENZENE } & \multirow[b]{3}{*}{ TOLUENE } & \multirow[b]{2}{*}{ ETH.YL } & \multirow{3}{*}{$\begin{array}{r}\text { M\&P- } \\
\text { XYLENE }\end{array}$} & \multirow{3}{*}{$\begin{array}{r}\text { O- } \\
\text { XYLENE }\end{array}$} & \multirow{3}{*}{$\begin{array}{r}\text { VINYL } \\
\text { CHLORIDE }\end{array}$} & \multirow{3}{*}{$\begin{array}{l}\text { METHYLENE } \\
\text { CHLORIDE }\end{array}$} & \multirow{3}{*}{$\begin{array}{c}t-1,2- \\
\text { DCE }\end{array}$} & \multirow{3}{*}{$\begin{array}{r}\text { CHLORO } \\
\text { FORM }\end{array}$} & \multirow{3}{*}{$\begin{array}{c}1,1,1- \\
\text { TCA }\end{array}$} & CARBON & \multirow{2}{*}{$\begin{array}{r}\text { TRI } \\
\text { CHLORO }\end{array}$} & \multirow{2}{*}{$\begin{array}{l}\text { TETRA } \\
\text { CHLORO }\end{array}$} & \multirow[b]{2}{*}{ FILE } \\
\hline & & & & & & & & & & & TETRA & & & \\
\hline NAME & & & BENZENE & & & & & & & & CHLORIDE & ETHYLENE & ETHLENE & NAME \\
\hline GCB2-01 & $<2$ & 9 & $<2$ & $<2$ & $<2$ & $<100$ & $<50$ & $<1$ & $<.1$ & $<.1$ & $<.1$ & 0.4 & 0.2 & B14 224 \\
\hline GCB2.02 & $<2$ & $<2$ & $<2$ & $<2$ & $<2$ & $<100$ & $<50$ & $<1$ & 0.6 & $<.1$ & $<.1$ & 0.4 & 0.3 & 814225 \\
\hline GC82-03 & $<2$ & $<2$ & $<2$ & $<2$ & $<2$ & $<100$ & $<50$ & $<1$ & 0.6 & $<.1$ & $<.1$ & 0.3 & 0.2 & B14 226 \\
\hline GCB2-04 & $<2$ & $<2$ & $<2$ & $<2$ & $<2$ & $<100$ & $<50$ & $<1$ & 0.6 & $<.1$ & $<.1$ & 0.5 & 0.3 & B14 227 \\
\hline GCB2-05 & $<2$ & $<2$ & $<2$ & $<2$ & $<2$ & $<100$ & $<50$ & $<1$ & 0.2 & $<.1$ & $<.1$ & $<.1$ & $<.1$ & 814239 \\
\hline GCB2-06 & $<2$ & $<2$ & $<2$ & $<2$ & $<2$ & $<100$ & $<50$ & $<1$ & 0.2 & $<.1$ & $<.1$ & 0.5 & 0.4 & B14 240 \\
\hline GCB2.07 & $<2$ & $<2$ & $<2$ & $<2$ & $<2$ & $<100$ & $<50$ & $<1$ & $<.1$ & 0.2 & $<.1$ & $<.1$ & $<.1$ & B14 274 \\
\hline GCB2-08 & $<2$ & $<2$ & $<2$ & $<2$ & $<2$ & $<100$ & $<50$ & $<1$ & $<.1$ & 0.2 & $<.1$ & $<.1$ & $<.1$ & 814275 \\
\hline GCB2-09 & $<2$ & $<2$ & $<2$ & $<2$ & $<2$ & $<100$ & $<50$ & $<1$ & 0.1 & 0.2 & $<.1$ & $<.1$ & $<.1$ & B14 276 \\
\hline GCB2-10 & $<2$ & $<2$ & $<2$ & $<2$ & $<2$ & $<100$ & $<50$ & $<1$ & $<.1$ & 0.2 & $<.1$ & $<.1$ & $<.1$ & 814277 \\
\hline GCB2-10 DUP & $<2$ & $<2$ & $<2$ & $<2$ & $<2$ & $<100$ & $<50$ & $<1$ & $<.1$ & 0.2 & $<.1$ & $<.1$ & $<.1$ & B14 278 \\
\hline GCB2-11 & $<2$ & $<2$ & $<2$ & $<2$ & $<2$ & $<100$ & $<50$ & $<1$ & $<.1$ & 0.2 & $<.1$ & $<.1$ & $<.1$ & 814280 \\
\hline GCB2- 12 & $<2$ & $<2$ & $<2$ & $<2$ & $<2$ & $<100$ & $<50$ & $<1$ & $<.1$ & $<.1$ & $<.1$ & 0.4 & 0.2 & B14 241 \\
\hline GCB2- 13 & $<2$ & $<2$ & $<2$ & $<2$ & $<2$ & $<100$ & $<50$ & $<1$ & 0.1 & 0.2 & $<.1$ & $<.1$ & $<.1$ & B14 242 \\
\hline GCB2-14 & $<2$ & $<2$ & $<2$ & $<2$ & $<2$ & $<100$ & $<50$ & $<1$ & $<.1$ & 0.1 & $<.1$ & $<.1$ & $<.1$ & B14 243 \\
\hline GCB2-15 & $<2$ & $<2$ & $<2$ & $<2$ & $<2$ & $<100$ & $<50$ & $<1$ & $<.1$ & 0.2 & $<.1$ & $<.1$ & $<.1$ & B14 244 \\
\hline GCB2-16 & $<2$ & $<2$ & $<2$ & $<2$ & $<2$ & $<100$ & $<50$ & $<1$ & $<.1$ & $<.1$ & $<.1$ & $<.1$ & $<.1$ & B14 245 \\
\hline GCB2-17 & $<2$ & 10 & $<2$ & $<2$ & $<2$ & $<100$ & $<50$ & $<1$ & $<.1$ & 0.1 & $<.1$ & $<.1$ & $<.1$ & 814246 \\
\hline GCB2-18 & $<2$ & $<2$ & $<2$ & $<2$ & $<2$ & $<100$ & $<50$ & $<1$ & $<.1$ & 0.1 & $<.1$ & $<.1$ & $<.1$ & B14 247 \\
\hline GCB2-19 & $<2$ & $<2$ & $<2$ & $<2$ & $<2$ & $<100$ & $<50$ & $<1$ & 0.1 & 0.1 & $<.1$ & $<.1$ & $<.1$ & B14 248 \\
\hline GCB2-20 & $<2$ & 3 & $<2$ & $<2$ & $<2$ & $<100$ & $<50$ & $<1$ & $<.1$ & $<.1$ & $<.1$ & $<.1$ & $<.1$ & B14 249 \\
\hline GCB2-20 DUP & $<2$ & $<2$ & $<2$ & $<2$ & $<2$ & $<100$ & $<50$ & $<1$ & $<.1$ & 0.1 & $<.1$ & $<.1$ & $<.1$ & B14 250 \\
\hline $6 C 82-21$ & $<2$ & $<2$ & $<2$ & $<2$ & $<2$ & $<100$ & $<50$ & $<1$ & 0.4 & 0.2 & $<.1$ & $<.1$ & $<.1$ & 814253 \\
\hline GCB2-22 & $<2$ & $<2$ & $<2$ & $<2$ & $<2$ & $<100$ & $<50$ & $<1$ & $<.1$ & 0.1 & $<.1$ & $<.1$ & $<.1$ & B14 254 \\
\hline GCB2-23 & $<2$ & $<2$ & $<2$ & $<2$ & $<2$ & $<100$ & $<50$ & $<1$ & $<.1$ & 0.1 & $<.1$ & $<.1$ & $<.1$ & B14 255 \\
\hline GCB2-24 & $<2$ & $<2$ & $<2$ & $<2$ & $<2$ & $<100$ & $<50$ & $<1$ & $<.1$ & 0.1 & $<.1$ & $<.1$ & $<1$ & $B 14256$ \\
\hline
\end{tabular}


MICROSEEPS

961808

.... SAVANNAH RIVER SITE .....

..... PROJECT : RELEASE 8 .....

..... SOIL CONCENTRAIONS IN (NG/G) ....

\begin{tabular}{|c|c|c|c|c|c|c|c|c|c|c|c|c|c|c|}
\hline $\begin{array}{l}\text { SAMPLE } \\
\text { NAME }\end{array}$ & BENZENE & TOLUENE & $\begin{array}{r}\text { ETHYL } \\
\text { BENZENE }\end{array}$ & $\begin{array}{r}\text { M\&P- } \\
\text { XYLENE }\end{array}$ & $\begin{array}{r}0- \\
\text { XYLENE }\end{array}$ & $\begin{array}{r}\text { VINYL } \\
\text { CHLORIDE }\end{array}$ & $\begin{array}{r}\text { METHYLENE } \\
\text { CHLORIDE }\end{array}$ & $\begin{array}{c}t-1,2- \\
\text { DCE }\end{array}$ & $\begin{array}{r}\text { CHLORO } \\
\text { FORM }\end{array}$ & $\begin{array}{c}1,1,1= \\
\text { TCA }\end{array}$ & $\begin{array}{r}\text { CARBON } \\
\text { TETRA } \\
\text { CHLORIDE }\end{array}$ & $\begin{array}{r}\text { TRI } \\
\text { CHLORO } \\
\text { ETHYLENE }\end{array}$ & $\begin{array}{r}\text { TETRA } \\
\text { CHLORO } \\
\text { ETHLENE }\end{array}$ & $\begin{array}{l}\text { FILE } \\
\text { NAME }\end{array}$ \\
\hline GCB2-25 & $<2$ & $<2$ & $<2$ & $<2$ & $<2$ & $<100$ & $<50$ & $<1$ & $<.1$ & $<.1$ & $<.1$ & $<.1$ & $<.1$ & B14 257 \\
\hline $6 C 82-26$ & $<2$ & $<2$ & $<2$ & $<2$ & $<2$ & $<100$ & $<50$ & $<1$ & $<.1$ & $<.1$ & $<.1$ & $<.1$ & $<.1$ & B14 258 \\
\hline GCB2-27 & $<2$ & $<2$ & $<2$ & $<2$ & $<2$ & $<100$ & $<50$ & $<1$ & $<.1$ & $<.1$ & $<.1$ & $<.1$ & $<.1$ & B14 259 \\
\hline $\mathrm{GCB2}-28$ & $<2$ & $<2$ & $<2$ & $<2$ & $<2$ & $<100$ & $<50$ & $<1$ & $<.1$ & $<.1$ & $<.1$ & $<.1$ & $<.1$ & $B 14260$ \\
\hline GCB2-29 & $<2$ & $<2$ & $<2$ & $<2$ & $<2$ & $<100$ & $<50$ & $<1$ & $<.1$ & $<.1$ & $<.1$ & $<.1$ & $<.1$ & 814261 \\
\hline GCB2-30 & $<2$ & $<2$ & $<2$ & $<2$ & $<2$ & $<100$ & $<50$ & $<1$ & $<.1$ & $<.1$ & $<.1$ & $<.1$ & $<.1$ & $B 14262$ \\
\hline GC82-30 DUP & $<2$ & $<2$ & $<2$ & $<2$ & $<2$ & $<100$ & $<50$ & $<1$ & $<.1$ & $<.1$ & $<.1$ & $<.1$ & $<.1$ & 814263 \\
\hline GCB2-31 & $<2$ & $<2$ & $<2$ & $<2$ & $<2$ & $<100$ & $<50$ & $<1$ & 0.2 & $<.1$ & $<.1$ & $<.1$ & $<.1$ & $B 14266$ \\
\hline GCB2-32 & $<2$ & $<2$ & $<2$ & $<2$ & $<2$ & $<100$ & $<50$ & $<1$ & $<.1$ & $<.1$ & $<.1$ & $<.1$ & $<.1$ & B14 267 \\
\hline GCB2-33 & $<2$ & $<2$ & $<2$ & $<2$ & $<2$ & $<100$ & $<50$ & $<1$ & $<.1$ & $<.1$ & $<.1$ & $<.1$ & $<.1$ & B14 268 \\
\hline GCB2-34 & $<2$ & $<2$ & $<2$ & $<2$ & $<2$ & $<100$ & $<50$ & $<1$ & $<.1$ & $<.1$ & $<.1$ & $<.1$ & $<.1$ & $B 14269$ \\
\hline GCB2-35 & $<2$ & $<2$ & $<2$ & $<2$ & $<2$ & $<100$ & $<50$ & $<1$ & $<.1$ & $<.1$ & $<.1$ & 0.1 & 0.1 & B14 270 \\
\hline GC82-36 & $<2$ & $<2$ & $<2$ & $<2$ & $<2$ & $<100$ & $<50$ & $<1$ & $<.1$ & $<.1$ & $<.1$ & $<.1$ & $<.1$ & 814271 \\
\hline GCB2-37 & $<2$ & $<2$ & $<2$ & $<2$ & $<2$ & $<100$ & $<50$ & $<1$ & $<.1$ & $<.1$ & $<.1$ & $<.1$ & $<.1$ & B14 272 \\
\hline GCB2-38 & $<2$ & $<2$ & $<2$ & $<2$ & $<2$ & $<100$ & $<50$ & $<1$ & $<.1$ & $<.1$ & $<.1$ & $<.1$ & $<.1$ & B14 282 \\
\hline GCB2-39 & $<2$ & $<2$ & $<2$ & $<2$ & $<2$ & $<100$ & $<50$ & $<1$ & $<.1$ & $<.1$ & $<.1$ & $<.1$ & $<.1$ & 814283 \\
\hline GCB2-40 & $<2$ & 3 & $<2$ & $<2$ & $<2$ & $<100$ & $<50$ & $<1$ & $<.1$ & $<.1$ & $<.1$ & $<.1$ & $<.1$ & B14 284 \\
\hline GCB2-40 DUP & $<2$ & $<2$ & $<2$ & $<2$ & $<2$ & $<100$ & $<50$ & $<1$ & $<.1$ & $<.1$ & $<.1$ & $<.1$ & $<.1$ & 814285 \\
\hline $\operatorname{GCB} 2-41$ & $<2$ & $<2$ & $<2$ & $<2$ & $<2$ & $<100$ & $<50$ & $<1$ & $<.1$ & $<.1$ & $<.1$ & $<.1$ & $<.1$ & 814286 \\
\hline GCB2-42 & $<2$ & $<2$ & $<2$ & $<2$ & $<2$ & $<100$ & $<50$ & $<1$ & $<.1$ & $<.1$ & $<.1$ & $<.1$ & $<.1$ & B14 287 \\
\hline GCB2-43 & $<2$ & $<2$ & $<2$ & $<2$ & $<2$ & $<100$ & $<50$ & $<1$ & $<.1$ & $<.1$ & $<.1$ & $<.1$ & $<.1$ & B14 288 \\
\hline $6 C 82 \cdot 44$ & $<2$ & $<2$ & $<2$ & $<2$ & $<2$ & $<100$ & $<50$ & $<1$ & $<.1$ & $<.1$ & $<.1$ & $<.1$ & $<.1$ & B14 289 \\
\hline GCB3-45 & $<2$ & 35 & $<2$ & $<2$ & $<2$ & $<100$ & $<50$ & $<1$ & $<.1$ & $<.1$ & $<.1$ & $<.1$ & $<.1$ & 814348 \\
\hline GCB3-46 & $<2$ & 7 & $<2$ & $<2$ & $<2$ & $<100$ & $<50$ & $<1$ & $<.1$ & $<.1$ & $<.1$ & $<.1$ & $<.1$ & B14 349 \\
\hline $\operatorname{GCB} 3-47$ & $<2$ & $<2$ & $<2$ & $<2$ & $<2$ & $<100$ & $<50$ & $<1$ & $<.1$ & $<.1$ & $<.1$ & $<.1$ & $<.1$ & 814350 \\
\hline $6 \operatorname{ccB} 3.48$ & $<2$ & 35 & $<2$ & $<2$ & $<2$ & $<100$ & $<50$ & $<1$ & $<.1$ & $<.1$ & $<.1$ & $<.1$ & $<.1$ & B14 351 \\
\hline
\end{tabular}


MICROSEEPS

961808

.... SAVANHAH RIVER SITE .....

.... PROJECT: RELEASE 8

..... SOIL CONCENTRAIONS IN (NG/G)

\begin{tabular}{|c|c|c|c|c|c|c|c|c|c|c|c|c|c|c|}
\hline $\begin{array}{l}\text { SAMPLE } \\
\text { NAME }\end{array}$ & BENZENE & TOLUENE & $\begin{array}{r}\text { ETHYL } \\
\text { BENZENE }\end{array}$ & $\begin{array}{r}\text { M\&P- } \\
\text { XYLENE }\end{array}$ & $\begin{array}{r}0- \\
\text { XYLENE }\end{array}$ & $\begin{array}{r}\text { VINYL } \\
\text { CHLORIDE }\end{array}$ & $\begin{array}{r}\text { METHYYLENE } \\
\text { CHLOR IDEE }\end{array}$ & $\begin{array}{c}t-1,2- \\
\text { DCE }\end{array}$ & $\begin{array}{r}\text { CHLORD } \\
\text { FORM }\end{array}$ & $\begin{array}{c}1,1,1- \\
T C A\end{array}$ & $\begin{array}{r}\text { CARBON } \\
\text { TETRA } \\
\text { CHLORIDE }\end{array}$ & $\begin{array}{r}\text { TRI } \\
\text { CHLORO } \\
\text { ETHYLENE }\end{array}$ & $\begin{array}{r}\text { TETRA } \\
\text { CHLORO } \\
\text { ETHLENE }\end{array}$ & $\begin{array}{l}\text { FILE } \\
\text { NAME }\end{array}$ \\
\hline GCB3-49 & $<2$ & 7 & $<2$ & $<2$ & $<2$ & $<100$ & $<50$ & $<1$ & $<.1$ & $<.1$ & $<.1$ & $<.1$ & $<.1$ & B14 352 \\
\hline GCB3-50 & $<2$ & $<2$ & $<2$ & $<2$ & $<2$ & $<100$ & $<50$ & $<1$ & $<.1$ & $<.1$ & $<.1$ & $<.1$ & $<.1$ & $B 14353$ \\
\hline GCB3-50DUP & $<2$ & $<2$ & $<2$ & $<2$ & $<2$ & $<100$ & $<50$ & $<1$ & $<.1$ & $<.1$ & $<.1$ & $<.1$ & $<.1$ & B14 354 \\
\hline $\operatorname{GCB} 3-51$ & $<2$ & $<2$ & $<2$ & $<2$ & $<2$ & $<100$ & $<50$ & $<1$ & $<.1$ & $<.1$ & $<.1$ & $<.1$ & $<.1$ & 814358 \\
\hline GCB3-52 & $<2$ & $<2$ & $<2$ & $<2$ & $<2$ & $<100$ & $<50$ & $<1$ & $<.1$ & $<.1$ & $<.1$ & $<.1$ & $<.1$ & B14 359 \\
\hline $6 C B 3.53$ & $<2$ & $<2$ & $<2$ & $<2$ & $<2$ & $<100$ & $<50$ & $<1$ & $<.1$ & $<.1$ & $<.1$ & $<.1$ & $<.1$ & B14 360 \\
\hline GCB3-54 & $<2$ & $<2$ & $<2$ & $<2$ & $<2$ & $<100$ & $<50$ & $<1$ & $<.1$ & $<.1$ & $<.1$ & $<.1$ & $<.1$ & 814361 \\
\hline GCB3-55 & $<2$ & $<2$ & $<2$ & $<2$ & $<2$ & $<100$ & $<50$ & $<1$ & $<.1$ & $<.1$ & $<.1$ & $<.1$ & $<.1$ & B14 362 \\
\hline$G C B 3-56$ & $<2$ & $<2$ & $<2$ & $<2$ & $<2$ & $<100$ & $<50$ & $<1$ & $<.1$ & $<.1$ & $<.1$ & $<.1$ & $<.1$ & 814363 \\
\hline GCB3-57 & $<2$ & $<2$ & $<2$ & $<2$ & $<2$ & $<100$ & $<50$ & $<1$ & $<.1$ & $<.1$ & $<.1$ & $<.1$ & $<.1$ & 814364 \\
\hline $6 C B 3-58$ & $<2$ & $<2$ & $<2$ & $<2$ & $<2$ & $<100$ & $<50$ & $<1$ & $<.1$ & $<.1$ & $<.1$ & $<.1$ & $<.1$ & B14 365 \\
\hline GC83-59 & $<2$ & $<2$ & $<2$ & $<2$ & $<2$ & $<100$ & $<50$ & $<1$ & $<.1$ & $<.1$ & $<.1$ & $<.1$ & $<.1$ & 814366 \\
\hline $\operatorname{GCB} 3-60$ & $<2$ & 6 & $<2$ & $<2$ & $<2$ & $<100$ & $<50$ & $<1$ & $<.1$ & $<.1$ & $<.1$ & $<.1$ & $<.1$ & B14 377 \\
\hline GCB3-600UP & $<2$ & 6 & $<2$ & $<2$ & $<2$ & $<100$ & $<50$ & $<1$ & $<.1$ & $<.1$ & $<.1$ & $<.1$ & $<.1$ & B14 378 \\
\hline GCB3-61 & $<2$ & $<2$ & $<2$ & $<2$ & $<2$ & $<100$ & $<50$. & $<1$ & $<.1$ & $<.1$ & $<.1$ & $<.1$ & $<.1$ & $B 14380$ \\
\hline $6 \mathrm{CB3}-62$ & $<2$ & $<2$ & $<2$ & $<2$ & $<2$ & $<100$ & $<50$ & $<1$ & $<.1$ & $<.1$ & $<.1$ & $<.1$ & $<.1$ & B14 381 \\
\hline GCB3.63 & $<2$ & $<2$ & $<2$ & $<2$ & $<2$ & $<100$ & $<50$ & $<1$ & $<.1$ & $<.1$ & $<.1$ & $<.1$ & $<.1$ & 814382 \\
\hline GCB3-64 & $<2$ & 4 & $<2$ & $<2$ & $<2$ & $<100$ & $<50$ & $<1$ & $<.1$ & $<.1$ & $<.1$ & $<.1$ & $<.1$ & B14 383 \\
\hline GCB3-65 & $<2$ & 3 & $<2$ & $<2$ & $<2$ & $<100$ & $<50$ & $<1$ & $<.1$ & $<.1$ & $<.1$ & $<.1$ & $<.1$ & B14 384 \\
\hline $6 C B 3-66$ & $<2$ & $<2$ & $<2$ & $<2$ & $<2$ & $<100$ & $<50$ & $<1$ & $<.1$ & $<.1$ & $<.1$ & $<.1$ & $<.1$ & B14 394 \\
\hline $6 \operatorname{cs3}-67$ & $<2$ & $<2$ & $<2$ & $<2$ & $<2$ & $<100$ & $<50$ & $<1$ & $<.1$ & $<.1$ & $<.1$ & $<.1$ & $<.1$ & B14 395 \\
\hline GCB3-68 & $<2$ & 3 & $<2$ & $<2$ & $<2$ & $<100$ & $<50$ & $<1$ & $<.1$ & $<.1$ & $<.1$ & $<.1$ & $<.1$ & B14 396 \\
\hline GCB3-69 & $<2$ & $<2$ & $<2$ & $<2$ & $<2$ & $<100$ & $<50$ & $<1$ & $<.1$ & $<.1$ & $<.1$ & $<.1$ & $<.1$ & B14 397 \\
\hline GCB3 -70 & $<2$ & $<2$ & $<2$ & $<2$ & $<2$ & $<100$ & $<50$ & $<1$ & $<.1$ & $<.1$ & $<.1$ & $<.1$ & $<.1$ & 814398 \\
\hline GCB3-70(DUP) & $<2$ & $<2$ & $<2$ & $<2$ & $<2$ & $<100$ & $<50$ & $<1$ & $<.1$ & $<.1$ & $<.1$ & $<.1$ & $<.1$ & B14 399 \\
\hline GCB3-71 & $<2$ & $<2$ & $<2$ & $<2$ & $<2$ & $<100$ & $<50$ & $<1$ & $<.1$ & $<.1$ & $<.1$ & $<.1$ & $<.1$ & B14 400 \\
\hline
\end{tabular}


MICROSEEPS

961808

....- SAVANNAH RIVER SITE -....

PROJECT: RELEASE 8

..... SOIL CONCENTRALONS IN (NG/G) ....

\begin{tabular}{|c|c|c|c|c|c|c|c|c|c|c|c|c|c|c|}
\hline $\begin{array}{l}\text { SAMPLE } \\
\text { NAME }\end{array}$ & BENZENE & TOLUENE & $\begin{array}{r}\text { ETHYL } \\
\text { BENZENE }\end{array}$ & $\begin{array}{r}\text { M\&P- } \\
\text { XYLENE }\end{array}$ & $\begin{array}{r}0- \\
\text { XYLENE }\end{array}$ & $\begin{array}{r}\text { VINYL } \\
\text { CHLORIOE }\end{array}$ & $\begin{array}{r}\text { METHYLENE } \\
\text { CHLORIDE }\end{array}$ & $\begin{array}{c}t-1,2- \\
\text { DCE }\end{array}$ & $\begin{array}{r}\text { CHLORO } \\
\text { FORM }\end{array}$ & $\begin{array}{c}1,1,1= \\
\text { TCA }\end{array}$ & $\begin{array}{r}\text { CARBON } \\
\text { TETRA } \\
\text { CHLORIOE }\end{array}$ & $\begin{array}{r}\text { TRI } \\
\text { CHLORO } \\
\text { ETHYLENE }\end{array}$ & $\begin{array}{r}\text { TETRA } \\
\text { CHLORO } \\
\text { ETHLENE }\end{array}$ & $\begin{array}{l}\text { FILE } \\
\text { NAME }\end{array}$ \\
\hline GCB3-72 & $<2$ & 4 & $<2$ & $<2$ & $<2$ & $<100$ & $<50$ & $<1$ & $<.1$ & $<.1$ & $<.1$ & $<.1$ & $<.1$ & B14 401 \\
\hline $\mathrm{GCB} 3 \cdot 73$ & $<2$ & $<2$ & $<2$ & $<2$ & $<2$ & $<100$ & $<50$ & $<1$ & $<.1$ & $<.1$ & $<.1$ & $<.1$ & $<.1$ & 814402 \\
\hline GCB3-74 & $<2$ & 43 & $<2$ & $<2$ & $<2$ & $<100$ & $<50$ & $<1$ & $<.1$ & $<.1$ & $<.1$ & $<.1$ & $<.1$ & B14 408 \\
\hline GCB3-75 & $<2$ & $<2$ & $<2$ & $<2$ & $<2$ & $<100$ & $<50$ & $<1$ & $<.1$ & $<.1$ & $<.1$ & $<.1$ & $<.1$ & B14 409 \\
\hline GCB2-SBO1 & $<2$ & $<2$ & $<2$ & $<2$ & $<2$ & $<100$ & $<50$ & $<1$ & $<.1$ & $<.1$ & $<.1$ & $<.1$ & $<.1$ & 814223 \\
\hline GCB2-S8O2 & $<2$ & $<2$ & $<2$ & $<2$ & $<2$ & $<100$ & $<50$ & $<1$ & $<.1$ & $<.1$ & $<.1$ & $<.1$ & $<.1$ & B14 279 \\
\hline GCB2-5BO3 & $<2$ & $<2$ & $<2$ & $<2$ & $<2$ & $<100$ & $<50$ & $<1$ & $<.1$ & $<.1$ & $<.1$ & $<.1$ & $<.1$ & 814252 \\
\hline $\mathrm{GCB2}-5 \mathrm{BO} 4$ & $<2$ & $<2$ & $<2$ & $<2$ & $<2$ & $<100$ & $<50$ & $<1$ & $<.1$ & $<.1$ & $<.1$ & $<.1$ & $<.1$ & B14 265 \\
\hline GCB2-SBOS & $<2$ & $<2$ & $<2$ & $<2$ & $<2$ & $<100$ & $<50$ & $<1$ & $<.1$ & $<.1$ & $<.1$ & $<.1$ & $<.1$ & 814290 \\
\hline GCB3-5BO6 & $<2$ & $<2$ & $<2$ & $<2$ & $<2$ & $<100$ & $<50$ & $<1$ & $<.1$ & $<.1$ & $<.1$ & $<.1$ & $<.1$ & B14 357 \\
\hline GCB3-5BO7 & $<2$ & $<2$ & $<2$ & $<2$ & $<2$ & $<100$ & $<50$ & $<1$ & $<.1$ & $<.1$ & $<.1$ & $<.1$ & $<.1$ & B14 379 \\
\hline GCB3-SBO8 & $<2$ & $<2$ & $<2$ & $<2$ & $<2$ & $<100$ & $<50$ & $<1$ & 0.2 & 0.2 & $<.1$ & $<.1$ & $<.1$ & B14 393 \\
\hline
\end{tabular}




\begin{tabular}{|c|c|c|c|c|c|c|c|c|c|c|c|c|c|c|}
\hline $\begin{array}{l}\text { SAMPLE } \\
\text { NAME }\end{array}$ & BENZENE & TOLUENE & $\begin{array}{r}\text { ETHYL } \\
\text { BENZENE }\end{array}$ & $\begin{array}{c}\text { M\&P- } \\
\text { XYLENE }\end{array}$ & $\begin{array}{r}\text { O- } \\
\text { XYLENE }\end{array}$ & $\begin{array}{r}\text { VINYL } \\
\text { CHLORIDE }\end{array}$ & $\begin{array}{r}\text { METHYLENE } \\
\text { CHLORIDE }\end{array}$ & $\begin{array}{c}t-1,2- \\
\text { DCE }\end{array}$ & $\begin{array}{r}\text { CHLORO } \\
\text { FORM }\end{array}$ & $\begin{array}{c}1,1,1- \\
\text { TCA }\end{array}$ & $\begin{array}{r}\text { CARBON } \\
\text { TETRA } \\
\text { CHLORIDE }\end{array}$ & $\begin{array}{r}\text { TRI } \\
\text { CHLORO } \\
\text { ETHYLENE }\end{array}$ & $\begin{array}{r}\text { TETRA } \\
\text { CHLORO } \\
\text { ETHLENE }\end{array}$ & $\begin{array}{l}\text { FILE } \\
\text { NAME }\end{array}$ \\
\hline & & 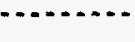 & 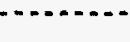 & $\cdots$ & $\cdots$ & & & 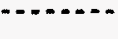 & . & 西 & 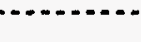 & 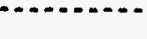 & $\cdots$ & $\cdots$ \\
\hline GC83-76 & $<2$ & $<2$ & $<2$ & $<2$ & $<2$ & $<100$ & $<50$ & $<1$ & $<.1$ & $<.1$ & $<.1$ & $<.1$ & $<.1$ & 814410 \\
\hline GCB3-77 & $<2$ & 2 & $<2$ & $<2$ & $<2$ & $<100$ & $<50$ & $<1$ & $<.1$ & $<.1$ & $<.1$ & $<.1$ & $<.1$ & B14 411 \\
\hline GCB3 -78 & $<2$ & 2 & $<2$ & $<2$ & $<2$ & $<100$ & $<50$ & $<1$ & $<.1$ & $<.1$ & $<.1$ & $<.1$ & $<.1$ & 814412 \\
\hline GCB3-79 & $<2$ & $<2$ & $<2$ & $<2$ & $<2$ & $<100$ & $<50$ & $<1$ & $<.1$ & $<.1$ & $<.1$ & $<.1$ & $<.1$ & B14 413 \\
\hline GCB3-80 & $<2$ & $<2$ & $<2$ & $<2$ & $<2$ & $<100$ & $<50$ & $<1$ & $<.1$ & $<.1$ & $<.1$ & $<.1$ & $<.1$ & 814414 \\
\hline GCB3-800UP & $<2$ & $<2$ & $<2$ & $<2$ & $<2$ & $<100$ & $<50$ & $<1$ & $<.1$ & $<.1$ & $<.1$ & $<.1$ & $<.1$ & $814 \quad 415$ \\
\hline GCB3-81 & $<2$ & $<2$ & $<2$ & $<2$ & $<2$ & $<100$ & $<50$ & $<1$ & $<.1$ & $<.1$ & $<.1$ & $<.1$ & $<.1$ & B14 419 \\
\hline $\mathrm{GCB3}-82$ & $<2$ & $<2$ & $<2$ & $<2$ & $<2$ & $<100$ & $<50$ & $<1$ & $<.1$ & $<.1$ & $<.1$ & $<.1$ & $<.1$ & 814420 \\
\hline GCB3-83 & $<2$ & 3 & $<2$ & $<2$ & $<2$ & $<100$ & $<50$ & $<1$ & $<.1$ & $<.1$ & $<.1$ & $<.1$ & $<.1$ & 814421 \\
\hline GCB3-84 & $<2$ & $<2$ & $<2$ & $<2$ & $<2$ & $<100$ & $<50$ & $<1$ & $<.1$ & $<.1$ & $<.1$ & $<.1$ & $<.1$ & B14 422 \\
\hline GCB3-85 & $<2$ & $<2$ & $<2$ & $<2$ & $<2$ & $<100$ & $<50$ & $<1$ & $<.1$ & $<.1$ & $<.1$ & $<.1$ & $<.1$ & B14 423 \\
\hline GCB3-86 & $<2$ & $<2$ & $<2$ & $<2$ & $<2$ & $<100$ & $<50$ & $<1$ & $<.1$ & $<.1$ & $<.1$ & $<.1$ & $<.1$ & B14 424 \\
\hline GCB3-87 & $<2$ & $<2$ & $<2$ & $<2$ & $<2$ & $<100$ & $<50$ & $<1$ & $<.1$ & $<.1$ & $<.1$ & $<.1$ & $<.1$ & B14 425 \\
\hline GCB3-88 & $<2$ & $<2$ & $<2$ & $<2$ & $<2$ & $<100$ & $<50$ & $<1$ & $<.1$ & $<.1$ & $<.1$ & e.1 & $<.1$ & 814426 \\
\hline GCB3-89 & $<2$ & $<2$ & $<2$ & $<2$ & $<2$ & $<100$ & $<50$ & $<1$ & $<.1$ & $<.1$ & $<.1$ & $<.1$ & $<.1$ & $814 \quad 427$ \\
\hline $6 C B 3-90$ & $<2$ & $<2$ & $<2$ & $<2$ & $<2$ & $<100$ & $<50$ & $<1$ & $<.1$ & $<.1$ & $<.1$ & $<.1$ & $<.1$ & 814428 \\
\hline GCB3-90DUP & $<2$ & $<2$ & $<2$ & $<2$ & $<2$ & $<100$ & $<50$ & $<1$ & $<.1$ & $<.1$ & $<.1$ & $<.1$ & $<.1$ & 814429 \\
\hline GCB1-91 & $<2$ & 6 & $<2$ & $<2$ & $<2$ & $<100$ & $<50$ & $<1$ & 0.8 & $<.1$ & $<.1$ & $<.1$ & $<.1$ & B14 462 \\
\hline GCB1-92 & $<2$ & 2 & $<2$ & $<2$ & $<2$ & $<100$ & $<50$ & $<1$ & 1.0 & $<.1$ & $<.1$ & $<.1$ & $<.1$ & B14 463 \\
\hline GCB1-93 & $<2$ & 5 & $<2$ & $<2$ & $<2$ & $<100$ & $<50$ & $<1$ & 0.9 & $<.1$ & $<.1$ & $<.1$ & $<.1$ & B14 464 \\
\hline GCB1-94 & $<2$ & 4 & $<2$ & $<2$ & $<2$ & $<100$ & $<50$ & $<1$ & 0.9 & $<.1$ & $<.1$ & $<.1$ & $<.1$ & B14 465 \\
\hline GCB1-95 & $<2$ & $<2$ & $<2$ & $<2$ & $<2$ & $<100$ & $<50$ & $<1$ & 0.8 & $<.1$ & $<.1$ & $<.1$ & $<.1$ & B14 466 \\
\hline GCB1-96 & $<2$ & $<2$ & $<2$ & $<2$ & $<2$ & $<100$ & $<50$ & $<1$ & 0.8 & $<.1$ & $<.1$ & 0.1 & 0.2 & 814467 \\
\hline GCB1-97 & $<2$ & 102 & $<2$ & $<2$ & $<2$ & $<100$ & $<50$ & $<1$ & 1.3 & $<.1$ & $<.1$ & 0.6 & 0.5 & B14 468 \\
\hline GCB1-98 & $<2$ & 66 & $<2$ & $<2$ & $<2$ & $<100$ & $<50$ & $<1$ & 1.2 & $<.1$ & $<.1$ & $<.1$ & $<.1$ & B14 469 \\
\hline GCB1-99 & $<2$ & 3 & $<2$ & $<2$ & $<2$ & $<100$ & $<50$ & $<1$ & 0.9 & $<.1$ & $<.1$ & 0.3 & $<.1$ & B14 470 \\
\hline GCB1-100 & $<2$ & 3 & $<2$ & $<2$ & $<2$ & $<100$ & $<50$ & $<1$ & 1.1 & $<.1$ & $<.1$ & 1.0 & 0.4 & B14 471 \\
\hline GCB $1-1000 U P$ & $<2$ & 3 & $<2$ & $<2$ & $<2$ & $<100$ & $<50$ & $<1$ & 1.1 & $<.1$ & $<.1$ & 0.8 & 0.4 & B14 472 \\
\hline GCB1-101 & $<2$ & 6 & $<2$ & $<2$ & $<2$ & $<100$ & $<50$ & $<1$ & 1.0 & $<.1$ & $<.1$ & 5.5 & 1.6 & 814476 \\
\hline GCB1-102 & $<2$ & 5 & $<2$ & $<2$ & $<2$ & $<100$ & $<50$ & $<1$ & 1.3 & $<.1$ & $<.1$ & 0.3 & $<.1$ & B14 477 \\
\hline
\end{tabular}




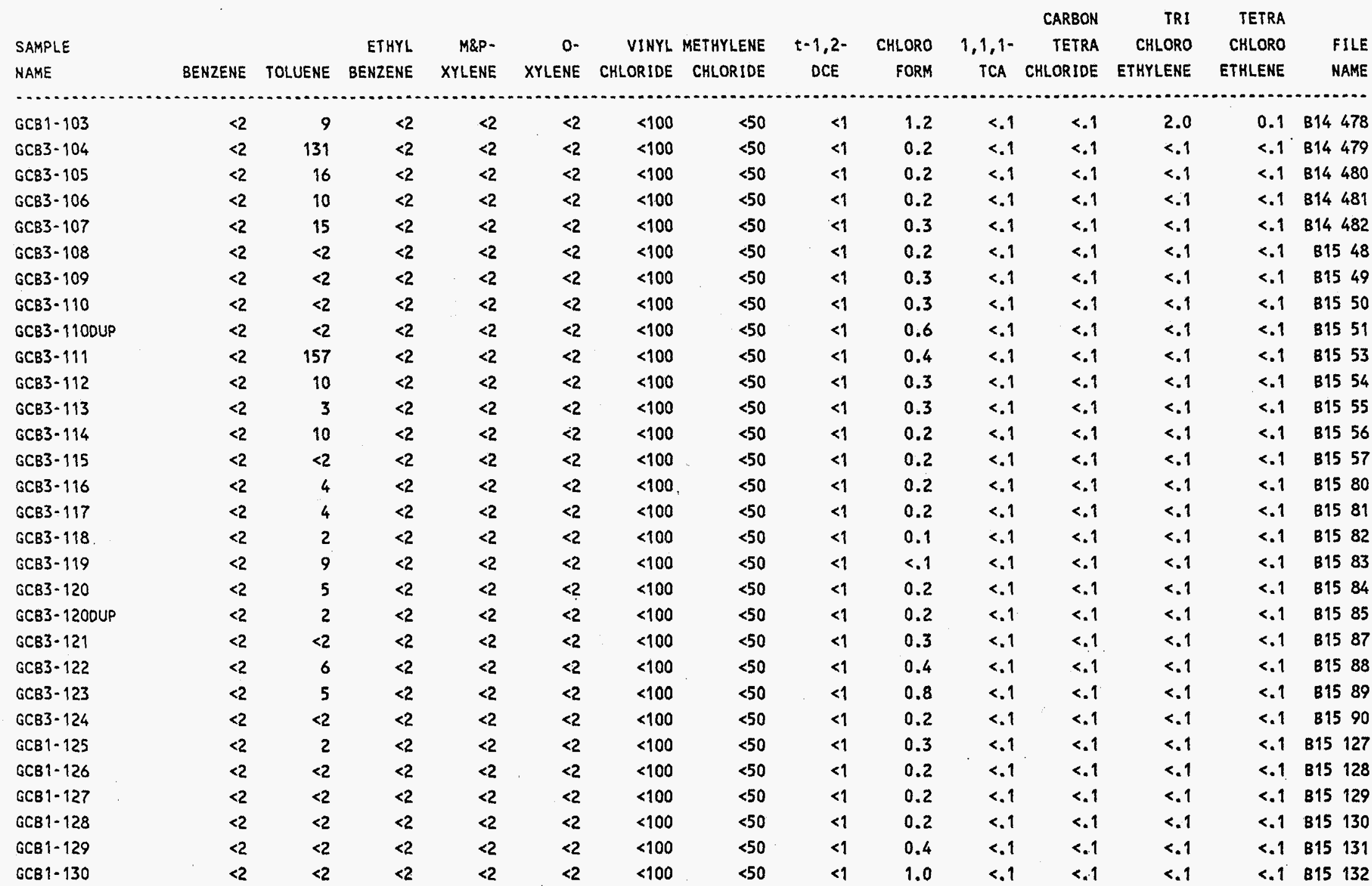




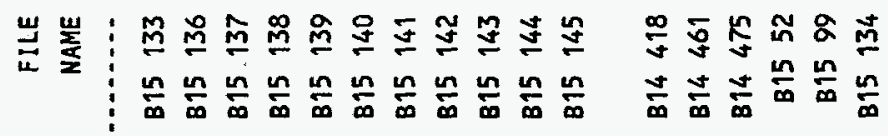

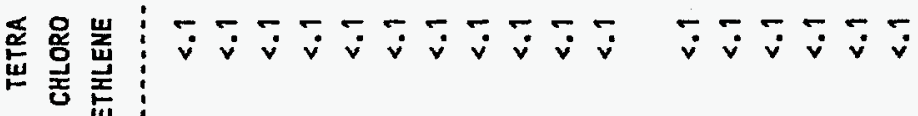

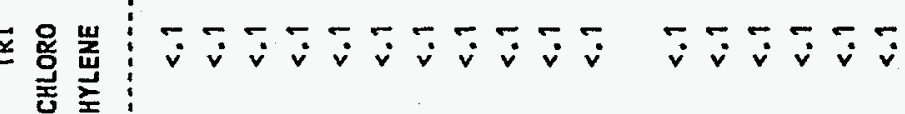

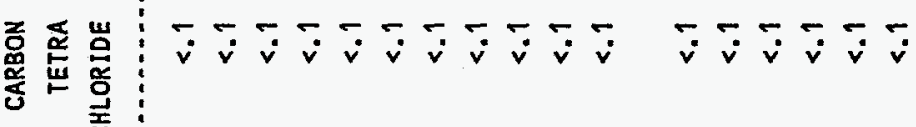

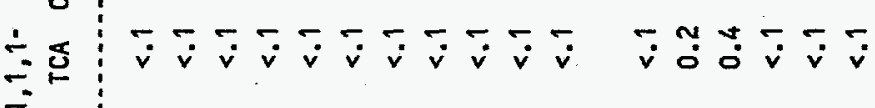

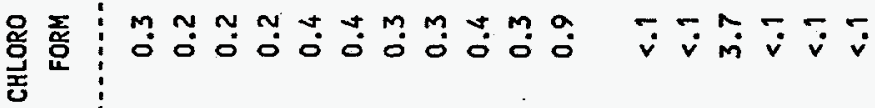

$:$\begin{tabular}{c:c}
0 \\
$\vdots$ \\
\hdashline
\end{tabular}

은 을

总芯岕

\begin{tabular}{l|lll}
$\dot{v}$ & $\bar{v} \bar{v} \bar{v} \bar{v} \bar{v} \bar{v} \bar{v} \bar{v} \bar{v} \quad \bar{v} \bar{v} \bar{v} \bar{v} \bar{v}$
\end{tabular} i

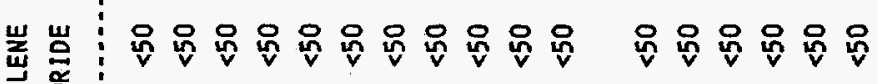
空 동

美崖

d

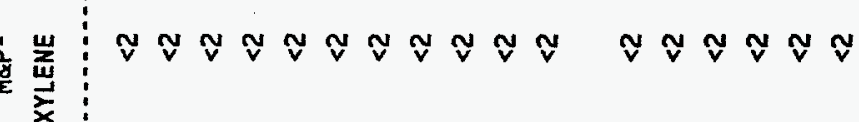

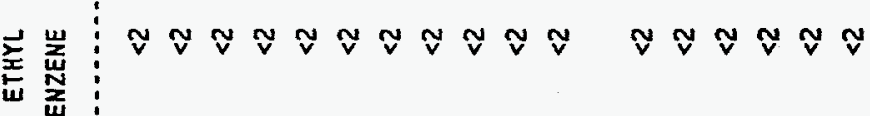

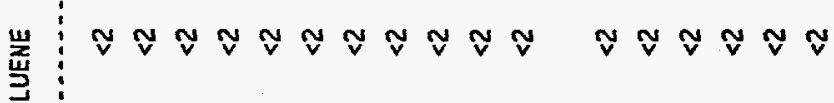

혼

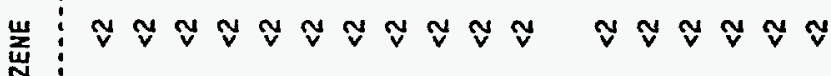




\begin{tabular}{|c|c|c|c|c|c|c|c|c|c|}
\hline $\begin{array}{l}\text { SAMPLE } \\
\text { NAME }\end{array}$ & $\begin{array}{r}\text { VINYL } \\
\text { CHLORIDE }\end{array}$ & $\begin{array}{r}\text { METHYLENE } \\
\text { CHLORIDE }\end{array}$ & $\begin{array}{c}t-1,2- \\
\text { DCE }\end{array}$ & $\begin{array}{r}\text { CHLORO } \\
\text { FORM }\end{array}$ & $\begin{array}{c}1,1,1- \\
\text { TCA }\end{array}$ & $\begin{array}{r}\text { CARBON } \\
\text { TETRA } \\
\text { CHLORIDE }\end{array}$ & $\begin{array}{r}\text { TRI } \\
\text { CHLORO } \\
\text { ETHYLENE }\end{array}$ & $\begin{array}{r}\text { TETRA } \\
\text { CHLORO } \\
\text { ETHLENE }\end{array}$ & $\begin{array}{l}\text { FILE } \\
\text { NAME }\end{array}$ \\
\hline IDUGCB2-01 & $<100$ & $<50$ & $<1$ & 2.9 & $<.1$ & $<.1$ & $<.1$ & $<.1$ & B14 191 \\
\hline IDWGCB2-02 & $<100$ & $<50$ & $<1$ & 2.9 & $<.1$ & $<.1$ & $<.1$ & $<.1$ & B14 192 \\
\hline IDWGCB2-03 & $<100$ & $<50$ & $<1$ & 1.6 & $<.1$ & $<.1$ & $<.1$ & $<.1$ & B14 220 \\
\hline IDWGCB2-04 & $<100$ & $<50$ & $<1$ & 1.6 & $<.1$ & $<.1$ & $<.1$ & $<.1$ & 814221 \\
\hline IDWGCB2-05 & $<100$ & $<50$ & $<1$ & 1.7 & $<.1$ & $<.1$ & $<.1$ & $<.1$ & B14 222 \\
\hline IDWGCB2-06 & $<100$ & $<50$ & $<1$ & 0.2 & $<.1$ & $<.1$ & $<.1$ & $<.1$ & B14 293 \\
\hline IOWGCB2-07 & $<100$ & $<50$ & $<1$ & $<.1$ & $<.1$ & $<.1$ & $<-1$ & $<.1$ & 814303 \\
\hline IDWGCB3-08 & $<100$ & $<50$ & $<1$ & 0.2 & $<.1$ & $<.1$ & $<.1$ & $<.1$ & B14 345 \\
\hline IOWGCB3-09 & $<100$ & $<50$ & $<1$ & 0.3 & $<.1$ & $<.1$ & $<.1$ & $<.1$ & 814346 \\
\hline IDWGCB3-10 & $<100$ & $<50$ & $<1$ & 0.2 & $<.1$ & $<.1$ & $<.1$ & $<.1$ & B14 347 \\
\hline IDWGCB3-11 & $<100$ & $<50$ & $<1$ & $<.1$ & $<.1$ & $<.1$ & $<.1$ & $<.1$ & 814371 \\
\hline IDWGCB3-11DUP & $<100$ & $<50$ & $<1$ & $<1$ & $<.1$ & $<.1$ & $<.1$ & $<.1$ & B14 372 \\
\hline IOWGCB3-12 & $<100$ & $<50$ & $<1$ & 0.5 & $<-1$ & $<.1$ & $<.1$ & $<.1$ & B14 389 \\
\hline IDWGCB1-13 & $<100$ & $<50$ & $<1$ & 1.3 & $<.1$ & $<.1$ & $<.1$ & $<.1$ & B14 454 \\
\hline IOWGCB1-14 & $<100$ & $<50$ & $<1$ & 1.2 & $<.1$ & $<.1$ & $<.1$ & $<.1$ & B14 455 \\
\hline IDWGCB1-15 & $<100$ & $<50$ & $<1$ & 0.9 & $<.1$ & $<.1$ & $<.1$ & $<.1$ & B14 456 \\
\hline IDWGCB1-16 & $<100^{\circ}$ & $<50$ & $<1$ & 1.3 & $<.1$ & $<.1$ & 0.1 & 0.3 & B14 457 \\
\hline IDWGCB1-17 & $<100$ & $<50$ & $<1$ & 1.1 & $<.1$ & $<.1$ & 0.3 & 0.3 & B14 458 \\
\hline IDWGCB1-18 & $<100$ & $<50$ & $<1$ & 0.4 & $<.1$ & $<.1$ & $<.1$ & $<.1$ & B15 34 \\
\hline IDWGCB1-19 & $<100$ & $<50$ & $<1$ & 0.5 & $<.1$ & $<.1$ & $<.1$ & $<.1$ & 81535 \\
\hline IOWGCB 1-20 & $<100$ & $<50$ & $<1$ & 0.6 & $<.1$ & $<.1$ & $<.1$ & $<.1$ & B15 36 \\
\hline IDWGCB1-20DUP & $<100$ & $<50$ & $<1$ & 0.5 & $<.1$ & $<.1$ & $<.1$ & $<.1$ & B15 37 \\
\hline IDWGCB1-21 & $<100$ & $<50$ & $<1$ & 0.6 & $<.1$ & $<.1$ & $<.1$ & $<.1$ & 81539 \\
\hline IDWGCB 1-22 & $<100$ & $<50$ & $<1$ & 1.2 & $<.1$ & $<.1$ & $<.1$ & $<.1$ & B15 40 \\
\hline IOWGCB1-23 & $<100$ & $<50$ & $<1$ & 1.0 & $<.1$ & $<.1$ & $<.1$ & $<.1$ & B15 41 \\
\hline IDWGCB 1-24 & $<100$ & $<50$ & $<1$ & 0.6 & $<.1$ & $<.1$ & $<.1$ & $<.1$ & B15 42 \\
\hline IDWGCB1-25 & $<100$ & $<50$ & $<1$ & 2.4 & $<.1$ & $<.1$ & $<.1$ & $<.1$ & B15 43 \\
\hline IDWGCB1-26 & $<100$ & $<50$ & $<1$ & 0.4 & $<.1$ & $-<.1$ & $<.1$ & $<.1$ & B15 62 \\
\hline 10WGCB 1-27 & $<100$ & $<50$ & $<1$ & 0.4 & $<.1$ & $<.1$ & $<.1$ & $<.1$ & 81566 \\
\hline IOWGCB1-28 & $<100$ & $<50$ & $<1$ & 0.4 & $<.1$ & $<.1$ & $<.1$ & $<.1$ & B15 67 \\
\hline IDWGCB 1-29 & $<100$ & $<50$ & $<1$ & 0.4 & $<.1$ & $<.1$ & $<.1$ & $<.1$ & B15 68 \\
\hline IDWGCB1-30 & $<100$ & $<50$ & $<1$ & 0.7 & $<.1$ & $<.1$ & $<.1$ & $<.1$ & 81569 \\
\hline IDWGCB1-30DUP & $<100$ & $<50$ & $<1$ & 0.7 & $<.1$ & $<.1$ & $<.1$ & $<.1$ & B15 70 \\
\hline IDWGCB1-31 & $<100$ & $<50$ & $<1$ & 0.5 & $<.1$ & $<.1$ & $<-1$ & $<.1$ & B15 72 \\
\hline IOWGCB1-32 & $<100$ & $<50$ & $<1$ & 0.5 & $<.1$ & $<.1$ & $<.1$ & $<.1$ & B15 73 \\
\hline IDWGCB1-33 & $<100$ & $<50$ & $<1$ & 0.5 & $<.1$ & $<.1$ & $<.1$ & $<.1$ & B15 74 \\
\hline IDWGCB1-34 & $<100$ & $<50$ & $<1$ & 0.5 & $<.1$ & $<.1$ & $<.1$ & $<.1$ & B15 75 \\
\hline IDWGCB1-35 & $<100$ & $<50$ & $<1$ & 0.5 & $<.1$ & $<.1$ & $<.1$ & $<.1$ & B15 76 \\
\hline IDWGCB1-36 & $<100$ & $<50$ & $<1$ & 0.2 & $<.1$ & $<.1$ & $<.1$ & $<.1$ & B15 105 \\
\hline IDWGCB1-37 & $<100$ & $<50$ & $<1$ & 0.2 & $<.1$ & $<.1$ & $<.1$ & $<.1$ & B15 106 \\
\hline IDWGCB 1-38 & $<100$ & $<50$ & $<1$ & 0.2 & $<.1$ & $<.1$ & $<.1$ & $<.1$ & 815107 \\
\hline IOWGCB1-39 & $<100$ & $<50$ & $<1$ & 0.2 & $<.1$ & $<.1$ & $<.1$ & $<.1$ & B15 108 \\
\hline IOWGCB1-40 & $<100$ & $<50$ & $<1$ & 0.1 & $<.1$ & $<.1$ & $<.1$ & $<.1$ & B15 109 \\
\hline IDWGCB1-40DUP & $<100$ & $<50$ & $<1$ & 0.1 & $<.1$ & $<.1$ & $<.1$ & $<.1$ & B15 110 \\
\hline IOWGCB1-41 & $<100$ & $<50$ & $<1$ & 0.2 & $<.1$ & $<.1$ & $<.1$ & $<.1$ & B15 113 \\
\hline IOWGCB1-42 & $<100$ & $<50$ & $<1$ & 0.2 & $<.1$ & $<.1$ & $<.1$ & $<.1$ & B15 114 \\
\hline IOWGCB1-43 & $<100$ & $<50$ & $<1$ & 0.2 & $<.1$ & $<.1$ & $<.1$ & $<.1$ & B15 115 \\
\hline IOWGCB 1-44 & $<100$ & $<50$ & $<1$ & 0.1 & $<.1$ & $<.1$ & $<.1$ & $<.1$ & B15 116 \\
\hline IDWGCB1-45 & $<100$ & $<50$ & $<1$ & 0.1 & $<.1$ & $<.1$ & $<.1$ & $<.1$ & B15 117 \\
\hline IDWGCB I- 46 & $<100$ & $<50$ & $<1$ & 0.2 & $<.1$ & $<.1$ & $<.1$ & $<.1$ & B15 118 \\
\hline
\end{tabular}


961807 SAVANNAH RIVER SITE

---.- PROJECT: RELEASE 7

WATER CONCENTRATIONS (UG/L)

\begin{tabular}{|c|c|c|c|c|c|c|c|c|c|}
\hline $\begin{array}{l}\text { SAMPLE } \\
\text { NAME }\end{array}$ & $\begin{array}{r}\text { VINYL } \\
\text { CHLORIDE }\end{array}$ & $\begin{array}{r}\text { METHYLENE } \\
\text { CHLORIDE }\end{array}$ & $\begin{array}{c}t-1,2- \\
D C E\end{array}$ & $\begin{array}{r}\text { CHLORO } \\
\text { FORM }\end{array}$ & $\begin{array}{c}1,1,1- \\
\text { TCA }\end{array}$ & $\begin{array}{r}\text { CARBON } \\
\text { TETRA } \\
\text { CHLORIDE }\end{array}$ & $\begin{array}{r}\text { TRI } \\
\text { CHLORO } \\
\text { ETHYLENE }\end{array}$ & $\begin{array}{r}\text { TETRA } \\
\text { CHLORO } \\
\text { ETHLENE }\end{array}$ & $\begin{array}{l}\text { FILE } \\
\text { NAME }\end{array}$ \\
\hline IDWGCB1-47 & $<100$ & $<50$ & $<1$ & 0.2 & $<.1$ & $<.1$ & $<.1$ & $<.1$ & 815119 \\
\hline IDWGCB 1-48 & $<100$ & $<50$ & $<1$ & 0.2 & $<.1$ & $<.1$ & $<.1$ & $<.1$ & B15 120 \\
\hline IDWGCB 1-49 & $<100$ & $<50$ & $<1$ & 0.2 & $<.1$ & $<.1$ & $<.1$ & $<.1$ & B15 121 \\
\hline IDWGCB 1-50 & $<100$ & $<50$ & $<1$ & 0.2 & $<.1$ & $<.1$ & $<.1$ & $<.1$ & B15 122 \\
\hline IDWGCB1-50DUP & $<100$ & $<50$ & $<1$ & 0.2 & $<.1$ & $<.1$ & $<.1$ & $<.1$ & B15 123 \\
\hline IDWGCB2-SBO1 & $<100$ & $<50$ & $<1$ & $<.1$ & $<.1$ & $<.1$ & $<.1$ & $<.1$ & B14 190 \\
\hline IDWGCB3-SBO2 & $<100$ & $<50$ & $<1$ & $<.1$ & $<.1$ & $<.1$ & $<.1$ & $<.1$ & B14 388 \\
\hline IOWGCB 1-SB03 & $<100$ & $<50$ & $<1$ & $<.1$ & $<.1$ & $<.1$ & $<.1$ & $<.1$ & B15 38 \\
\hline IDWGCB 1-SBO4 & $<100$ & $<50$ & $<1$ & $<.1$ & $<.1$ & $<.1$ & $<.1$ & $<.1$ & B15 71 \\
\hline IDWGCB1-\$B05 & $<100$ & $<50$ & $<1$ & $<.1$ & $<.1$ & $<.1$ & $<.1$ & $<.1$ & 815119 \\
\hline
\end{tabular}

